Departamento de Señales, Sistemas y Radiocomunicaciones ESCUELA TÉCNICA SUPERIOR DE INGENIEROS DE TELECOMUNICACIÓN

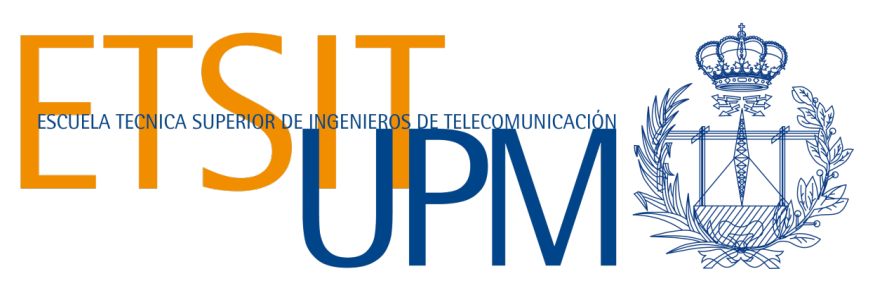

\title{
Doctoral Thesis \\ Contribution to the millimeter-wave propagation characterization for satellite and 5G wireless links
}

\author{
Author: \\ D. Domingo Pimienta del Valle \\ Telecommunication and Electronic Engineer \\ Thesis Supervisors: \\ Dr. D. José Manuel Riera Salís \\ $\mathrm{PhD}$ in Telecommunication Engineering \\ Dr. D. Pedro García del Pino \\ $\mathrm{PhD}$ in Telecommunication Engineering
}





\section{ABSTRACT}

The future spectral saturation in the SHF band in satellite systems, together with the crowding of the currently used bands and the increasing demand of high-speed data transfer in wireless communication systems have led to consider millimeter-wave frequencies (from 10 to $300 \mathrm{GHz}$ ) for allocating new applications both for satellite and terrestrial systems.

The objective of this research is to contribute in the characterization and modeling of the millimeter-wave propagation channel - specifically for satellite links in the $\mathrm{Ka}$ and $\mathrm{Q} / \mathrm{V}$ bands and in indoor wireless links at frequencies (26 and $39 \mathrm{GHz}$ ) that will be used in the future 5G systems - by collecting, processing and analyzing experimental propagation channel data. This objective is convenient since the channel propagation for these systems has not been fully characterized.

For satellite propagation analyzes, data with high availability have been used: 5 years of measurements of the KA-SAT satellite beacon in the Ka-band and 4 years of measurements of the Alphasat satellite Q-band beacon, together with ancillary meteorological information. Relevant contributions have been obtained regarding fade dynamics -fade (FD) and interfade (IFD) duration-, diversity techniques - time (TD) and orbital (OD) diversity - and the variability of statistics (rainfall rate, excess attenuation and fade dynamics), issues that have not been thoroughly treated in previous works.

Regarding FD and IFD, a modeling effort has been carried out with several function combinations. The two log-normal function is the best option to model the FD probability of occurrence and fraction of time distributions. Also, it was concluded that this function can be used for fitting the IFD probability of occurrence and inter-fading time distributions for durations shorter than $10^{6} \mathrm{~s}$ (for higher durations, a third function must be used).

The possible TD gains have been derived and compared with four models (Matricciani TD, ONERA, Greece and Joint Probability). From that, comparably good results have been obtained. The OD results were derived after a frequency scaling procedure and two models (Matricciani OD and NTUA) have been tested. It was found that in the Q-band TD gain with a delay ranging from 3 to 5 minutes may emulate the OD gain with the available angular separation $\left(18.1^{\circ}\right)$, allowing a complementary use between these techniques.

The millimeter-wave indoor experimental equipment was set up and calibrated. This and the data processing of the measurements have been treated thoroughly. Two kinds of measurements were gathered: path loss and MIMO (Multiple Input Multiple Output), both at line-of-sight (LOS) and non-line-of-sight (NLOS) conditions. Path loss measurements were taken in a corridor scenario with two sets of antennas: horns and omnidirectional. These results were fitted with several functions, and the obtained coefficients compared with models specifically developed for millimeter waves (such as the METIS, the 3GPP TR 38.901, the 5GCM, the mmMAGIC and the ITU-R Rec. P.1238-9), showing similarities in some cases. The expected waveguide-like propagation effect for LOS condition and a higher path loss in NLOS than in free space have been obtained. MIMO measurements have been gathered at 39 $\mathrm{GHz}$ with a $2 \times 2$ omnidirectional antennas setup, and the channel capacity has been tested using the condition number of the channel matrices. 



\section{RESUMEN}

La futura saturación del espectro en la banda de SHF en los sistemas satelitales y la creciente demanda de altas velocidades en la transmisión de datos en sistemas de comunicaciones inalámbricos han conducido a considerar las frecuencias milimétricas (de 10 a $300 \mathrm{GHz}$ ) para nuevas aplicaciones tanto para sistemas satelitales como para terrestres.

El objetivo de esta investigación es contribuir en la caracterización y el modelado del canal de propagación a frecuencias milimétricas - específicamente en la banda Ka y Q/V para enlaces satelitales y a frecuencias que serán utilizadas en los futuros sistemas 5G (26 y $39 \mathrm{GHz}$ ) en enlaces inalámbricos en interiores - mediante la medición, procesado y análisis de datos de canales experimentales de propagación. Este objetivo es pertinente ya que el canal de propagación para dichos sistemas no ha sido caracterizado por completo.

En el análisis de la propagación satelital se utilizan medidas con una gran disponibilidad: 5 años de la baliza del satélite KA-SAT en la banda Ka y 4 años de la baliza del satélite Alphasat en la banda Q, junto con información meteorológica adicional. Las contribuciones más relevantes se han obtenido en el modelado de la dinámica de los desvanecimientos duración de desvanecimientos (FD) y duración entre desvanecimientos (IFD) -, las técnicas de diversidad por tiempo (TD) y órbita (OD) y en la variabilidad de ciertos estadísticos, temas que no han sido tratados a profundidad en trabajos previos.

Del análisis de FD y IFD, la función que utiliza dos términos lognormales es la mejor para modelar las distribuciones de probabilidad de ocurrencia y la fracción del tiempo para FD. Esta función puede ser utilizada también para duraciones de IFD menores que $10^{6} \mathrm{~s}$ (para duraciones mayores, se necesita utilizar una tercera función).

Las posibles ganancias de TD han sido estimadas y comparadas con cuatro modelos (Matricciani TD, ONERA, Greece y Joint Probability), obteniendo buenos resultados. Los de OD fueron derivados luego de un escalado en frecuencia, además se evaluaron dos modelos (Matricciani OD y NTUA). Se obtuvo que la ganancia de TD en la banda Q con un retardo de 3 a 5 minutos puede emular la ganancia que se obtendría con OD con la separación angular dada $\left(18.1^{\circ}\right)$, permitiendo una utilización complementaria entre estas técnicas.

Se han tratado a profundidad el montaje y calibrado del equipamiento experimental utilizado en las medidas de ondas milimétricas en interiores y su procesado. Se tomaron dos tipos de medidas en interiores: medidas de pérdidas de propagación y MIMO, ambas en condiciones LOS y NLOS. Las medidas de pérdidas fueron realizadas en un pasillo con dos configuraciones de antenas: bocinas y omnidireccional. Los resultados fueron ajustados con varias funciones, comparando los coeficientes obtenidos con modelos desarrollados específicamente para estas frecuencias (METIS, 3GPP TR 38.901, 5GCM, mmMAGIC y ITU-R Rec. P.1238-9), observando similitudes en algunos casos. Los resultados muestran el esperado efecto de propagación de guiado en condiciones LOS y unas pérdidas mayores en NLOS que en el espacio libre. Las medidas MIMO fueron obtenidas a $39 \mathrm{GHz}$ utilizando una configuración $2 \times 2$ de antenas omnidireccionales, y la capacidad del canal ha sido evaluada mediante el número de condición de las matrices de canal. 



\section{AGRADECIMIENTOS}

Quisiera mostrar mi agradecimiento a todas las personas que han contribuido en la culminación de este trabajo. En primer lugar a mi familia, por su apoyo y aliento en cada etapa de mi vida, lo que me ha llevado hasta aquí. He pasado tres años dedicado a esta tarea, lejos de casa, pero sintiendo su apoyo y orgullo en cada momento. También dedico este trabajo a mi hijo, el cual ha tenido que compartir mi dedicación en estos sus primeros años de vida, y a su mamá y mi compañera, que ha tenido que asumir muchos de mis deberes familiares, por lo que estaré eternamente agradecido.

Agradezco profundamente a mis tutores Dr. D. José Manuel Riera Salís y Dr. D. Pedro del Pino García su apoyo y guía en un tema tan interesante de investigación como la radiopropagación. Su ayuda durante estos años, sus profundas revisiones al documento final y los debates sostenidos han servido para mejor el trabajo que se presenta. Espero no haber defraudado sus expectativas.

También quisiera mostrar mi sincera gratitud a Dr. D. José Manuel Riera Salís, quien me presentó la oportunidad de cumplir mi sueño y convertirme en Doctor, y a Dr. D. José Raúl Vento Álvarez, quien me enseñó el camino a seguir con ese fin.

Quisiera reconocer el trabajo y las contribuciones previas realizadas por Dr. D. Pedro García del Pino y Dr. D. Gustavo Siles Soria en los experimentos satelitales. Sin todas las tareas que acometieron, no se hubiera logrado la calidad de los datos y análisis aquí presentados.

Una parte importante de los resultados obtenidos en la campaña de propagación en interiores ha sido posible gracias a la colaboración de Dr. D. Luis Mendo Tomás, quien ha programado varios de los códigos del procesado de los datos; y a D. Pedro Saíz Coronado y D. Sergio Hernández Sáenz, quienes han participado en la campaña de medición y en el postprocesado de los datos. Mi más sincero agradecimiento por sus contribuciones.

Agradecer a todos los miembros del GTIC por su apoyo y haberme acogido fraternalmente el Grupo. En este me he enriquecido en cada debate durante nuestros almuerzos, las horas de laboratorio y los viajes que hemos compartido.

Por último, pero no menos importante, agradezco a aquella instituciones que me han permitido completar los resultados que se presentan: a ONERA la posibilidad de emplear su software de diversidad en tiempo; al Ministerio de Economía y Competitividad por la asignación de la Ayuda Pre-doctoral que me ha financiado este tiempo y a la Universidad Politécnica de Madrid, especialmente el Departamento de Señales, Sistemas y Radiocomunicaciones, por permitirme participar en su Programa de Doctorado. 



\section{GLOSSARY}

3GPP

$5 \mathrm{G}$

5 GCHFB

5GPPP

ABG

ABGX

$\mathrm{ACM}$

ACTS

ADC

amsl

ARQ

BUPT

$\mathrm{C} / \mathrm{N}_{0}$

$\mathrm{CCDF}$

CDF

CEA-Leti

CI

CIF

CIFX

CIX

CldMod

CMCC

COST

CRC

DBSG3

D-H

DLPC

DoA

DoD

DSD

ECMWF 3rd Generation Partnership Project

Fifth Generation of mobile service

5G/5G+ Communications in Higher Frequency Bands

5G infrastructure Public Private Partnership

Alpha-Beta-Gamma model

Alpha-Beta-Gamma model with a polarization factor

Adaptive Coding and Modulation

Advanced Communications Technology Satellite

Analog to Digital Converter

Above the mean sea level

Automatic repeat request

Beijing University of Posts and Telecommunications

Carrier to Noise spectral density

Complementary Cumulative Distribution Function

Cumulative Distribution Function

Commissariat à l'Energie Atomique et aux Energies AlternativesLaboratoire d'électronique des technologies de l'information (in French)

Close-In free space reference distance model

Close-In free space reference distance model with Frequency dependent path loss exponent

Close-In free space reference distance model with Frequency and Polarization dependent path loss exponent

Close-In reference distance model with XPD

Cloud Model

China Mobile Communications Corporation

European Cooperation on Science and Technology

Communication Research Centre Canada

Databank of Study Group 3

Dissanayanke-Haidara Model

Down-Link Power Control

Direction of Arrival

Direction of Departure

Drop Size Distribution

European Center for Medium Range Weather Forecast 
EIRP Effective Isotropic Radiated Power

ERA

ECMWF Re-Analysis

ESA

European Space Agency

ETRI

Electronics and Telecommunications Research Institute

ETSI

Escuela Técnica Superior de Ingenieros (in Spanish)

EU

European Union

EUTELSAT

European Telecommunications Satellite Organization

EXCELL

Exponential Cell Model

$\operatorname{Exp}+\operatorname{Exp}+\operatorname{Exp}$

Three exponential function combination

$\operatorname{Exp}+\log$

Exponential and Log-normal distribution combination

FD

Fade Duration

FEC

Forward Error Correction

FFT

Fast Fourier Transform

FI

Floating-Intercept

FS

Free Space

FSPL

Free Space Path Loss

FSS

Fixed Satellite Service

$\mathrm{G} / \mathrm{T}$

Gain/noise Temperature

GNSS

Global Navigation Satellite System

GPCC

Global Prediction Climatology Centre

GSCM

Geometry-based Stochastic Channel Model

GTIC

Information and Communication Technologies Research Group (Grupo

de Tecnologías de la Información y las Comunicaciones, in Spanish)

HYCELL

Hybrid Cell Model

IDU

Indoor Unit

IEEE

Institute of Electrical and Electronics Engineers

IF

Intermediate Frequency

IFD

Inter-Fade Duration

IGS

International GNSS Service

IMDEA

Instituto Madrileño De Estudios Avanzados (in Spanish)

IMT 2020

International Mobile Telecommunications-2020

InH

Indoor Hotspot

ITU-R

International Telecommunication Union-Radiocommunication Sector

ITU-R SG3

ITU-R Study Group 3

KT Corporation South Korea Telecommunication company 
LNA

LO

$\log +\mathbf{L o g}$

$\log +\log +\log$

LOS

LPM

LTE

METIS

MIMO

MINECO

MINETAD

MiWEBA

mmMAGIC

MORSE

MPM

MultiEXCELL

NLOS

NTT DOCOMO

NYU

O2I

OD

ODU

OEM

OLOS

ONERA

PDF

PIMT

PL

PLE

PLL

PLO

ppm

Pwr+Log
Low Noise Amplifier

Local Oscillator

Two log-normal distribution combination

Three log-normal distribution combination

Line-Of-Sight

Laser Precipitation Monitor

Long-Term Evolution

Mobile and wireless communications Enablers for the Twenty-twenty Information Society

Multiple Input Multiple Output

Ministry of Economy and Competitiveness (acronym in Spanish)

Ministry of Energy, Tourism and Digital Agenda (acronym in Spanish)

Millimetre-Wave Evolution for Backhaul and Access

Millimeter-wave based Mobile radio Access network for fifth Generation Integrated Communications

Model for Rainfall Statistics Estimation

Millimeter-Wave Propagation Model

Multi-Exponential Cell Model

Non-Line-Of-Sight

Nippon Telegraph and Telephone company

New York University

Outdoor to Indoor propagation

Orbital Diversity

Outdoor Unit

Orbit Ephemeris Message

Obstructed-Line-Of-Sight

Office National d'Etudes et Recherches Aérospatiales (in French)

Probability Distribution Function

Propagation Impairment Mitigation Techniques

Path Loss

Path Loss Exponent

Phase-Locked Loop

Phase-Locked Oscillator

Parts per million

Power-law and Log-normal distribution combination 
PXI

Quadriga

RAOBS

RF

RH

RMS

RSPG

SD

SDR

SHF

SIMO

SNR

SST

SVD

SYNOP

TD

TDP

TLE

UCLouvain

ULPC

UPM

UPS

USA

UT

WINNER

WMO

WRC

XPD

XPR

ZTD
PCI extensions for Instrumentation

Quasi-Deterministic Radio channel Generator model

Radio-sound observations

Radio Frequency

Relative Humidity

Root Mean Square

Radio Spectrum Policy Group

Site Diversity

Software Defined Radio

Super High Frequency (3-30 GHz frequency range)

Single Input Multiple Output

Signal to Noise Ratio

Synthetic Storm Technique

Singular Value Decomposition

Surface synoptic observations

Time Diversity

Technological Demonstration Payload

Two-Line Elements

Université Catholique de Louvain (in French)

Up-Link Power Control

Universidad Politécnica de Madrid (in Spanish)

Uninterruptible Power Supply

United States of America

User Terminal

Wireless world Initiative New Radio

World Meteorological Organization

World Radiocommunication Conference

Cross Polar Discrimination

Cross Polarization Ratio

Zenith Tropospheric Delay 


\section{INDEX}

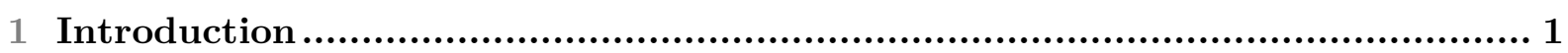

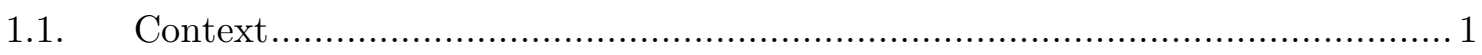

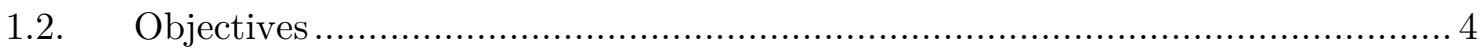

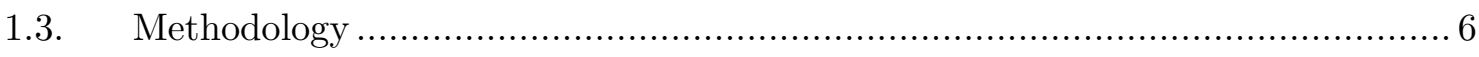

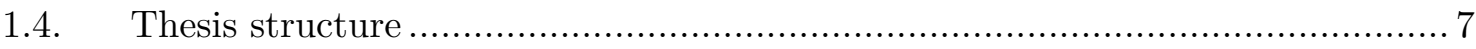

2 Propagation effects in satellite links .................................................. 9

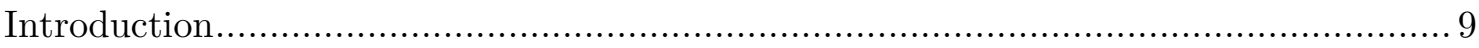

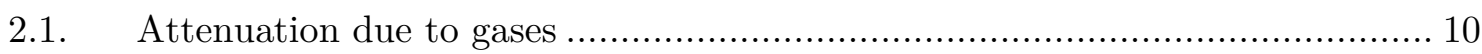

2.1.1. Gas attenuation prediction models ................................................... 12

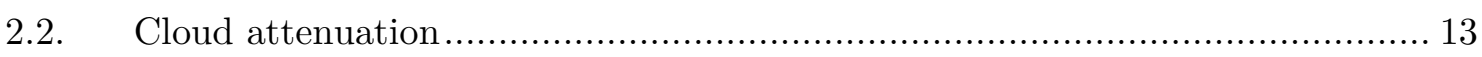

2.2.1. Classification and characteristics of clouds ........................................... 13

2.2.2. Cloud attenuation prediction models ...................................................... 13

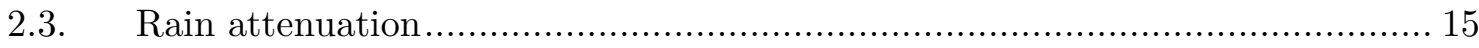

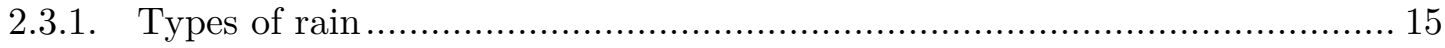

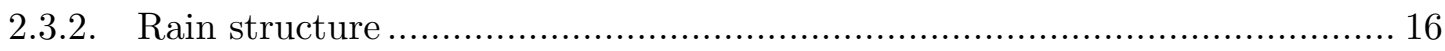

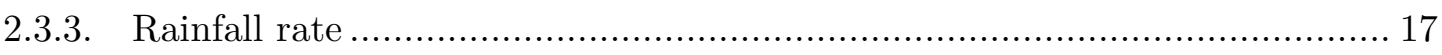

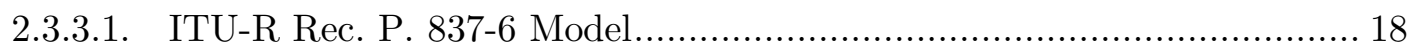

2.3.3.2. MORSE Model ................................................................................ 18

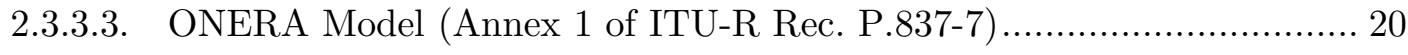

2.3.4. Rain attenuation prediction models....................................................... 22

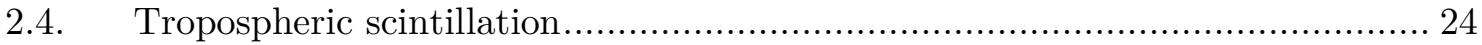

2.4.1. Tropospheric scintillation prediction models........................................... 25

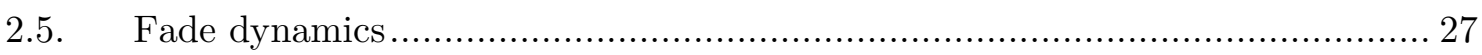

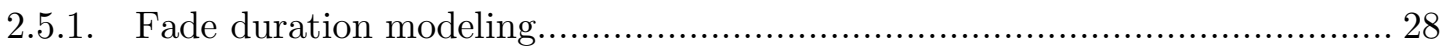

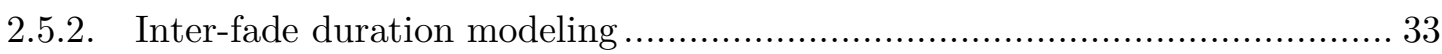

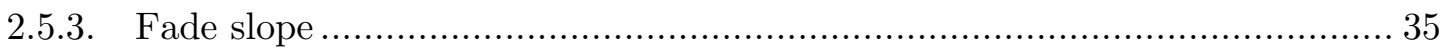

2.6. Propagation impairment mitigation techniques (PIMT): Diversity ................. 38

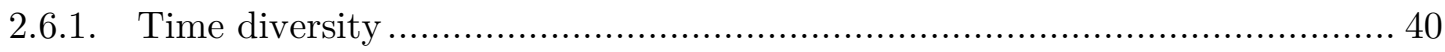

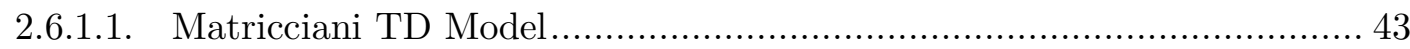

2.6.1.2. Joint Probability Model ................................................................ 43

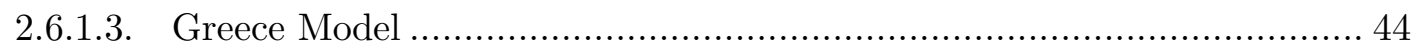

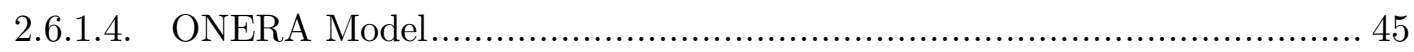




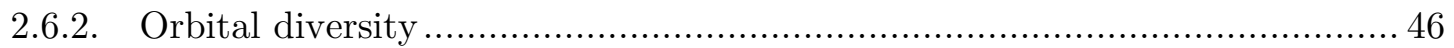

2.6.2.1. Matricciani OD Model................................................................... 47

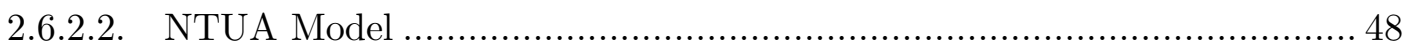

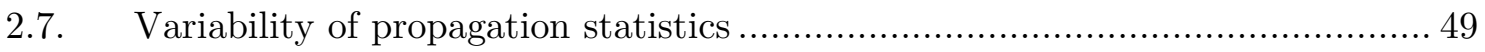

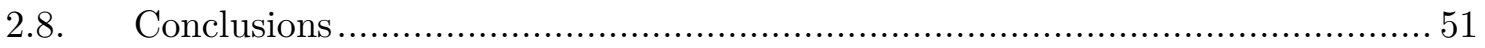

3 Millimeter-wave indoor channel modeling ........................................... 53

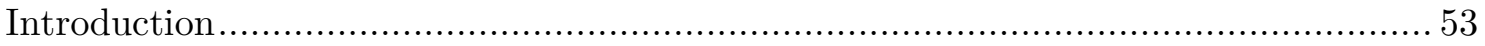

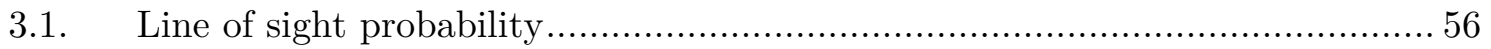

3.2. General large-scale path loss models ............................................................ 57

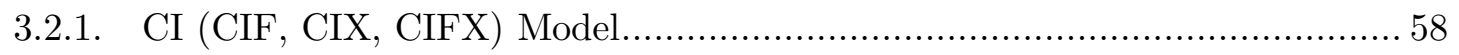

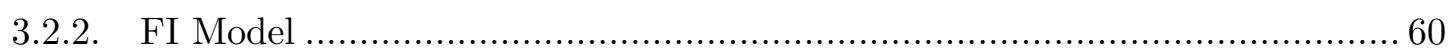

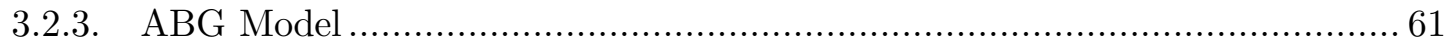

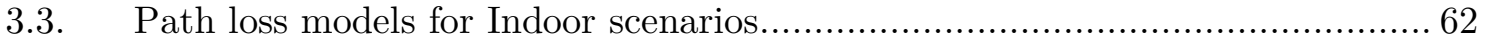

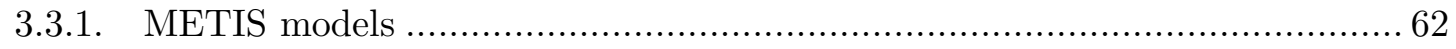

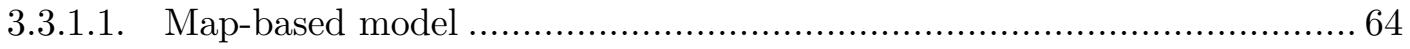

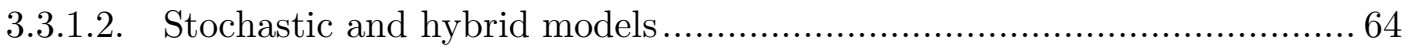

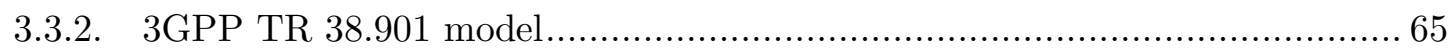

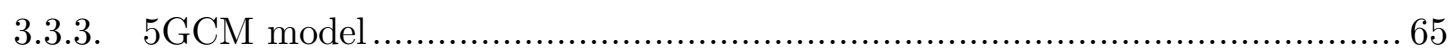

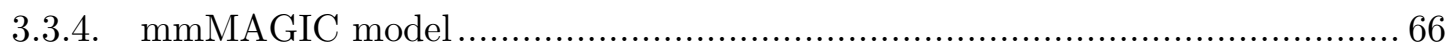

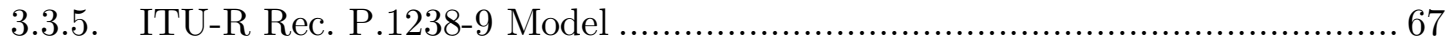

3.4. Multiple-Input Multiple-Output (MIMO) fundamentals .............................. 68

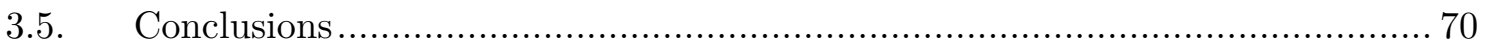

4 Experimental equipment and data processing ...................................... 73

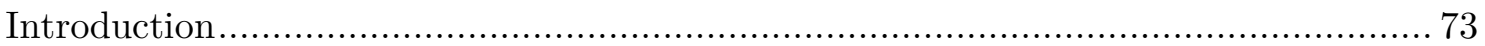

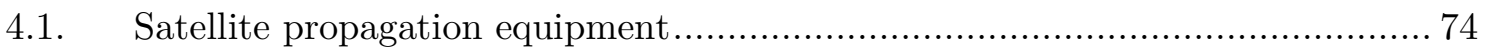

4.1.1. Ancillary meteorological instruments..................................................... 74

4.1.2. Eutelsat KA-SAT satellite propagation receiver ..................................... 76

4.1.3. Alphasat satellite propagation experiment ............................................. 78

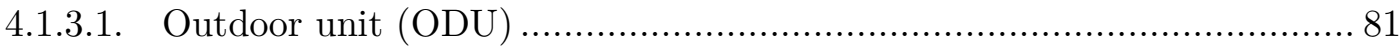

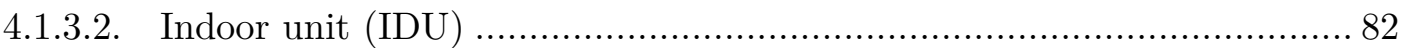

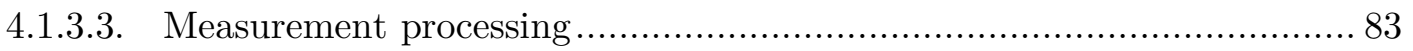

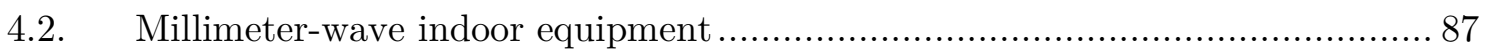

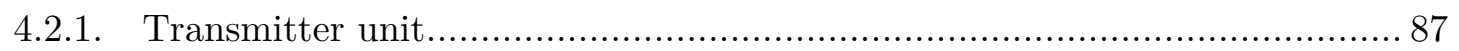

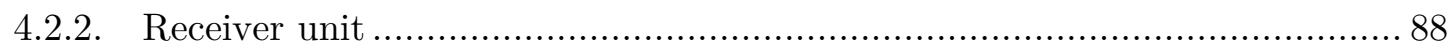


4.2.3. Data Processing.............................................................. 91

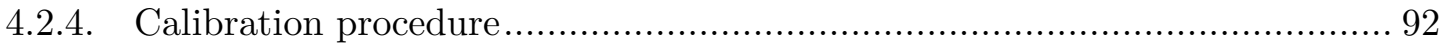

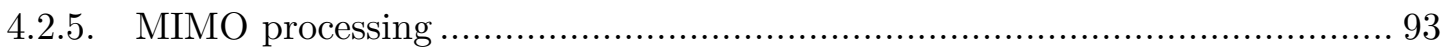

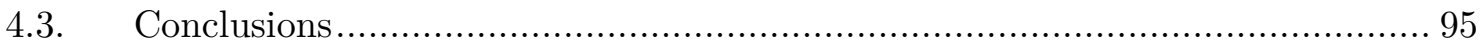

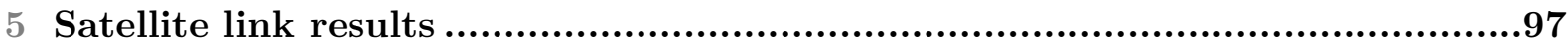

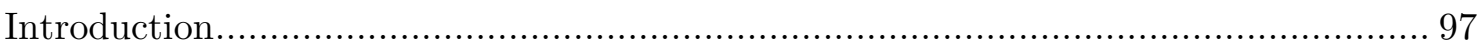

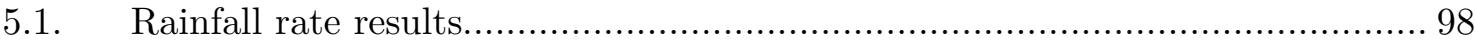

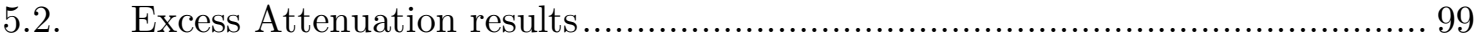

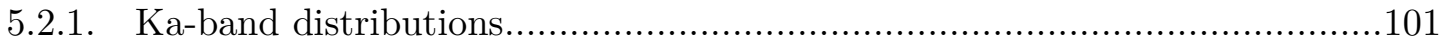

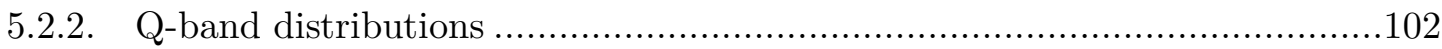

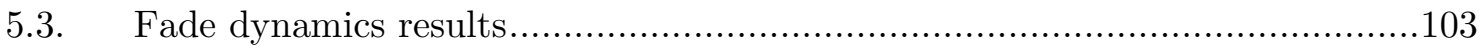

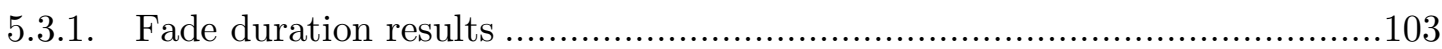

5.3.1.1. Comparison with models ............................................................103

5.3.1.2. FD probability of occurrence modeling...........................................106

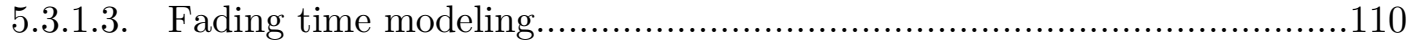

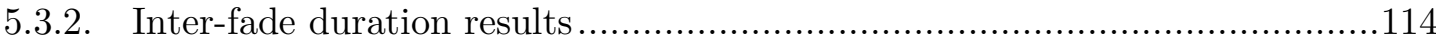

5.3.2.1. IFD probability of occurrence modeling ........................................115

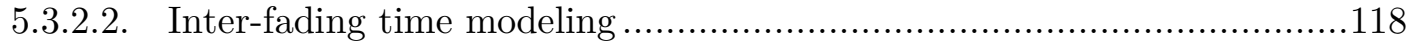

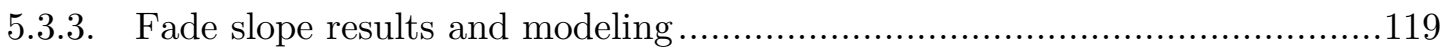

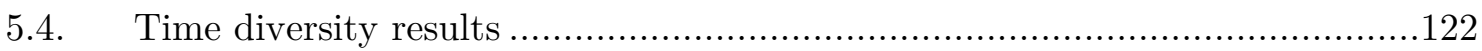

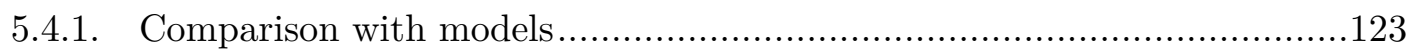

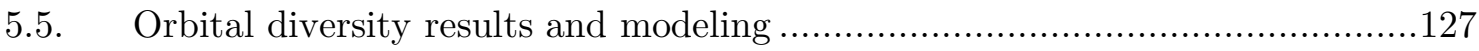

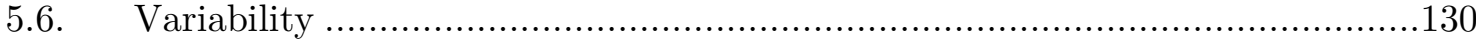

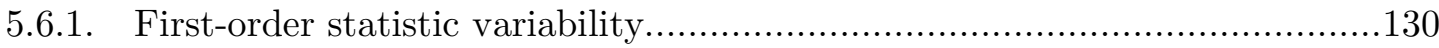

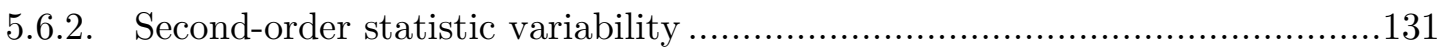

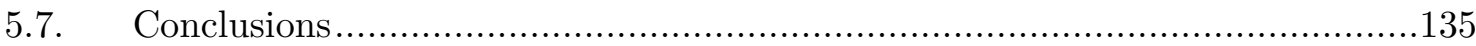

6 Millimeter-wave Indoor channel results ..........................................139

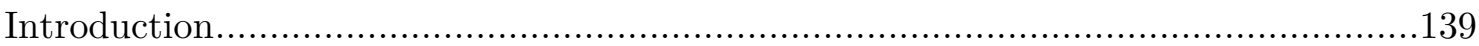

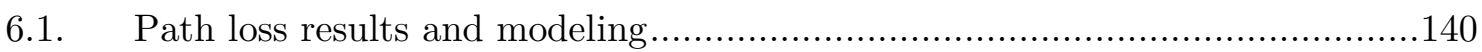

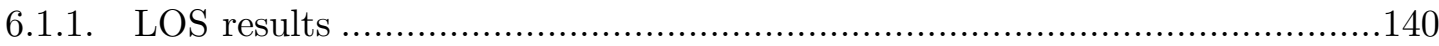

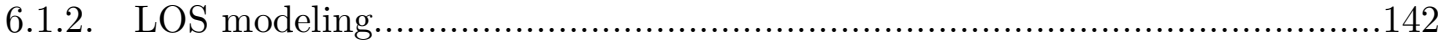

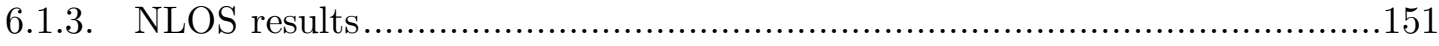

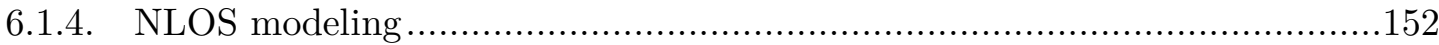

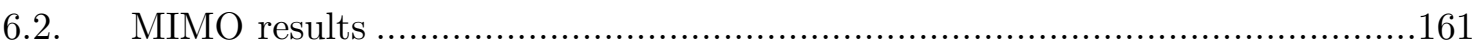




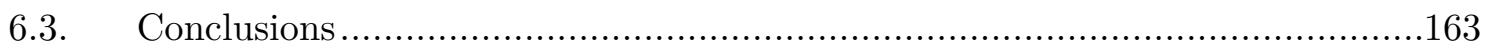

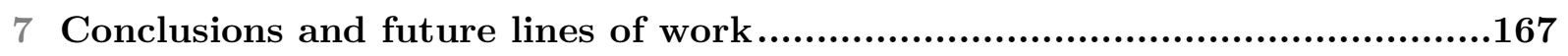

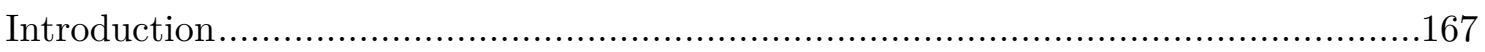

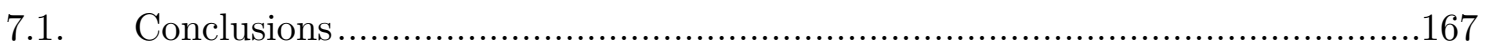

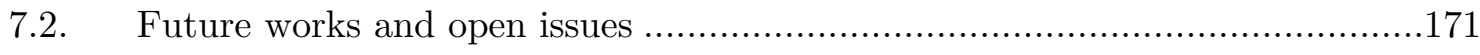

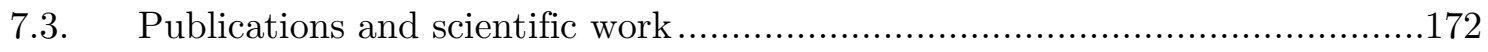

Annex 1 ONERA Time diversity model ................................................177

Annex 2 Procedure for obtaining the fading time distribution from a $\log +\log$ probability of occurrence distribution ......................................179

Annex 3 Total Attenuation results ..................................................181

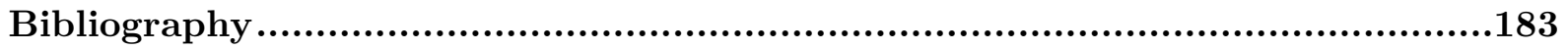




\section{1}

\section{Introduction}

This Doctoral Thesis has been carried out in the framework of the project TEC201457821-R named "Experimental characterization of atmospheric propagation and channel modeling in millimeter-wave bands, oriented to their future use in fixed and mobile 5G communication networks", funded by the Ministry of Economy and Competitiveness (nowadays Ministry of Economy and Business) of Spain. As part of the funding, the author has received the doctoral student grant BES-2015-071502 to work in the GTIC-Radiocommunication Research Group. The project has as general objective to produce advances in the characterization of the millimeter-wave propagation oriented to several radiocommunication systems. As the name of this Thesis states, in this work satellite and wireless communication systems at millimeter-wave frequencies are considered with the aim of contributing to the development of the project.

In this chapter, the technical context of this Doctoral Thesis is given. Moreover, the general and specific objectives and the actions needed to accomplish them are summarized. At the end, the document organization is outlined.

\subsection{Context}

The future spectral saturation of the frequencies in the SHF band $(3-30 \mathrm{GHz})$ in satellite communication systems, together with the crowding of the currently used bands (below 6 $\mathrm{GHz}$ ) and the increasing demand on high-speed data transfer in wireless communication systems have led to consider higher frequencies for allocating new applications for both systems. Millimeter waves (strictly-speaking, they are defined as signals with wavelength from 1 to $10 \mathrm{~mm}$, although signals with wavelengths up to $30 \mathrm{~mm}$ are informally included in the industry, thus covering the electromagnetic spectrum from 10 to $300 \mathrm{GHz}[\mathbf{1}]$ ) may ease 
that situation in spite of the technological challenges at these frequencies - which need to be overcome and well understood before their extensive deployment.

Millimeter-wave technologies are being used since some decades ago - they have been mostly used in radars, Earth exploration, point-to-point services and satellite communications - but nowadays the interest in them has grown exponentially in academic, industry and standardization groups due to their appealing to support high-speed data rates - up to several gigabits per second-, thanks to technological developments that have reduced the equipment cost. High speed is crucial in order to deploy new applications that may allow, for example, the transmission of high-definition un-compressed audio and/or video streams, enhancing the user experience and improving the service quality.

From a technical perspective, in higher frequency bands the path loss tends to increase and the signals that come from satellites are heavily attenuated by meteorological phenomena which occur in the troposphere, a reason to not consider them suitable for commercial purposes until short time ago. Recent developments on fade mitigation techniques have changed this perspective.

In satellite communications, a growth in the use of the Ka (17-31 GHz) and Q/V (40/50 $\mathrm{GHz}$ ) bands and above is expected, since lower bands like the Ku-band (10-15 GHz) will be saturated in a near future - they are now crowded and with a scarce additional capacityand few services are operating now at these higher bands.

Until now, some satellite services have been provided in the Ka-band around the globe, with some operators like HISPASAT [2], SES [3] and EUTELSAT [4] (among others) having transponders on-board of several satellites. On the other hand, the $\mathrm{Q} / \mathrm{V}$ band is not widely populated (at least for civilian and/or commercial services), as a matter of fact most of the satellites that have been operated in this band have had a research interest for a future band deployment, for example the ITALSAT satellite launched in 1991. Moreover, it is expected that commercial satellite services in Q/V band will be available in the decade of 2020. This band is expected to be used in the backbone network, reducing the number of required gateways by increasing the maximum achievable throughput and releasing bandwidth in the Ka band (allowing the use of this band only for the user terminals).

Although some propagation experiments have been carried out in the Ka and Q/V bands, the available results are not enough to fully characterize the slant-path propagation mechanism at these frequencies. Inserting a beacon with experimental purposes on a satellite payload is an expensive task that only few regional agencies or consortiums can afford. On the other hand, experimental receivers are also expensive and in many cases they are designed and built by the same research groups that operate them. The measurement campaigns must have high data availability for obtaining meaningful results and enabling thorough analyses. To accomplish that, high attenuation margins, especially at higher frequencies, are needed.

In wireless communications, there is high expectation with the fifth-generation wireless technology, known by the global telecommunication society as "5G". A 5G standardization has been developed by the 3GPP in Releases 15 [5] (which was frozen on September 2018) and 16 [6] (which is still open). The former addressed the first 5G standalone specifications 
and the latter is intended to complete the standardization of the different usage scenarios. Release 16 is under development and should be completed by December 2019 [6]. Although some tests have been carried out with existing networks, there is not any fully functional $5 \mathrm{G}$ network at this moment, but the first deployments are expected in the near future around 2020. Nevertheless, regarding propagation there has been a growth in the number of studies in the recent years, but still more measurement campaigns and analyses are useful and needed for a better propagation channel characterization. For $5 \mathrm{G}$, there is an agreement in the scientific community on the use of millimeter-wave frequencies - complementing the current use frequency bands below $6 \mathrm{GHz}$ - never used before in these kind of services; actually, the use of millimeter-wave frequencies is one of the four main demands identified in order to satisfy the $5 \mathrm{G}$ requirements (together with spatial consistency and mobility, diffuse versus specular scattering and very large antenna arrays $[\mathbf{7}])$. Higher frequencies allow wider bandwidths to satisfy service requirements in the transport network (backhaul), in the fronthaul and in the end-user services, but the associated propagation loss increases with respect to lower frequency bands.

Technical solutions based on MIMO (Multiple Input Multiple Output) and beamforming with a large number of antennas for compensating the propagation loss are becoming feasible at higher frequencies, due to the smaller antenna sizes and the ability of exploiting chip-scale antenna solutions. Besides, some commercial systems are already operating at millimeterwave bands, demonstrating a practical feasibility to build commercial transmitters and receivers $[\mathbf{8}]$.

The assessment of the spectrum availability and the harmonization of frequencies are crucial first steps to develop new services or standards. The objectives are to achieve global roaming and to seize the benefits of the economies of scale [9]. In order to ease these tasks, several studies regarding spectrum analysis are available such as [10] (in the Mobile and wireless communications Enablers for the Twenty-twenty Information Society (METIS) project framework) and [11] (in the project Millimeter-wave based Mobile radio Access network for fifth Generation Integrated Communications, mmMAGIC), among others. The Study Group 3 (SG3) of the International Telecommunication Union-Radiocommunication Sector (ITU-R) is carrying out studies about the propagation characteristics at higher frequency bands with the objective of having ready a proposal for the millimeter-wave spectrum allocation of the future systems by the next World Radiocommunication Conference (WRC), to be held in October-November 2019 in Sharm, el-Sheikh, Egypt. The ITU-R in the WRC-15 identified the millimeter-wave bands to be studied, ranging from 24.55 $\mathrm{GHz}$ to $86 \mathrm{GHz}[\mathbf{9}]$.

Based on the WRC-15, organizations of different geographical areas have started to identify suitable bands for their possible use in 5G. A very clear summary about the global situation is given in $[\mathbf{1 2}]$.

In Europe, it seems that the European Commission for Communications Networks, Content and Technology will follow the report of the Radio Spectrum Policy Group (RSPG) [13] that recommends the $24.25-27.5 \mathrm{GHz}$ band (named as the $26 \mathrm{GHz}$ band) as a pioneer band for an earlier implementation of $5 \mathrm{G}$ above $24 \mathrm{GHz}$. Also, the band $31.8-33.4 \mathrm{GHz}$ is seen as a promising one, since it can be made available easily by many European 
administrations. The $40.5-43.5 \mathrm{GHz}$ band is also considered as a viable option for $5 \mathrm{G}$ in the long-term. On the other hand, the RSPG does not give a strategy or road map for the upper part of the spectrum identified in the WRC-15.

The previous dispositions were taken into account by the former Secretary of State for the Information Society and Digital Agenda of the Ministry of Energy, Tourism and Digital Agenda (MINETAD, acronym in Spanish) of Spain [14], identifying in the 26-GHz band 400 $\mathrm{MHz}$ in the lower part and other $500 \mathrm{MHz}$ in the upper part for immediate use - the rest of the band is used by radio-links of the fixed service and networks of the mobile service. Also, the band is identified for pilot experiences, for commercial use and for harmonization and licensing in a near future. In the Spanish case, the other possible bands to be analyzed for their long-term use, according with the WRC-15, are the following: $37-40.5 \mathrm{GHz}, 45.5-47$ $\mathrm{GHz}, 47-47.2 \mathrm{GHz}, 47.2-50 \mathrm{GHz}, 50.2-50.4 \mathrm{GHz}, 50.4-52.6 \mathrm{GHz}, 66-76 \mathrm{GHz}$, and 81-86 $\mathrm{GHz}$.

With the objective of seizing the maximum of the millimeter-wave communication channel potentialities — for both, satellite and wireless communications - , it is necessary to carry out propagation experiments that allow the channel characterization, the validation of existing channel models and the development of new ones. It is very important to take into account that the impairments imposed by the medium to the signal at these frequencies are very relevant. An increase in frequency produces an additional attenuation to the satellite signal due to the impact of atmospheric phenomena such as rain, atmospheric gases, clouds, scintillation, etc.; in wireless communication, besides the frequency-dependent attenuation, adverse effect such as the multipath effect, diffraction and scattering also increase. These effects are present in the propagation scenarios either in line-of-sight (LOS) or non-line-ofsight (NLOS) conditions, and they have been partially addressed at these frequencies so far (although a growing number of papers and researches have been carried out in the recent years on that direction).

The GTIC-Radiocommunication Research Group of the Universidad Politécnica de Madrid (UPM) has as one of its objectives to contribute to the research, the knowledge and the dissemination of the radiocommunication technologies. At millimeter waves, the Research Group has a proven experience in satellite propagation experiments and campaigns, being relatively new its experience in wireless communications at these frequencies. This Thesis has included work on both lines of research, taking advantage of the availability of two satellite beacon receivers in the $\mathrm{Ka}$ and $\mathrm{Q} / \mathrm{V}$ bands and portable millimeter-wave wireless equipment suitable for propagation measurements.

\subsection{Objectives}

Having into consideration the previously described situation, the objective of this research is to contribute in the characterization and modeling of the millimeter-wave propagation channel - specifically for satellite links in the $K a$ and $Q / V$ bands and in indoor wireless links at frequencies (26 and $39 \mathrm{GHz}$ ) that will be used in future $5 \mathrm{G}$ systems - by collecting, processing and analyzing experimental propagation channel data. 
This objective has millimeter-wave communications as general scope, but it is divided into two lines of actions with their own specific objectives: satellite communications and indoor wireless communications, since the particular characteristics of both with respect to the propagation channel are very different.

Regarding satellite communications, a recent experimental campaign is being carried out by the Universidad Politécnica de Madrid (UPM) with one of the propagation payload of the Alphasat satellite of INMARSAT in the Q/V-band and the commercial KA-SAT satellite of EUTELSAT in the Ka-band.

The propagation in satellite communications takes place in line of sight conditions and most of the signal attenuation at the above mentioned frequency bands is due to atmospheric effects, particularly those that occur in the troposphere (although for mobile communications via satellite other effects such as obstruction and multipath must be taken into account). The specific objectives on this strand are:

- To derive and analyze the attenuation time series with very high data availability from the signals received by two beacon receivers (one in the Ka and another in the $\mathrm{Q}$ band), using some ancillary information provided by meteorological equipment.

- Modeling of fade dynamics (especially fade and inter-fade duration) and diversity techniques (time and orbital diversity) to characterize the propagation channel, since they have not been widely treated in previous works done by the Research Group. They allow knowing the behavior of the attenuation phenomena for a given scenario, necessary from an operator point of view for provisioning the fade margins and resource management.

- The analysis of the annual variability for first- and second-order statistics. Knowing how the propagation statistics vary in time allows better predictions. This is also another important issue that has not been addressed thoroughly by the GTIC in previous investigations.

The last two sub-topics are open issues for the satellite propagation community.

On the other hand, for indoor wireless communications the atmospheric effects are present but have a rather low relevance due to the relatively short path distances (in the order of hundreds of meters or less) currently envisaged for these applications and the absence of hydrometeors in indoor space. The scenario conditions (visibility range, surrounding obstacles, etc.), the multipath and the distortion that it produces are more relevant.

The available equipment can be used at the following bands identified as candidates for the provision of $5 \mathrm{G}$ mobile communications: from 24.25 to $27.5 \mathrm{GHz}$, from 31.8 to $33.4 \mathrm{GHz}$ and from 37 to $40.5 \mathrm{GHz}$ (only up to $40 \mathrm{GHz}$ ). The first and third bands have allocations to the mobile service on a primary basis whereas the second one may require additional allocations to the mobile service on a primary basis. These frequencies are of great interest since they constitute wide and contiguous segments of spectrum that may efficiently support very high data rates. Also, the measurements will be limited to the indoor scenario since it 
seems to be one of the most restrictive scenarios for the millimeter-wave propagation and most of the mobile usage occurs inside buildings. Here, the specific objective is:

- To gather and analyze path loss and MIMO measurements in indoor scenarios (both for LOS and NLOS conditions) at millimeter-wave frequencies with recently acquired equipment. This objective is twofold since the process of obtaining the measurements has been developed completely during this research and has not been presented in previous works.

Path loss measurements have been obtained for both 26 and $39 \mathrm{GHz}$, whereas MIMO measurements have been collected only at $39 \mathrm{GHz}$.

\subsection{Methodology}

The work methodology is oriented to the combination of theoretical aspects on millimeter-wave communications - both satellite and wireless indoor - with the experimental results that can be used to test and refine available models. The modeling of propagation phenomena from statistical analyses has a particular relevance, and the main contributions are expected from there.

In order to accomplish the general objective and the specific objectives, several tasks have been carried out within the aforementioned lines of research.

In the scope of satellite communications, data from propagation experiments in two frequency bands have been used: four years of beacon measurements from the Alphasat satellite $[\mathbf{1 5}]$ in the Q-band and five years of beacon measurements from the KA-SAT satellite in the Ka-band, having as research tasks:

1. A bibliographic study about the theoretical references on millimeter-wave propagation in satellite links and regarding the main propagation experiments carried out so far.

2. The reception and processing of the satellite beacon signals from KA-SAT at $20 \mathrm{GHz}$ and from Alphasat at $40 \mathrm{GHz}$.

3. The calculation of propagation first-order statistics on rainfall rate and rain attenuation.

4. The derivation of propagation second-order statistics: fade duration, inter-fade duration, fade slope, time diversity and orbital diversity.

5. The analysis of the time variability of the first- and second-order statistics.

6. Comparisons and fittings of the first- and second-order results with available models. Assessment of their accuracy and possible improvements.

For the sub-topic of indoor wireless communication, measurements in a corridor of one of the UPM buildings have been gathered in the last year. The research tasks are:

1. A bibliographic study about the available research results on the use of millimeterwave frequencies for $5 \mathrm{G}$ indoor wireless communications.

2. Gathering of propagation path loss measurements in indoor scenarios at the frequencies of interest (26 and $39 \mathrm{GHz}$ bands) for LOS and NLOS configurations. 
3. The fittings of the path loss measurements with general models in each configuration and the comparison with specific models.

4. The obtaining of the MIMO channel matrix and the use of the condition number as a channel capacity indicator.

\subsection{Thesis structure}

This document is structured in seven chapters and three annexes. After this introductory chapter, the main satellite propagation phenomena (particularly rain attenuation) and other topics that are treated in this Thesis (i.e. fade dynamics, time and orbital diversity and variability) are presented in Chapter 2. Since the Research Group has a long experience on this scope with several Doctoral Theses focused on satellite-to-Earth propagation, the aim here is to present the main topics that will serve for further analyses not carried out in those previous works. For this reason, first-order statistics of effects such as gas, or cloud attenuation and tropospheric scintillation are lightly addressed and the main attention is given to rain and the attenuation that it produces. Regarding second-order statistics, fade dynamics is treated in depth, since it is one of the most important issues in satellite propagation and in it a larger contribution is expected. Moreover, some diversity techniques (time and orbital diversity) that can be analyzed with the available equipment are presented. Procedures for assessing the variability of the first- and second-order statistics are also discussed.

Chapter 3 addresses the theoretical background on the millimeter-wave channel modeling. First, general large-scale path loss models are presented; these are the bases of the models that have been developed for indoor scenarios at millimeter-wave frequencies, which are also described. Moreover, the basic concepts on MIMO channel modeling are given. Although MIMO topic is the focus of several researches published in the literature, here only the part regarding the channel matrix and condition number estimation is addressed, since these are the issues considered in the experimental work.

All the equipment used in this research, either for the satellite or the indoor wireless propagation experiments, is described in Chapter 4, detailing the main data processing characteristics. The KA-SAT beacon receiver is presented in less detail, since it has been reused from a previous experiment, which has been addressed thoroughly in previous works. On the other hand, the Alphasat experiment and the indoor equipment are treated in a deeper way. For the indoor setup, a main point is the calibration procedure, which is the basis of all measurements. Also, some special considerations to derive MIMO measurements from the available setup are presented.

Chapter 5 records the main results for satellite propagation in the Ka- (KA-SAT) and Qbands (Alphasat). These are based on excess attenuation time series, and include fade dynamics comparison with models and the fade and inter-fade duration modeling efforts. Furthermore, time and orbital diversity results are given for the four-year concurrent period of measurements for both experiments. Time diversity is assessed directly from the attenuation results of both experiments, and a specific frequency scaling procedure is used for 
evaluating orbital diversity in the Q-band. Variability is assessed both for first and second order statistics.

The LOS and NLOS path loss and MIMO results relative to the wireless indoor propagation channel are collected in Chapter 6. Path loss is assessed at 26 and $39 \mathrm{GHz}$. For the LOS measurements, several transmitter-receiver positions in a corridor have been used. NLOS measurements were gathered from three passes along the interception of two corridors with a corner as the main point of diffraction, albeit they are presented all together. The millimeter-wave indoor path loss models were also tested (either for LOS or NLOS conditions). MIMO measurements were obtained in an office-corridor scenario with LOS and NLOS conditions at $39 \mathrm{GHz}$.

The Thesis conclusions and future lines of work are outlined in Chapter 7 in which the main publications in journals and conferences and scientific works developed in the framework of this research are also presented.

In the Annexes some relevant issues not included along the document but necessary to complement the results are given. Annex 1 presents the step-by-step ONERA (Office National d'Etudes et Recherches Aérospatiales) time diversity model, which is only available so far from an ESA (European Space Agency) project. The possibility of using the software that implements this model is kindly appreciated. Annex 2 gives some intermediate steps of a procedure for obtaining the fading time distribution from a two log-normal function probability of occurrence distribution. Total attenuation results for Alphasat are collected in Annex 3.

At the end of this document, the list of bibliographic references is included. 


\section{2 \\ Propagation effects in satellite links}

\section{Introduction}

Radio wave propagation at high frequencies is mostly conditioned by impairments caused by the propagation medium. In LOS satellite links, besides the free space path loss, additional losses that attenuate the signal strength and cause fades take place in the propagation path as a consequence of a variety of tropospheric phenomena (such as rain, clouds, gases, tropospheric scintillation, etc.). The statistical distributions of these phenomena and the attenuation that they produce are usually referred to as first-order statistics.

The propagation loss is not just a simple constant term to be taken into account in the link budget. Due to its high variability, it is necessary to know its statistical behavior. Firstorder statistics may not provide all the information required to exploit all the potentialities of frequencies higher than $18 \mathrm{GHz}(\mathrm{Ka}, \mathrm{Q} / \mathrm{V}, \mathrm{W}$ bands) and the possibilities of mitigating rain attenuation effects. In these frequency bands, the desired availability may not be guaranteed with a conventional design, so the introduction of Propagation Impairment Mitigation Techniques (PIMT) in the systems is necessary for mitigating in real time the negative impact of propagation events and to ensure in that way certain link availability and an adequate quality of service.

Besides the conventional design problems, the system flexibility must be considered in the adaptive transmissions (obtained by: code or frequency variations, dynamic allocation of 
resources, reconfigurable antennas, etc.). Channel propagation dynamics is therefore a key element in the system design and must be taken into consideration in the PIMT control loop (element able to follow the signal variations, especially the slow component of attenuation, and to evaluate the envelope of the fast variations).

The ITU-R in its Recommendation P. 618-13 [16] points out the following as the main signal fade contributions:

- Attenuation due to atmospheric gases.

- Attenuation due to clouds, rain and other precipitations.

- Scintillation and multipath effects.

- Attenuation due to sand and dust storms.

- Antenna mismatches.

- The decrease of antenna gain due to incoherence in the wave front.

All of these have geographical, elevation angle and frequency dependencies. Only gas, cloud and rain attenuation and -in a less amount- tropospheric scintillation have importance for elevation angles above $10^{\circ}$ (as it is the case of the UPM receivers).

There are a variety of ways to quantify the fluctuation of the signal level along the propagation path, one of them is attenuation. In absolute terms, the total attenuation in a propagation path is the difference between the signal level that could reach the terrestrial station if the space between it and the satellite is vacuum and the signal level that is actually received. Then, total attenuation is the sum of all attenuation mechanisms that act along the propagation path. On the other hand, excess attenuation is the difference between the signal level in clear-sky condition (in the case of this Thesis, clear sky is understood as a condition in which only attenuation by gases is present) and the signal level measured when relevant events of attenuation like rain and clouds occur [17]. Then, total attenuation is the sum of excess attenuation and gas attenuation.

For all these reasons, this chapter has as objective to analyze the literature references about the main propagation effects in millimeter-wave for satellite links. Since previous works [18] [19] carried out at the GTIC-Radiocommunication Research Group have been focused on this topic thoroughly, the objective is limited to the main concepts and references on firstorder (gas, cloud and rain attenuation and tropospheric scintillation) and second-order (fade duration, inter-fade duration and fade slope) propagation statistics that can provide the needed background for further analysis. Also, the main PIMT are described and the diversity techniques that can be evaluated with the available equipment and data (time diversity and orbital diversity) are analyzed in depth. Moreover, the methods to assess the variability of certain statistics are addressed.

\subsection{Attenuation due to gases}

At microwave and millimeter-wave frequencies, oxygen and water vapor are the main responsible of atmospheric absorption, specifically the absorption of electromagnetic energy 
produced by the interaction of an incident electric or magnetic field wave with a molecule with an electric or magnetic dipolar moment. This phenomenon occurs at specific frequencies (determined by spectral lines).

Figure 2.1 shows the zenith attenuation produced by gases according with the method described in Annex 2 of the Recommendation ITU-R P. 676-11 [20]. The curves represent the gas attenuation that can be observed in a vertical line from the sea level through a reference standard atmosphere in an Earth-to-space link.

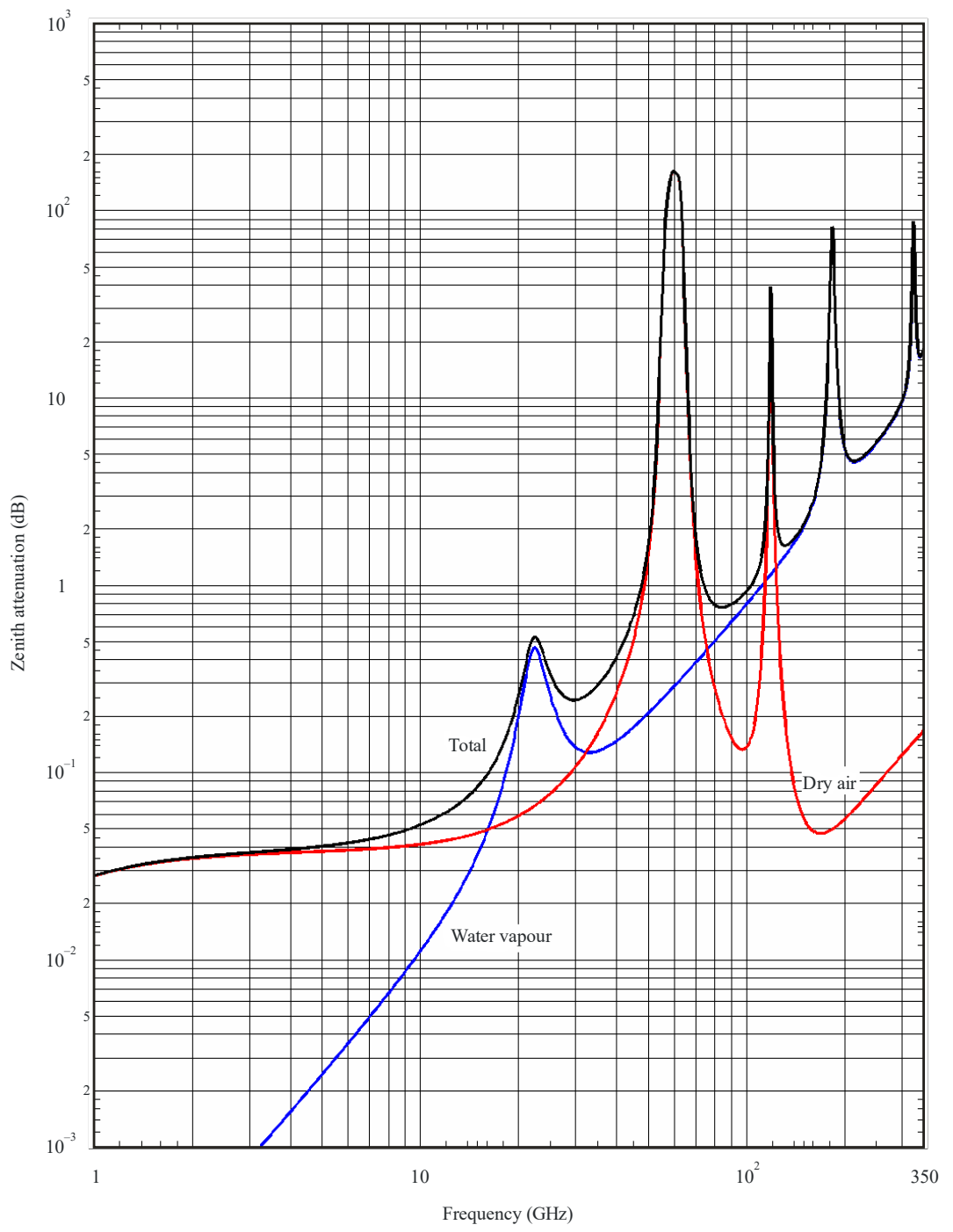

Figure 2.1. Total zenith attenuation due to atmospheric gases [20].

The curve Dry air corresponds to an atmosphere without water vapour content, the curve Water vapour corresponds to a surface density of $7.5 \mathrm{~g} / \mathrm{m}^{3}$, and the curve Total represents the combination of the previous ones in a standard atmosphere with a surface pressure of $1013 \mathrm{hPa}$ and temperature of $15^{\circ} \mathrm{C}$.

There are a number of critical frequencies where resonance absorption occurs, with the corresponding increase in the base level of attenuation with frequency.

Oxygen attenuation almost has a constant value with time and location due to its uniform concentration in the atmosphere - although it suffers small variations with 
temperature - and has several spectral lines where strong absorption occurs. It has an isolated absorption line at $118.7 \mathrm{GHz}$ and a series of absorption lines between 50 and 70 GHz. In the lower part of the atmosphere the oxygen lines span as consequence of the pressure and form a single absorption spectrum around $60 \mathrm{GHz}$. The spread of the lines at higher frequencies due to the pressure produces significant effects at millimeter-wave bands, because the absorption in these lines extends its effect to lower and higher frequencies. Moreover, the magnetic moment of the oxygen molecule produces a line at zero frequency in the absorption spectrum, a phenomenon known as Debye or non-resonant spectrum.

Water vapor attenuation is more variable because of the great fluctuation of the water vapor content in the atmosphere that can be seen in vertical humidity profiles. Absorption occurs in three lines below $350 \mathrm{GHz}$ : a weak line at $22.2 \mathrm{GHz}$ and more intense lines at 183.3 and $323.8 \mathrm{GHz}$. Overall, gas attenuation progressively increases with frequency, but reaches its peaks in these lines, and is more stable below $10 \mathrm{GHz}$. Absorption caused by other gases can be disregarded in comparison with the oxygen and water vapor absorption.

\subsubsection{Gas attenuation prediction models}

The atmospheric gas attenuation can be described using a physical approximation such as the Liebe model [21] — also known as MPM (Millimeter-Wave Propagation Model), which is considered as a very complete and valid model from 1 to $1000 \mathrm{GHz}$ - or others such as the ITU-R [20] and the Salonen models [22]. Other models use certain specific parameters, as the Rosenkranz model $[\mathbf{2 3}]$ does with the absorption level produced by the water vapor.

The Liebe model develops in a precise manner the calculation of the air refractivity for frequencies between 1 and $1000 \mathrm{GHz}$, but has high computational cost, requires vertical data of meteorological parameters and its degree of accuracy must be carefully tested. This model is used often as reference in model comparisons, or to obtain propagation parameters such as the absorption coefficient or the equivalent attenuation height.

The Recommendation ITU-R P. 676-11 [20] provides two models to estimate gas attenuation: one is the Liebe model, presented in its Annex 1 as a "line-by-line calculation of gaseous attenuation", the other is presented in Annex 2 as the "approximate estimation for gaseous attenuation in the frequency range 1 to $350 \mathrm{GHz}$ " and can be used for a rapid and approximate estimation.

The Salonen model predicts the gas attenuation for the Ku-band and above by using as inputs climatological parameters that are available in the radio-climatological maps of the ITU-R. In the literature there is a model to predict oxygen attenuation [24] and other to predict water vapor attenuation $[\mathbf{2 5}]$.

A recent model for oxygen attenuation was presented in [26] by Luini and Riva, in which they take advantage of meteorological data from high-resolution radio-sound observations (RAOBS) collected worldwide in order to develop a simplified but yet accurate procedure. This model uses as input data the mean yearly ground temperature and the complementary cumulative distribution function (CCDF) of the ground water vapor content (both available in ITU-R global maps [27] [28]). 


\subsection{Cloud attenuation}

Clouds can be defined as a mixture of very small water drops, ice crystals or both, that are suspended above the surface (usually in the troposphere, although they can also be found in the stratosphere). Commonly, clouds that are not associated to precipitations are composed by small water droplets with less than $30 \mu \mathrm{m}$ radius (the diameter limit for liquid particles to be considered as part of a cloud is around $200 \mu \mathrm{m}$, bigger drops are considered as drizzle or rain $[\mathbf{2 9}])$.

Cloud attenuation can be the main propagation impairment in systems with low availability. Moreover, there are clouds entirely formed by ice particles, which do not produce a meaningful attenuation although they can produce wave depolarization.

The cloud attenuation prediction basis is the theory of scattering of radio waves for isolated particles [30], specifically the Rayleigh approximation of the scattering theory of Mie for particles smaller than $100 \mu \mathrm{m}$ (which considers cloud attenuation independent of the drop size distribution, DSD, up to $300 \mathrm{GHz}$, depending only on the liquid water content and temperature). This scattering model allows to assess with high degree of accuracy the attenuation produced by the water drops presented in the clouds, but its application is not immediate due to the physical complexity of cloud modeling and the scarcity of accurate data.

\subsubsection{Classification and characteristics of clouds}

As meteorological events, clouds have been vastly studied at macroscopic level (dynamic of clouds movements) and at microscopic level (the micro-physic of clouds as the fundamental of its formation), that is why a wide specialized literature is available $[\mathbf{3 1}],[\mathbf{3 2}]$, $[33],[34],[35]$.

The World Meteorological Organization (WMO) defines in [29] certain criteria and methods to classify clouds by taking into account the shape and appearance of clouds (this is the most commonly used way in the radio-propagation community).

The three levels (or étages) cloud classification (high, medium, low) is usually employed in meteorology, with its categories explained in [36].

\subsubsection{Cloud attenuation prediction models}

A cloud can be considered as a scattering medium, formed by a population of spherical particles with diameters $D(\mathrm{~mm})$ and a drop size distribution $n(D)\left(\mathrm{m}^{-3} \mathrm{~mm}^{-1}\right)$, that occupies a certain volume. Several cloud models use the Rayleigh approximation for small drops by relating the specific attenuation with the liquid water content. The specific attenuation $\gamma_{c}$ (attenuation by unit of distance, in $\mathrm{dB} / \mathrm{km}$ ) generated by a cloud can be estimated as:

$$
\gamma_{c}=4.343 \cdot 10^{3} \int \sigma_{e}(D) \cdot n(D) d D \quad[\mathrm{~dB} / \mathrm{km}]
$$

where $\sigma_{e}$ is the cloud particle effective cross-section (in $\left.\mathrm{m}^{2}\right)$. 
Due to the complexity of modeling $n(D)$ and the lack of experimental data, some semiempirical models relate cloud attenuation with atmospheric parameters easier to estimate, such as the liquid water content $w_{l}\left(\mathrm{~g} / \mathrm{m}^{3}\right)$. In the frequency range where the Rayleigh approximation is valid, the specific attenuation depends only on the liquid water content, being independent of the DSD. In order to estimate $w_{l}$, a layered atmosphere is assumed and $w_{l}$ can be calculated in each layer in two steps:

1. The existence of a cloud level is identified or detected in the vertical profile by means of a detection algorithm, commonly based on a certain relative humidity threshold (RH) or a critical humidity function.

2. $w_{l}$ is later calculated using the model formula and can be used in different cloud attenuation models.

The Decker model [37] detects clouds by comparing the relative humidity in each layer of the atmosphere with a constant threshold (a value of $95 \%$ in the original paper). The estimation of $w_{l}$ is done with a very simple formula.

The Salonen-Uppala model [38] is based on radio-sounding measurements from more than 40 European stations and data from the European Center for Medium Range Weather Forecast (ECMWF), providing vertical profiles of temperature and humidity. This model is considered one of the most complete because it allows cloud detection (using an $\mathrm{RH}$ threshold, information about the atmospheric layer and surface pressure and two other empirical parameters), provides an estimation of $w_{l}$ (it uses an equation that depends on cloud height and temperature) and calculates the attenuation through the slant path.

Mattioli in [39] proposed another model (CldMod), in which some characteristics of the Salonen-Uppala model are improved, and a more real approximation of $w_{l}$ is done by considering additional effects such as the presence of dry air, precipitations, cooling and warming processes and the use of ceilometer ${ }^{1}$ measurements.

Other models estimate attenuation by using methods based on experimental propagation results. One of these is the Altshuler and Marr method [40], based in the regression of measurements from 15 to $35 \mathrm{GHz}$ and elevation angles from $1^{\circ}$ to $20^{\circ}$. In it, a linear regression of zenith attenuation as a function of surface absolute humidity (in $\mathrm{g} / \mathrm{m}^{3}$ ) was performed. The Dintelmann and Ortgies model [41] is based on the regression of surface humidity and temperature measurements obtained with a radiometer at 20 and $30 \mathrm{GHz}$. More recently, Luini and Capsoni [42] proposed a simple but precise model to calculate cloud attenuation in slant paths in the 20 to $200 \mathrm{GHz}$ range. The main feature of this method is the use of a liquid water mass absorption coefficient independent of the location and dependent only of the frequency.

Dissanayake, Allnutt and Haidara [43], [44] proposed a model that is based on the average properties of four cloud types and their occurrence probabilities (which can be obtained using surface synoptic observations, SYNOP). Moreover, the ITU-R in [45]

\footnotetext{
${ }^{1}$ Instrument used to determine the height from the ground to the cloud base and the visibility by means of a laser or another source of light, which transmits a low power pulse at high speed and detects the reflection retro-dispersed by the clouds and the gases that are above the instrument.
} 
recommends a method to estimate the attenuation in slant paths from statistical information of the total content of the liquid water column (in $\mathrm{g} / \mathrm{m}^{3}$ ) reduced to a $0^{\circ} \mathrm{C}$ temperature.

\subsection{Rain attenuation}

The attenuation due to rain is one of the main impairments in satellite links at millimeter-wave frequencies. This is caused by the scattering and absorption of the signal in the rain drops. At lower frequencies, the fades of the signal due to rain are less deep and the attenuation can be compensated with an acceptable link margin. However, deeper fades at millimeter-wave bands do not allow the application of a fixed margin. That is the reason to develop methods oriented to reduce the outage in the communication produced by rain fades. These methods are intended to mitigate as far as possible rain attenuation effects (as well as the attenuation caused by other effects) and receive the name of Propagation Impairment Mitigation Techniques (PIMT). Some of them will be presented later.

Rain will be treated more thoroughly than other phenomena because of its relevance in the experimental measurements, either for the Ka- or Q-band.

\subsubsection{Types of rain}

Liquid precipitations are associated intuitively with rain, even though its classification could be more specific using certain terms as the rain intensity - the amount of rain that falls in certain time, also known as rainfall rate. Traditionally, rain events are classified as:

- Stratiform precipitation. The precipitation is horizontally layered with rain up to the bright band (narrow zone of about $300 \mathrm{~m}$ around the $0^{\circ} \mathrm{C}$ isotherm with high reflectivity, formed by ice particles and molten ice). Ice can be found up to $7 \mathrm{~km}$ and ice crystals up to $9 \mathrm{~km}$. This type of precipitation is characterized by low rain intensity (less than $10 \mathrm{~mm} / \mathrm{h}$ ) and long duration events (even more than 1 hour).

- Convective precipitation. Characterized by strong ascendant and descendant streams extended in the troposphere region. It corresponds to rains easily localized in space - up to several kilometers - and with a short duration - tens of minutes. It is observed in meteorological radars as vertical columns with high refractivity.

- Monsoon rain. Sequence of events with intense convective precipitations followed by stratiform precipitations. The rain zones usually are up to $50 \mathrm{~km}$ wide and hundreds of kilometers long. This type of events produces several hours of intense rain.

- Tropical storm. It comprises a wide area, usually around hundreds of kilometers, characterized by several spiral bands that end in a very intense precipitation region around a central calm region called the "eye of the hurricane". The spiral bands have several regions with convective precipitation.

In temperate zones there are only stratiform and convective precipitations, although they do not appear isolated, commonly they occur combined and it is hard to define an exact limit for each one. 
In the center of the Iberian Peninsula, with a continental climate, dry and hot summers and cool winters with some precipitations, the mean precipitation level is $440 \mathrm{~mm}$ per year, spring and autumn being when it rains most. The stratiform precipitations occur at the end of autumn, in winter and at the beginning of the spring. Convective precipitations are more frequent in spring, summer and at the beginning of autumn.

\subsubsection{Rain structure}

For predicting rain attenuation, it is necessary to characterize properly its structure along the propagation path. As rain has a high time and geographical variability, it is almost impossible to characterize it in every moment and in every point on the path. A good approximation can be obtained by using adequate equipment, such as telemetry onboard satellites, meteorological radars, disdrometers and rain gauge networks covering an area. Measurements from these instruments confirm that short intense rain events can be found in long events of light rain and that there are smaller regions of intense rain in wider areas of light rain.

Rain attenuation prediction models use reduction factors that have into account the rain spatial and temporal in-homogeneities in the slant path. So, information on the vertical and horizontal structure of the precipitation, its height, the rain DSD and temperature are required.

A group of rain cells distributed in an area of interest is usually utilized to model the horizontal structure of the precipitation intensity [46]. A rain cell is defined as the area in which the rain intensity exceeds certain threshold of about 2 to $10 \mathrm{~mm} / \mathrm{h}$. A residual rain with a uniformly distributed intensity exists in the space left between cells. Rain cells are characterized by the following parameters: dimension, shape, orientation, maximum intensity, and direction and velocity of movement. Among the commonly used rain cell models are: the EXCELL Model (EXponential CELL Model) [47], which uses circular cells and an exponential decreasing rain intensity pattern inside the cell in comparison with its maximum value; the HYCELL [48], that uses elliptical cells and a combination of Exponential and Gaussian distributions to model the rain intensity; and the MultiEXCELL [49], that generates rain regions for simulating the natural process of aggregation of cells.

The vertical structure of rain can be modeled in a simple manner as a vertical homogeneous and cylindrical cell that is extended from the Earth surface to the $0^{\circ} \mathrm{C}$ isotherm height, although this procedure may overestimate the attenuation for regions with strong precipitations and high temperatures, like the tropics.

Some other characteristics of the vertical structure of rain are determined from radar images according to the precipitation type:

- Stratiform precipitations are characterized by small vertical movements of air and a thin horizontal layer with high refractivity - its width can go from $300 \mathrm{~m}$ up to $1 \mathrm{~km}$. This layer is composed mainly by ice crystals and melted ice crystals (this characteristic gives the name to the layer as melting layer, or bright band according with its shape in radar images) and produces an additional attenuation. The region above the melting layer is composed by a mixture of ice crystals that does not produce 
an important attenuation in frequencies lower than $60 \mathrm{GHz}$. The height at which occurs the transition between the bright band and the previously mentioned region is named as transition height and is closely related with the $0^{\circ} \mathrm{C}$ isotherm height.

- In convective precipitations, vertical column regions with high reflectivity can be found. In these regions there are strong vertical movements of air that mix the different particles. Thus, very cold rain drops can be found in some convective cells above the $0^{\circ} \mathrm{C}$ isotherm.

The rain height acts as the limit of the vertical extension that is taken to estimate the rain attenuation and in general does not match with the $0^{\circ} \mathrm{C}$ isotherm, although some attenuation models use the isotherm as a maximum height for stratiform rains. The ITU-R Rec. P.839-4 [50] recommends the use of data from a digital global map in regions where specific information of $h_{0}$ (the mean annual height of the $0^{\circ} \mathrm{C}$ isotherm above the sea level) is not available, and proposes an equation to estimate the value of the mean annual rain height of above the sea level, $h_{R}$ :

$$
h_{R}=h_{0}+0.36 \quad[\mathrm{~km}]
$$

Rain height can be higher than the estimated value for convective precipitation due to the presence of super-cooled liquid water drops in higher layer of the atmosphere. That is a reason of uncertainty in the correlation between the rain height and the isotherm height in this type of cells.

The effect of rain drops shape and size to the attenuation can be disregarded for frequencies below $10 \mathrm{GHz}$. One of the main reasons of the raindrops shape is the action of the aerodynamic forces on them when they fall to the Earth. For a bigger raindrop (a diameter higher than $4 \mathrm{~mm}$ ), it will be easier that its shape is distorted with respect to the spherical form.

In a rain event, the DSD is relatively stable despite the high variability in size, shape and orientation of the drops. Laws and Parson [51] were the first to determine the rain DSD in a reliable and experimental way; their distribution has a stable accuracy for rain drops with diameter longer than $0.5 \mathrm{~mm}$, although the original measurements only reach up to $50 \mathrm{~mm} / \mathrm{h}$ of rain intensity and must be extrapolated for higher values. Other rain DSD models have been proposed such as the negative exponential [52], [53]; the gamma [54] and the Weibull [55] functions.

\subsubsection{Rainfall rate}

The rainfall rate or rain intensity $R(\mathrm{~mm} / \mathrm{h})$ is one of the most important parameters in the rain characterization and gives the amount of precipitation that falls during a certain time interval. It is commonly expressed in $\mathrm{mm} / \mathrm{h}$ with an integration time interval of one minute. Rain events are classified as such according to a minimum value of $R$ (the ITU-R uses a $0.25 \mathrm{~mm} / \mathrm{h}$ value as the minimum threshold). This magnitude is measured directly from instruments such as rain gauges (the most commonly used instruments), disdrometers, and rain radars $[\mathbf{5 6}]$. 
If local rainfall rate measurements are not available, the rainfall rate distribution can be estimated by using some models based on large meteorological databases. From the three models that will be treated here the MORSE and ONERA models will be presented more in depth due to their relative novelty and that the ITU-R Rec. P. 837-6 Model [57] is well addressed in the literature. The ONERA model was adopted recently in Recommendation ITU-R P. 837-7 [58].

\subsubsection{ITU-R Rec. P. 837-6 Model}

This model [57] is based on the use of 40 years of meteorological data collected by the ECMWF. The ITU-R recommends the use of local long-term rainfall rate measurements if they are available, although it is necessary to have more than a 3 -year period to assure the statistical stability.

Rec. ITU-R P.837-6 provides global maps with the rainfall rate exceeded for the $0.01 \%$ of an average year that can serve as a first approximation. Moreover, there is a software application in the ITU web-page based on MATLAB [59], which allows the conversion of the rainfall rate distributions taken with an integration time of up to 60 minutes to rainfall rate distributions with an integration time of 1 minute. Statistics based on 1-minute integration time are more difficult to find at a global scale.

The model uses three variable $\left(P_{r 6}, M_{t}\right.$ and $\left.\beta\right)$ provided in supplementary files with a resolution of $1.125^{\circ}$ of latitude and longitude. The values at the specific coordinates can be found by means of a bilinear interpolation as it is described in Rec. ITU-R P.1144-7 [60]. The model outputs are the percentage probability of rain in an average year, $P_{0}$, and the rainfall rate distribution with 1-minute integration time.

\subsubsection{MORSE Model}

The MOdel for Rainfall Statistics Estimation (MORSE model) was developed by Luini and Capsoni $[\mathbf{6 1}]$ to predict the spatial and temporal rain intensity with integration time of 1-minute for different time scales (monthly or yearly). The model was developed using 104 statistical data series of precipitation for a long period of time, worldwide obtained in 22 countries and stored in the DBSG3 database of ITU-R.

The total amount of accumulated rain in the period of interest $(M T)$, the ratio between the convective rain $(M C)$ and the total amount of rain $\beta=M C / M T$ for a certain location are required as input parameters, they can be obtained from databases and are globally provided in Rec. ITU-R P.837-6 [57].

MORSE is founded on the following analytical expression for rainfall rate probability statistic:

$$
p(\%)=100 \cdot P_{0}\left[\ln \left(\frac{R_{a}+R_{\text {low }}}{R+R_{\text {low }}}\right)\right]^{n}
$$

where: 


$$
\begin{gathered}
n=a \cdot \beta^{b}+c \\
n=d \cdot R_{a}{ }^{e}+f \\
R_{\text {low }}=g \cdot \beta^{h}+k \\
P_{0}=\frac{M T}{\left(R_{a}+R_{\text {low }}\right) \cdot \gamma\left(n+1, \quad \ln \left(\frac{R_{a}+R_{\text {low }}}{R_{\text {low }}}\right)\right)}
\end{gathered}
$$

with:

$P_{0} \quad$ : Defines the behavior of the curve when $R$ goes to $0 \mathrm{~mm} / \mathrm{h}$.

$R_{a}$ : Asymptotic value of probability $p$ directly related with the maximum measured point rain rate.

$n$ : Mainly used to define the shape of the curve.

$R_{\text {low }}$ : Allows that the probability takes a finite value when the rainfall rate tends to $0 \mathrm{~mm} / \mathrm{h}$ (as it is physically the case).

The coefficients $a, b, c, d, e, f, g, h$ of the previous expressions depend on the time basis that is used (yearly, monthly, hourly). The annual basis equations of the model were obtained from a calibration procedure of the previous formulas by reducing the estimation error and optimizing the process with Genetic Algorithms [61].

First, the annual $M T(\mathrm{~mm})$ and $\beta$ (between 0-1) for the location (Latitude, Longitude) of interest is determined. Having $\beta$, then:

$$
\bar{\beta}=\left\{\begin{array}{cc}
\beta & \text { for } \quad \beta \geq 0.001 \\
0.001 & \text { for } \quad \beta<0.001
\end{array}\right.
$$

which is used to estimate the intermediate parameter $n$ :

$$
n=-36.1763 \bar{\beta}^{0.1242}+36.9178
$$

$R_{a}$ is computed as:

$$
R_{a}=\left(\frac{n-1.4386}{8.43 \cdot 10^{-4}}\right)^{\frac{1}{1.3531}}
$$

and:

$$
R_{\text {low }}=\left\{\begin{array}{cc}
31.8498 \bar{\beta}^{-0.0086}-31.9399 & \text { for } \quad \bar{\beta} \leq 0.72 \\
10^{-4} & \text { for } \bar{\beta}<0.72
\end{array}\right.
$$

The accumulated total local amount of rain in an hourly basis is:

$$
M T^{\prime}=\frac{M T}{365.25 \cdot 24}
$$


and the rain probability in an average year:

$$
P_{0}=\frac{M T^{\prime}}{\left(R_{a}+R_{\text {low }}\right) \cdot \gamma\left(n+1, \quad \ln \left(\frac{R_{a}+R_{\text {low }}}{R_{\text {low }}}\right)\right)}
$$

where $\gamma$ is a segment of the Gamma Function with $\gamma(a, x)=\int_{0}^{x} e^{-t} t^{a-1} d t$.

Having then:

$$
p_{0}=100 \cdot P_{0}\left[\ln \left(\frac{R_{a}+R_{\text {low }}}{0.2+R_{\text {low }}}\right)\right]^{n}
$$

with the exceedance probability $p(\%)$ of a rainfall intensity $R$ equal to:

$$
p=\left\{\begin{array}{cc}
p_{0} & R \leq 0.2 \mathrm{~mm} / \mathrm{h} \\
100 \cdot P_{0}\left[\ln \left(\frac{R_{a}+R_{\text {low }}}{R+R_{\text {low }}}\right)\right]^{n} & 0.2 \mathrm{~mm} / \mathrm{h} \leq R \leq\left(R_{a}-1\right) \mathrm{mm} / \mathrm{h} \\
0 & R>\left(R_{a}-1\right) \mathrm{mm} / \mathrm{h}
\end{array}\right.
$$

\subsubsection{ONERA Model (Annex 1 of ITU-R Rec. P.837-7)}

The model developed by ONERA (Office National d'Etudes et Recherches Aérospatiales) is an improvement proposed [62] to the ITU-R Rec. P.837 model that has been recently approved and adopted in Rec. ITU-R P.837-7 [58]. Its main feature is that it takes into account the monthly variability of rain and temperature obtained from databases (although local values can be used either).

The prediction method calculates the rainfall rate that is exceeded for a certain outage probability, using the total rainfall and the average surface temperature for each month of the year. Digital maps of these parameters were produced from 50 years of measurements collected by the Global Prediction Climatology Centre (GPCC) and from 36 years of measurements of the ECMWF ERA Interim. The model improves the overall accuracy of the previous ITU-R Recommendation version for low probability levels and in coastal and sea regions.

For using the method, the rainfall rate exceedance probability $p(\%)$ and the location latitude (Lat) and longitude (Lon) (both in degrees) must be established. Values for these input parameters are provided in tables with a resolution of $0.25^{\circ}$ in latitude and longitude.

For each month of the year, the number of the month $(i i)$ and the amount of days per month $\left(N_{i i}\right)$ are defined as:

\begin{tabular}{ccccccccccccc}
\hline \hline Month & Jan & Feb & Mar & Apr & May & Jun & Jul & Aug & Sep & Oct & Nov & Dec \\
\hline$i i$ & 01 & 02 & 03 & 04 & 05 & 06 & 07 & 08 & 09 & 10 & 11 & 12 \\
$N_{i i}$ & 31 & 28.25 & 31 & 30 & 31 & 30 & 31 & 31 & 30 & 31 & 30 & 31 \\
\hline \hline
\end{tabular}


The average monthly surface temperature $T_{i i}(K)$ is determined for the given location (Lat, Lon) for each month number $i i$ (with $i i$ from 01 to 12), from long-term reliable data.

If there are not reliable long-term local data for the exact location, the monthly mean surface temperatures $T_{i i}(\mathrm{~K})$ can be obtained from the digital maps of Rec. ITU-R P.1510-1 [28] or by performing the bilinear interpolation method of Rec. ITU-R P.1144-7 to each temperature $T_{i i}$ (which must be later converted from $\mathrm{K}$ to ${ }^{\circ} \mathrm{C}$ ).

For each month number $i$, the monthly mean total rainfall $M T_{i i}(\mathrm{~mm})$ is determined for the location, and if there are not exact data available the bilinear interpolation is applied as in the previous step.

The parameter $r_{i i}$, which relates the average surface temperature with the average rainfall rate for each month, is computed as:

$$
r_{i i}=\left\{\begin{array}{ccc}
0.5874 e^{0.0883 T_{i i}} & \text { for } & T_{i i} \geq 0{ }^{\circ} \mathrm{C} \\
0.5874 & \text { for } T_{i i}<0^{\circ} \mathrm{C}
\end{array} \quad[\mathrm{mm} / \mathrm{h}]\right.
$$

The $P_{0}$ values for each month are:

$$
P_{0_{i i}}=100 \frac{M T_{i i}}{24 \cdot N_{i i} \cdot r_{i i}} \quad[\%]
$$

If $P_{0_{i i}}>70$, set $P_{0_{i i}}=70$ and $r_{i i}=\frac{100}{70} \frac{M T_{i i}}{24 \cdot N_{i i}}$.

The annual probability of rain $P_{0_{\text {annual }}}=P(R>0)$ is:

$$
P_{0_{\text {annual }}}=\frac{\sum_{i i=1}^{12} N_{i i} \cdot P_{0_{i i}}}{365.25} \quad[\%]
$$

At the end of the procedure, if the desired rainfall rate probability of exceedance $p$ is greater than $P_{0_{\text {annual }}}$, the rainfall rate for this probability is $0 \mathrm{~mm} / \mathrm{h}$.

If the desired rainfall rate probability of exceedance $p$, is less than or equal to $P_{0_{\text {annual }}}$, the rainfall rate $R_{r e f}$ is adjusted until the absolute value of the relative error between the annual rainfall rate probability of exceedance $P\left(R>R_{\text {ref }}\right)$ and the desired rainfall rate probability of exceedance $p$ is less than $0.001 \%$. That is:

$$
\left|\frac{P\left(R>R_{r e f}\right)}{p}-1\right|<0.001
$$

where:

$$
\begin{gathered}
P\left(R>R_{r e f}\right)=\frac{\sum_{i i}^{12} N_{i i} \cdot P_{i i}\left(R>R_{r e f}\right)}{365.25} \quad[\%] \\
P_{i i}\left(R>R_{r e f}\right)=P_{0_{i i}} \cdot Q\left[\frac{\ln \left(R_{r e f}\right)+0.7938-\ln \left(r_{i i}\right)}{1.26}\right]
\end{gathered}
$$

and: 


$$
Q(x)=\frac{1}{\sqrt{2 \pi}} \int_{x}^{\infty} e^{-\frac{t^{2}}{2}} d t
$$

At the end of the adjustment process, set $R_{p}=R_{\text {ref }}$.

A MATLAB code that implements this method is available in the ITU-R web site.

\subsubsection{Rain attenuation prediction models}

There are several models developed in the last decades to predict rain attenuation in slant paths $[\mathbf{6 3}]$; they can be grouped in two types:

- Physical models. They extract parameters used to describe and model the physical basis of the phenomenon (such as rain height, the horizontal and vertical reduction factors and the rainfall rate distribution in a rain cell) from meteorological radar measurements.

- Semi-empirical models. They are developed by an adjustment of the experimental attenuation measurements in several locations, frequencies and elevation angles, and require statistical parameters such as the effective length of the path or the horizontal and vertical reduction factors. They also have an underlying physical basis.

Although it may seem that physical models should be more accurate for attenuation predictions, semi-empirical models are more convenient due to the difficulty of collecting all the necessary parameters for obtaining accurate results with the physical models.

The rain specific attenuation is a very important parameter defined as the attenuation that is reached in a certain medium per distance unit, usually expressed in $\mathrm{dB} / \mathrm{km}$. An equation to estimate it - that has its physical basis in equation (2.1) - is given in Rec. ITUR P.838-3 [64], the specific attenuation being related to the rainfall rate $R$ by means of a power-law:

$$
\gamma_{R}=k \cdot R^{\alpha} \quad(\mathrm{dB} / \mathrm{km})
$$

The coefficients $k$ and $\alpha$ depend on frequency and polarization, respectively; and are determined by equations given in the above mentioned ITU-R Recommendation.

The main prediction methods of rain attenuation are compared in Table 2.2. To do so, it is convenient to have into account their main characteristics, the basis to describe the spatial non-uniformity of rain and input parameters, as was done in [19] and [65]. 


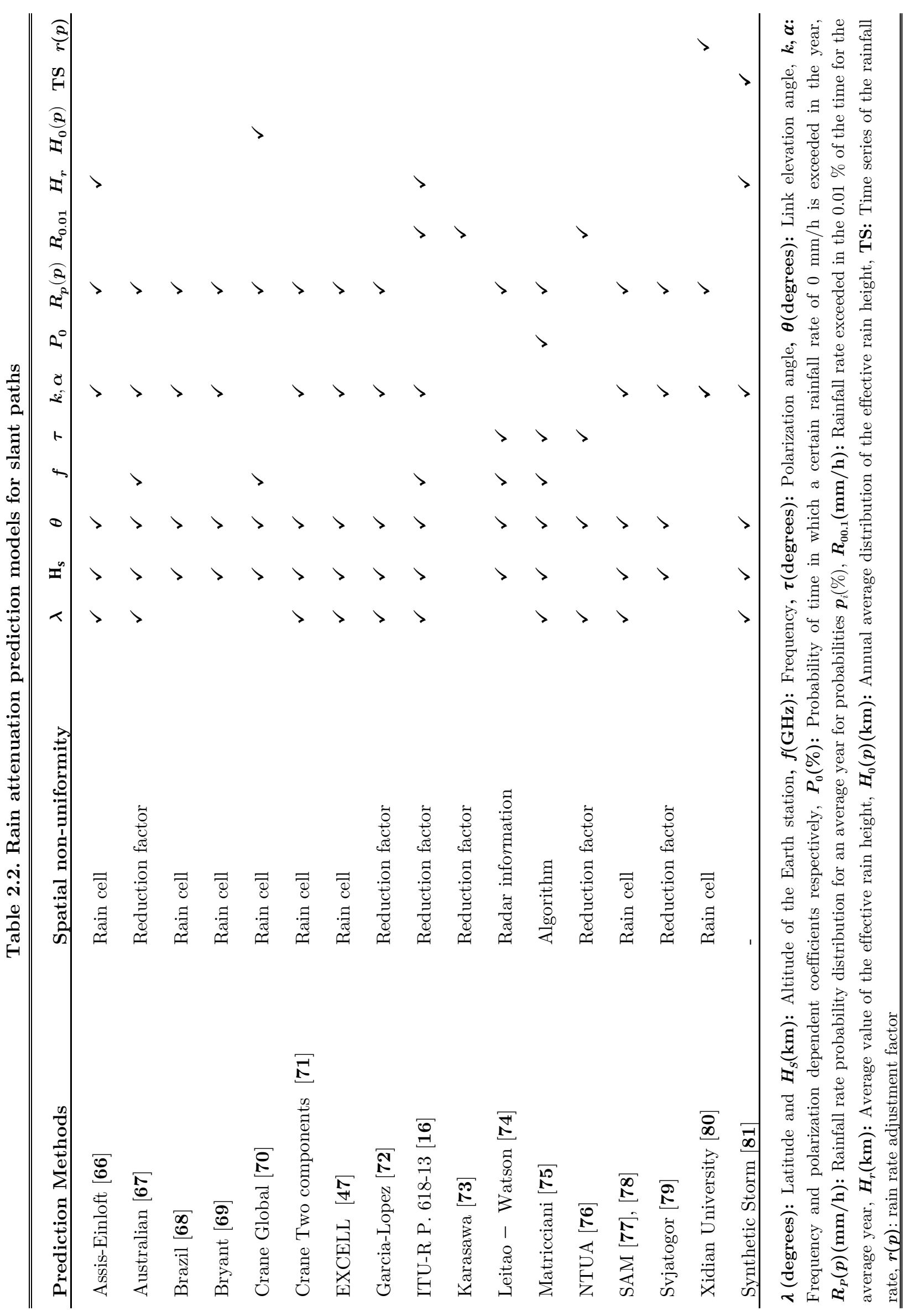


It is convenient to provide a brief explanation of the classifications given in the "spatial non-uniformity" column of Table 2.2. "Radar information" means that the spatial nonuniformity was determined by using information of radar measurements; the same applies for algorithm (the cited references can be consulted for more detailed information about the involved algorithms). On the other hand, "rain cell" concept means that some of the previously mentioned cell models were used. Finally, "reduction factor" (horizontal and vertical) are those obtained from the rain structure (hence, experimental campaigns were taken into account), and are considered more adequate to estimate diversity gain for sites with different rainfall profiles. The reduction factors express the ratio between the total attenuation and the attenuation that may produce a uniform rain. This approach is more accurate if the horizontal and vertical factors are given separately.

\subsection{Tropospheric scintillation}

Tropospheric scintillation is the name given to the fast fluctuations in the signal level due to small variations in the atmospheric refraction index, as the result of temporal and spatial irregularities in the composition of the atmosphere, caused by in-homogeneous values of temperature, humidity and pressure.

Fades due to tropospheric scintillation can be the major impairment for the communication availability in the absence of precipitation in systems with a low margin and a low elevation angle (below $10^{\circ}$ ) [16]. Moreover, the fast fluctuations in the receiver signal level can generate problems in the antenna tracking system, in the up-link power control, in the PIMT implementation and in the behavior of some modulations [82]. That is why the knowledge of the dynamical characteristics of scintillation can aid in the design of mitigation systems.

It has been experimentally verified that a decrease in the elevation angle or in the antenna diameter and an increase in frequency [83] produce an increase in the scintillation intensity. Moreover, scintillation intensity has high climatological dependence due to its strong relation with the probability of atmospheric turbulence formation. In temperate zones, seasonal and hourly variability are observed in the measurements as an increase of scintillation intensity in the warmer hours of the day (around noon) in the summer, due to the higher probability of convective phenomena.

Scintillation is originated by phenomena that take place above $1000 \mathrm{~m}$ of the surface level; and the measurement of meteorological variables in that zone is cumbersome. That is the main reason to correlate scintillation with meteorological parameters at surface level, easier to measure but less correlated with the real behavior at such higher layers of the troposphere. The meteorological parameters at surface level such as temperature - a surface warming produces a great exchange of energy [84] - wind velocity and humidity have a high influence in the turbulence formation. This explains the variability of scintillation intensity in time, following the changes of meteorological parameters.

Among the surface variables, the most correlated one with scintillation is the wet part of the refraction index, $N_{\text {wet }}$, which depends on temperature and absolute humidity, although its bad correlation with the scintillation diurnal variability was proven in [85]. Another term 
influencing in scintillation is the amount of clouds in the propagation path, since they act as a turbulence generator, especially clouds with high liquid water content.

Tropospheric scintillation is formally divided in "wet scintillation" (when it occurs in the presence of rain) and "dry scintillation" (when it is measured in clear sky condition or without rain).

\subsubsection{Tropospheric scintillation prediction models}

Most of the scintillation models are semi-empirical, and are based on the Tatarskii theory [86] and in the variance and amplitude distributions of Moulsley and Vilar [87] adjusted with experimental measurements. Only the model of the Université Catholique de Louvain (UCLouvain) [88] can be considered as physical because it is based on the estimation of the refractive index structure constant and requires local vertical profiles.

The other models basically have been developed by correlating meteorological variables at surface level with experimental measurements of scintillation, since radio-sounding measurements have a relatively low availability at global scale. The three most used parameters are: the temperature, the humidity (absolute or relative) and the wet part of the refraction index.

A comparative between the most used models to predict scintillation in clear sky condition is shown in Table 2.3, specifying the main parameters in each one. It is worth mentioning that the model of Rec. ITU-R P.618-13 is an improvement to the Karasawa model, from which the equation to obtain the aperture antenna approximation factor is taken.

Most of the models propose equations obtained by regressions of empirical data from several propagation experiments. 


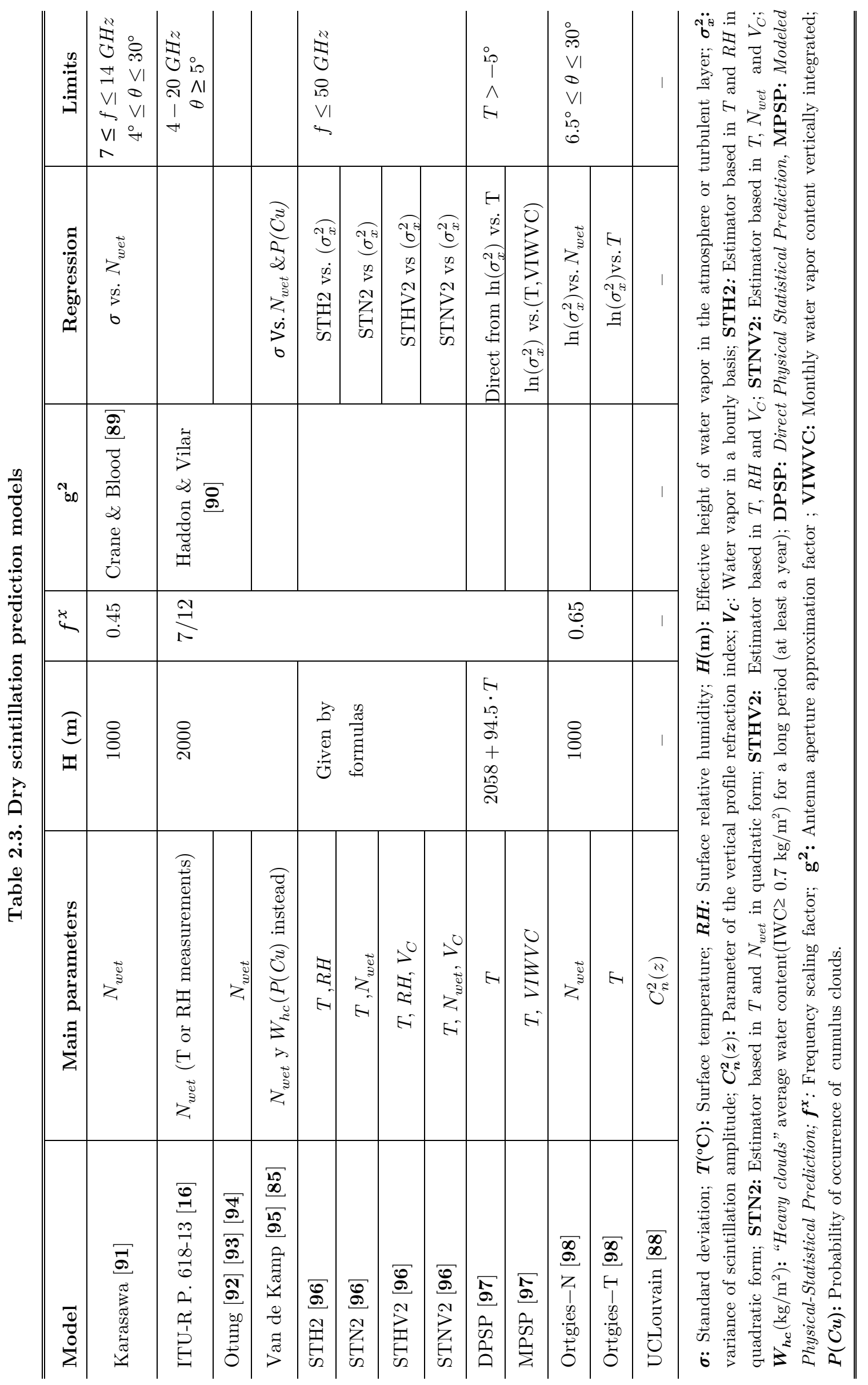


An important work was done by Matricciani and Riva [99] in order to characterize wet scintillation. They proposed an equation derived from experimental results in two links from different regions of Europe at $18.7 \mathrm{GHz}$ that relates rain attenuation with scintillation. In that study, the average rain attenuation $A(\mathrm{~dB})$ and the average standard deviation $\sigma_{m}(\mathrm{~dB})$ in 1-minute intervals of $\sigma(\mathrm{dB})$ are related by a power-law and a constant:

$$
\sigma_{m}=C_{3 a} A^{\frac{5}{12}}
$$

If scintillation is a consequence of a high and thin turbulent layer, then:

$$
\sigma_{m}=C_{3 b} A^{\frac{5}{12 \cdot 0.9}}
$$

where $C_{3 a, 3 b}$ are constants that can be estimated by applying the scintillation formulas given in Rec. ITU-R P.618-13 [16]. For a more conservative design, a fitting procedure of the conditional standard deviation $\sigma$ depending of $A$ is also proposed:

$$
\sigma_{s}=C_{s} A^{d}
$$

where $C_{s}$ and $d$ are the parameters to optimize.

Although the model has not been tested worldwide, a study carried out in the UPM with a $50 \mathrm{GHz}$ beacon signal $[\mathbf{1 0 0}]$ reports good agreement with the proposed formulas, particularly for attenuation in the $1-10 \mathrm{~dB}$ range.

\subsection{Fade dynamics}

A key parameter used to characterize the signal dynamics is fade, as the time in which the signal intensity exceeds (falls below) a certain attenuation (or power) threshold.

Some of the fade dynamics concepts are [101]:

1. Fade duration, as the time between two consecutive crossings of the received signal value above a certain attenuation threshold $A_{t h}$.

2. Inter-fade duration, as the time interval between two crossings below a certain attenuation threshold $A_{t h}$.

3. Fade slope, defined as the change rate of attenuation with time (in $\mathrm{dB} / \mathrm{s}$ ).

4. Inter-event interval, as the time between two consecutive attenuation events clearly identified.

They can be identified in the attenuation time series of Figure 2.2. 


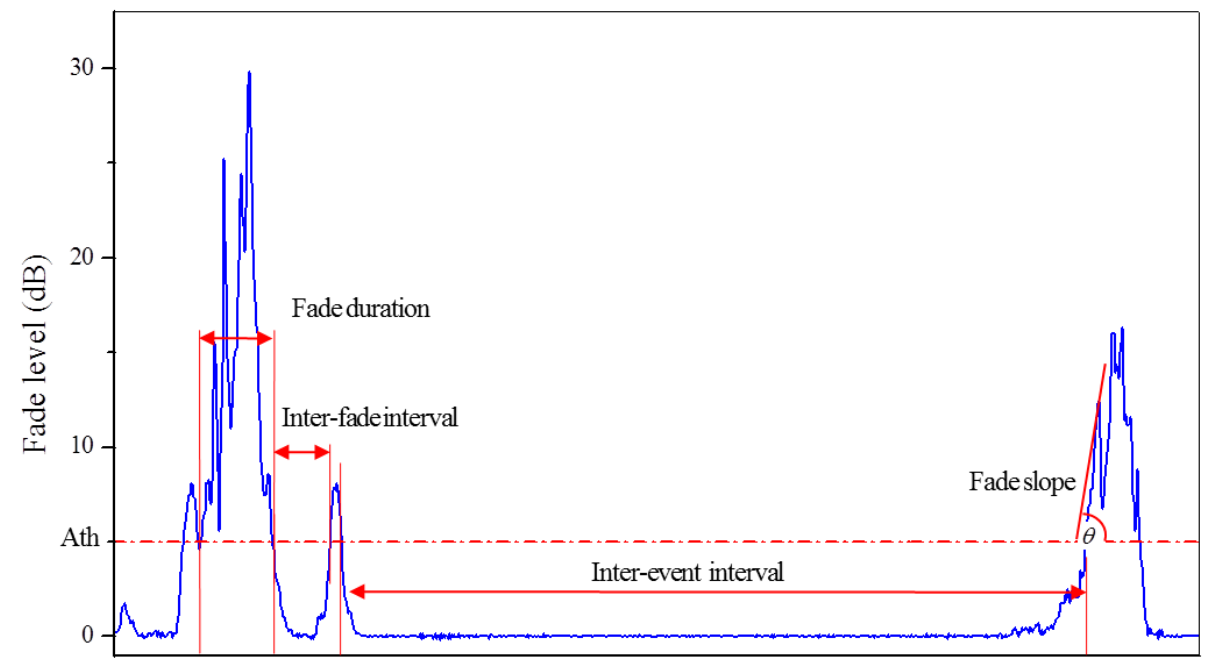

Time

Figure 2.2. Dynamic characteristics of an attenuation time series.

The statistics of these parameters are also known as "second-order statistics" of the propagation channel, and they are usually related to the system flexibility (the system characteristic that allows it adapting when the propagation conditions change, using some PIMT). Therefore, fade dynamics is crucial in the design of PIMT. Moreover, the spectral characteristics are also necessary to filter out the fast signal fluctuations due to scintillation.

The knowledge of system fade dynamics allows several operative actions based on:

- System outage: Fade duration gives information about outage periods in the link due to propagation effects.

- PIMT: Estimation of the adequate statistical duration in which the mitigation technique must act before the system returns to a normal behavior.

- System resource sharing: It is important to know the outage statistics from an operator point of view and, based on that, to relocate the system resources to other users.

- System waveform: It is a key parameter in the selection process of the better FEC (Forward Error Correction) and modulation techniques. In satellite systems, the propagation channel produces burst errors rather than independent errors.

Besides these, other interesting aspects are: frequency scaling, used to estimate the magnitude of a certain propagation effect (attenuation, scintillation, depolarization, etc.) at a frequency from the knowledge of the behavior of this variable at another frequency - usually a lower one - and the spatial and temporal correlation (among a wide range of time and distance) of attenuation.

\subsubsection{Fade duration modeling}

One of the most used parameters in fade dynamics is fade duration $(D)$. Several models developed from experimental data have been proposed to describe fade duration behavior as a combination of statistical distributions such as exponential, power-law and log-normal 
functions. Four cumulative distribution functions are used to describe the behavior of this variable $[\mathbf{1 0 1}]$ :

1. $P\left(d>D \mid a>A_{t h}\right)$, the probability of occurrence of fades with duration $d$ longer than $D$ in seconds (s), given that the attenuation $a$ is greater than $A_{t h}(\mathrm{~dB})$. This can be estimated from the ratio of the number of fades of durations longer than $D$ to the total number of fades observed, given that the threshold $A_{t h}$ is exceeded.

2. $F\left(d>D \mid a>A_{t h}\right)$, the total fraction of fade time (between 0 and 1) due to fades of duration $d$ longer than $D$, given that the attenuation $a$ is greater than $A_{t h}(\mathrm{~dB})$. This can be estimated from the ratio of the total fading due to fades of duration longer than $D$ given that the threshold $A_{t h}$ is exceeded, to the total exceedance time of the threshold.

3. $N\left(d>D, a>A_{t h}\right)$, the total number of fade events of duration $d$ longer than $D$ for a given attenuation threshold $A_{t h}$.

4. $T\left(d>D \mid a>A_{t h}\right)$, the total fading time due to fades of duration $d$ longer than $\mathrm{D}$ for the attenuation threshold $A_{t h}$.

Hereinafter, the conditional terms of these distributions will be simplified for notational convenience, because the thresholds are known in each case. Then, $P\left(d>D \mid a>A_{t h}\right)$ is represented as $P(D)$, and the same applies for $F(D), N(D)$ and $T(D)$.

Since COST 205 [102], the standard way to model fade durations is by considering two time scales: the fades of long durations (usually longer than 30 seconds) produced by rain and the fades with short durations mostly produced by scintillation. Since then, fade duration distributions are modeled with a combination of two probability distributions, one related to short fade durations and the other to long fades.

Several studies on fade durations have been carried out in different geographical areas and conditions; among them the following ones can be found:

In [103] Mandeep presented a fade duration result analysis from a natural year (2007) data of an un-filtered Ku beacon signal in Malaysia, at the Equatorial region.

In [104] Cheffena and Amaya used a large amount of data from several regions and frequencies - although data from Ka-band were the most abundant- and also proposed a model - known as the CRC (Communication Research Centre Canada) model - for the fade duration probability of occurrence by using a combination of two log-normal functions, one used to describe the distribution of short fades and the other for the long fades. In a previous work [105], Amaya and Nguyen compared the experimental CCDFs of the number of fades obtained from a $20 \mathrm{GHz}$ unfiltered beacon receiver in a 4-year period (2006-2009) at the CRC, Ottawa, with several models (CRC, ITU-R, D-H y COST 205).

The distributions obtained for fade and inter-fade durations for a natural year (2000) with a $50 \mathrm{GHz}$ un-filtered beacon in Madrid, Spain are presented in [106]. In this work the authors have proved that a power-law function can model accurately short fade and interfade durations, and that medium and long fade durations can be modeled with a log-normal distribution. 
The most relevant fade duration models found in the references are summarized in Table 2.4 .

Table 2.4. Fade duration prediction models

\begin{tabular}{lll}
\hline \hline Prediction models & Characteristics & Input data \\
\hline USA model (also known as & Short and long fades are modeled & \\
Dissanayake-Haidara, D-H $)^{2}[\mathbf{1 0 7}]$ & with a log-normal function for & \\
CRC model $[\mathbf{1 0 4}]$ & each one. & \\
\hline COST 205 $[\mathbf{1 0 2}]$ & Use of a power-law function to & Frequency. \\
Paraboni-Riva $[\mathbf{1 0 8}]$ & model short durations and a log- & - Attenuation \\
ITU-R P. 1623-1 $[\mathbf{1 0 1}]$ & normal function to model long & threshold. \\
& durations. & Elevation angle. \\
\hline Bråten model $[\mathbf{1 0 9}]$ & Use of three decreasing & \\
Lekkla model $[\mathbf{1 1 0}]$ & exponential functions. & \\
& Use of two decreasing exponential & \\
\hline \hline
\end{tabular}

Most of the models of Table 2.4 use a log-normal distribution to represent the long fade duration part of the total distributions. On the other hand, to model the part of the distributions related with the short fades - mostly produced by scintillation - several statistical functions such as the power-law, the exponential and the log-normal have been used. In the case of the ITU-R and USA models, they also include a procedure to obtain the $F(D)$ distribution.

The Bråten and Lekkta models use exponential functions to represent either the short or the long duration distributions. The Lekkta model will not be addressed because it is based on radiometric measurements (that do not detect scintillation) and its performance for unfiltered beacon data, the type of data used in this work, is poor [111]. Moreover, the Rec. ITU-R P. 1623-1 model is based on the Paraboni-Riva model [108] (although with some modifications), a reason for not using the latter in future comparisons.

Having into account the previous considerations, it can be concluded that the description of the fade duration distributions can be accomplished by using a two-distribution combination, being possible to express the probability of occurrence in this case as:

$$
P(D)=\alpha_{n} P_{S}(D)+\left(1-\alpha_{n}\right) P_{L}(D)
$$

where $\alpha_{n}$ is the conditional probability of occurrence associated to the short fade duration distribution $P_{S}(D)$, given for an attenuation threshold $A_{t h}$ (in $\mathrm{dB}$ ), and $\left(1-\alpha_{n}\right)$ is the probability of occurrence associated to longer durations $P_{L}(D)$. The limit between short and long durations varies from one model to other.

${ }^{2}$ A model presented by the United States of America (USA) toward the revision of the Rec. ITUR P.1623 prediction model, usually referenced by the name of its authors A. Dissanayake and F. Haidara (D-H). 
In an analogous way, the total fraction of fade time distribution can be defined as:

$$
F(D)=\alpha_{t} F_{S}(D)+\left(1-\alpha_{t}\right) F_{L}(D)
$$

where $\alpha_{t}$ is the conditional fraction of time associated with the short duration composite fading time distribution $F_{S}(D)$ and $\left(1-\alpha_{t}\right)$ is the fraction of time associated to the long duration distribution $F_{L}(D)$, both for certain $A_{t h}$.

The log-normal distribution that describes the probability of long fade durations has mean $\mu_{n, 2}$ and standard deviation $\sigma_{n, 2}$, with the conditional CCDF (in the case of the probability of occurrence) being:

$$
P_{L}(D)=\frac{\operatorname{erfc}\left(\frac{\ln D-\mu_{n, 2}}{\sigma_{n, 2} \sqrt{2}}\right)}{\operatorname{erfc}\left(\frac{\ln D_{0}-\mu_{n, 2}}{\sigma_{n, 2} \sqrt{2}}\right)}
$$

with $\operatorname{erfc}(x)=\frac{2}{\sqrt{\pi}} \int_{x}^{+\infty} \exp \left(-t^{2}\right) d t$. The same function can be used for $F_{L}(D)$.

The minimum fade duration, $D_{0}$, is equal to the receiver time resolution when it is derived from attenuation measurements.

Short fade durations, $P_{S}(D)$ (or $F_{S}(D)$ in its case) can be modeled with the following functions:

1. Exponential: $P_{S}(D)=e^{E_{n} D_{0}} e^{-E_{n} D}$ with only the $E_{n}$ parameter.

2. Power-law: $P_{S}(D)=D_{0}{ }^{B_{n}} D^{-B_{n}}$, with only the $B_{n}$ parameter.

3. Log-normal: $\quad P_{S}(D)=\frac{\operatorname{erfc}\left(\frac{\ln D-\mu_{n, 1}}{\sigma_{n, 1} \sqrt{2}}\right)}{\operatorname{erfc}\left(\frac{\ln D_{0}-\mu_{n, 1}}{\sigma_{n, 1} \sqrt{2}}\right)}$; where $\mu_{n, 1}$ and $\sigma_{n, 1}$ are the distribution mean and standard deviation, respectively.

Instead of defining independently $P(D)$ and $F(D)$, a different approach can be used for obtaining the fading time distribution $F(D)$ from $P(D)$. This takes advantage of the fact that the different cumulative functions used to characterize fade durations are related to each other. Then, the absolute number of fades $N(D)=N_{\text {tot }} \cdot P(D)$, with $N_{\text {tot }}$ being the total number of fades, is related to the total fading time $T(D)=T_{\text {tot }} \cdot F(D)$, with $T_{\text {tot }}$ being the total time of fades, through (2.25) [108], for each attenuation threshold:

$$
\begin{aligned}
& \frac{d N(D)}{d D} D=\frac{d T(D)}{d D} \\
& \frac{d N(D)}{d(\ln D)} D=\frac{d T(D)}{d(\ln D)}
\end{aligned}
$$

The model that uses three cascade exponentials - commonly known as Bråten modelwas proposed only for the probability of occurrence distribution. It is the result of a study carried out by Telenor (Norway) and the CRC (Canada) [109]. It is based on ACTS 
(Advanced Communications Technology Satellite) measurements for beacons at $20.2 \mathrm{GHz}$ and $27.5 \mathrm{GHz}$. The equation that describes the probability distribution for a given attenuation is:

$$
P(D)=\alpha_{1} e^{-\frac{D}{\beta_{1}}}+\alpha_{2} e^{-\frac{D}{\beta_{2}}}+\alpha_{3} e^{-\frac{D}{\beta_{3}}}
$$

Each term in the sum is related to a specific fade origin: the first term describes the fades of very short duration due to tropospheric scintillation, the second describes the short fade durations produced by convective rain and the third one describes the long fade durations related to stratiform rain.

The positive variables $\alpha_{i}$ represent the conditional probability of occurrence of the different types of phenomena in the conditional distribution; being necessary that $\sum \alpha_{i}=1$; then, the total probability is the sum of all the probabilities corresponding for each phenomenon. If this model is compared with others that start at 1-s duration (meaning that durations shorter than this value are not considered and the total fading time is composed only by fades with durations equal to or longer than 1-s), it can be concluded that this method is normalized to $0 \mathrm{~s}$ (the total fading time corresponds to fade durations equal to or longer than $0 \mathrm{~s}$ ) because the total fading time is fulfilled only when $D=0$. In that way, this method relates the probability of occurrence of fades of any duration.

The positive variables $\beta_{i}$ are called characteristic durations, they are given in seconds and represent the average fade duration value. These values have a physical interpretation: $\beta_{1}$ will be the characteristic duration of very short fades related to scintillation, $\beta_{2}$ represents the duration of short fades produced by convective rains and $\beta_{3}$ represents the duration of long fades produced by stratiform rain.

Several equations dependent on climatological parameters and other values are provided to adjust $\alpha_{i}$ and $\beta_{i}$ by means of linear regression techniques. For other conditions these values can suffer some changes. As the model was derived from Ka-band measurement data sets, its estimates for higher frequencies may be questioned. Moreover, some specific combinations of singular input values can produce odd and unrealistic results.

For all the distribution combinations, the coefficient values that best fit a particular case can be obtained from experimental data, and then a comparison between all function combinations can be carried out. To obtain these coefficients some fitting procedures are available, such as the following:

\section{ONERA fitting procedure}

In the scope of an ESA (European Space Agency) project, ONERA proposed a procedure [112] for determining the coefficients to be used for three possible combinations of short- and long-duration distributions (i.e. log-normal with log-normal, exponential with log-normal, power-law with log-normal). Here the procedure is explained only for the $P(D)$ case, but it can also be applied for $F(D)$.

This fitting is based on minimizing in the first place the error $e$ between the predicted long fade distribution function $P_{L}(D)$ (i.e. $P_{L}\left(D_{i} \mid D_{i} \geq D_{\text {min }}\right)$ ) and the long fade part of the experimental CCDF of the probability of occurrence $P_{\text {exp }}(D)$ (i.e. $P_{\text {exp }}\left(D_{i} \mid D_{i} \geq D_{\min }\right)$ ) for a certain location and attenuation threshold: 


$$
e=\sum_{D_{i}}^{D_{\max }}\left|\frac{\log \left[P_{L}\left(D_{i} \mid D_{i} \geq D_{\min }\right)\right]-\log \left[P_{e x p}\left(D_{i} \mid D_{i} \geq D_{\min }\right)\right]}{\log \left[P_{\text {exp }}\left(D_{i} \mid D_{i} \geq D_{\min }\right)\right]}\right| \quad \text { for } D \geq D_{\text {min }}
$$

where $D_{\text {min }}=60 \mathrm{~s}$ is the limit between short and long fades and is assumed in order to discard the contributions of fade durations shorter than this value (related with scintillation and fast fluctuations due to rain) to the experimental distribution, and $D_{\max }$ is the maximum available value of fade durations in the data. The variable parameters used for minimizing $e$ are $\mu_{n, 2}$ and $\sigma_{n, 2}$ of $P_{L}(D)$, then, their optimized values are obtained from this first step.

Second, taking the durations associated to $P_{S}\left(D_{i} \mid D_{i} \geq D_{0}\right)$ as negligible for the experimental distribution associated to the long durations $P_{\text {exp }}\left(D_{i} \mid D_{i} \geq D_{\text {min }}\right)$, the probability of occurrence $\left(1-\alpha_{n}\right)$ associated to $P_{L}(D)$ is obtained as follows:

$$
\begin{gathered}
P_{\text {exp }}\left(D_{i} \mid D_{i} \geq D_{0}\right)=\alpha_{n, i} P_{S}\left(D_{i} \mid D_{i} \geq D_{0}\right)+\left(1-\alpha_{n, i}\right) P_{L}\left(D_{i} \mid D_{i} \geq D_{0}\right) \\
P_{e x p}\left(D_{i} \mid D_{i} \geq D_{\text {min }}\right) \approx\left(1-\alpha_{n, i}\right) P_{L}\left(D_{i} \mid D_{i} \geq D_{\text {min }}\right) \\
\alpha_{n, i}=1-\frac{P_{\text {exp }}\left(D_{i} \mid D_{i} \geq D_{\min }\right)}{P_{L}\left(D_{i} \mid D_{i} \geq D_{\min }\right)} \\
\text { for } D_{\min } \leq D_{i} \leq D_{\max }
\end{gathered}
$$

where the optimized $\mu_{n, 2}$ and $\sigma_{n, 2}$ are now used in $P_{L}\left(D_{i} \mid D_{i} \geq D_{\min }\right)$ for deriving $\alpha_{n, i}$. From (2.37), $\alpha_{n}$ is obtained as the mean of $\alpha_{n, i}$.

Moreover, a $P_{S}\left(D_{i} \mid D_{i} \geq D_{0}\right)$ distribution is obtained from (2.36) using now $\alpha_{n}$ instead of $\alpha_{n, i}$ and using $P_{L}\left(D_{i} \mid D_{i} \geq D_{0}\right)$ with the optimized $\mu_{n, 2}$ and $\sigma_{n, 2}$ values.

Finally, the optimized parameters of $P_{S}\left(D_{i} \mid D_{i} \leq D_{\text {min }}\right)$ are obtained by minimizing (2.35), using now $P_{S}\left(D_{i} \mid D_{i} \leq D_{\text {min }}\right)$ instead of $P_{L}\left(D_{i} \mid D_{i} \geq D_{\text {min }}\right)$ and the previously obtained $P_{S}\left(D_{i} \mid D_{i} \geq D_{0}\right)$ distribution instead of $P_{\text {exp }}\left(D_{i} \mid D_{i} \geq D_{\text {min }}\right)$ but now limiting both to the $D_{0} \leq D_{i} \leq D_{\min }$ range. In that way, the optimized parameters of $P_{S}(D)$ (i.e. $\mu_{n, 1}, \sigma_{n, 1}$, $E_{n}, B_{n}$ depending the case) are derived. Now, the predicted $P(D)$ of $(2.22)$ is obtained with the $P_{S}(D)$ and $P_{L}(D)$ parameters as well as with $\alpha_{n}$.

\section{Bråten fitting procedure}

This procedure is used in the case of the three negative exponentials model of (2.34). It adjusts the data by minimizing the error function of (2.38) for all $D_{i}$ :

$$
\varepsilon_{i}(D)=\log \left[\frac{P_{e x p}\left(D_{i} \mid D_{i} \geq D_{0}\right)}{P\left(D_{i} \mid D_{i} \geq D_{0}\right)}\right]
$$

where $i=1,2,3 \ldots$ represents the duration of fades.

Special care must be taken in the fitting due to restrictions that are imposed to the positive parameters $\alpha_{i}$ and $\beta_{i}$.

\subsubsection{Inter-fade duration modeling}

Inter-fade duration is an important parameter in aiding the design and implementation of PIMT. From an operator point of view, it is helpful to predict the expected time that a certain availability condition will last in a satellite link before another fade event takes place. 
This knowledge allows a non-uniform power distribution in the downlink; thus a higher amount of resources can be re-assigned to geographical areas where propagation conditions are more severe.

In spite of its importance, there are no physical models that describe inter-fade duration in depth, due to the complexity of its propagation phenomena mechanism. Inter-fade intervals can last from few seconds up to several months; then different inter-fade duration ranges are conditioned by different meteorological characteristics.

A prediction model for inter-fade durations was proposed by the USA to the ITU-R Study Group $3[\mathbf{1 1 3}]$. It is based on beacon measurements of the ACTS campaign and proposes an inter-fade duration CCDF with three log-normal terms. Each of the three lognormal terms of the inter-fade duration distribution is related to a possible origin: short durations are ruled by scintillation, medium durations -inter-fade intervals that occur within a fade event - are due to rain, and long durations occur between different rain events. The model can be used for latitudes from $0^{\circ}$ to $90^{\circ}$, elevation angle from $1^{\circ}$ to $90^{\circ}$ and frequencies between 3 and $50 \mathrm{GHz}$.

The triple log-normal distribution is:

$$
\begin{aligned}
P_{n i}\left(d>D \mid a<A_{t h}\right) & =\frac{n_{s i}}{n_{s i}+n_{r i}+n_{e i}} \frac{Q\left(\frac{\ln \left(D / D_{1 s i}\right)}{\sigma_{s i}}\right)}{Q\left(\frac{\ln \left(1 / D_{1 s i}\right)}{\sigma_{s i}}\right)}+ \\
& +\frac{n_{r i}}{n_{s i}+n_{r i}+n_{e i}} \frac{Q\left(\frac{\ln \left(D / D_{1 r i}\right)}{\sigma_{r i}}\right)}{Q\left(\frac{\ln \left(1 / D_{1 r i}\right)}{\sigma_{r i}}\right)}+\frac{n_{e i}}{n_{s i}+n_{r i}+n_{e i}} \frac{Q\left(\frac{\ln \left(D / D_{1 e i}\right)}{\sigma_{e i}}\right)}{Q\left(\frac{\ln \left(1 / D_{1 e i}\right)}{\sigma_{e i}}\right)}
\end{aligned}
$$

where:

$$
\begin{gathered}
n_{s i}=k e^{-\ln D_{1 s i}+\frac{\sigma_{s i}^{2}}{2}} \cdot \frac{Q\left(\frac{\ln \left(1 / D_{1 s i}\right)}{\sigma_{s i}}\right)}{Q\left(\frac{\ln \left(1 / D_{0 s i}\right)}{\sigma_{s i}}\right)} \\
n_{r i}=(1-k) e^{-\ln D_{1 r i}+\frac{\sigma_{r i}^{2}}{2}} \cdot \frac{Q\left(\frac{\ln \left(1 / D_{1 r i}\right)}{\sigma_{r i}}\right)}{Q\left(\frac{\ln \left(1 / D_{0 r i}\right)}{\sigma_{r i}}\right)} \\
n_{e i}=(1-k) e^{-\ln D_{1 e i}+\frac{\sigma_{e i}^{2}}{2}} \cdot \frac{Q\left(\frac{\ln \left(1 / D_{1 e i}\right)}{\sigma_{e i}}\right)}{Q\left(\frac{\ln \left(1 / D_{0 e i}\right)}{\sigma_{e i}}\right)}
\end{gathered}
$$

with $k$ and $(1-k)$ being the fractions of time associated with scintillation and rain fades respectively, for a certain attenuation threshold $A_{t h}(\mathrm{~dB})$.

The variables $n_{s i}, \quad n_{r i}$ and $n_{e i}$ correspond to a specific inter-fade duration origin: scintillation, rain (that originates intra-event durations) and time between rain events (interevent durations) respectively. The variables $\sigma$ and $D_{1}$ are the canonical parameters of each log-normal distribution. Then, for example, $\sigma_{s i}$ is the standard deviation of the scintillation- 
related inter-fade events and $D_{1 e i}$ is the mean of durations that occur between different rain events. The model also uses parameters of the USA fade duration model [107].

Besides this model, there is another modeling effort presented in [114] as a result of a study carried out by the Instituto de Telecomunicações of Aveiro, Portugal, in collaboration with the Politecnico di Milano, Italy. In this work, inter-fade durations are related with meteorological phenomena as follows:

- Small scale inter-fades (less than approximately $8 \mathrm{~s}$ ), are produced by the propagation medium microstructure non-homogeneities.

- Inter-fade durations (between 8 and $740 \mathrm{~s}$ ), are the periods in which fade events of a certain deep do not occur within a longer fade event and they depend on the rainfall rate distribution.

- Inter-event durations, are periods in which fade events of a certain depth do not occur between longer fade events easily identified in time, and are dependent on the rain cell size and velocity.

- Climatically induced durations, caused by climatological changes throughout the years.

Moreover, the first step for a future model development was taken in this study, as it is the adjustment of certain mathematical distributions (exponential, power-law and lognormal) to experimental data for the aforementioned classifications. As results, it is concluded that the power-law distribution is the best fit function for small scale and interfade duration and the log-normal distribution gives the best fit to inter-events and climatically induced durations. On the other hand, this modeling effort does not provide a function to describe the complete inter-fade duration distribution.

Until now the ITU-R does not recommend any model for inter-fade duration, this being an open research issue.

\subsubsection{Fade slope}

The characterization of fade slope can be used for predicting the channel propagation short-term behavior and designing control loops capable to follow the variations of the received signal and to decide whether a PIMT is necessary or not. A signal can have fast (mostly produced by scintillation) or slow (produced by rain and cloud attenuation) amplitude variations, the latter being the most relevant, since fast fluctuations lose the autocorrelation in few seconds [115]. The fast variations of the signal must be filtered out in order to work with the slow component; then the filter characteristics have a very high influence on the quality of the results. Also, the receiver dynamical parameters can modify the fade slope measurements. A receiver with a longer integration time reduces the instantaneous fade variation and spreads it over a longer period of time.

The fade slope probability distribution is conditioned by several climatological parameters such as the rain DSD, the rain type and the transversal wind velocity. Moreover, an increase of the propagation path distance produces a decrease in the slope value due to the smoothness effect of different rain contributions in the summation. On the other hand, an increase of the elevation angle produces an increase of the slope values. 
The fade slope expression is provided by Recommendation ITU-R P.1623-1 [101]:

$$
\zeta(t)=\frac{A\left(t+\frac{1}{2} \Delta t\right)-A\left(t-\frac{1}{2} \Delta t\right)}{\Delta t} \quad[\mathrm{~dB} / \mathrm{s}]
$$

Meaning that the fade slope $\zeta$ in a certain time $t$ is equal to the quotient between the difference of attenuations $A$ at instants $(\mathrm{t}+1 / 2 \Delta \mathrm{t})$ and $(\mathrm{t}-1 / 2 \Delta \mathrm{t})$ and the time interval $\Delta \mathrm{t}$.

The fade slope distributions have been characterized by using results of several propagation experiments in the $\mathrm{Ku}-, \mathrm{Ka}-$ and $\mathrm{V}$-band [116], [117], [118].

In spite of the availability of several studies that propose different fade slope models [117], [119], the ITU-R Rec. P. 1623-1 prediction method [101] —based on a Van de Kamp proposal $[\mathbf{1 2 0}],[\mathbf{1 2 1}]$ - is the most widely accepted, as the consulted literature states [118], $[\mathbf{1 2 2}]$, [123]. The model starts from the fade slope definition of (2.41) for filtered data and is valid for the following parameter ranges:

- Frequency between 10 and $30 \mathrm{GHz}$.

- Elevation angle between $10^{\circ}$ and $50^{\circ}$.

Also, it needs as input parameters:

$A$ : The attenuation level (in $\mathrm{dB}$ ), from 0 to $20 \mathrm{~dB}$.

$f_{B} \quad: 3 \mathrm{~dB}$ cut-off frequency of the low pass filter (in $\mathrm{Hz}$ ), from $0.001-1 \mathrm{~Hz}$.

$\Delta t \quad$ : Time interval used to calculate the fade slope (in s), from 2-200 s.

In the first place, the function $F$, which gives the dependency on the time interval length $\Delta t$ and the 3 -dB cut-off frequency of the low-pass filter $f_{B}$, is calculated:

$$
F\left(f_{B}, \Delta t\right)=\sqrt{\frac{2 \pi^{2}}{\left(1 / f_{B}^{b}+(2 \Delta t)^{b}\right)^{1 / b}}} \quad \text { with } b=2.3
$$

A graphic representation of this function for some $\Delta t$ is provided in Figure 2.3.

The standard deviation $\sigma_{\zeta}$ of the conditional fade slope at a given attenuation level is:

$$
\sigma_{\zeta}=s \cdot F\left(f_{B}, \Delta t\right) \cdot A \quad[\mathrm{~dB} / \mathrm{s}]
$$

where $s$ is a parameter depending on the climate and the elevation angle; an overall average value for Europe and for elevation angles between $10^{\circ}$ and $50^{\circ}$ is 0.01 . This parameter can be optimized using data measurements.

The $p(\zeta \mid A)$, the conditional PDF of fade slope $\zeta$ for a given attenuation value $A$ is:

$$
p(\zeta \mid A)=\frac{2}{\pi \cdot \sigma_{\zeta}\left(1+\left(\zeta / \sigma_{\zeta}\right)^{2}\right)^{2}}
$$

If required, the conditional probability $(\mathrm{CCDF})$ that the fade slope $\zeta$ is exceeded for a given $A, P(\zeta \mid A)$, can be derived as: 


$$
P(\zeta \mid A)=\frac{1}{2} \frac{\left(\zeta / \sigma_{\zeta}\right)}{\pi\left(1+\left(\zeta / \sigma_{\zeta}\right)^{2}\right)}-\frac{\arctan \left(\zeta / \sigma_{\zeta}\right)}{\pi}
$$

The conditional probability $P(|\zeta| \mid A)$ that the absolute value of the fade slope $\zeta$ is exceeded for a given $A$ is:

$$
P(|\zeta| \mid A)=\int_{-\infty}^{-\zeta} p(x \mid A) d x+\int_{\zeta}^{\infty} p(x \mid A) d x=\frac{1}{2} \frac{\left(|\zeta| / \sigma_{\zeta}\right)}{\pi\left(1+\left(|\zeta| / \sigma_{\zeta}\right)^{2}\right)}-\frac{2 \arctan \left(|\zeta| / \sigma_{\zeta}\right)}{\pi}
$$

The model of (2.41) was tested against data between $12.5 \mathrm{GHz}$ and $50 \mathrm{GHz}$ and the results show a good match to the shape of the fade slope cumulative distribution function as well as its variation with attenuation $A$, interval length $\Delta t$ and $3 \mathrm{~dB}$ cut-off frequency of the low-pass filter $f_{B}$. Equations (2.45) and (2.46) are represented in Figures 2.4 and 2.5 respectively.

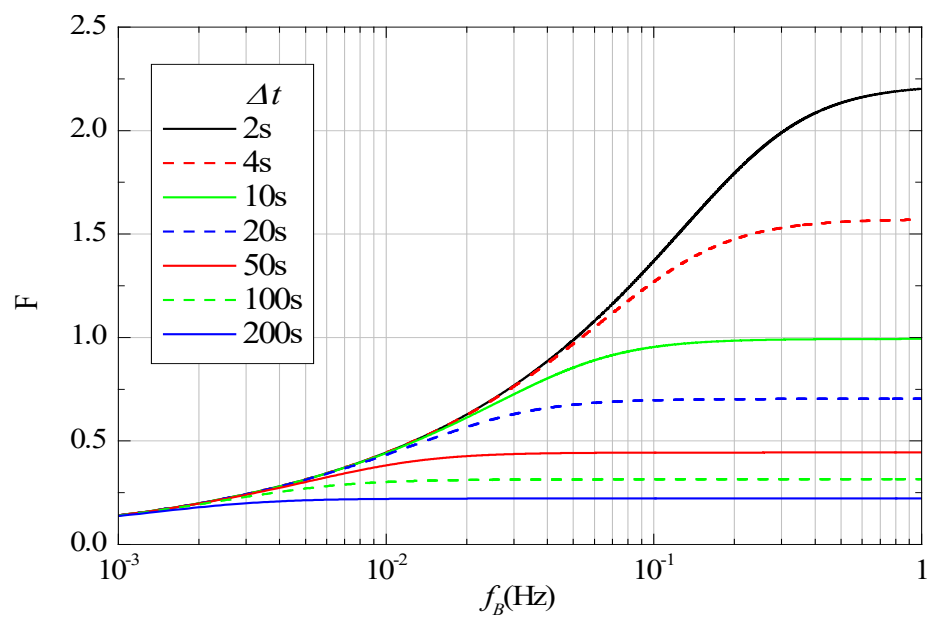

Figure 2.3. Factor $F\left(f_{B}, \Delta t\right)$.

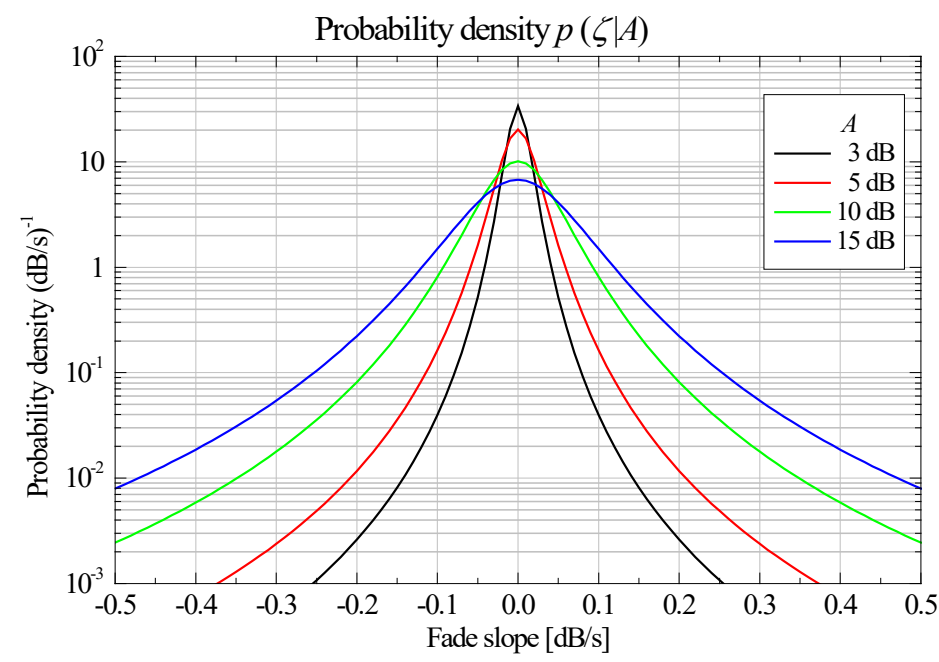

Figure 2.4. Conditional distribution of fade slope for different attenuations with $\Delta t=2 \mathrm{~s}\left(f_{B}=0.025 \mathrm{~Hz}\right)$. 


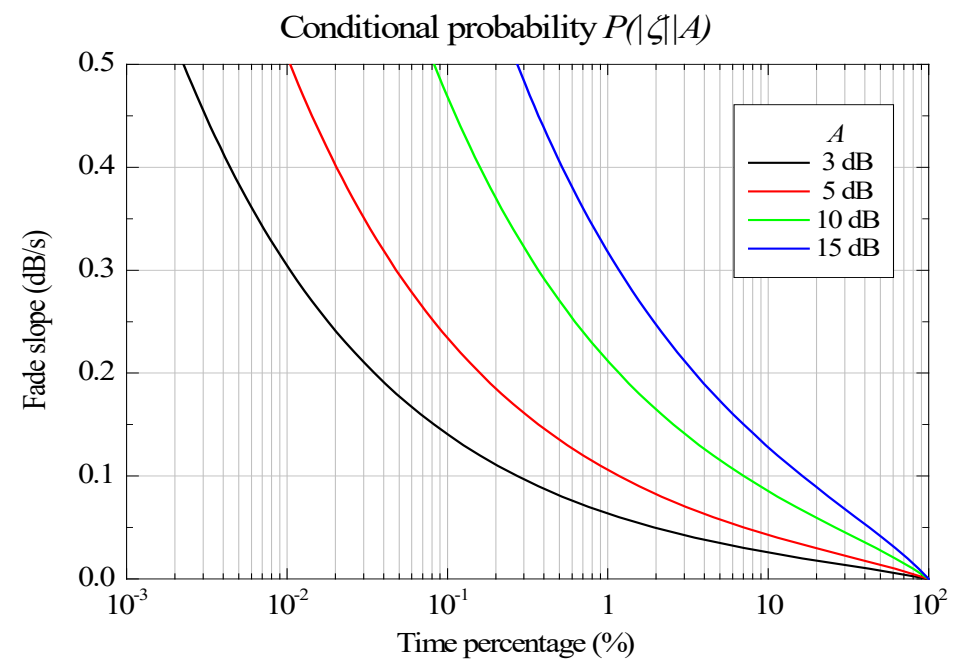

Figure 2.5. Cumulative distribution function of fade slope for different attenuations with $\Delta t=2 \mathrm{~s}\left(f_{B}=0.025 \mathrm{~Hz}\right)$.

\subsection{Propagation impairment mitigation techniques (PIMT): Diversity}

The methods used to counteract the adverse propagation effects and in that way to contribute in maintaining certain service availability are named propagation impairment mitigation techniques (PIMT). These methods take into account the operational frequency bands, the system performance objectives and the main system design parameters (such as network structure, payload type, multiple access techniques, etc.). PIMT can be classified into three major categories [124]:

\section{EIRP (Effective Isotropic Radiated Power) control techniques.}

- The Up-Link/Down-Link Power Control (ULPC/DLPC) consists in varying either the carrier power or the antenna gain for compensating the power losses due to the propagation effects and maintaining a desired power level at the receiver. This technique is used to restore the link by increasing the EIRP during a fade event.

\section{Adaptive transmission techniques.}

- The Adaptive Coding and Modulation (ACM) is used to compensate fades by changing the coding and/or modulation to a more efficient one; or by reducing the binary rate instead.

\section{Protection by diversity techniques.}

Diversity techniques have as an objective to redirect the information through the network in order to avoid problems caused by atmospheric impairments, obtaining in that way a diversity gain. This gain is achieved by transmitting the information through different radio-electric paths; hence fades that affect the paths are different. Several parameters can be used to form a different radio-electric path, such as the frequency, the 
location, the satellite orbit and the time. The diversity techniques commonly used in satellite links are:

- Frequency Diversity. Secondary equipment on board the satellite capable to transmit in another frequency band is necessary to use this technique. When a fade event occurs, the transmitter switches to the lower frequency, less sensitive to atmospheric effects. In order to analyze this diversity technique, it is necessary to have in the ground station at least two beacon receivers very close together, each one working at different frequencies and both oriented to the same satellite. This configuration allows the assessment of the attenuation dependence on the frequency.

- Site diversity $(S D)$. This technique is oriented to change the used slant path, thus it can be applied only to the Fixed Satellite Service (FSS). It takes advantage of the statistical independence between fades that can affect two ground stations separated a distance longer than a convective rain cell zone (at least $10 \mathrm{~km}$ ). The ground station with better weather conditions will be used in the communication, and the information is retransmitted to the desired final destination using a terrestrial link. To evaluate this technique, two receiving ground stations separated a certain distance, oriented to the same satellite and working at the same frequency are required. A configuration example is shown in the left part of Figure 2.6.

- Orbital diversity $(O D)$ (or satellite diversity). This technique allows that the ground station selects one between different satellites and defines a reorientation strategy from a network point of view — similar to SD - , thus it can be applied only to FSS. The use of OD is less expensive than SD, because satellite switching is carried out in the same ground station; although the decorrelation that can be achieved with this technique is lower than for SD.

- Time diversity $(T D)$. It takes advantage of the time variant nature of the signal to retransmit the information in a more convenient time. An example is shown in the right part of Figure 2.6.

Site diversity scheme

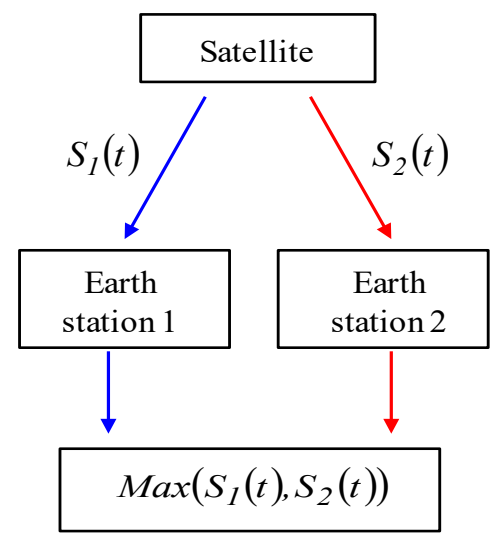

Time diversity scheme

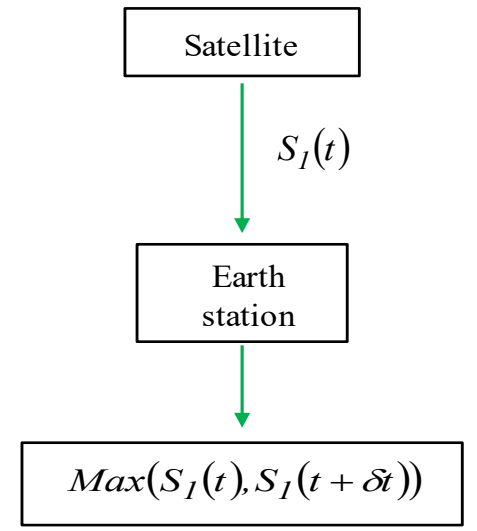

Figure 2.6. Examples of the diversity schemes: site diversity (SD) and time diversity (TD), with $\delta t$ being the time delay. 
These techniques can be activated in the physical layer (layer 1) in the case of frequency, orbital and site diversity, or in the link layer (layer 2) in the case of TD.

The theoretical performance of the ACM and power control can be evaluated using firstorder statistics (mostly of attenuation), whereas prediction methods of second-order statistics (fade slope, fade duration, inter-fade duration and instantaneous frequency scaling) must be used to design PIMT control loops. On the other hand, specific prediction methods must be applied to assess the theoretical performance of the diversity techniques.

The increase of the useful received signal level obtained by using a diversity technique in comparison with the signal level obtained without using that technique is called as diversity gain. The achievement of a diversity gain allows a reduction in the outage probability for a given attenuation [125] — or in a complementary way, a reduction of the attenuation for a given outage probability.

First- and second-order statistical distributions are required in order to define a time series synthetic generator (a channel model synthesizer), as was proposed in [126], to be used for simulating the system and testing the PIMTs. The development of channel models capable to reproduce the spatial and temporal correlation of the atmospheric attenuation in a specific satellite coverage area has then a particular interest.

The performance of PIMT based on diversity can be evaluated with prediction models of frequency scaling for frequency diversity, and with specific models of diversity gain for site, OD and TD.

Due to the available equipment (two satellite beacon receivers working at different frequencies, one site) TD is the only diversity technique that can be studied in a direct manner in this Thesis. OD can be analyzed if some frequency scaling procedures are taken into consideration. Both techniques are addressed in the following sub-sections.

\subsubsection{Time diversity}

This type of diversity is classified as a layer 2 technique, and is based on the message retransmission rather than on mitigating the fade events. Two different techniques can be classified as layer 2: the Automatic Repeat Request (ARQ) and time diversity. With the ARQ the message is re-sent at certain random times until it reaches the receiver satisfactorily. On the other hand, the time diversity technique uses propagation prediction models with the objective of estimating the appropriate time to re-send the message without a request (this is it main difference with ARQ). If deep fade conditions occur, it may be preferable to adjust the re-sending time to an appropriate value to achieve an efficient performance over those conditions. This technique is adequate to specific applications - such as data transfer from geostationary satellites, deep space links, etc. - that can tolerate a long time delay and do not require a real time communication, assuming that the user can wait for the end of a fade event before receiving the required information, thus its use is limited by the maximum delay that the application can tolerate and the buffering capacity onboard the satellite. 
As was pointed out in [127], TD can be taken into consideration to be used in Ka-band and above, due to the wider available bandwidth and the major influence of rain attenuation in these bands. Moreover, its effectiveness lies in taking advantage of the rain attenuation decorrelation (after few minutes in large fades and after few tens on minutes in small fades). Then, by resending the information more than once with a suitable time delay allows that the receiver can select the best received signal in order to reduce the link outage.

The main difference between TD and other diversity techniques is the use of a single link, meaning that it works with the same propagation channel delayed a time interval $\delta t$ (see Figure 2.6).

To assess the TD performance in terms of attenuation, the conditional joint attenuation $\mathrm{CCDF}$ with a time delay $\delta t$ is used $[\mathbf{1 2 8}]$ :

$$
P_{\text {outage }}=P\left((A(t), A(t+\delta t))>A_{t h}\right)
$$

Where:

$$
\begin{aligned}
A(t) & : \text { Attenuation time series without TD. } \\
A(t+\delta t) & : \text { Attenuation time series with a time delay } \delta t . \\
A_{t h} & : \text { Attenuation threshold. }
\end{aligned}
$$

This equation can be rewritten as:

$$
P_{\text {outage }}=P\left[\min (A(t), A(t+\delta t))>A_{t h}\right]
$$

The lowest attenuation value is obtained by comparing the original attenuation time series with a version of itself delayed $\delta t$. Figure 2.7 shows a diagram of this procedure and Figure 2.8 shows an example for a specific day.

Time

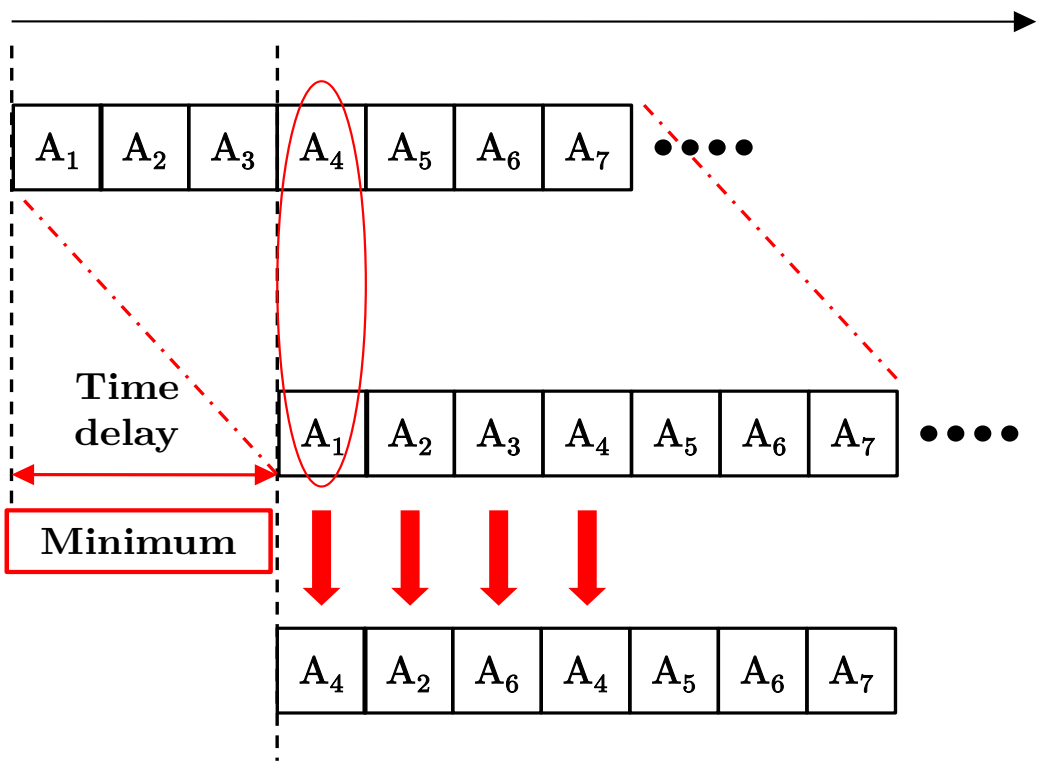

Figure 2.7. Schematic of a time diversity algorithm [128]. 


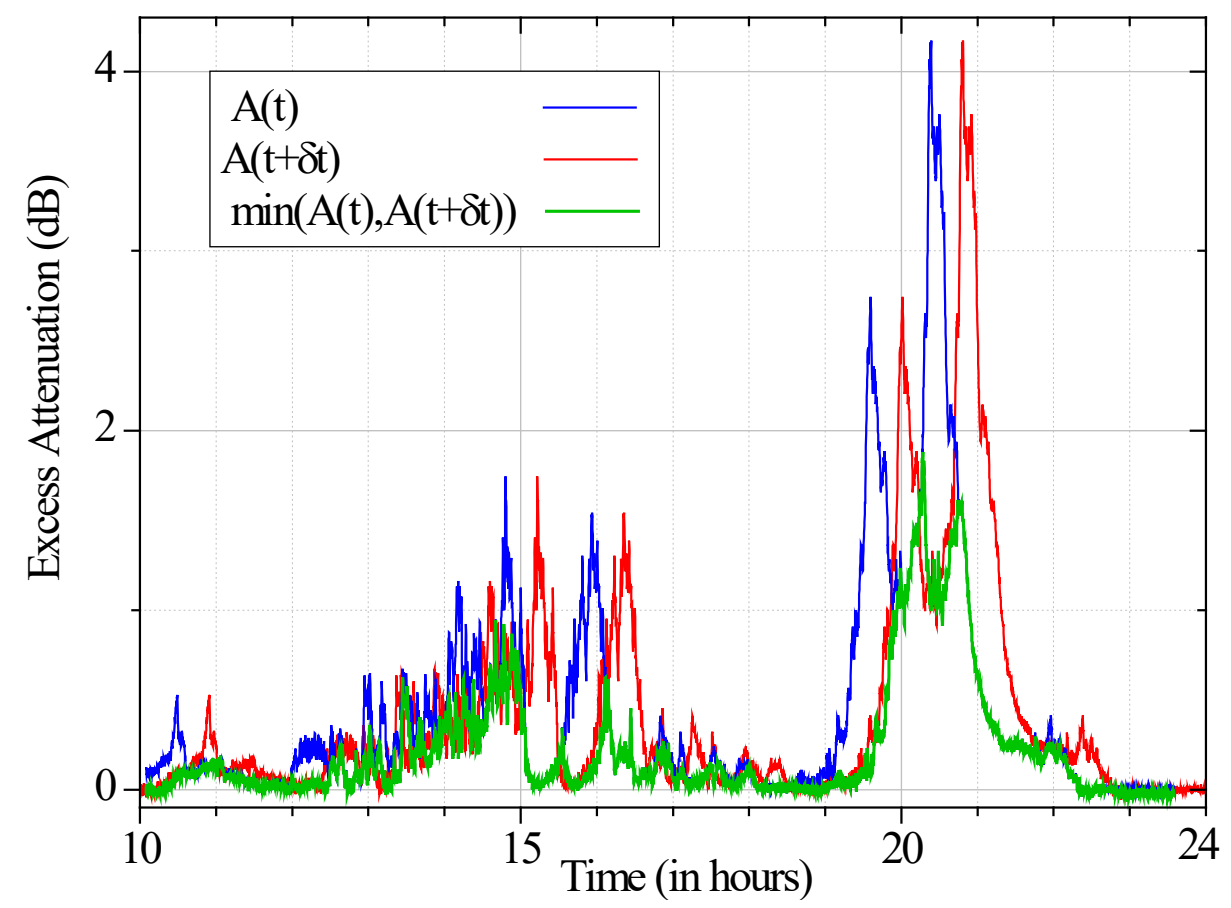

Figure 2.8. Example of TD technique (from 10 to $24 \mathrm{~h}$ of February 23, 2017 of Alphasat beacon signal) with $\delta t=30 \mathrm{~min}$.

The quality and reliability of the TD statistics depend on the measurements availability, since the lack of data in the original time series is duplicated in the final TD series, as Figure 2.9 shows.

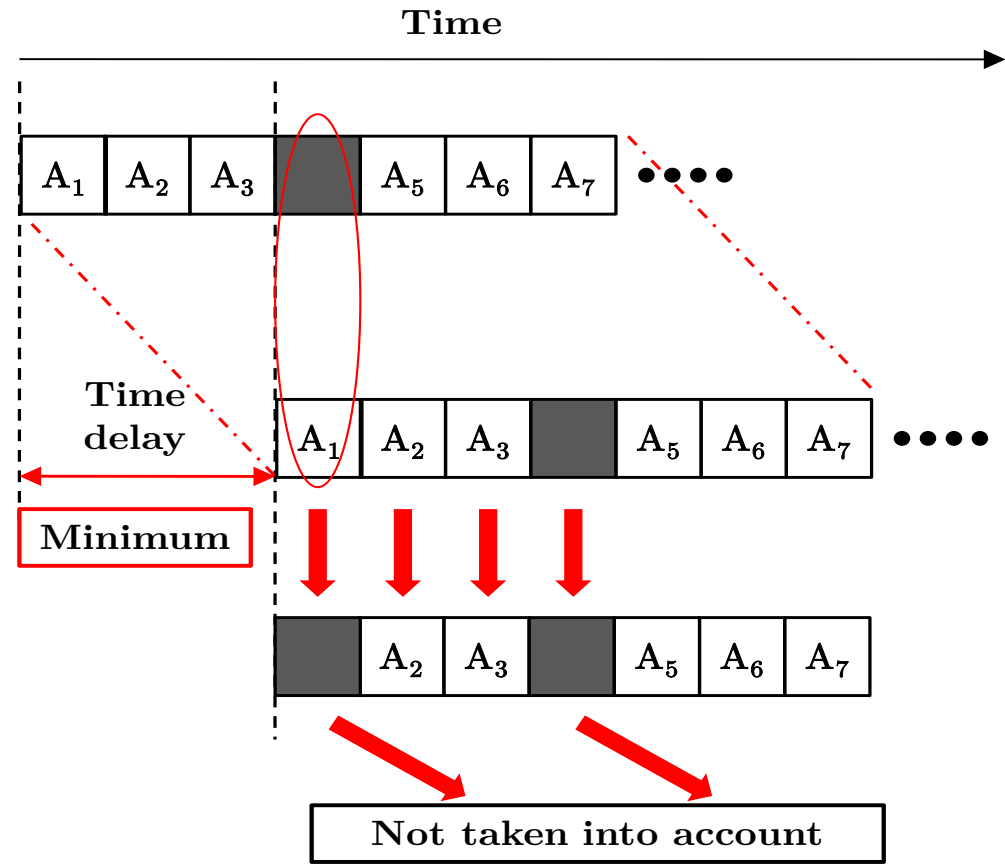

Figure 2.9. Possible missing data duplication in the TD algorithm.

Then, the diversity gain for a given excess probability $p$ and a time delay $\delta t$ is:

$$
G(\delta t, p)=A(0, p)-A(\delta t, p) \quad[d B]
$$


where $A(0, p)$ is the attenuation distribution without a delay at probability $p$ and $A(\delta t, p)$ is the attenuation jointly exceeded with probability $p$ in the original $A(t)$ and $A(t+\delta t)$.

The importance of TD is that it allows a diversity gain by means of an accurate retransmission policy with a low system cost, since it does not require additional equipment and complex synchronization processes due to its application to a single satellite link.

There are not widely validated models for TD, with model reports being scarce in comparison with other mitigation techniques. Among the first available models there is one proposed by Ismail and Watson [129], that was developed for specific conditions: a beacon receiver of $12 \mathrm{GHz}$ in Malaysia, equatorial region where the precipitations are very strong. Thus, the model application is limited and its global use has not been carried out. Four of the more recent and used TD models are studied in this Thesis, which are: the Matricciani, the Joint Probability, the Greece and the ONERA models.

\subsubsection{Matricciani TD Model}

Matricciani proposed in [130] a model to evaluate the use of TD for mitigating the rain attenuation effects in satellite links that do not operate in real time. The method is based on rain attenuation time series simulated in the range from 10 to $100 \mathrm{GHz}$, generated by applying the Synthetic Storm Technique (SST) to rain measurements data of a seven-year period (1994-2000) from Spino d'Adda, Italy. The following equation for the TD gain is proposed:

$$
G(p)=\left(0.65 e^{-0.036 f}+0.38\right)\left(1-e^{-0.09 T\left(1-e^{-0.44 A(p)}\right)}\right) A(p)
$$

In (2.50) two parameters are taken into consideration: the first is frequency $f(\mathrm{GHz})$ dependent and the second is rain attenuation $A(\mathrm{~dB})$ dependent. The time delay $T(\mathrm{~min})$ goes from 0 to 60 minutes. The method predictions were found as optimistic from the comparison with the measurements of Italsat beacon at $18.7 \mathrm{GHz}$.

\subsubsection{Joint Probability Model}

The Joint Probability TD model described in $[\mathbf{1 3 1}],[\mathbf{1 3 2}]$ is based on the medium scale ergodicity of rain, and assumes that rain variability is similar in space and time in the range of tens of kilometers. Then, it is possible to use a model similar to the ones proposed by Paraboni and Barbaliscia [133] or the ITU-R [16] for site diversity, which starts by describing the CCDF of the rain attenuation with a log-normal law as:

$$
P\left(A>A_{t h}\right)=\frac{1}{\sigma \sqrt{2 \pi}} \int_{A_{t h}}^{\infty} \frac{1}{A} \exp \left[-\frac{1}{2}\left(\frac{\ln A-m}{\sigma}\right)^{2}\right] d A
$$

where $m$ and $\sigma$ are the mean and the standard deviation of the distribution, respectively.

Then, this model describes the joint CCDF obtained after the TD processing as the combination of two correlated log-normal distributions, using a time correlation coefficient $\rho$ : 


$$
P_{\text {binorm }}\left(A_{0}>A_{t h}, A_{\delta t}>A_{t h}\right)=\frac{1}{2 \pi \sqrt{1-\rho^{2}}} \int_{X_{0}}^{\infty} \int_{X_{\delta t}}^{\infty} \exp \left[-\left(\frac{x_{0}^{2}-2 \rho x_{0} x_{\delta t}+x_{\delta t}^{2}}{2\left(1-\rho^{2}\right)}\right)\right] d x_{0} d x_{\delta t}
$$

with $x_{0}=\left(\left(\ln A(t)-m_{0}\right) / \sigma_{0}\right)$ and $x_{\delta t}=\left(\left(\ln A(t+\delta t)-m_{\delta t}\right) / \sigma_{\delta t}\right)$ being the normalized attenuation variables for delays of 0 and $\delta t \mathrm{~s}$, respectively; $X_{0}=\left(\left(\ln A_{t h}-m_{0}\right) / \sigma_{0}\right)$, $X_{\delta t}=\left(\left(\ln A_{t h}-m_{\delta t}\right) / \sigma_{\delta t}\right)$, and $m_{0}$ and $\sigma_{0}$ are the mean and the standard deviation of the log-normal distribution of attenuation without $\mathrm{TD}$, and $m_{\delta t}$ and $\sigma_{\delta t}$ are the mean and the standard deviation of the log-normal distribution of the attenuation with a delay $\delta t$. The correlation coefficient between the two attenuation series is $\rho . A_{0}$ and $A_{\delta t}$ are obtained for the same site and are only different by their delay; thus, their statistical means and standard deviations are equal, i.e. $m_{0}=m_{\delta t}$ and $\sigma_{0}=\sigma_{\delta t}$, and the limits in the integral equation $X_{0}$ and $X_{\delta t}$ will also be the same.

The coefficient $\rho$ can be calculated by quadratic regression, minimizing numerically $\varepsilon$, which represents the difference between the bi-normal integral equation of (2.52) and the measured values $P_{\text {measured }}$ :

$$
\varepsilon=\sum_{\% \text { of } t}\left|P_{\text {binorm }}\left(A_{0}>A_{t h}, A_{\delta t}>A_{t h}\right)-P_{\text {measured }}\left(A_{0}>A_{t h}, A_{\delta t}>A_{t h}\right)\right|^{2}
$$

To model the dependency of the TD correlation coefficient with respect to $\delta t$, an exponential function is proposed, as the one proposed for SD correlation with respect to the distance in $[\mathbf{1 3 4}]$ and $[\mathbf{1 3 5}]$ :

$$
\rho=\alpha \cdot e^{-\tau_{1} \delta t}+(1-\alpha) e^{-\left(\tau_{2} \delta t\right)^{2}}
$$

This relatively simple equation depends on three parameters: $\alpha, \tau_{1}$ and $\tau_{2}$. The model does not include procedures for the prediction of TD for new links with different geographical and climatological characteristics from the set of locations used for its development.

\subsubsection{Greece Model}

The first model steps were introduced by Arapoglou et al. in [136], later summarized in the Greece contribution to the ITU-R Working Groups 3K (Point-to-area propagation) and 3M (Point-to-point Earth-space propagation) [137] and published in [138].

The rain attenuation is modeled as a time random variable $A(t)$, where a rain event follows a stochastic model [139] as:

$$
\frac{d A(t)}{d t}=A(t) \beta\left(S_{a}^{2}-\left(\ln A(t)-\ln A_{m}\right)\right)+S_{a} A(t) \sqrt{2 \beta} \cdot n(t)
$$

In (2.55), $\left(A_{m}, S_{a}\right)$ are the statistical mean and standard deviation, respectively, of the long-term log-normal distribution, adopted to approximate the rain attenuation statistics along the path; and $\beta\left(\mathrm{s}^{-1}\right)$ is a statistical parameter that depends on climatological conditions and geometrical and operational link parameters (as frequency and distance) and describes the dynamical properties of the rain attenuation in a propagation path. A white noise stochastic process is introduced with $n(t)$. The parameters $\left(A_{m}, S_{a}\right)$ can be obtained 
analytically from the rain distribution (empirical o simulated) for each site, using the procedure of the Rec. ITU-R P.1057-4 [140].

From equation (2.55), the conditional probability distribution of rain attenuation can be obtained as:

$$
p\left(A(t), t \mid A_{0}, t_{0}\right)=\frac{1}{\sqrt{2 \pi} S_{a}(\Delta t) A(t)} \exp \left[-\frac{\left(\ln (A(t))-\ln \left(A_{m}(\Delta t)\right)\right)^{2}}{2 S_{a}(\Delta t)^{2}}\right]
$$

where:

$$
\begin{gathered}
A_{m}(\Delta t)=A_{m}^{[1-\exp (-\beta \Delta t)]} A\left(t_{0}\right)^{\exp (-\beta \Delta t)} \\
S_{a}(\Delta t)=S_{a} \sqrt{1-\exp (-2 \beta \cdot \Delta t)}
\end{gathered}
$$

and:

$$
\Delta t=\left|t-t_{0}\right|
$$

The joint probability distribution that quantifies the TD system performance is:

$$
P_{T D}=P_{r}\left\{A(t) \geq a, A\left(t_{o}\right) \geq a\right\}=\int_{a}^{\infty} \int_{a}^{\infty} p\left(A\left(t_{o}\right)\right) \cdot p\left\{A(t), t \mid A\left(t_{o}\right), t_{o}\right\} d A(t) d\left(A\left(t_{o}\right)\right)
$$

with:

$$
p\left(A\left(t_{o}\right)\right)=\frac{1}{\sqrt{2 \pi} S_{a} \cdot A\left(t_{0}\right)} \exp \left[-\frac{\left(\ln A\left(t_{0}\right)-\ln A_{m}\right)^{2}}{2 S_{a}^{2}}\right]
$$

\subsubsection{ONERA Model}

The model is analogous to the site diversity model proposed by Boulanger in [141], and is based on the analytical approximation of the joint probability, $P_{\delta t}=P\left(A(t)>A_{t h}, A(t+\right.$ $\left.\delta t)>A_{t h}\right)$, that the attenuation exceeds simultaneously a given threshold $A_{t h}$ in times $t$ and $t+\delta t$.

The rain attenuation time series properties described in $[\mathbf{1 4 2}]$ are used to approximate analytically the joint probability of attenuation exceedance. The approximation is based on the conversion of a stationary correlated Gaussian process $G(t)$ into a rain attenuation process $A(t)$ as Rec. ITU-R P.1853-1 recommends [126]. Since this model uses the conditioned rain distribution, the mean and the standard deviation obtained are different than those obtained for the previous models.

The input parameters are:

$$
\begin{aligned}
&\left.A_{t h}: \text { Attenuation threshold (in } \mathrm{dB}\right) . \\
& P_{0}: \text { Rain attenuation probability (\%), which can be estimated using Rec. } \\
& \text { ITU-R P.837-7 [57]. } \\
& m: \text { Mean of the conditioned rain attenuation log-normal distribution. }
\end{aligned}
$$


$\sigma$ : Standard deviation of the conditioned rain attenuation log-normal distribution.

$\beta$ : Parameter that describes the rain attenuation dynamics (in $\mathrm{s}^{-1}$ ), for a given location (like in the previous model) and is calculated with this model parameters, a general value is provided in Rec. ITU-R P.1853-1.

The main characteristic of this model is that it uses the probability of occurrence $P$ conditioned to the rain attenuation probability $P_{0}$ :

$$
P=\frac{P_{0}}{100} Q\left(\frac{\ln \left(A_{t h}\right)-m}{\sigma}\right)
$$

Since this model cannot be easily consulted in the open literature, the step-by-step procedure is given in Annex 2.

\subsubsection{Orbital diversity}

For using OD, at least two satellite receivers working in the same frequency band and oriented to different orbital positions (see Figure 2.10) are needed. The separation angle between links $\theta$ is the main configuration parameter, which rules the correlation between the signals received from each satellite during an attenuation event. The improvement of one link with respect to the others (there can be more than two links in the scheme) is obtained by the de-correlation of rain attenuation between the paths. It must be taken into account that links may have different elevation angles $\left(\phi_{1}\right.$ and $\phi_{2}$ in Figure 2.10), and then for a higher elevation angle corresponds a smaller path $L_{i}$ and a lower attenuation - under equivalent atmospheric conditions. $H_{1,2}$ represent the average annual rain height with respect to the sea level for each path; they can usually be taken as equal.

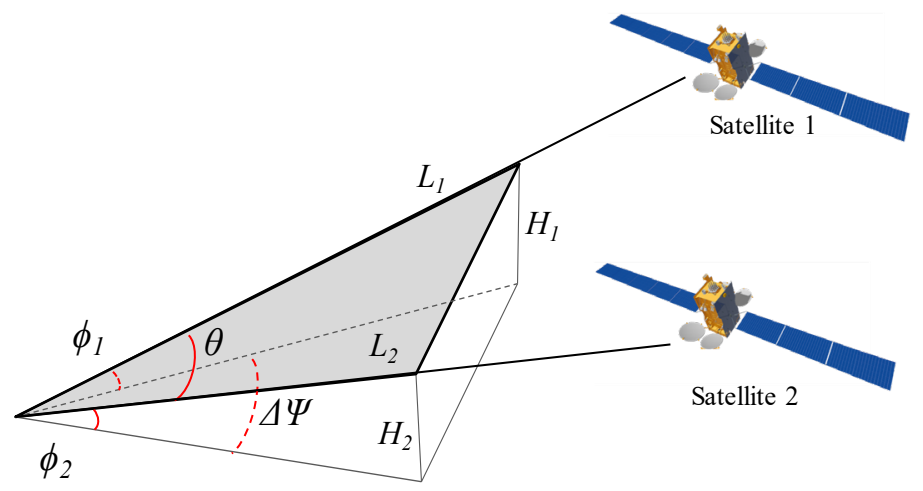

Figure 2.10. Two-satellite orbital diversity schematic.

The angle $\theta$ can be approximated with the equation given in $[\mathbf{1 4 3}]$ :

$$
\cos \theta=\sin \phi_{1} \sin \phi_{2}+\cos \phi_{1} \cos \phi_{2} \cos \Delta \Psi
$$

$\Delta \Psi$ being the difference between the azimuth angles. 
The orbital diversity gain $G_{O D}$ (in $\mathrm{dB}$ ) is defined as the difference in a certain moment between the exceeded attenuation for a $i$-sm link, $A_{S_{i}}$, and the jointly exceeded attenuation for all the links in the diversity scheme, $A_{D}$ :

$$
G_{O D_{i}}(\mathrm{~dB})=A_{S_{i}}(\mathrm{~dB})-A_{D}(\mathrm{~dB}) \quad(i=1,2, \ldots)
$$

The minimum value of attenuation in all links for a given instant is:

$$
A_{D}(\mathrm{~dB})=\min \left\{A_{S_{1}}, \ldots, A_{S_{i}}\right\}
$$

In [144] it is pointed out that, compared with other techniques, the diversity gain that can be obtained with OD may be more expensive in terms of involved satellite number, ground terminal equipment, and system architecture and operation.

In the open literature, there are few references to OD experiments. The study carried out in Fucino, Italy [145] with the SIRIO and OTS satellites for 15 rain events at $12 \mathrm{GHz}$ is one of the most referenced [143] [146]. Another experiment was done in Spino d'Adda, Italy [146] with the Italsat and the Olympus satellites at $20 \mathrm{GHz}$.

Another OD study was carried out in Aveiro, Portugal [147] with links operating at different frequencies and this situation was overcome with a frequency scaling procedure from the lower frequency $(20 \mathrm{GHz})$ to the higher one $(40 \mathrm{GHz})$. In this procedure, a power-law scaling was applied, the appropriate scaling coefficients were obtained from measurements taken in Vigo, Spain (the two sites share the same climatological conditions) by a regression analysis. In Vigo two beacon receivers at $20 \mathrm{GHz}$ and $40 \mathrm{GHz}$ are available and are oriented to Alphasat, as the $40 \mathrm{GHz}$ receiver available in Aveiro. Then, the coefficients are considered suitable to be used in Aveiro in order to scale the lower frequency measurements. Moreover, it is mentioned in this work that the scaling method of sub-section 2.2.1.3.2 of Rec. ITU-R P.618-13 overestimates the resulting attenuation distribution for medium and high probabilities. Using this inspiration, a procedure has been carried out to evaluate OD in Madrid with the available experiments, as will be presented in Section 5.5.

From the open literature, there are few proposed models for OD estimation, but they have not been widely used or validated and were developed using only frequencies limited to the $\mathrm{Ku}$ - and Ka-bands, hence their use in the Q-band may not be appropriate. Two of these models are presented here.

\subsubsection{Matricciani OD Model}

An OD model was developed by Matricciani [148] using data from Fucino, Italy. This model uses some values that were derived from radar measurements. It only needs the statistical characteristics of a single link — besides a correlation coefficient - to predict the joint probability of exceedance. The distribution function of the OD system is given as a function of the single link probability $P_{S}$ :

$$
P_{D}=P_{S}^{2-\rho}
$$


$P_{S}$ is estimated by using the mean and the standard deviation of the single link distribution for the normalized values of attenuation, as is described in [148]. The correlation coefficient $\rho$ between the logarithms of the attenuation on the links is:

$$
\rho=\frac{1}{\sigma^{2}} \ln \left[r(L, \theta)\left(\exp \left(\sigma^{2}\right)-1\right)+1\right]
$$

with $\sigma^{2}$ being the standard deviation obtained by the model procedure, and $r$ is a correlation coefficient between the single-link attenuation distributions (in $\mathrm{dB}$ ):

$$
r(L, \theta)=I(L, \theta) / I(L, 0)
$$

where:

$$
I(L, \theta)=\int_{0}^{L_{2}} \int_{0}^{L_{1}} \exp \left(-\beta\left(x^{2}+y^{2}-2 x y \cos \theta\right)^{1 / 2}\right) d x d y \quad\left[\mathrm{~km}^{2}\right]
$$

and:

$$
\begin{array}{ll}
L_{1} & : \text { The rainy path length for link } 1 . \\
L_{2} & : \text { The rainy path length for link } 2 . \\
\beta^{-1} & \text { : De-correlation distance. }
\end{array}
$$

\subsubsection{NTUA Model}

Kanellopoulos and Repas proposed an OD model in [149], using a rather complex procedure to obtain the joint probability of exceedance of attenuation. The presented results have a moderate precision. A complementary study was reported in [150] for an unbalanced system, simplifying the formulation; this is the one used in this Thesis. Both reports use a log-normal distribution for the prediction of the exceedance probabilities for each of the links and the joint exceedance probability. On the other hand, a model that uses a gamma distribution instead of the log-normal one is proposed in [151], reporting an improvement in the description of the distributions.

This method proposes the following equation to estimate the joint exceedance probability function between the attenuation of two links:

$$
P_{12}(A)=\frac{1}{2} \int_{u_{0}}^{\infty} \frac{1}{\sqrt{2 \pi}} e^{-u^{2} / 2} \cdot \operatorname{erfc}\left[\frac{v_{0}-\rho_{n} u}{\sqrt{2\left(1-\rho_{n}^{2}\right)}}\right] d u
$$

where the exceeded levels $u_{0}$ and $v_{0}$ are calculated from the probabilities of exceedance $P_{1,2}(A)$ of attenuation for links 1 and 2 :

$$
\begin{aligned}
& u_{0}=\sqrt{2} \operatorname{erfc}^{-1}\left[P_{1}(A)\right] \\
& v_{0}=\sqrt{2} \operatorname{erfc}^{-1}\left[P_{2}(A)\right]
\end{aligned}
$$

with $\rho_{n}$ being a correlation coefficient between the individual probability distributions given by: 


$$
\rho_{n}=\frac{1}{\sigma_{1} \sigma_{2}} \ln \left[\rho \sqrt{\left(\exp \left(\sigma_{1}^{2}\right)-1\right)\left(\exp \left(\sigma_{2}^{2}\right)-1\right)}+1\right]
$$

$\sigma_{1}$ and $\sigma_{2}$ being the standard deviations of the attenuation distributions of the respective single links and $\rho$ the correlation coefficient between the link attenuation distributions, which can be calculated with expressions provided in [150]. An important parameter in all the process is the characteristic distance $G$ (used in the previously cited expressions) that depends on the rainfall medium. The reference value provided for Fucino, Italy -i.e. $G=1.5$ $\mathrm{km}$ - is used here due to the similarity in latitude and altitude between Fucino and Madrid.

A model with a reduced complexity based on the previous one was reported by Panagopoulos and Kanellopoulos [152], giving very good approximation in [147] for probabilities higher than $0.03 \%$ for regions with high rain rates.

\subsection{Variability of propagation statistics}

Most of the models analyzed until now are based on experimental results, from which the model algorithms or equations were derived by regression or correlation techniques. Special care must be taken with the experimental data used in the modeling process due to the high statistical variability of the results of different years or from different locations. Long-term experimental data are very important in the development of models, since they allow assessing the variability of a certain parameter or phenomenon over time and space and, from that, estimating the average behavior in a given period or climatological zone.

There are only a few models developed to predict the variability of the rainfall rate and rain attenuation statistics produced in satellite propagation $[\mathbf{1 5 3}],[\mathbf{1 5 4}]$, and they are not adequate to describe the fade dynamics variability. The statistical variability of rainfall rate $[\mathbf{1 5 5}],[\mathbf{1 5 6}]$ or rain attenuation $[\mathbf{1 5 7}],[\mathbf{1 5 8}],[\mathbf{1 5 9}]$ have been studied in several articles using experimental data, being more scarce those that analyze fade $[\mathbf{1 6 0}]$ or inter-fade duration variability.

The annual CCDFs of rainfall rate $(R)$ or rain attenuation $(A)$ are used to evaluate their year-to-year variation. A very important issue is the number of measured years required to evaluate with a high degree of accuracy the natural variation of rain and other climatological phenomena, and their effects on propagation. In [155] it is concluded that seven is the minimum number of years for which the average individual distributions are included in the 5-95\% confidence interval in a long-term distribution of 49 years, although ten years would be desirable, in spite of the difficulty to find propagation experiments with such long duration. Having the CCDFs of the variable of interest ( $R$ or $A$ ) for a number of years, the variability can be characterized in two different and complementary ways: by analyzing the probability $p_{i}(R)$ or $p_{i}(A)$ of exceeding a given threshold $R$ or $A$ for the $i$-years; or by analyzing the values of $R_{i}(p)$ or $A_{i}(p)$ associated to certain probabilities $p$ in the different years. The latter form is recommended by Rec. ITU-R P.678-3 [154], which provides a method to estimate the annual variance $\sigma^{2}$ of the probability $p$ for percentages of outage time between 0.01 and $2 \%(0.0001 \leq p \leq 0.02)$ and for frequencies between 12 and $50 \mathrm{GHz}$, as follows: 


$$
\sigma^{2}(p)=\sigma_{C}^{2}(p)+\sigma_{E}^{2}(p)
$$

where:

$$
\begin{aligned}
& \sigma_{E}^{2}(p) \quad: \text { The variance of estimation. } \\
& \sigma_{C}^{2}(p) \quad \text { : Inter-annual climatological variance. }
\end{aligned}
$$

To obtain $\sigma^{2}$, it is necessary to know:

$p \quad$ : Outage probability $(0 \leq p \leq 1)$.

$r_{c} \quad$ : Climatic ratio, available as digital maps in a supplementary file of Rec. ITU-R P.678-3, obtained from long-term databases.

Then, for a given outage probability $p$, the following parameter is calculated:

$$
C=\sum_{i=-N+1}^{N-1} c_{U}(i \Delta t, p)
$$

where:

$$
\begin{aligned}
& N=525960 \text { (minutes of a year of } 365.25 \text { days) } \\
& \Delta t=60 \\
& c_{U}(i \Delta t, p)=\exp \left(-a \cdot|i \Delta t|^{b}\right)
\end{aligned}
$$

and:

$$
\begin{aligned}
& b=b_{1} \cdot \ln (p)+b_{2} \\
& a=0.0265\left(s^{-1}\right) \\
& b_{1}=-0.0396 \\
& b_{2}=0.286
\end{aligned}
$$

The variance of estimation $\sigma_{E}^{2}(p)$ is calculated as:

$$
\sigma_{E}^{2}(p)=\frac{p(p-1)}{N} C
$$

Later, the variable $r_{c}$ for the four nearest points in latitude (Lat) and longitude (Lon) to the desired geographical coordinates is extracted, and from these four $r_{c}$ values, the $r_{c}($ Lat, Lon $)$ is obtained by means of a bilinear interpolation as described in [60].

The inter-annual climatological variance $\sigma_{C}^{2}(p)$ is:

$$
\sigma_{C}^{2}(p)=\left(r_{c}(\text { Lat }, \text { Lon }) \cdot p\right)^{2}
$$

This ITU-R Recommendation also gives indications to work with estimated CCDF values (obtained from a rainfall rate or a rain attenuation prediction model) instead of the experimental ones. In such cases, an additional error $\sigma_{M}^{2}(p)$ due to the predicted CCDF must be taken into account, transforming equation (2.72) into:

$$
\sigma^{2}(p)=\sigma_{C}^{2}(p)+\sigma_{E}^{2}(p)+\sigma_{M}^{2}(p)
$$


The method points out the convenience of referring the analysis to the $68 \%$ confidence interval $[p-\sigma(p), p+\sigma(p)]$, and in that manner to assess the influence of the variance $\sigma^{2}(p)$, since this corresponds to plus or minus one standard deviation around the probability for a normally distributed quantity.

Another way to assess the variability is by using the variability parameter (here named $V(p)$ ), proposed in [156], and employed to compare the individual year distributions $R_{i}(p)$ or $A_{i}(p)$ with the long-term distributions $R_{A Y}(p)$ or $A_{A Y}(p)$. For the rainfall rate distributions:

$$
\begin{array}{ll}
V(p) & =\left[<e_{i}(p)^{2}>\right]^{1 / 2} \\
e_{i}(p) & =\frac{R_{i}(p)-R_{A Y}(p)}{R_{A Y}(p)} \cdot 100 \%
\end{array}
$$

$n$ being the number of years.

For the rain attenuation distributions an analogous equation can be used:

$$
e_{i}(p)=\frac{A_{i}(p)-A_{A Y}(p)}{A_{A Y}(p)} \cdot 100 \% \quad i=1, \ldots, n
$$

These equations have been modified in $[\mathbf{1 6 1}]$ for applying them to the fade duration statistics, i.e. $N(D), T(D), P(D)$ and $F(D)$, having for the fade duration number of events distribution $N(D)$ :

$$
\begin{aligned}
& V\left(D \mid A_{t h}\right)=\left[<e_{i}\left(D \mid A_{t h}\right)^{2}>\right]^{1 / 2} \\
& e_{i}\left(D \mid A_{t h}\right)=\frac{N_{i}\left(D \mid A_{t h}\right)-N_{A Y}\left(D \mid A_{t h}\right)}{N_{A Y}\left(D \mid A_{t h}\right)} \cdot 100 \% \quad i=1, \ldots, n
\end{aligned}
$$

With $N_{A Y}(D)$ being the distribution of the average year. Then, by substituting the statistic of interest in (2.82), the variability of each of the four fade duration statistics can be assessed for a given attenuation threshold.

\subsection{Conclusions}

In this chapter the main first- and second-order statistics regarding attenuation in satellite links have been addressed. Rain is the main attenuation phenomenon in the analyzed bands (the Ka and $\mathrm{Q}$ bands) and has been treated in detail. Also, the most recent rainfall rate prediction methods have been presented. Gas and cloud attenuation, as well as scintillation have been lightly discussed since they are less relevant in these bands. Also, the main prediction methods for each phenomenon have been presented.

Fade and inter-fade duration results are scarce, with limitations in the analyzed number of years and frequencies, particularly in the Q-band. Although there are some fade duration models, their accuracy is limited. Also, there are two modeling techniques (the ONERA and Bråten fittings) that allow describing the fade duration probability of occurrence with certain distribution combinations. They may be used to improve the existing models or to develop new ones. For example, the ITU-R Rec. P.1623-1 fade duration probability of occurrence 
model uses a power-law function for modeling the short durations and a log-normal function for modeling the long durations and the CRC model uses the sum of two log-normal functions. Then it may be useful to compare the coefficients that best fit the available data with those provided by the models or to determine the distribution combination that gives the best estimate.

In the case of inter-fade duration, a model with a combination of three log-normal distributions has been proposed to the ITU-R, but its use has not been widely validated yet. Maybe, the use of such complex combination has its drawbacks or on the other hand, a simpler one may serve properly.

Time and orbital diversity techniques have been discussed, since they can be analyzed with the available equipment. For both cases, several models have been found in the literature; nevertheless, few references were found on this matter. A comparison between these models may serve to address their performances. For deriving orbital diversity results, it seems that some modification must be applied first to the data (since the satellite experiments use different frequency bands), as it has been done in works reported in some references.

Finally, the variability of propagation statistics was addressed. The importance of knowing the variation of the statistics is unquestionable. At the end, models are provided for an average year, but what happens if the analyzed year deviates from this? Having a statistic that describes that variation is of a paramount importance. Although there is a model to predict the rainfall rate and rain attenuation variability, no current model predicts the fade dynamics variability. 


\section{Millimeter-wave indoor channel modeling}

\section{Introduction}

Nowadays, millimeter-wave technology is receiving high attention due to the fact that it is expected that millimeter-wave bands will be used in the next wireless and mobile generation, encompassed by the IMT 2020 and beyond $[\mathbf{1 6 2}]$ — usually named as the fifth generation of mobile communications, 5G. Actually, millimeter-wave frequencies are one of the four main demands identified in order to satisfy the $5 \mathrm{G}$ wireless communication system requirements - i.e. spatial consistency and mobility, diffuse versus specular scattering, very large antenna arrays and millimeter wave frequencies $[\mathbf{7}]$ - and hence must be taken into consideration for channel modeling. The idea is to use bands below and above $6 \mathrm{GHz}$ in a complementary manner in the future systems: low frequencies can be used to provide a wider coverage area or to facilitate indoor penetration, while higher frequency bands could be used in outdoor and indoor deployments with higher capacity but reduced coverage.

Accurate models aid the system designer when assessing the expected coverage inside buildings or areas, ensuring system performance when predictions are close to real values [163]. The estimation of the propagation path loss as a function of distance, frequency and propagation scenario is very important for modeling the radio wave propagation in a given channel. Regarding their construction approach, wireless models can be classified in deterministic, stochastic or empirical. Models with an adequate number of parameters, not excessively complex or computationally expensive, are preferred. 
The standardization organizations usually require and develop models with omnidirectional antennas, in which the propagation parameters - path loss, multipath delays, wave direction of departure (DoD), wave directions of arrival (DoA), etc.- are processed statistically in all the paths. Moreover, omnidirectional channel models are applicable to any system, such as arbitrary MIMO systems or beamformers [164].

There are several propagation model classifications for fixed or mobile horizontal links. An important one is the line-of-sight (LOS) condition: configuration obtained when there is a direct line without obstructions between the transmitter and the receiver. Models must have formulas that describe propagation when conditions are LOS and non-LOS (NLOS). Another classification that can be seen as intermediate between the previous ones is the obstructedline-of-sight (OLOS), in which there are some objects in the transmitter-receiver line or within the First Fresnel Zone.

Other model classification depends on the terminal locations, with two main options: outdoor and indoor. Only scenarios with both terminals inside a building will be considered as indoor (InH, Indoor Hotspot) in this work (this consideration excludes outdoor-to-indoor or indoor-to-outdoor configurations). Besides, only those models that have been tested or developed to work at millimeter-wave frequencies will be treated. A detailed classification including other possible scenarios can be found in [165].

Some experimental campaigns have been $[\mathbf{1 6 6}][\mathbf{1 6 7}]$ - or are being $[\mathbf{1 6 8}][\mathbf{1 6 9}][\mathbf{1 7 0}]$ carried out around the world at millimeter-wave frequencies, either in indoor or outdoor scenarios. Several relevant reports from past campaigns can be found such as: the reports of mmMAGIC [171] (Millimetre-Wave Based Mobile Radio Access Network for Fifth Generation Integrated Communications) from the 5GPPP (5G infrastructure Public Private Partnership), in the framework of the European Union (EU) Research and Innovation Programme HORIZON 2020; the documents from METIS (Mobile and wireless communications Enablers for Twenty-twenty(2020) Information Society) [172] and the white paper of the 4th International Workshop on $5 \mathrm{G} / 5 \mathrm{G}+$ Communications in Higher Frequency Bands (5GCHFB) [173] — with contributions from 15 companies and universities ${ }^{3}$ and hereinafter referred as 5GCM, as was done in [174]. Furthermore, the NYU WIRELESS research team of the New York University (NYU) has produced a great number of research articles and a free online software (http://wireless.engineering.nyu.edu/) —unfortunately for this Thesis, the software is not intended to be used in indoor scenarios (until now, it only includes outdoor scenarios). In these documents the most relevant experiments in indoor millimeter-wave propagation that have been carried out in the last years are summarized. A common goal to all of them was the development of channel models. Also, several journals have included special issues dedicated almost completely to $5 \mathrm{G}$ communications (including antenna designs, communication issues, and channel modeling), as the IEEE Transactions on Antenna and Propagation journal did recently [175].

${ }^{3}$ The contributors are: Aalto University, AT\&T, BUPT, CMCC, Ericsson, Huawei, INTEL, KT Corporation, Nokia, NTT DOCOMO, New York University, Qualcomm, Samsung, University of Bristol and University of Southern California. 
Previous to the 5G-oriented developments, there were several wireless propagation channel models; most of them were developed for frequencies lower than $6 \mathrm{GHz}$, and their use at higher frequencies is questionable once the propagation phenomena change. Still, their assessment at such higher frequencies may be used as a starting point. The following were compared in the study carried out in the framework of the METIS project [176]: the WINNER (Wireless world INitiative New Radio) [177], the WINNER+ (an extension of the previous one) and the 3GPP-3D of the 3 GPP (3rd Generation Partnership Project), the IMT-Advanced of the ITU-R [178], the COST 2100, the Quadriga (QUAsi-Deterministic RadIo channel GenerAtor model) and the IEEE 802.11ad (the only one developed to be used at a millimeter-wave band, specifically in the 60-GHz). The MiWEBA (Millimetre-Wave Evolution for Backhaul and Access) model [179] was also developed to describe the $60-\mathrm{GHz}$ band, but its use in other bands has not been addressed yet.

Besides the testing and upgrading of previous models, other possible approach is the development of new ones that can operate in the entire millimeter-wave range - they should be consistent also for frequencies below $6 \mathrm{GHz}[\mathbf{1 8 0}]$. Due to the fact that the objective of this Thesis is to analyze millimeter-wave propagation in indoor scenarios, only the indoor part of the recently developed millimeter-wave propagation models will be treated in depth. Some studies carried out in the last years concerning indoor scenarios are summarized in Table 3.1, detailing the measured frequencies.

Table 3.1. Experimental campaigns carried out in indoor scenarios (InH) that have been used in the millimeter-wave channel modeling

\begin{tabular}{|c|c|c|}
\hline Organization & Frequencies (in $\mathrm{GHz}$ ) & Multipath Measurements \\
\hline BUPT $[\mathbf{1 8 0}]$ & $14,19.85,20,23,36$ & $\mathrm{x}$ \\
\hline NTT DOCOMO [180] & 19.85 & $\mathrm{x}$ \\
\hline Aalto University $[\mathbf{1 8 0}],[\mathbf{1 8 1}]$ & 28,63 & $\mathrm{x}$ \\
\hline ETRI Korea $[\mathbf{1 8 0}]$ & 28,38 & $\mathrm{x}$ \\
\hline Samsung \& KT Corporation [180] & 28 & $\mathrm{x}$ \\
\hline NYU $[\mathbf{1 8 2}]$ & 28,73 & $\mathrm{x}$ \\
\hline Qualcomm [183] & 29,61 & \\
\hline Durham University [184] & 30,60 & $\mathrm{x}$ \\
\hline Ericsson $[\mathbf{1 7 6}]$ & 58.6 & \\
\hline Bristol University [181] & 60 & $\mathrm{x}$ \\
\hline L'Aquila University [185] & 60 & \\
\hline Huawei $[\mathbf{1 8 0}]$ & 73 & \\
\hline CEA-Leti $[\mathbf{1 8 1}]$ & 82 & $\mathrm{x}$ \\
\hline
\end{tabular}

BUPT: Beijing University of Posts and Telecommunications; NTT: Nippon Telegraph and Telephone; ETRI: Electronics and Telecommunications Research Institute; NYU: New York University, CEA-Leti: Commissariat à l'Energie Atomique et aux Energies Alternatives (CEA)Laboratoire d'électronique des technologies de l'information, KT Corporation: South Korea Telecommunication company.

Moreover, several studies (including some of the above mentioned) are collected in the Report ITU-R P.2406-0 [186] either for outdoor or indoor scenarios (studies related to outdoor-to-indoor environments are available in Report ITU-R P.2346-2 [187]). 
In this chapter, the path loss propagation models developed for millimeter waves in indoor scenario are addressed. Also, the fundamentals of MIMO technique necessary for this work are given.

\subsection{Line of sight probability}

A very useful way to describe the path loss is defining whether LOS or NLOS occurs. At millimeter waves, the diffraction losses, the path loss exponent (PLE, which describes the dependence of the received power with distance) and the shadowing ${ }^{4}$ variance are higher in NLOS conditions than in LOS conditions. Then, LOS conditions are preferable to obtain a higher system performance. For that reason, equations to assess the LOS probability have been developed. These predict the probability or likelihood that the user equipment will have a clear LOS condition with the base station. The LOS probability is modeled as a function of the bi-dimensional separation distance between the transmitter and receiver and is frequencyindependent, once it is based only on the geometry and layout of the scenario. The LOS probability for $\mathrm{InH}$ scenarios of the main $5 \mathrm{G}$ propagation models are detailed next:

3GPP TR 38.901 model [188] [189]

InH-Mixed office:

$$
p_{L O S}=\left\{\begin{array}{cr}
1 & d_{2 D} \leq 1.2 \mathrm{~m} \\
\exp \left(-\left(d_{2 D}-1.2\right) / 4.7\right) & 1.2 \mathrm{~m}<d_{2 D}<6.5 \mathrm{~m} \\
\exp \left(-\left(d_{2 D}-6.5\right) / 32.6\right) \cdot 0.32 & 6.5 \mathrm{~m}<d_{2 D}
\end{array}\right.
$$

InH-Open office:

$$
p_{L O S}^{\text {Open-office }}=\left\{\begin{array}{cr}
1 & d_{2 D} \leq 5 \mathrm{~m} \\
\exp \left(-\left(d_{2 D}-5\right) / 70.8\right) & 5 \mathrm{~m}<d_{2 D}<49 \mathrm{~m} \\
\exp \left(-\left(d_{2 D}-49\right) / 211.7\right) \cdot 0.54 & 49 \mathrm{~m}<d_{2 D}
\end{array}\right.
$$

$5 G C M[180]$

$$
p_{L O S}=\left\{\begin{array}{cc}
1 & d_{2 D} \leq 1.2 \mathrm{~m} \\
\exp \left(-\left(d_{2 D}-1.2\right) / 4.7\right) & 1.2 \mathrm{~m}<d_{2 D}<6.5 \mathrm{~m} \\
\exp \left(-\left(d_{2 D}-6.5\right) / 32.6\right) \cdot 0.32 & 6.5 \mathrm{~m}<d_{2 D}
\end{array}\right.
$$

mmMAGIC [190]

$$
p_{L O S}=\left\{\begin{array}{cc}
1 & d_{2 D} \leq 1.2 \mathrm{~m} \\
\exp \left(-\left(d_{2 D}-1.2\right) / 4.7\right) & 1.2 \mathrm{~m}<d_{2 D}<6.5 \mathrm{~m} \\
\exp \left(-\left(d_{2 D}-6.5\right) / 32.6\right) \cdot 0.32 & 6.5 \mathrm{~m}<d_{2 D}
\end{array}\right.
$$

with $\boldsymbol{d}_{2 \boldsymbol{D}}$ being the 2-D separation distance between the transmitter and the receiver.

${ }^{4}$ As shadowing (or shadow fading) is named the phenomenon which produces a fade in the signal and describes the density of obstacles between transmit and receive antennas and their absorption behavior (both modeled as random numbers), then it depends on the physical environment [191]. 


\subsection{General large-scale path loss models}

The variation over time and frequency is a key element to characterize the wireless channels. There are two types of variations $[\mathbf{1 9 1}][\mathbf{1 9 2}][\mathbf{1 9 3}]$ :

- Small-scale fading, due to the constructive and destructive interferences caused by the signals coming from multiple paths between the transmitter and receiver. These fluctuations occur at the spatial scale of around one carrier wavelength and are frequency dependent.

- Large-scale fading, due to the path loss (as a function of the distance and scenario geometry and usually described in a deterministic manner) and the shadowing produced by large objects. The mean power averaged about ten wavelengths shows these fluctuations when the mobile travels longer distances (typically more than few hundred wavelengths) and is typically frequency independent within a wide range.

Both can be well described statistically by a mean value of the received power in each case (which is a local value in the case of small-scale fading) and the statistics of the fluctuations around this mean.

Large-scale propagation models predict and characterize the average signal strength for a given transmitter-receiver configuration. They do not deal with the fast signal fluctuations in amplitude or phase that may occur in the channel. There are various types of large-scale propagation path loss models, i.e. deterministic, stochastic and empirical.

Ray tracing is a technique commonly used in deterministic models, very popular and accurate for simulating the nature of the wireless channel, due to the fact that reflections and dispersions are predominant in millimeter-wave and that the diffraction contributions are scarce. Geometrical optics is used in ray tracing to simulate the propagation of the wave through the medium.

The main limitations of the models based on ray tracing are their poor flexibility, since they are only applicable to a specific scenario - it is almost necessary to know all the characteristics of the surrounding materials for each case - and their high computational cost. Thus, these channel models (indoor or outdoor) are difficult to use if there is not enough information about the propagation scenario. In most of the cases, it is convenient to limit the number of reflections (reducing in that way the computational cost), since good results can be obtained with few ones.

Regarding stochastic models, the log-normal distribution is the most used for describing the large-scale fading. The fluctuations of the field-strength averaged at small scale can be plotted on a logarithmic scale, showing a Gaussian distribution around a mean $\mu$. A useful property of this distribution is that the sum of log-normally distributed values is also lognormally distributed, approximately $[\mathbf{1 9 2}]$.

Empirical or semi-empirical models are the most utilized in wireless communications, since they are based on experimental measurements (hence they provide a realistic insight on the propagation channel) and their results can be extrapolated to other conditions. Models with few parameters, an intuitive physical explanation, adequate closed form equations and 
with parameters confirmed by a large amount of data are preferable to those that may be more complicated, deviate from propagation physical principles and have high variability for different data at similar scenarios [164].

Despite the variety of models, the aim is to have only one path loss model per scenario in LOS and NLOS configurations. A comprehensive analysis of large-scale path loss models analysis for different propagation scenarios is provided in [174] and its reading is recommended for the cases not addressed here. In this Thesis, only the main specifications for $\mathrm{InH}$ are given in depth, since only measurements in such scenario have been taken.

The three more commonly used large-scale path loss model types are presented in the next sub-sections, and different variants are included for some of them. They use linear regression techniques to derive coefficients for certain conditions, which may be proposed later as model coefficients. Then, they can also be considered as fitting models.

\subsubsection{CI (CIF, CIX, CIFX) Model}

The Close-In free space reference distance (CI) path loss model estimates the path loss as $[\mathbf{1 8 2}]$ :

$$
P L^{C I}(d)[\mathrm{dB}]=L\left(d_{0}\right)+10 \cdot n \cdot \log \left(\frac{d}{d_{0}}\right)+\chi_{\sigma}^{C I}
$$

where:

$$
\begin{aligned}
& d_{0}(m): \text { Reference distance in free space, usually } 1 \mathrm{~m} . \\
& d(m) \quad: \text { Distance between the transmitter and the receiver, longer than } 1 \mathrm{~m} . \\
& n \quad: \text { Path loss exponent (PLE) that describes the dependence of propagation }\text { loss in the path (in } \mathrm{dB}) \text { to the logarithm of the distance starting at } d_{0} . \\
& \chi_{\sigma}^{C I} \quad: \text { Gaussian random variable with zero mean and standard deviation } \sigma(\mathrm{dB}), \text { that characterizes the large-scale channel fluctuations due to shadowing. } \\
& L\left(d_{0}\right) \quad: \text { Free space loss at a distance } d_{0}=1 \mathrm{~m}, \text { with } f_{c} \text { being the center frequency: } \\
& \qquad L_{0}(d B)=20 \log \left(\frac{4 \pi d_{0}}{\lambda}\right)=32.4+20 \log f_{c}(\mathrm{GHz})
\end{aligned}
$$

This term is also known as the path loss intercept.

This model is also referred as power law model $[\mathbf{1 6 3}]$ and the path loss is a function of the logarithm of the distance and a path loss exponent $n(n=2$ describes the free space propagation and $n=4$ expresses the two ray channel model, direct and reflected ray, for long distances) which is the only parameter to adjust via a linear regression over all the measured PL data. Also, it has an intrinsic dependence on the frequency in $L\left(d_{0}\right)$.

The CI model can be used to estimate the path loss from measurements with any polarization of the signal and for several frequencies with a good degree of accuracy for outdoor channels, but for indoor channels the addition of other parameter depending on the polarization gives better results [194]. Doing so, the Close-In reference distance with XPD 
(CIX) model is obtained, in which an optimized constant attenuation for cross polar discrimination (XPD) is added:

$$
P L^{C I X}(f, d)[\mathrm{dB}]=L\left(d_{0}\right)+10 \cdot n_{(V-V)} \cdot \log \left(\frac{d}{d_{0}}\right)+X P D+\chi_{\sigma}^{C I X}
$$

with:

$n_{(V-V)}$ : Polarization path loss exponent, determined by measurements (in this equation a Vertical to Vertical polarization configuration $\mathrm{V}-\mathrm{V}$ is used, although other combinations are also possible such as the Horizontal to Horizontal H-H and the hybrid case V-H).

XPD : Optimized cross polar polarization attenuation factor, in $\mathrm{dB}$.

$\chi_{\sigma}^{C I X}:$ Zero mean Gaussian (in $\mathrm{dB}$ ) shadow fading random variable that describes the large-scale variation of the signal power about the mean path loss and the constant XPD-attenuation term, as a function of distance.

The XPD value is estimated by using the co-polarization PLE of (3.5) to determine the best fit XPD factor caused by antenna polarization mismatch. The CIX model uses the PLE (as a constant) found from co-polarized antenna measurements at identical location of crosspolarized measurements and adds this optimized constant $[\mathbf{1 8 2}]$.

A multi-frequency approach can be considered - very adequate, since indoor environments have frequency-dependent loss beyond the first meter due to the surrounding environment $[\mathbf{1 7 4}]$ - , having then a Close-In free space reference distance with Frequency dependent path loss exponent (CIF), that uses the same physically motivated free space path loss anchor at $1 \mathrm{~m}$ as the CI model. The model is now:

$$
P L^{C I F}(f, d)[\mathrm{dB}]=L\left(f, d_{0}\right)+10 \cdot n\left(1+b\left(\frac{f-f_{0}}{f_{0}}\right)\right) \log \left(\frac{d}{d_{0}}\right)+\chi_{\sigma}^{C I F}
$$

where:

$f(G H z)$ : Carrier frequency in GHz.

$f_{0}(G H z)$ : An average reference frequency that serves as the balancing point or center for the linear frequency dependence of PLE and is based on the weighted average of all frequencies represented by the model. It is calculated using information of the measurements set:

$$
f_{0}=\frac{\sum_{k=1}^{K} f_{k} N_{k}}{\sum_{k=1}^{K} N_{k}}
$$

Where $K$ is the number of unique frequencies, $N_{k}$ is the number of path loss data points corresponding to the $k$-th frequency $f_{k}$. Usually, $f_{0}$ is rounded to the nearest integer in GHz.

Other approach is assuming $f_{0}$ as the minimum investigated frequency [185]. 
$b$ : Optimization parameter that represents the path loss slope of the linear frequency dependence.

$\chi_{\sigma}^{C I F}$ : Zero mean Gaussian random variable (in $\mathrm{dB}$ ) that describes the largescale signal fluctuations about the mean path loss over distance.

If the two previous factors are taken into account, a more general CI model for multiple frequencies that takes into consideration the polarization is obtained:

$$
P L^{C I F X}(f, d)[\mathrm{dB}]=L\left(f, d_{0}\right)+10 \cdot n\left(1+b\left(\frac{f-f_{0}}{f_{0}}\right)\right) \log \left(\frac{d}{d_{0}}\right)+X P D[d B]+\chi_{\sigma}^{C I F}
$$

For InH, a dual-slope path loss model might provide a good fit for different distance zones in the propagation environment [180]. The dual-slope may be best suited for InH shopping malls or long indoor distances (longer than $50 \mathrm{~m}$ ). The dual-slope CIF path loss model for InH scenarios (it can be seen also as a generalization of the previously described CIF model) is:

$$
P L_{\text {Dual }}^{\text {CIF }}(d)[\mathrm{dB}]=\left\{\begin{array}{cc}
L\left(f, d_{0}\right)+10 \cdot n_{1}\left(1+b_{1}\left(\frac{f-f_{0}}{f_{0}}\right)\right) \log \left(\frac{d}{d_{0}}\right) & 1<d \leq d_{B P} \\
L\left(f, d_{0}\right)+10 \cdot n_{1}\left(1+b_{1}\left(\frac{f-f_{0}}{f_{0}}\right)\right) \log \left(\frac{d_{B P}}{d_{0}}\right) & d>d_{B P} \\
+10 \cdot n_{2}\left(1+b_{2}\left(\frac{f-f_{0}}{f_{0}}\right)\right) \log \left(\frac{d}{d_{B P}}\right) &
\end{array}\right.
$$

$d_{B P}$ being a LOS breakpoint distance in the path loss function.

The criterion for finding $d_{B P}$ is to minimize the global standard deviation of the shadow fading by setting iteratively (in an ascendant way) all the possible distances as the breakpoint, calculating the two slopes and the correspondent segments (given by (3.9)), checking the resultant RMS for each assigned distance, and finally choosing the breakpoint distance corresponding to the minimum RMS [195].

\subsection{2. $\quad$ FI Model}

The Floating-Intercept (FI) path loss model requires two parameters, as (3.10) shows:

$$
P L^{F I}(d)[\mathrm{dB}]=\alpha+10 \cdot \beta \cdot \log d+\chi_{\sigma}^{F I}
$$

where:

$d(m)$ : Distance between the transmitter and receiver, longer than or equal to $1 \mathrm{~m}$.

$\chi_{\sigma}^{F I} \quad$ : Shadow fading log-normal random variable which describes the large-scale signal fluctuations about the mean path loss over the distance with mean 0 $\mathrm{dB}$ and standard deviation $\sigma(\mathrm{dB})$. 
$\beta \quad$ : Slope of the line that characterizes the loss distance dependence.

$\alpha \quad$ : Floating-intercept in $\mathrm{dB}$ without a physical meaning, used to adjust the measurements.

This model is used in the WINNER II and 3GPP channel models. CI and FI path loss models produce very similar shadow fading standard deviations in outdoor millimeter-wave channels, then it is doubtful that using an extra modeling parameter in that case produces an improvement in the modeling when there is a lack of physical relationship to the transmitted power [182].

\subsubsection{ABG Model}

This is a multi-frequency type model, and is intended to model the long-scale propagation loss with a function that depends on the frequency and the distance:

$$
P L^{A B G}(f, d)[\mathrm{dB}]=\alpha+10 \cdot \beta \cdot \log \left(\frac{d}{1 \mathrm{~m}}\right)+10 \cdot \gamma \cdot \log \left(\frac{f}{1 \mathrm{GHz}}\right)+\chi_{\sigma}^{A B G}
$$

where:

$\alpha \quad$ : Optimized offset parameter without a physical meaning.

$\beta$ : Coefficient that describes the slope of the path loss with the logarithm of the distance.

$\gamma$ : Coefficient that describes the path loss frequency dependence.

$\chi_{\sigma}^{A B G} \quad$ : Gaussian random variable representing the shadowing of the large-scale signal fluctuations about the mean path loss over the distance.

The ABG model is an extension of the FI model for multiple frequencies, and the FI can be obtained (when setting $\gamma=0$ or 2) if a single frequency is used [182]. Also, it can be reduced to the CI if $\alpha$ is equal to $20 \log (4 \pi / c), \beta$ to the PLE $n$ and $\gamma$ to 2 .

If a polarization factor XPD (in $\mathrm{dB}$ ) is considered in the case of cross polarized propagation, then:

$$
P L^{A B G X}(f, d)[\mathrm{dB}]=\alpha+10 \beta \cdot \log \left(\frac{d}{1 \mathrm{~m}}\right)+10 \gamma \cdot \log \left(\frac{f}{1 \mathrm{GHz}}\right)+X P D[\mathrm{~dB}]+\chi_{\sigma}^{A B G X}
$$

The ABG and CIF are statistical multi-frequency models (hence stochastic) [183] that describe the large-scale path loss over distance and frequency for a given scenario. It can be noticed that the CI and the CIF models are very similar to the FI model; in the first two the FI floating-constant is equal to the free space path loss at $1 \mathrm{~m}$.

As in the case of the CIF model, the ABG model might provide a good fit for different distance zones with a dual-slope variation. Then, the dual-slope ABG model is: 


$$
P L_{\text {Dual }}^{A B G}(d)[\mathrm{dB}]=\left\{\begin{array}{cc}
\alpha_{1}+10 \cdot \beta_{1} \cdot \log (d)+10 \cdot \gamma \cdot \log (f) & 1<d \leq d_{B P} \\
\alpha_{1}+10 \cdot \beta_{1} \cdot \log \left(d_{B P}\right)+10 \cdot \gamma \cdot \log (f) & d>d_{B P} \\
+10 \cdot \beta_{2} \cdot \log \left(\frac{d}{d_{B P}}\right) &
\end{array}\right.
$$

The criterion for finding $d_{B P}$ is the same presented above for the CIF dual slope case, but using now the expression of (3.13).

\subsection{Path loss models for Indoor scenarios}

Walls, roof-tops, cubicle material, furniture, windows and metal structures have a great impact in the propagation at millimeter-wave frequencies in indoor scenarios. Besides, these structures can be located in such ways that mitigate the interference between users; a reason to have measurements of several kind of scenarios for modeling accurately the propagation.

Indoor propagation degradations can be mainly caused by [196]:

- Reflections in the objects (including walls and floor) and diffraction around them inside the room.

- Transmission loss through walls, floors and other obstacles.

- Channeling of energy, especially in halls for higher frequencies.

- Movements of people and objects in the room, including possible one or both ends of the radio link.

These degradations are:

- Path loss, including not only the free space path loss but also additional losses due to obstruction and transmission through materials.

- Variation in the path loss in time and space.

- Multipath effects from multiple wave reflections and diffractions.

- Depolarization caused by the random alignment of the receiver.

In the literature, there are several path loss models for indoor propagation at microwave frequencies, an outline of the more relevant ones can be found in [163]. Some recent and specific models that can handle indoor scenarios and were developed for supporting millimeter-wave propagation are related next.

\subsubsection{METIS models}

From the analyses carried out in the METIS project [166] it was concluded that the analyzed models (developed before 2015) did not describe adequately the propagation at frequencies higher than those used in the present wireless systems - specifically, those in the 6 to $60 \mathrm{GHz}$ range - and that around $60 \mathrm{GHz}$ there are only few available models for very limited scenarios $[\mathbf{1 7 6}]$. 
This project proposed three channel models to be used either in outdoor environments or in indoor scenarios: the map-based model, the stochastic model and the hybrid model. Each one has different scenario classifications; however in all of them the indoor scenarios are classified as Office and Shopping-Mall. For the three, their main characteristics in indoor propagation scenarios will be addressed in the following (the hybrid model can be applied in the same scenarios and frequencies as the stochastic model, a reason to not consider it separately). A comparison between the METIS models is given in Table 3.2.

Table 3.2. Comparison between METIS Models [176]

\begin{tabular}{|c|c|c|c|}
\hline \multirow{2}{*}{ Feature } & \multicolumn{3}{|c|}{ METIS Model } \\
\hline & Map-based & Stochastic & Hybrid \\
\hline Frequency range & Up to $100 \mathrm{GHz}$ & Up to $70 \mathrm{GHz}$ & Up to $70 \mathrm{GHz}$ \\
\hline Valid Bandwidth & $\begin{array}{l}10 \% \text { of the } \\
\text { center frequency }\end{array}$ & $\begin{array}{c}100 \mathrm{MHz}<6 \mathrm{GHz}, \\
1 \mathrm{GHz} @ 60 \mathrm{GHz}\end{array}$ & $\begin{array}{c}100 \mathrm{MHz}<6 \mathrm{GHz}, \\
1 \mathrm{GHz} @ 60 \mathrm{GHz}\end{array}$ \\
\hline Path loss & implicit & separate, empirical & implicit \\
\hline Shadowing & implicit & separate & implicit \\
\hline Explicit/generic building model & explicit & generic & explicit \\
\hline Parameterization by measurements & easy & easy & easy \\
\hline Support massive-MIMO & yes & limited & limited \\
\hline Support spherical waves & yes & no $*$ & no $*$ \\
\hline $\begin{array}{l}\text { Support extremely large arrays } \\
\text { beyond stationary interval }\end{array}$ & yes & no & no \\
\hline Support dual mobility & yes & limited** & limited** \\
\hline Support 3D & yes & yes & yes \\
\hline Support millimeter-waves & yes & partial & partial \\
\hline Dynamic modeling & yes & no & no \\
\hline Polarization modeling & ray-based & XPR & XPR \\
\hline Maturity & medium & high & medium \\
\hline Complexity in terms of definition & high $* * *$ & medium & high \\
\hline $\begin{array}{l}\text { Complexity in terms of calculation } \\
\text { of channel realizations }\end{array}$ & $\operatorname{high}^{* * *}$ & medium & medium-high \\
\hline Public implementation available & no & no & no \\
\hline \multicolumn{4}{|l|}{ XPR : Cross polarization ratio } \\
\hline \multicolumn{4}{|c|}{ * $\quad$ Possible, if the physical cluster location is fixed. } \\
\hline \multicolumn{4}{|c|}{ ** Spatially consistent shadowing, AoA/AoD/Doppler. } \\
\hline \multicolumn{4}{|c|}{ Simpler than a full-blown ray tracing. Simplifications can be applied. } \\
\hline
\end{tabular}




\subsubsection{Map-based model}

This model can be used for frequencies between 0.45 and $100 \mathrm{GHz}$ and in cases where accurate and realistic spatial channel features -as in the case of massive MIMO and beamforming - are required. The model is based on ray tracing using a 3-D geometrical description of the environment. It takes into account the contributions of different phenomena such as diffraction, specular reflection, diffuse scattering and propagation obstruction. The complexity can be scaled; hence the model can be simplified if some reflections, scattered paths and dispersions are not taken into consideration or if a simplified diffraction model is used. Some parameters for indoor configuration are provided in Table 3.3 .

Table 3.3. Parameters for the map-based model [166]

\begin{tabular}{lccc}
\hline \hline \multirow{2}{*}{ Parameter } & Symbol & \multicolumn{2}{c}{ Indoor propagation scenarios } \\
\cline { 3 - 4 } & {$[$ unit $]$} & Office & Shopping mall \\
\hline Object density & $\mathrm{D}\left[1 / \mathrm{m}^{2}\right]$ & 0.05 & 0.05 \\
Object height & $\mathrm{h}[\mathrm{m}]$ & 1.5 & 1.5 \\
Object width & $\mathrm{w}[\mathrm{m}]$ & 0.5 & 0.5 \\
Scatterer absorption coefficient & $\alpha$ & 0 & 0 \\
Specular/diffuse power ratio & $\beta$ & 0.5 & 0.5 \\
Angle dependency factor & $q_{\lambda}\left[\mathrm{m}^{-1}\right]$ & 0.031 & 0.031 \\
Angle dependency exponent & $\nu$ & 3.5 & 3.5 \\
Angle dependency factor & $\gamma$ & 0.95 & 0.95 \\
\hline \hline
\end{tabular}

The step-by-step procedure can be found in [166]. An upgrade of the map-based model was provided in [7], where some modifications were introduced in the resulting transfer function.

\subsubsection{Stochastic and hybrid models}

For a given scenario, the propagation can be estimated in a deterministic way if there are available characteristics of furniture and/or other surrounding objects. However, if that information is not available the propagation modeling along the path can be generated in a stochastic manner. Moreover, it is possible to use explicit information of the propagation scenarios in a stochastic model with the objective of improving the results for a given location, naming that approach as hybrid modeling. The stochastic model is based on a geometry-based stochastic channel model (GSCM). As it was developed from the WINNER/3GPP model [197], its use in millimeter-wave is limited to some model specifications and recommended only for frequencies below $6 \mathrm{GHz}$ and in the $60-\mathrm{GHz}$ band.

The METIS stochastic model proposes the following equations to describe the path loss for Shopping-Mall InH LOS and NLOS scenarios for a limited millimeter wave range.

$$
P L_{L O S}=68.8+18.4 \log \left(d_{2 D}\right) \quad 1.5<d_{2 D}<13.4 \mathrm{~m} \quad f_{c}=50-70 \mathrm{GHz}
$$




$$
\begin{aligned}
& P L_{N L O S}=94.3+3.59 \log \left(d_{2 D}\right) \quad 4<d_{2 D}<16.1 \mathrm{~m} \quad \sigma_{S F}=2.0 \\
& h_{B S}=h_{U E}=2 \mathrm{~m}
\end{aligned}
$$

\subsubsection{GPP TR 38.901 model}

The work carried out by the 3GPP for modeling the 5G channels [188] [189] can be identified as an ongoing effort and may serve in the standardization of $5 \mathrm{G}$. It relates a large number of geometrical parameters - that can be found in the previous references-, covering from the arrival angles of the last bounce scatterers and their respective departure angles to the first interaction with the scatterers in the transmitting side.

The path loss equations presented in the 3GPP 5G model for indoor scenarios have a CItype equation for LOS and NLOS and additionally an FI-type expression for NLOS:

$$
\begin{aligned}
& P L_{L O S}=32.4+17.3 \log \left(d_{3 D}\right)+20 \log \left(f_{c}\right) \quad \sigma_{S F}=3.0 \mathrm{~dB} \quad 1 \mathrm{~m} \leq d_{3 D} \leq 150 \mathrm{~m} \\
& P L_{N L O S}=\max \left(P L_{L O S}, P L^{\prime}{ }_{N L O S}\right) \quad \sigma_{S F}=8.03 \mathrm{~dB} \quad 0.5<f_{c}<100 \mathrm{GHz} \\
& P L^{\prime}{ }_{N L O S}=17.3+38.3 \cdot \log \left(d_{3 D}\right)+24.9 \log \left(f_{c}\right)
\end{aligned}
$$

\section{(Optional)}

$$
P L^{\prime}{ }_{N L O S}=32.4+31.9 \cdot \log \left(d_{3 D}\right)+20 \log \left(f_{c}\right) \quad \sigma_{S F}=8.29 \mathrm{~dB}
$$

The channel model generated for low complexity and earlier evaluations without additional modeling components (such as oxygen absorption, large bandwidth and large antenna arrays, spatial consistency, blockage, multi-frequency simulations, time-varying Doppler shift, user terminal (UT) rotation and ground reflection) can be found in [188].

The aforementioned additional modeling components can be turn-on (affecting some model steps) in order to support advanced simulations, such as those related with very large arrays and large bandwidth, simulations affected by oxygen absorption (frequencies between 53 and $67 \mathrm{GHz}$ ), simulations in which spatial consistency is important (e.g. high number of closely located user), simulations of mobility, and simulations with blockage effects. These extensions are computationally expensive and might not be required in all evaluation cases.

\subsubsection{GCM model}

The contribution of the $5 \mathrm{GCM}$ to the omnidirectional path loss modeling in indoor scenarios was presented in [180]. In LOS conditions, multiple reflections from walls, floor and ceiling produce a waveguide-like propagation effect. The measurements in both Office and Shopping-Mall scenarios show that PLEs, based on a 1-m free space reference distance, are typically below two, indicating a path loss more favorable than the predicted by the Friis' free space path loss equation. The strength of the wave-guiding effect is variable and the PLE appears to increase very slightly when frequency increases, possibly due to the relation between the wavelength and the surface roughness.

The 5GCM model shares some steps with the previous 3GPP model. Actually, in [180] the 12-step procedure of the $3 \mathrm{GPP} 5 \mathrm{G}$ channel model is proposed as a good starting point 
and from there some modifications are performed and new steps are added to comply with the 5GCM proposal.

For the general path loss models (specifically for the CI, CIF and ABG), it is worth mentioning that in $[\mathbf{1 8 2}]$ it was concluded that in indoor channels an increase in the PLE value is observed if the frequency increases. Besides, cross-polarization and closed-form equations for optimization in indoor channels can be consulted in that work.

Some parameters for the aforementioned general path loss models are shown in Table 3.4.

Table 3.4. CI, CIF and ABG model parameters for InH environments [180]

\begin{tabular}{lcc}
\hline \hline Scenario & CI/CIF Model Parameters & ABG Model Parameters \\
\hline Office-LOS & $n=1.73, \sigma_{S F}=3.02 \mathrm{~dB}$ & $\mathrm{NA}$ \\
Office-NLOS single & $n=3.19 ; b=0.06 ; f_{0}=24.2 \mathrm{GHz} ;$ & $\alpha=17.3 ; \beta=3.83 ; \gamma=2.49 ;$ \\
slope & $\sigma_{S F}=8.29 \mathrm{~dB}$ & $\sigma_{S F}=8.03 \mathrm{~dB}$ \\
Office-NLOS dual slope & $n_{1}=2.51 ; b_{1}=0.12 ; f_{0}=24.1 \mathrm{GHz} ;$ & $\alpha_{1}=33.0 ; \beta_{1}=1.7 ; \gamma=2.49 ;$ \\
& $n_{2}=4.25 ; b_{2}=0.04 ; d_{B P}=7.8 \mathrm{~m} ;$ & $d_{B P}=6.9 \mathrm{~m} ; \beta_{2}=4.17 ;$ \\
& $\sigma_{S F}=7.65 \mathrm{~dB}$ & $\sigma_{S F}=7.78 \mathrm{~dB}$ \\
Shopping Malls-LOS & $n=1.73 ; \sigma_{S F}=2.01 \mathrm{~dB}$ & $\mathrm{NA}$ \\
Shopping Malls-NLOS & $n=2.59 ; b=0.01 ; f_{0}=39.5 \mathrm{GHz} ;$ & $\alpha=18.09 ; \beta=3.21 ; \gamma=2.24 ;$ \\
single slope & $\sigma_{S F}=7.40 \mathrm{~dB}$ & $\sigma_{S F}=6.97 \mathrm{~dB}$ \\
Shopping Malls-NLOS & $n_{1}=2.43 ; b_{1}=-0.01 ;$ & $\alpha_{1}=22.17 ; \beta_{1}=2.9 ; \gamma=2.24 ;$ \\
dual slope & $f_{0}=39.5 \mathrm{GHz} ; n_{2}=8.36 ; b_{2}=0.39 ;$ & $d_{B P}=147.0 \mathrm{~m} ; \beta_{2}=11.47 ;$ \\
& $d_{B P}=110 \mathrm{~m} ; \sigma_{S F}=6.26 \mathrm{~dB}$ & $\sigma_{S F}=6.36 \mathrm{~dB}$
\end{tabular}

The applicability range of the previous formulae goes for frequencies from $6 \mathrm{GHz}$ to $100 \mathrm{GHz}$

For NLOS scenarios, the dual-slope ABG and the dual-slope CIF models are considered in the $5 \mathrm{G}$ performance evaluation [174], with more parameters to be optimized than the single-slope CIF. The dual-slope model could be best suited for InH-Shopping Mall or long indoor distances (longer than $50 \mathrm{~m}$ ), although it is not completely proved that an increase in complexity is enough guarantee for better approximations when comparing with the singleslope CIF model results.

Some interesting issues of the 5GCM model for indoor scenarios are the shadowing and the building penetration loss modeling. From the shadowing study, it was found that in LOS condition, the frequency and distance dependency is weak, but in the NLOS case, frequency and distance dependency can be observed [180]. For the building penetration, a loss value of $0.5 \mathrm{~dB} / \mathrm{m}$ has been selected to maintain the consistency with the 3GPP LTE model [198].

\subsection{4. mmMAGIC model}

The mmMAGIC is a GSCM channel model, comprising in its baseline components of the 3GPP and QuaDRiGa models and additional features that extend its accuracy and applicability [190]. Highlighted features are the addition of the ground reflection effects, blockage, O2I (Outdoor to Indoor) building penetration loss modeling, the support of large bandwidths and antenna arrays, and the provision of spatial consistency. Some mmMAGIC features are implemented in QuaDRiGa v2.0, available as open-source software in [199]. In 
the development of this model [190] there was an active contribution of the mmMAGIC partners $^{5}$ within the $3 \mathrm{GPP}$ and the ITU-R working groups.

The mmMAGIC model is focused on the 6-100 GHz range, but it is applicable down to 2 $\mathrm{GHz}$ since the measurements and evaluations were extended to such lower frequencies. The model supports a bandwidth of up to $10 \%$ of the center frequency but no larger than $2 \mathrm{GHz}$. An overview of the model components can be consulted in [190].

For indoor scenarios, mmMAGIC gives the following equations for the LOS and NLOS cases:

$$
\begin{array}{cc}
P L_{L O S}=33.6+13.8 \log \left(d_{3 D}\right)+20.3 \log \left(f_{c}\right) & \sigma_{S F}=1.18 \mathrm{~dB} \\
P L_{N L O S}=\max \left(P L_{L O S}, P L^{\prime}{ }_{N L O S}\right) & \sigma_{S F}=8.03 \mathrm{~dB} \\
\text { with: } & \\
P L^{\prime}{ }_{N L O S}=15.2+36.9 \log \left(d_{3 D}\right)+26.8 \log \left(f_{c}\right) &
\end{array}
$$

\subsubsection{ITU-R Rec. P.1238-9 Model}

This ITU-R Recommendation [196] gives information of indoor propagation for frequencies from $300 \mathrm{MHz}$ to $1000 \mathrm{GHz}$ regarding to: path loss, delay dispersion, angular dispersion, effects of the antenna pattern diagram and polarization, effects of the transmitter and receiver locations, effects of the construction materials, furniture, object movements in the room and a statistical model in case of statistical use. Moreover, some classifications for indoor propagation are given, specifying the main parameters and their characteristics.

The InH path loss is characterized by a mean path loss and statistics associated with fades due to the shadowing. The path loss equation for a several-floor scenario is:

$$
L_{\text {total }}(d B)=L\left(d_{0}\right)+N \cdot \log \left(\frac{d}{d_{0}}\right)+L_{f}(\text { floors })
$$

where:

$N$ : Distance dependent power loss coefficient (analogous to the PLE but divided by ten, several values are given for different indoor environments in $[186])$.

$d$ : Separation distance (in $\mathrm{m})$ between the transmitter and receiver $(d>1 \mathrm{~m})$.

$d_{0}$ : Reference distance (in $\mathrm{m}$ ).

$f \quad$ : Frequency (in $\mathrm{MHz}$ ).

${ }^{5}$ mmMAGIC project was co-funded by the European Commission's 5G PPP program, with the interaction of major infrastructure vendors (Samsung, Ericsson, Alcatel-Lucent, Huawei, Intel and Nokia), major European operators (Orange and Telefónica), research institutes and universities (Fraunhofer HHI, CEA Leti, IMDEA Networks, Universities of Aalto, Bristol, Chalmers and Dresden), measurement equipment vendors (Keysight Technologies and Rohde \& Schwarz) and one small and medium enterprise (Qamcom). 
$L\left(d_{0}\right) \quad$ : Free space path loss for $d_{0}$. When $d_{0}=1 \mathrm{~m}$ :

$$
L\left(d_{0}\right)=20 \log f-28
$$

$L_{f} \quad$ : Loss factor due to floor penetration (in $\mathrm{dB}$ ).

floors : Number of floors between the base station and the receiver $(n \geq 0)$, with $L_{f}=0 d B$ for $n=0$.

This model is a CI-type if it is used without a floor penetration factor. Also, it is considered site-general and requires little information about the path or the location.

\subsection{Multiple-Input Multiple-Output (MIMO) fundamentals}

Due to the MIMO potentialities to simultaneously transmit several streams of high-rate data, it is expected that this technology will play a paramount role in the $5 \mathrm{G}$ systems. MIMO communication systems can be described as those in which the transmitter and receiver are equipped with multiple antennas, producing a multi-path richness, which is exploited in the communication benefit by multiplying the channel capacity without need of more spectrum, by means of $[\mathbf{1 9 1}][\mathbf{2 0 0}]$ :

- Array gain, that leads to an increase of the average received Signal-to-Noise Ratio (SNR) by processing the signals at transmitter and receiver sides and their coherent combination.

- Diversity gain, obtained from the de-correlation of the channels between the different antennas. The effects of random fluctuations of the signal power can be mitigated by transmitting the signals over multiple de-correlated channels.

- Spatial multiplexing gain, allowing an increase in the capacity by sending multiple data streams simultaneously and through channels separated in space. Also, the capacity in the MIMO channels with a given $n_{t}$ transmit and $n_{r}$ receive antennas is proportional to the minimum number of $n_{t}$ and $n_{r}$.

- Interference reduction and avoidance, interference may be mitigated by exploiting the spatial dimension to increase the separation between users. The spatial dimension may serve also for the interference avoidance by directing more efficiently the signal energy towards the intended user and minimizing interference to others.

All these benefits may not be possible to exploit simultaneously due to conflicting demands, but a combination of some of them will improve capacity, coverage and reliability [200].

A large number of books addressed MIMO topic [200] [201] [202] [203], but here only the basic mathematical aspects necessary for a first understanding are given. The limit is drawn by the limitations imposed by the available experimental setup. 
The deterministic $\mathbf{H}_{\boldsymbol{n}_{\boldsymbol{r}} \times \boldsymbol{n}_{\boldsymbol{t}}}$ matrix is commonly used to describe narrow-band timeinvariant wireless channel with $n_{t}$ transmit and $n_{r}$ receive antennas [191]. The individual time-invariant channels can be described as:

$$
\mathbf{y}=\mathbf{H} \cdot \mathbf{x}+\mathbf{w}
$$

with $\mathbf{x} \in \mathbb{C}^{n_{t}}, \mathbf{y} \in \mathbb{C}^{n_{r}}$ and $\mathbf{w} \sim \mathbb{C} N^{n_{r}}\left(0, N_{0} \mathbf{I}_{n_{r}}\right)$ being the transmitted signal, the received signal and a white Gaussian noise vector, respectively. The channel matrix $\mathbf{H} \in \mathbb{C}^{n_{r} \times n_{t}}$ is deterministic and assumed to be constant at all times and known to both transmitter and receiver. The matrix elements $h_{i j}$ are the channel gains from transmit antenna $j$ to receive antenna $i$.

The capacity can be obtained by decomposing the vector channel into a set of parallel independent scalar Gaussian sub-channels. This linear transformation is done by applying three operations: a rotation, a scaling and another rotation. Then, the matrix $\mathbf{H}$ can be described by a singular value decomposition (SVD) as:

$$
\mathbf{H}=\mathbf{U} \boldsymbol{\Lambda} \mathbf{V}^{*}
$$

where $\mathbf{U} \in \mathbb{C}^{n_{r} \times n_{t}}$ and $\mathbf{V} \in \mathbb{C}^{n_{r} \times n_{t}}$ are unitary matrices (that represent rotations), $\boldsymbol{\Lambda} \in \mathbb{R}^{n_{r} \times n_{t}}$ is a rectangular matrix whose diagonal elements are non-negative real numbers and whose non-diagonal elements are zero and $\left(^{*}\right)$ being the symbol of the conjugate transpose of the given matrix. The ordered diagonal elements of $\boldsymbol{\Lambda}\left(\lambda_{1} \geq \lambda_{2} \geq \cdots \geq \lambda_{n_{\text {min }}}\right.$, with $n_{\text {min }}=\min \left(n_{t}, n_{r}\right)$ being the number of singular values) are named as singular values of H.

Having:

$$
\mathbf{H H}^{*}=\mathbf{U} \boldsymbol{\Lambda} \boldsymbol{\Lambda}^{\mathrm{t}} \mathbf{U}^{*}
$$

the matrix $\mathbf{H H}^{*}$ (as well as the $\mathbf{H}^{*} \mathbf{H}$ matrix) has $\lambda_{i}^{2}$ eigenvalues.

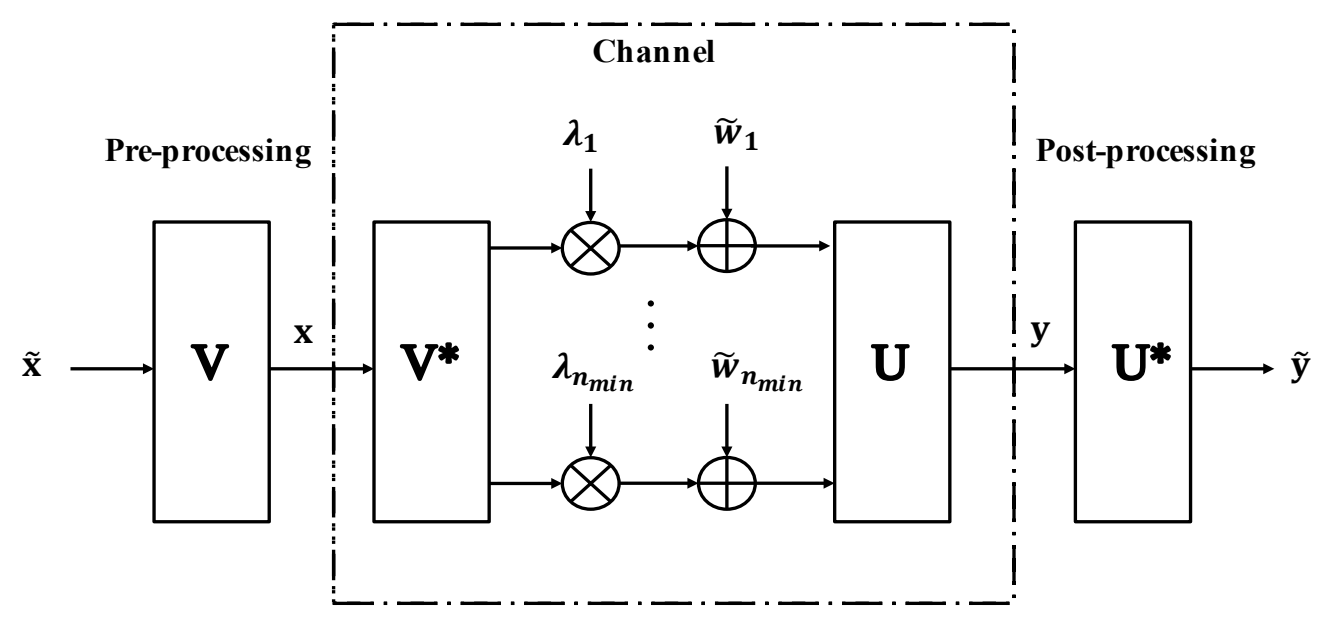

Figure 3.1. Conversion of the MIMO channel into a parallel channel using SVD [191].

Following the MIMO channel configuration of Figure 3.1, the following relations are defined:

$$
\tilde{\mathbf{x}}=\mathbf{V}^{*} \mathbf{x}
$$




$$
\begin{aligned}
\tilde{\mathbf{y}} & =\mathbf{U}^{*} \mathbf{y} \\
\widetilde{\mathbf{w}} & =\mathbf{U}^{*} \mathbf{w}
\end{aligned}
$$

Being possible to rewrite (3.21) as:

$$
\tilde{\mathbf{y}}=\boldsymbol{\Lambda} \cdot \tilde{\mathbf{x}}+\widetilde{\mathbf{w}}
$$

In MIMO measurements, the capacity of the channel is one of the main parameters for determining the performance. The channel capacity can be approximated as:

$$
C \approx \sum_{i=1}^{k} \log _{2}\left(1+\frac{P}{k N_{0}} \lambda_{i}^{2}\right)
$$

With $k$ being the number of non-zero $\lambda_{i}^{2}$ spatial degrees of freedom per second per Hertz (the rank of the matrix $\mathbf{H}$ ). The channel capacity is evaluated here only by means of the rank and the condition number (a first-order variable that determines the capacity of the channel $[\mathbf{1 9 1}])$. The non-zero singular values can be derived from the following inequality:

$$
\frac{1}{k} \sum_{i=1}^{k} \log _{2}\left(1+\frac{P}{k N_{0}} \lambda_{i}^{2}\right) \leq \log _{2}\left(1+\frac{P}{k N_{0}}\left(\frac{1}{k} \sum_{i=1}^{k} \lambda_{i}^{2}\right)\right)
$$

The total power gain of the channel matrix, if the energy is equally spread between all the transmitter antennas, can be interpreted as:

$$
\sum_{i=1}^{k} \lambda_{i}^{2}=\operatorname{Tr}\left[\mathbf{H H}^{*}\right]=\sum_{i, j}\left|h_{i j}\right|^{2}
$$

with $\operatorname{Tr}[\mathrm{X}]$ being the trace of the square matrix $\mathrm{X}$.

Then, it can be said that from the channels with equally total power gain, the one with the highest capacity is the one with all the singular values being equal: in the high SNR regime, the less spread out the singular values, the larger the capacity [191]. Here, the analysis is focused in high SNR regimes since the measurements are taken at relatively short distances with a high probability of high SNR.

The condition number of $\mathbf{H}$ is $\left(\max _{i} \lambda_{i} / \min _{i} \lambda_{i}\right)$ and a well-conditioned matrix (a condition number close to 1) maximizes the capacity of communication in high SNR regimes.

\subsection{Conclusions}

Millimeter-wave channel modeling is a topic in which much work has been carried in the last few years by several research groups. The future use of millimeter-wave frequencies for 5G has led this trend. Of a particular interest is the indoor modeling, since most cellular communications take place in this scenario.

The developed models rely on some general large-scale path loss models (or fittings) such as the CI, the FI and the ABG (and their variants). In general, the path loss equations for models either for LOS or NLOS condition, serve for a wide range of frequencies. Nevertheless, differences may arise between predictions and measurements due to the differences between 
the scenarios used for the model development and the actual scenario. Although most of the models give path loss equations for several scenarios, the amount of measurements used to derive them is not so large and in some frequencies of interest no measurements have been taken. Then, the collection of millimeter-wave measurements for path loss modeling is still an important issue. Also, the use of the same coefficients for a wide range of frequencies might by questionable.

Another important technique for the future systems is MIMO. Although this is a wide topic, worthy of a book or more by itself, only the theory necessary to do a first and basic MIMO analysis has been treated here. This content will be used in the study of MIMO measurements (which is done in Chapter 6). 



\section{Experimental equipment and data processing}

\section{Introduction}

The equipment necessary to carry out a propagation experiment, either for satellite or wireless communications, is usually expensive. Thus, it is common that Research Groups design and implement their own setups, making a more rational use of their available resources. In this sense, the UPM GTIC-Radiocommunication Research Group has built two satellite beacon receivers: one in the Ka-band $(19.680 \mathrm{GHz})$ and other in the Q-band $(39.402$ $\mathrm{GHz}$ ), allowing the reception of the signals from the KA-SAT and Alphasat satellites, respectively. Also, some ancillary meteorological equipment is co-sited with the satellite receivers for obtaining meteorological parameters such as rain intensity, temperature and humidity. The experimental equipment is located in the rooftop of one of the buildings of the UPM ETSI Telecomunicación.

Advanced equipment is also available for the indoor wireless communication experiments at millimeter-wave frequencies. The core of this setup is from Keysight Technologies, and some other components (e.g. antennas, multipliers and an RF attenuator) were acquired from other manufacturers.

The objective of this chapter is to describe the main technical characteristics of the experimental equipment. First, meteorological instruments used for obtaining ancillary information for the satellite experiments are presented. Later, the main characteristics of the KA-SAT and Alphasat receivers and the associated data processing are described. Finally, 
the setup, the calibration procedure and the signal processing for the millimeter-wave indoor experiments are addressed.

\subsection{Satellite propagation equipment}

\subsubsection{Ancillary meteorological instruments}

Near the satellite beacon receivers, the ancillary meteorological instruments that allow gathering complementary climatological measurements are:

- A meteorological automatic station.

- A rain gauge.

- A laser disdrometer.

- A Micro Rain Radar.

Figure 4.1 shows four devices that have been used to obtain the rainfall rate during the past years.

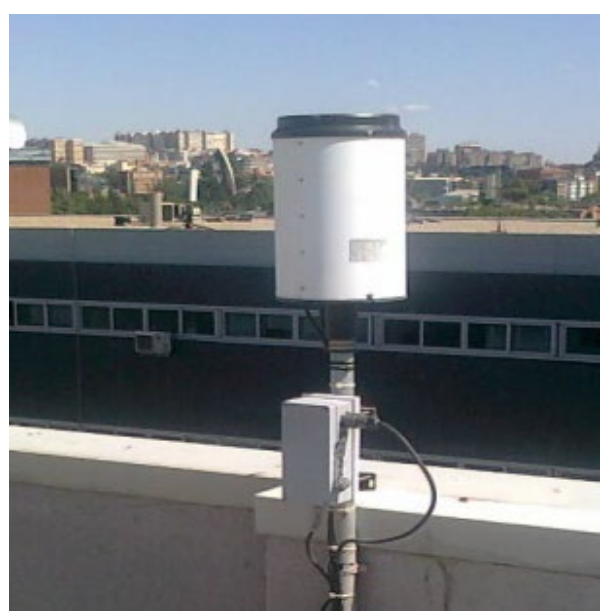

a)

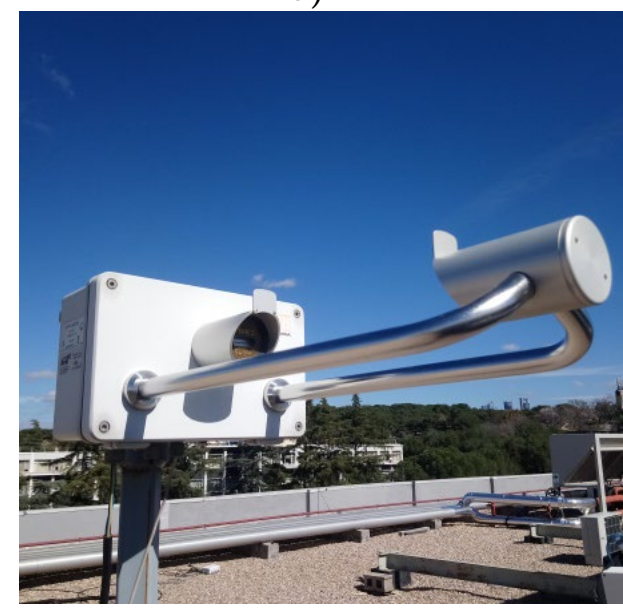

c)

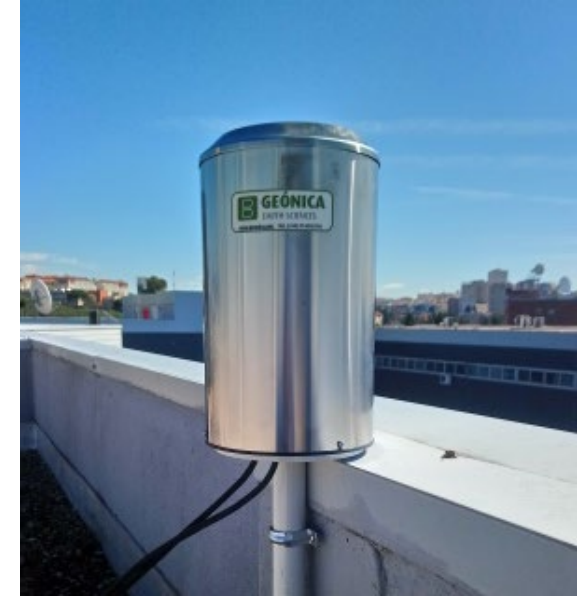

b)

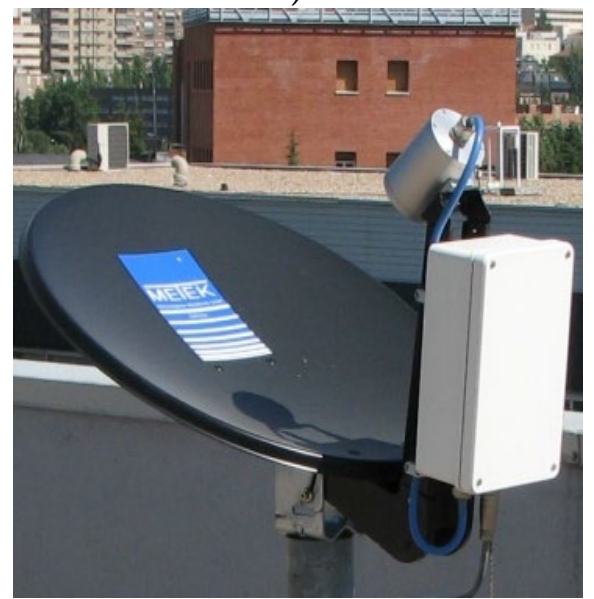

d)

Figure 4.1. Ancillary meteorological instruments. a) Rain gauge (old), b) Rain gauge (new), c) Disdrometer, d) Rain radar. 


\section{The meteorological station}

The station collects the main meteorological data (temperature, relative humidity, wind velocity and direction and atmospheric pressure) by means of their respective measurement instruments and then presents that information every 10 minutes, using specific software.

The Tipping-bucket Rain gauge (old)

A tipping-bucket type rain gauge was used for the first years of measurements. It has two buckets, each one with a water capacity equivalent to $0.1 \mathrm{~mm}$ of rainfall. When it rains, a funnel conduces the water to a bucket which tips-over when it is full, and that instant time is registered in a memory associated to the logger, with an accuracy of seconds. Then, the other bucket is positioned to collect the water, and the cycle is continuously repeated. It can be concluded from the mentioned process that this rain gauge allows to register only the tipover times and not the amount of accumulated rain in a given time period (the total time to fill the bucket can vary from seconds to minutes or even hours), but with this information it is possible to estimate the rainfall rain in 1-minute periods. In November 2017 this device was substituted by a new one with a different operation principle and higher resolution.

The Weighing Rain gauge (new)

The newly installed rain gauge is a DATARAIN-4000 [204] with a collecting area of 200 $\mathrm{cm}^{2}$ developed by Geónica that can measure rain intensities up to $1200 \mathrm{~mm} / \mathrm{h}$ with high accuracy using the weighing principle in the measurement. Like the previous one, the precipitation is gathered using a standard funnel-shaped collecting piece, the open receptacle has also vertical sides designed to prevent rain drops from splashing in or out. The funnel leads the precipitation into a protected rain accumulation chamber where the water is weighed. These measurements are processed by an electronic unit, where an algorithm calculates the precipitation quantities (accumulated rain, intensity of precipitation, etc.) providing rain intensity data with a resolution of $0.02 \mathrm{~mm} / \mathrm{h}$. This unit also manages the low-power operation and the filtering of vibrations generated by strong winds. When the accumulation chamber is full, it is automatically emptied. Besides the difference in the measurement mechanism, another major difference is that the tipping-bucket rain gauge was able to register measurements when a blackout occurs (thanks to its internal batteries) whereas the weighing rain gauge loses these measurements since it needs an external power supply. UPS units were installed to allow the continuous operation of the beacon receivers and the meteorological equipment under short-duration failures of the main power.

\section{The Disdrometer}

An optical disdrometer, known as LPM (Laser Precipitation Monitor), from THIES (http://www.thiesclima.com) is installed near the meteorological station since 2007. It detects 22 diameters and 20 velocity bins of rain drops at surface level, and uses this information to classify the precipitation events (rain, snow, drizzle, hail, etc.). This instrument has a higher resolution and provides more information than the rain gauges, since it allows the accurate registering of the rainfall intensity at 1-minute intervals. 
The disdrometer gives graphical information of rainfall rate during rain events and provides the spectra of the rain drops regarding the size (in $\mathrm{mm}$ ) and fall velocity of the particles (in mm/s), comparing them with the Gunn and Kinzer empirical curve [205].

The rain radar

A 24-GHz vertical-pointing Doppler radar (METEK Micro Rain Radar MRR-2) is used to derive the rain DSD. Specifically, this device provides the drop size and velocity distributions at 30 different heights. The DSD and other estimations derived from this vertically-pointing Doppler radar, are recorded each minute.

A comparison between rainfall rate values obtained with the rain gauge and the disdrometer for a given precipitation event is shown in Figure 4.2. Both instruments follow the rainfall fluctuations adequately. Moreover, the higher resolution of the disdrometer can be verified in the smoothness of the blue line. Small values (close to zero) detected only by the disdrometer appear at the beginning of the figure, while the rain gauge starts to detect values approximately 5 minutes later. However, the main drawback of this type of disdrometer is that it is known that it overestimates the rain rate for high-intensity events.

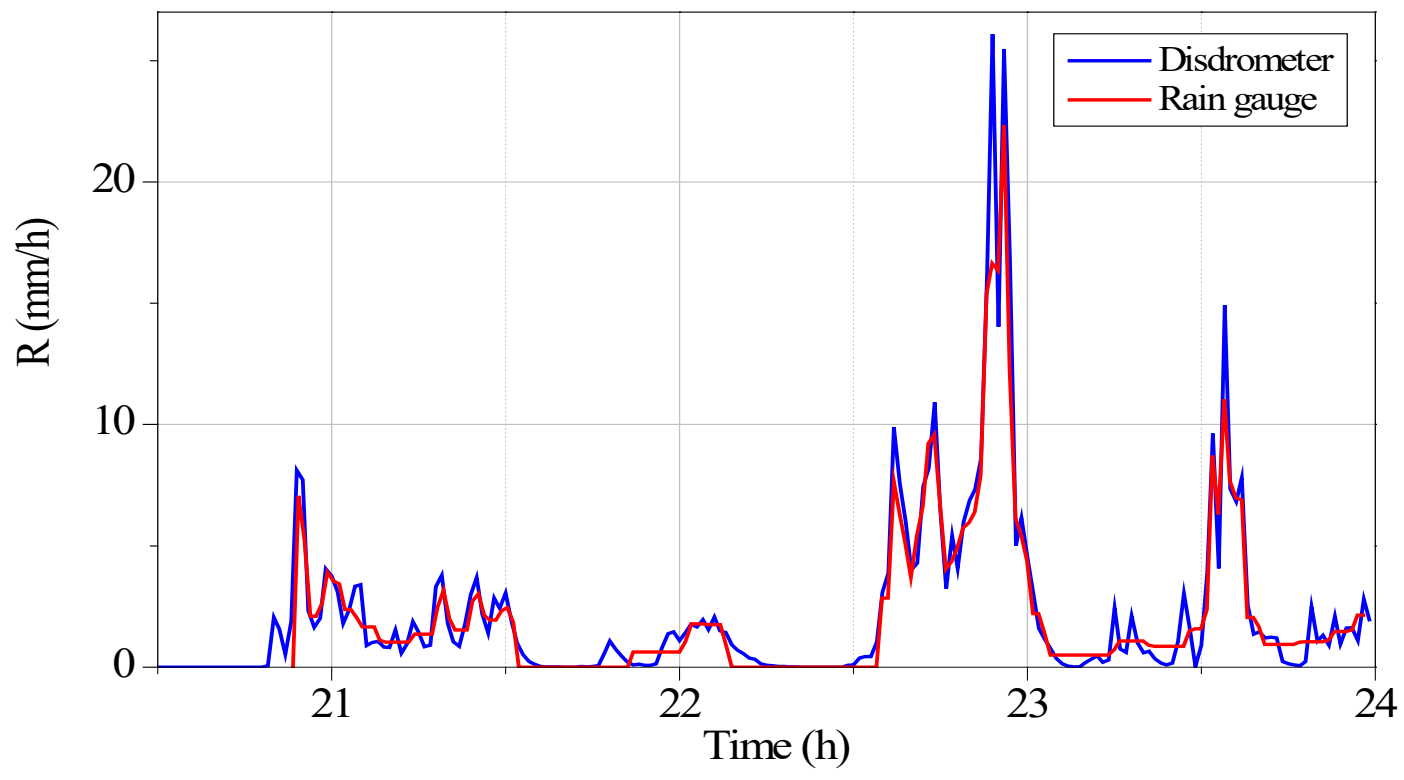

Figure 4.2. Rainfall rate measured with the disdrometer and the rain gauge (old) from 20:30-00:00 of September 21st, 2014.

\subsubsection{Eutelsat KA-SAT satellite propagation receiver}

The Ka-band beacon receiver captures the signal from KA-SAT, a geostationary satellite of the European Telecommunications Satellite Organization (EUTELSAT) launched in December 2010 and located at $9^{\circ}$ East. This satellite allows the provision of wide band services to most Europe and some countries in North-Africa and the Middle-East thanks to its 82 multi-beam system.

The experimental satellite beacon receiver, designed and built at the UPM, was previously used to measure the beacon signal of the Eutelsat Hot Bird (HB) 6 satellite, since July 2006 to June 2013 — the satellite was moved to another orbital position in July 2013. 
The digital receiver was also reused from a previous experiment with the ITALSAT satellite [18] and is based on digital PLL (Phase-Locked Loop) technology. The receiver was successfully re-oriented to KA-SAT since it has a similar frequency, polarization and orbital position as the previously measured satellite. The technical configuration will not be addressed in depth since it can be found in $[\mathbf{1 9}]$ and $[\mathbf{2 0 6}]$. Nevertheless, the main technical and propagation characteristics are provided in Table 4.1.

Table 4.1. Technical and propagation data for KA-SAT experiment in Madrid

\begin{tabular}{|c|c|}
\hline Parameters & Values \\
\hline Satellite name and orbital position & $\mathrm{KA}-\mathrm{SAT}, 9^{\circ} \mathrm{E}$ \\
\hline Frequency and $E I R P$ & $19.680 \mathrm{GHz}$ and $15 \mathrm{dBW}$ \\
\hline Polarization & $\begin{array}{l}\text { Horizontal, } 19^{\circ} \text { from the } \\
\text { horizon }\end{array}$ \\
\hline \multirow[t]{3}{*}{ Site coordinates and altitude } & Latitude $40.45^{\circ} \mathrm{N}$ \\
\hline & Longitude $3.73^{\circ} \mathrm{W}$ \\
\hline & $630 \mathrm{~m} \mathrm{amsl}^{6}$ \\
\hline Azimuth and elevation angles & $160.81^{\circ}$ and $41.41^{\circ}$ \\
\hline Mean distance to the satellite & $37671 \mathrm{~km}$ \\
\hline Antenna type & Centered Cassegrain \\
\hline Gain and Antenna diameter & $\begin{array}{l}\quad 45.7 \mathrm{~dB}, 1.2 \mathrm{~m}(15 \mathrm{~cm} \\
\text { the sub-reflector diameter })\end{array}$ \\
\hline Free space loss $L_{b f}$ & $209.9 \mathrm{~dB}$ \\
\hline Clear sky received power & $-119.3 \mathrm{dBm}\left(L_{g}=0.04 \mathrm{~dB}\right)$ \\
\hline Noise figure $F$ for the receiver noise temperature $T_{r x}=199 \mathrm{~K}$ & $2.3 \mathrm{~dB}$ \\
\hline Noise power density in clear sky $\left(T_{a n t}=30 \mathrm{~K}\right)$ & $-175.0 \mathrm{dBm}$ \\
\hline G/T (Gain/Temperature) in clear sky & $22.1 \mathrm{~dB} / \mathrm{K}$ \\
\hline $\begin{array}{l}\text { Signal to noise spectral density rate } C / N_{0} \text { (Carrier to Noise) in } \\
\text { clear sky }\end{array}$ & $55.7 \mathrm{dBHz}$ \\
\hline Noise power density in fade conditions $\left(T_{a n t}=270 \mathrm{~K}\right)$ & $-171.9 \mathrm{dBm}$ \\
\hline$G / T$ in deep fade condition & $19.0 \mathrm{~dB} / \mathrm{K}$ \\
\hline Sampling frequency of the measurements & $18.66 \mathrm{~Hz}$ \\
\hline Receiver threshold $C / N_{0}$ & $21.5 \mathrm{dBHz}$ \\
\hline Threshold in term of power & $-149.5 \mathrm{dBm}$ \\
\hline Rain margin & $30.2 \mathrm{~dB}$ \\
\hline Outage probability (estimated with ITU-R method [16]) & $<0.001 \%$ \\
\hline
\end{tabular}

The received signal has a sampling frequency of $18.66 \mathrm{~Hz}$. With it, the excess attenuation can be derived, from which rain attenuation and tropospheric scintillation can also be obtained. The signal is amplified and down-converted to an intermediate frequency in the outdoor RF unit that is in the back of the antenna dish of Figure 4.3. From there, it is conducted to the indoor unit, where it is amplified, filtered and again down-converted. Finally, its amplitude is measured by a digital PLL-based receiver. A complete block diagram

\footnotetext{
${ }^{6}$ amsl: above mean sea level.
} 
of the receiver is shown in Figure 4.4. The detailed data processing can be consulted in [19], although it shares several similarities with the processing of Alphasat described in the next sub-section.

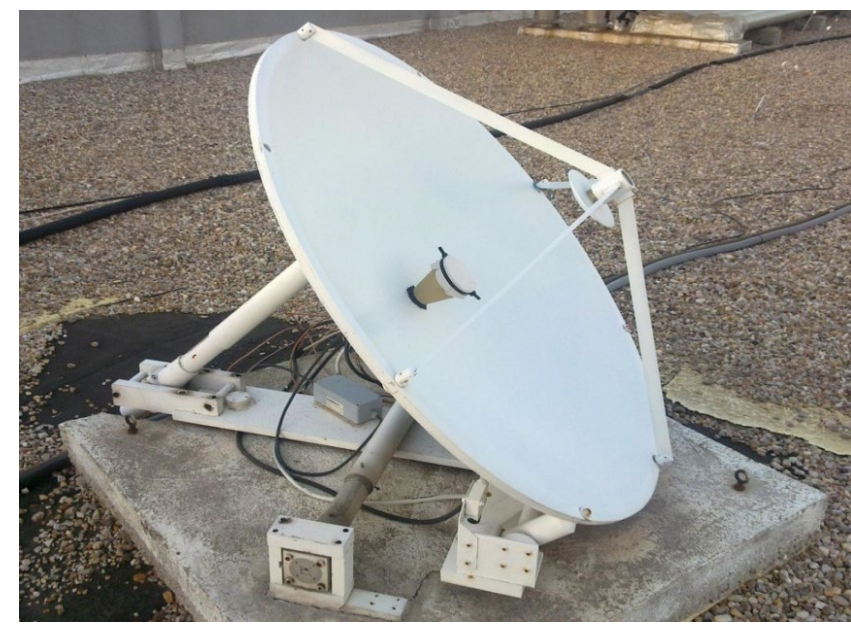

Figure 4.3. Centered Cassegrain receiver antenna used in the KA-SAT experiment.

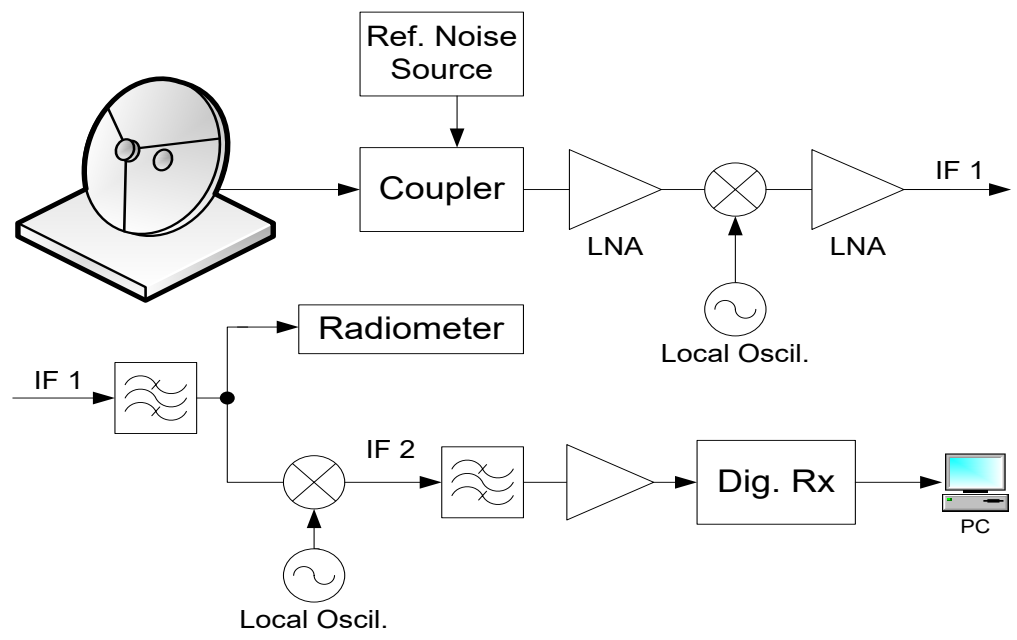

Figure 4.4. Block diagram of KA-SAT satellite beacon receiver [19].

\subsubsection{Alphasat satellite propagation experiment}

The Q-band beacon receiver is oriented to the Alphasat satellite of INMARSAT (the satellite is part of a global fleet for the satellite mobile commercial service in the L-band), which was launched in July 25, 2013, covering most of Europe, North-Africa and some Middle-East areas. It is provided with several TDPs (Technological Demonstration Payloads), and specifically the TDP-5 Aldo Paraboni Q/V Communications and Propagation - developed by Thales Alenia Space and Space Engineering - has as its main objective the assessment of the suitability of the Q/V-band for its future commercial use [207]. Altogether, the objectives of this TDP can be grouped in:

- Communication experiment: Intended to evaluate the performance of links that operate in Q/V bands with interferences and PIMT. 
- Scientific experiment: Oriented to collect additional propagation data from the Ka and $\mathrm{Q} / \mathrm{V}$ bands.

- Technological experiment: Oriented to assess the performance of new hardware in $\mathrm{Q} / \mathrm{V}$ band in the course of the beacon useful life.

The satellite is geosynchronous, but not geostationary: its orbit is not equatorial since it has an inclination, with a maximum value of $3^{\circ}$ above the Equatorial plane. Hence the satellite apparent position is not fixed, rather it is moving along the day describing an ellipse in the sky, with an extension larger than the antenna beam aperture.

Two coherent beacon signals are used in the satellite experiment, one in the Ka-band $(19.701 \mathrm{GHz})$ and the other in the Q-band $(39.402 \mathrm{GHz})$. The satellite coverage areas for Europe are represented in Figure 4.5.

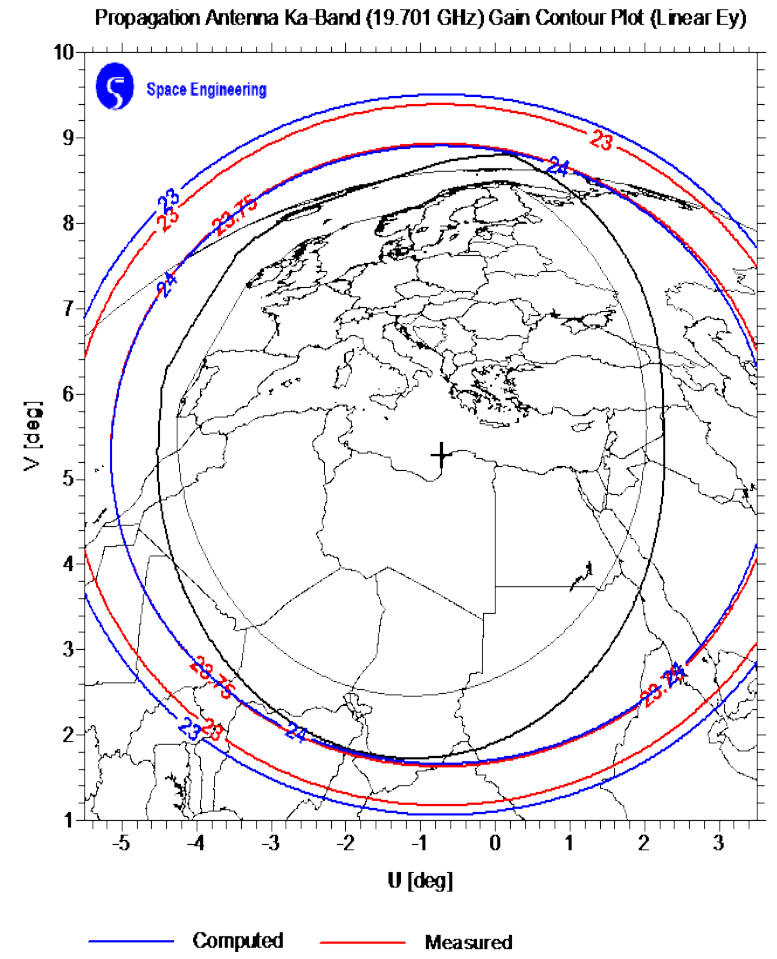

a)

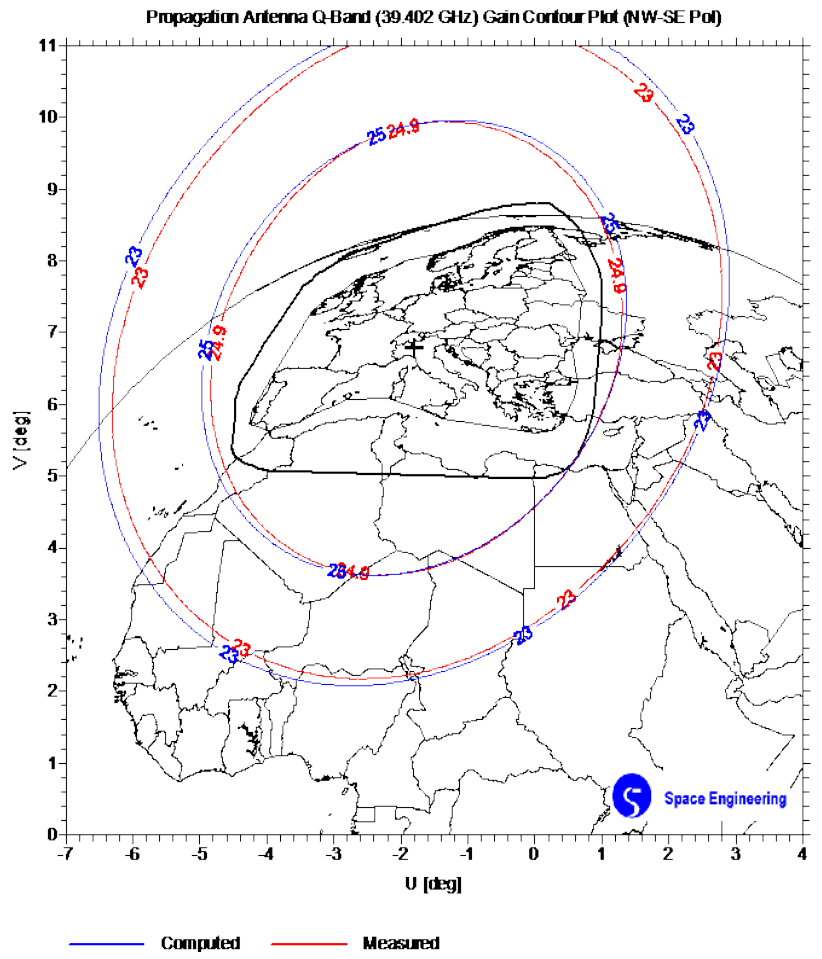

b)

Figure 4.5. TDP5 Aldo Paraboni Scientific experiment, satellite coverage areas for (a) Ka- and (b) Q-band beacons [208].

The GTIC-Radiocommunication Research Group is receiving since March 2014 the 39.402 GHz Alphasat satellite beacon signal. The main characteristics for the Q-band beacon receiver in Madrid are shown in Table 4.2. Due to the inclination in the satellite orbit, the received frequency varies because of the Doppler Effect. The maximum Doppler deviation for Madrid is approximately $2.32 \mathrm{kHz}$.

The receiver block diagram is shown in Figure 4.6, and is composed by two units: the Outdoor Unit (ODU) in which the signal coming from the satellite is received and amplified (besides other secondary processes such as down-conversion) and the Indoor Unit (IDU) in which the main operations are the data filtering, processing and storage. 


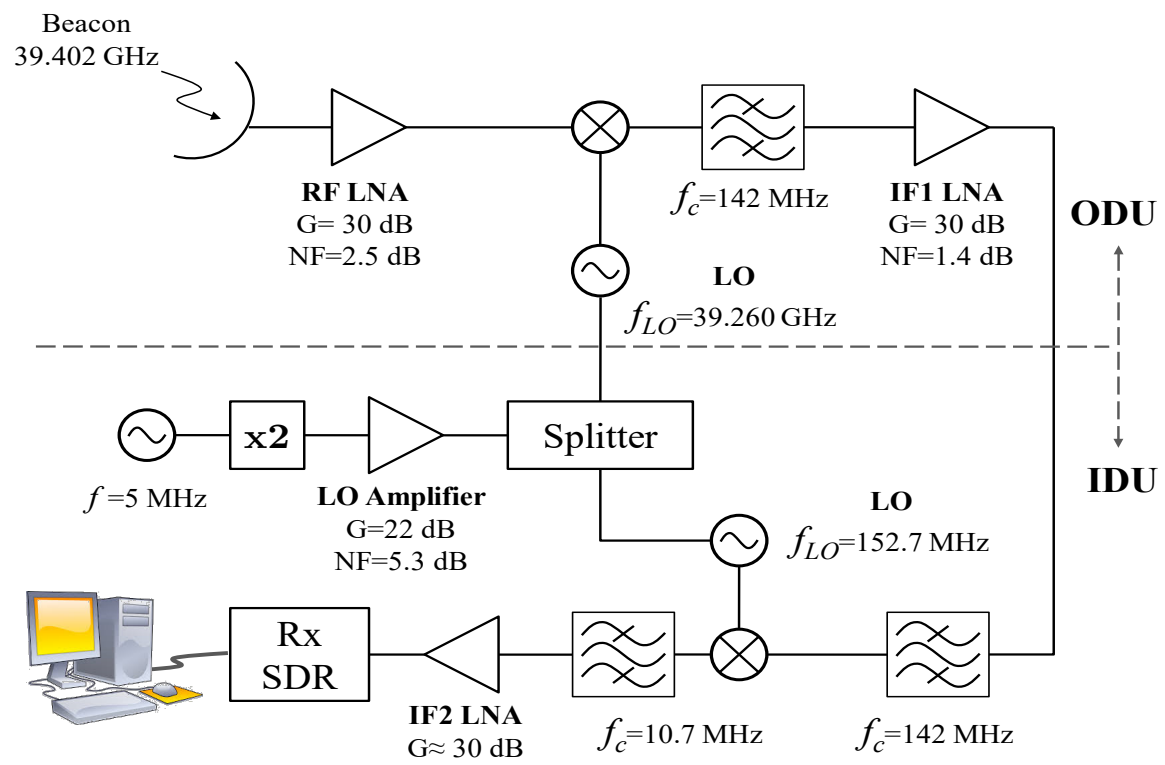

Figure 4.6. Alphasat receiver block diagram.

Table 4.2. Technical and propagation data for the Q-band Alphasat experiment in Madrid

\begin{tabular}{lc}
\hline \hline \multicolumn{1}{c}{ Parameter } & Values \\
\hline Satellite name and orbital position & Alphasat, $25^{\circ} \mathrm{E}$ \\
Frequency and $E I R P($ estimated) & $39.402 \mathrm{GHz}$ and $26.5 \mathrm{dBW}$ \\
Polarization & Linear, $45^{\circ}$ tilted \\
Site coordinates and altitude & Latitude $40.45^{\circ} \mathrm{N}$ \\
& Longitude $3.73^{\circ} \mathrm{W}$ \\
Mean azimuth and elevation angles & $630 \mathrm{~m}$ amsl \\
Mean distance to the satellite & $139.5^{\circ}$ and $34.5^{\circ}$ \\
Antenna type & $38202 \mathrm{~km}$ \\
Antenna gain and diameter & Centered dish \\
Free space losses $L_{b f}$ & $48.3 \mathrm{~dB}$ and $0.935 \mathrm{~m}$ \\
Clear sky received power (with a gas attenuation $\left.L_{g}\right)$ & $216.0 \mathrm{~dB}$ \\
Noise figure $F$ with a receiver noise temperature $T_{r x}=193 \mathrm{~K}$ & $-112.2 \mathrm{dBm}\left(L_{g}=1 \mathrm{~dB}\right)$ \\
Clear sky noise power density $\left(T_{a n t}=30 \mathrm{~K}\right)$ & $2.2 \mathrm{~dB}$ \\
$G / T$ in clear sky & $-175.1 \mathrm{dBm} \mathrm{@} 1 \mathrm{~Hz} \mathrm{BW}$ \\
Signal to noise spectral density rate $C / N_{0}$ in clear sky & $24.8 \mathrm{~dB} / \mathrm{K}$ \\
Noise power density in fade conditions $\left(T_{a n t}=270 \mathrm{~K}\right)$ & $62.9 \mathrm{dBHz}$ \\
G/T in deep fade condition & $-172.0 \mathrm{dBm} \mathrm{@} 1 \mathrm{~Hz} \mathrm{BW}$ \\
Measurement sampling frequency & $21.6 \mathrm{~dB} / \mathrm{K}$ \\
Receiver threshold $C / N_{0}$ & $18.78 \mathrm{~Hz}$ \\
Threshold in terms of power & $21.9 \mathrm{dBHz}(\mathrm{estimated})$ \\
Rain margin & $-150.0 \mathrm{dBm}$ \\
Outage probability (estimated with ITU-R method $[\mathbf{6 m})$ & $37.8 \mathrm{~dB}$ \\
\hline \hline
\end{tabular}




\subsubsection{Outdoor unit (ODU)}

The ODU is located near the antenna inside a case that protects it from the weather conditions, on the rooftop of one of the UPM buildings. In it, the signal pre-processing is carried out by means of amplification and filtering. Moreover, in the ODU a first frequency down-conversion to an intermediate frequency of $142 \mathrm{MHz}$ takes place, later the signal is fed to the cables that connect with the Indoor Unit (IDU).

The receiver antenna is a Q-par center dish, composed by a parabolic reflector QSR900$337 \mathrm{M}$ and a QSF22F feeder with the following characteristics:

Table 4.3. The QSR900-3337M / QSF22F antenna characteristics

\begin{tabular}{lc}
\hline \hline Parameters & Value \\
\hline Frequency range & $26-40 \mathrm{GHz}$ \\
Gain at $39.40 \mathrm{GHz}$ & $48.4 \mathrm{dBi}$ \\
Reflector diameter & $935 \mathrm{~mm}$ \\
3 dB Bandwidth $(39.40 \mathrm{GHz})$ & $0.6^{\circ}$ \\
Focus distance & $337 \mathrm{~mm}$ \\
Front/Back rate & 0.37 \\
Weight & $7 \mathrm{~kg}$ \\
\hline \hline
\end{tabular}

A positioning device and a U-shape piece from Instro (http://www.instro.com) are located in the antenna base (see Figures 4.7 and 4.8), and are used to correct in a mechanical way the antenna steering direction toward the satellite, since the orbit is not perfectly geostationary. The positioning device is controlled by MATLAB-based software that uses either the Two-Line Elements (TLE) data obtained automatically from Celestrak (http://www.celestrak.com) or the Orbit Ephemeris Message (OEM) files provided by the satellite operator to calculate the steering direction.
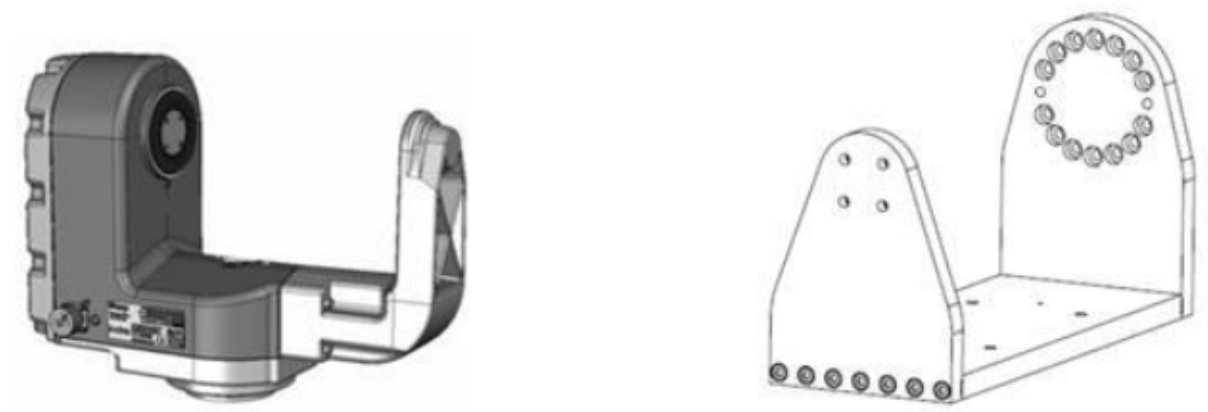

Figure 4.7. Positioning device (left) and U-shape piece (right).

The receiver (see Figure 4.9) has been designed to minimize the noise power and the temperature, and to maintain a good $\mathrm{C} / \mathrm{N}$ ratio. With this aim low noise amplifiers have been used in the RF (radio frequency) and IF (Intermediate frequency) stages. 
The RF oscillator is a phase-locked oscillator (PLO), tuned at a frequency of $39.26 \mathrm{GHz}$. The required reference signal of $5 \mathrm{MHz}$ is delivered by a cable from a signal generator (oscillator) located in the Radiocommunication Laboratory room approximately $40 \mathrm{~m}$ apart.

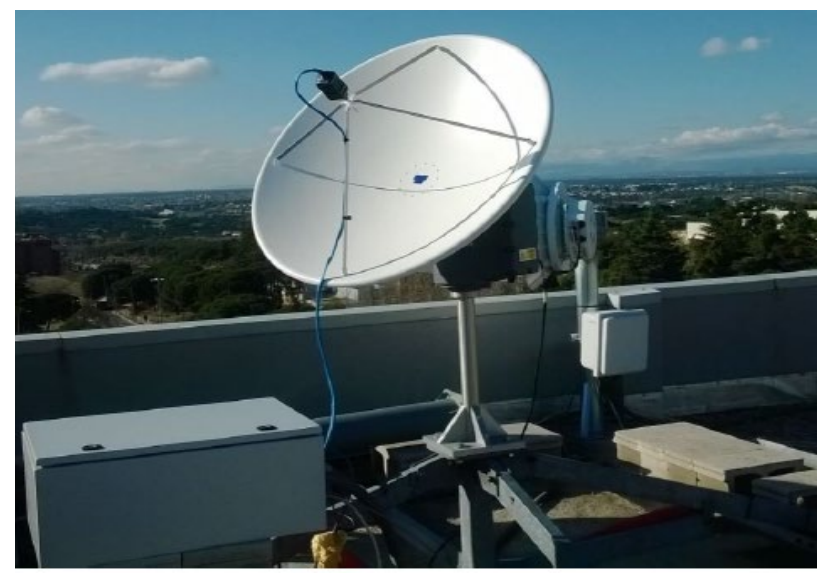

Figure 4.8. Dish antenna at the center and ODU box in the left-bottom part.

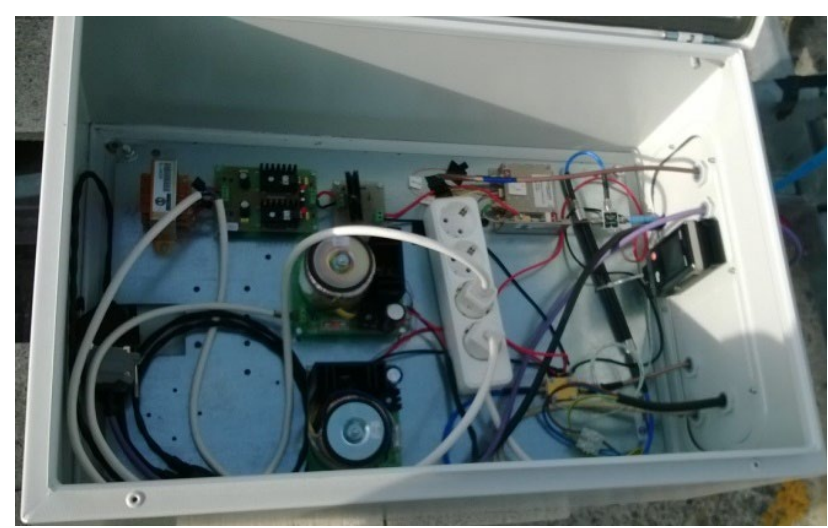

Figure 4.9. RF components inside the ODU.

The frequency down-conversion from $39.402 \mathrm{GHz}$ to the first intermediate frequency of $142 \mathrm{MHz}$ is carried out by a mixer capable to reject the image frequency and in that way maintaining the $\mathrm{C} / \mathrm{N}$ ratio. A filter has been located after the mixer because this is a nonlinear device and can produce spurious signals.

In the final step, an amplifier is used to increase the signal level by $30 \mathrm{~dB}$ and then the signal is guided by cable to the IDU.

\subsubsection{Indoor unit (IDU)}

In the IDU, the signal coming from the ODU is filtered in order to eliminate possible interference captured by the coaxial cable; later it passes through a second mixer that converts it from the previous frequency of $142 \mathrm{MHz}$ to $10.7 \mathrm{MHz}$ using a $152.7 \mathrm{MHz}$ frequency locked oscillator. The output signal from the mixer is later pass-band filtered at 10.7 MHz and amplified, since some losses occur during the process.

The signal digitalization step takes place in the Software Defined Radio (SDR) device, being sampled by an Analog to Digital Converter (ADC) that receives the entire or a part of 
the $0-40 \mathrm{MHz}$ frequency band depending of the pre-selection filter - that can also amplify or attenuate the signal - and samples it at $80 \mathrm{MHz}$. Then, the resulting signal is filtered in the digital domain around $10.7 \mathrm{MHz}$, decimated to a sampling frequency of $48 \mathrm{kHz}$ and bandpass sampled in I-Q domain, resulting in a base-band transformation. The resulting samples are transmitted via USB to a PC to be temporarily stored while they are processed. The involved devices are shown in Figure 4.10.

Later, the signal and the noise levels are calculated in real time from the temporary files stored in the PC using an algorithm based in the Fast Fourier Transform (FFT). The results, that represent the received power and noise level, have a sampling frequency of 18.78 samples/s. Finally, 4 files of 6 hours each for a 24-hours typical day are generated (files with durations different from 6 hours will be created in the case of information loss due to receiver malfunctions or blackouts).

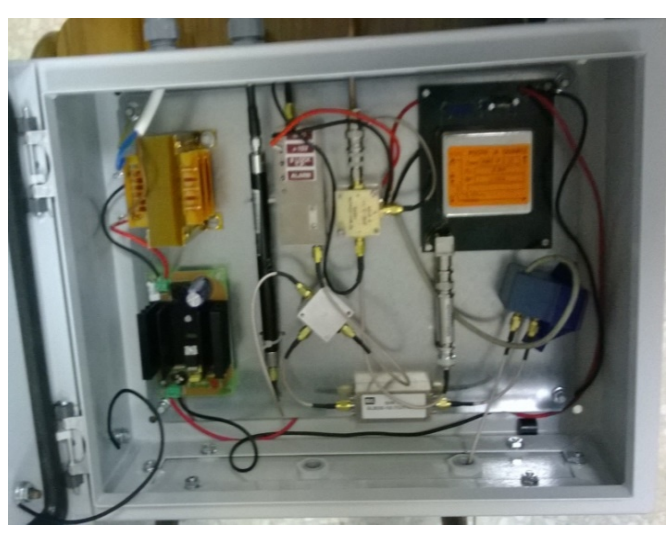

a)

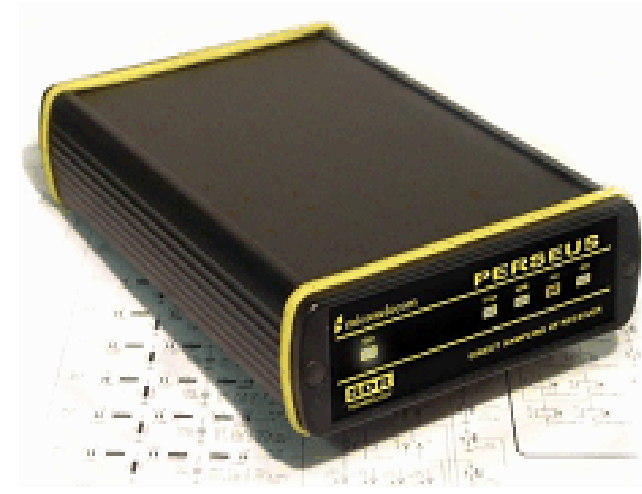

b)

Figure 4.10. IDU elements. a) RF components b) SDR.

\subsubsection{Measurement processing}

The first stage of the beacon signal processing has as objective to generate a normalized file per day and in that way all the resulting daily files will have the same number of samples - this might not occur at the beginning due to occasional sample losses by several reasons such as: electrical failure, wrong data reading, etc. To do so, the receiver daily files generated in the previous step are synchronized and combined with a normalized time base of 1622592 samples/day. Besides, the samples are tagged with a flag value that indicates the sample characteristics as Table 4.4 shows:

Table 4.4. Flag vector values for the samples in the first stage

\begin{tabular}{cl}
\hline \hline Value & \multicolumn{1}{c}{ Description } \\
\hline 0 & Valid data (The most common value in a normal day). \\
1 & Invalid data (Spikes in the received signal due to several reasons). \\
2 & Doubtful data (Obtained when the receiver margin is exceeded). \\
3 & Lack of measurements (Due to a system turn-off). \\
4 & Interpolated data (Samples used to complete the total sample number). \\
5 & Corrected data (Those easily corrected at this stage). \\
\hline \hline
\end{tabular}


With this data a MATLAB .mat type file is created for each day with the power time series, the flag value for each sample and the initial time of the file. The power time series has a systematic component with a slow variation due to different effects: thermal variations of the gain for the different stages, in particular the amplifiers placed in the outdoor unit, imperfect tracking of the orbital satellite movement, and absence of satellite tracking in the first months of the experiment, when the antenna pointing was fixed. All these effects can be eliminated by extracting a reference level.

In the first months of the experiment the orbit inclination was small and the antenna pointing was kept fixed, with the result that amplitude variations of up to a few $\mathrm{dB}$ were produced. The slow system fluctuation (seen as a sinusoidal variation in the signal during the day in Figure 4.11) has been eliminated by means of the reference processing. For the first months of measurements this variation was caused mostly by using a fixed pointing. The variation dropped from approximately $5 \mathrm{~dB}$ to $1-1.5 \mathrm{~dB}$ peak to peak (in clear sky conditions) when an automatic pointing system was set into operation in September 2014. These diurnal fluctuations of the amplitude seem to be well correlated with temperature changes, so apparently antenna pointing defects have a negligible influence from that date on.

The excess and total attenuation can be estimated from the daily files. The raw power measurements $P_{r}$ obtained from the beacon receiver can be calculated as:

$$
P_{r}(d B)=E I R P-L_{b f}+G_{a n t}+G_{r x}-A_{g}-A_{c}-A_{r}-S_{c}
$$

With:

$$
\begin{aligned}
A_{g} & : \text { Gas attenuation. } \\
A_{c} & : \text { Cloud attenuation. } \\
A_{r} & : \text { Rain attenuation. } \\
S_{c} & : \text { Scintillation contribution. }
\end{aligned}
$$

The first four terms of (4.1) are the satellite EIRP, free-space losses $\left(L_{b f}\right)$, antenna gain $\left(G_{a n t}\right)$ and receiver gain $\left(G_{r x}\right)$, and can vary in time, i.e. the EIRP varies due to thermal fluctuations within the satellite during the day, the EIRP and the $L_{b f}$ can fluctuate because of the orbit inclination, $G_{a n t}$ is affected by the antenna miss-pointing, $G_{r x}$ varies mostly by thermal effects in the ODU [209].

The reference level that would be received in clear sky condition (and having into consideration that there are not fast variations in the power level, such that produced by satellite maneuvers) is:

$$
P_{r e f}(d B)=E I R P-L_{b f}+G_{a n t}+G_{r x}-A_{g}
$$

Then:

$$
P_{r}(d B)=P_{r e f}-A_{c}-A_{r}-S_{c}
$$


In order to cancel out the scintillation contribution, a low pass filtering can be applied. In the absence of cloud and/or rain events, the reference is calculated as the average of the received signal in such conditions $\left\langle P_{r}\right\rangle$ :

$$
P_{r e f}(d B)=\left\langle P_{r}\right\rangle
$$

Excess attenuation is calculated by taking as reference the clear sky condition, which means that it includes attenuation due to rain and clouds, as well as amplitude fluctuations due to scintillation. To extract the reference signal to be used in the excess attenuation estimation, an experienced operator uses the ancillary information given by the co-sited instruments to identify the different events of rain and clouds in a certain interval. Within the events, the reference level is estimated from the averaged signal level received a time before and after the event. Intermediate points with 0-dB excess attenuation are also used in some cases. In the top of Figure 4.11, the following are shown: the power signal (in blue color), the reference level (represented with a dotted black line) and the points selected by the operator to draw it when an event of rain (in the site or along the path) and/or cloud occurs (red asterisk). In the bottom of Figure 4.11 the rainfall rate information derived from the rain gauge (pluviometer) and disdrometer is displayed. A very good correlation is observed in this case between the signal fades and the rainfall intensity. Out of the events, the reference level is obtained by using a slicing low-pass filter.

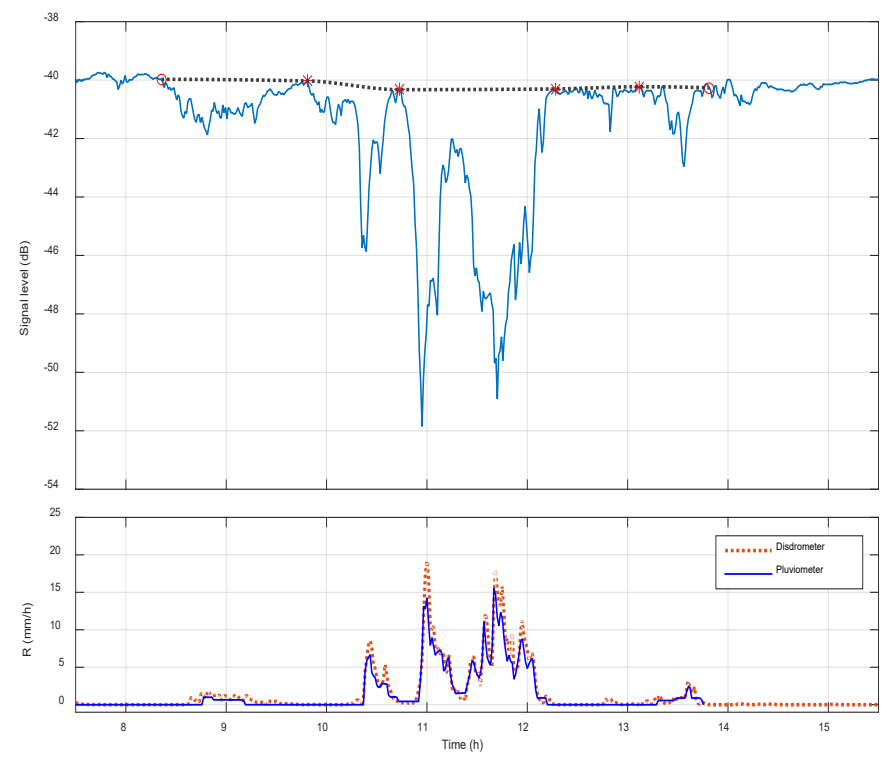

Figure 4.11. Excess attenuation referencing procedure (November 11, 2014).

It is worth to mention that the signal level in Figure 4.11 has been low-pass filtered to improve the visualization of cloud and rain attenuation events. A realistic visualization of the excess attenuation reference signal time series (Exc. Att. Reference) for an entire day (June 15, 2015) is seen in Figure 4.12. Then, the excess attenuation time series are obtained as:

$$
A_{\text {exc }}(d B)=P_{\text {ref }}-P_{r}
$$

For that specific day, the excess attenuation time series is shown in Figure 4.13. 


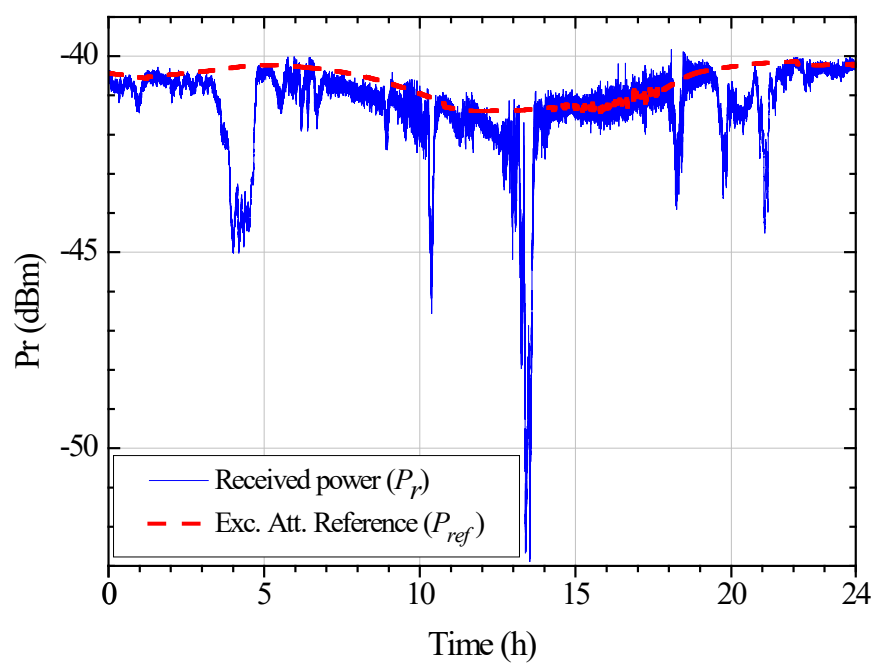

Figure 4.12. Received power and excess attenuation reference signals (June 15, 2015).

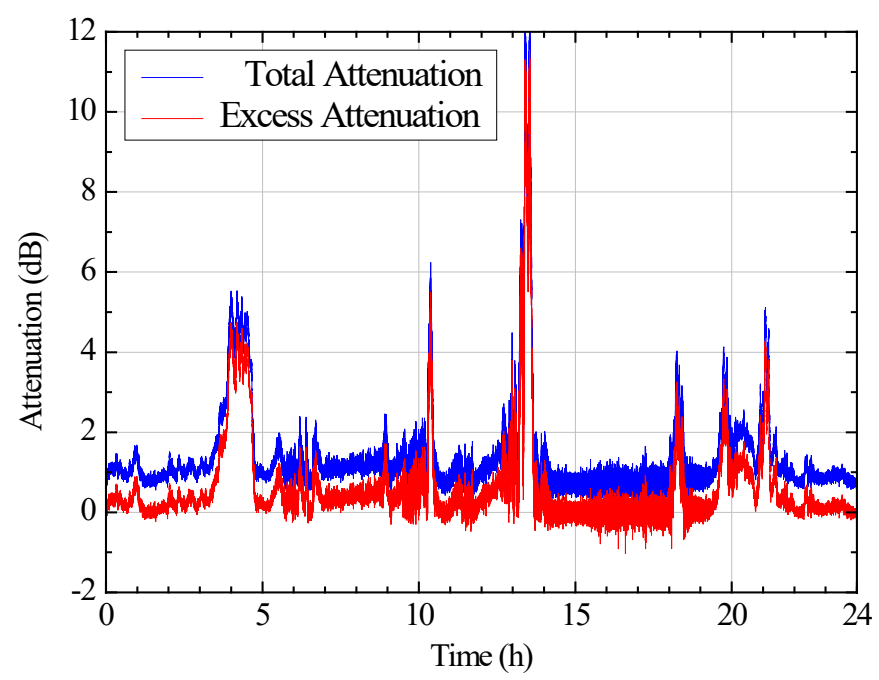

Figure 4.13. Total and excess attenuation time series

(June 15, 2015).

Total attenuation uses as reference the free-space condition (without any atmosphere contribution). This means that it includes the attenuation contributions of gases, rain and clouds as well as scintillation. Then, it is calculated as:

$$
A_{t o t}(d B)=A_{e x c}+A_{g}
$$

The comparison between the total and excess attenuation for the specific day is displayed in Figure 4.13 as example. The difference between total and excess attenuation is the gas attenuation.

For estimating gas attenuation, a co-sited radiometer is desirable (this is the best but also the most expensive option); however, this equipment is not available for this experiment, or the majority of satellite experiments. However, other procedures can be used for estimating the gas attenuation. 
Here, a procedure developed in the GTIC-Radiocommunication Research Group [210] is used to derive the gas attenuation. It uses Zenith Tropospheric Delay (ZTD) information of the IGS (International GNSS Service) data from the "Villafranca del Castillo" ESA (European Space Agency) station (19 km from the site and at a similar height). This procedure has been used to validate radiometric measurements of atmospheric attenuation at 19.7 GHz with very good results.

\subsection{Millimeter-wave indoor equipment}

For assessing the indoor path loss propagation and gathering MIMO measurements, a transmitter and receiver setup has been configured using different subsystems and RF components and their connection elements. Most of the sub-systems have been purchased from the manufacturer Keysight Technologies. A block diagram of the transmitter-receiver scheme with its main characteristics and components is represented in Figure 4.14. The antenna configuration displayed here may be changed depending on the different experiments.

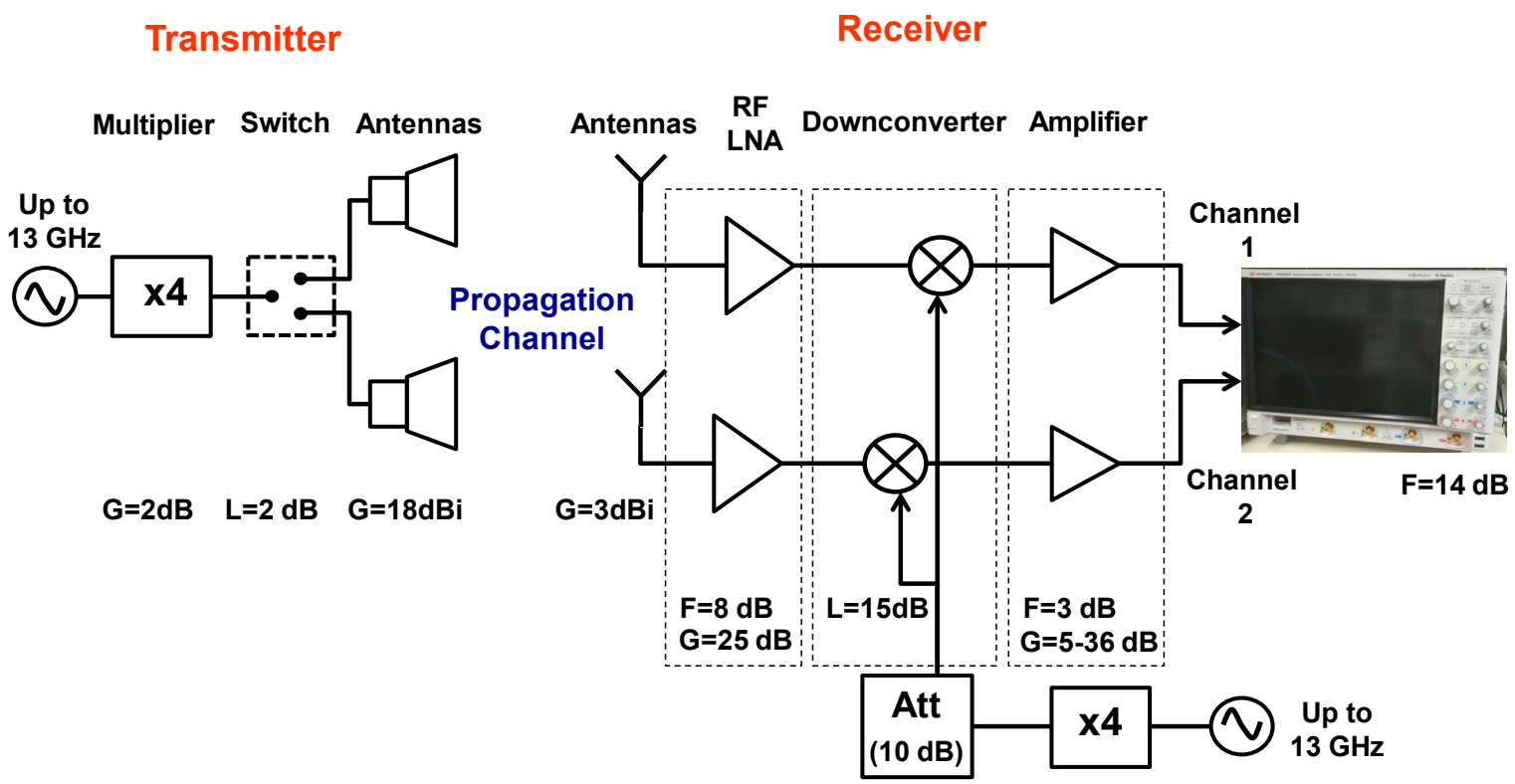

Figure 4.14. Block diagram of the millimeter-wave transmitter-receiver scheme.

\subsubsection{Transmitter unit}

The transmitter unit is composed by an N5173B ENG-X-513 signal generator [211] from Keysight Technologies that can sweep in frequency from $9 \mathrm{kHz}$ up to $13 \mathrm{GHz}$, has a resolution of $0.001 \mathrm{~Hz}$ and can be adjusted in phase in steps of $0.1^{\circ}$. This device has an output power from -20 to $18 \mathrm{dBm}$, with a phase noise at $1 \mathrm{GHz}$ (with an offset of $20 \mathrm{kHz}$ ) of $-122 \mathrm{dBc} / \mathrm{Hz}$.

A fourth-order multiplier MAX4M260400 [212] from Miteq (https://nardamiteq.com/) is placed at the output of the RF signal generator for reaching the bands of interest. The 
multiplier must be excited with an input power from 10 to $13 \mathrm{dBm}$ at a frequency that ranges from 6.5 to $10 \mathrm{GHz}$, hence a maximum limit of $40 \mathrm{GHz}$ is imposed in order to guarantee the technical specifications. This device has a $2 \mathrm{~dB}$ gain and delivers an output power of approximately $12 \mathrm{dBm}$ for the minimum input power.

In order to obtain two channels, a switching device is located after the multiplier and before the antennas (which are located at approximately $1.5 \mathrm{~m}$ height in the transmitter and receiver units); with it, the signal can be delivered to one or the other antenna. The aim of this device is to obtain MIMO measurements.

Two types of antennas are available for this experiment: omnidirectional and horn. The four available omnidirectional antennas are QOM-SL-26-40-K-SG-R type [213] from Steatite Q-par Antennas. Each one provides a gain between 2 and $4 \mathrm{dBi}$ in the 26 to $40 \mathrm{GHz}$ frequency range. The two pyramidal horn antennas are QSH-SL-26-40-K-20 [214] from Steatite Q-par Antennas (https://www.steatite.co.uk/), and their best properties are also specified in the frequency range of 26 to $40 \mathrm{GHz}$, having a gain between 17.7 and $20.9 \mathrm{dBi}$. The use of one type of antenna or the other (or a combination of both) is conditioned by the type of measurements that are taken, for instance: a transmitter with high antenna gain is more suitable to measure the propagation path loss, since longer distances can be analyzed, whereas omnidirectional antennas are more adequate to MIMO measurements.

The transmit signal is an RF tone at the multiplied frequency.

\subsubsection{Receiver unit}

Due to the fact that the propagation loss is severe in millimeter-wave bands, the receiver must be able to receive signals with a very low amplitude level. The receiver unit is more complex than the transmitter one, a reason to move the transmitter while maintaining the receiver in the same position during each set of measurements. The antennas that can be used for the signal reception are of the same type as the transmitter antennas. At least two antennas (omnidirectional or horn) are needed in the receiver to carry out MIMO analyses.

After each of the two receiving antennas, a USB U7227F-8F [215] an RF amplifier is used to increase the signal levels. These amplified signals are guided to an M9362AD01-F50 quad down-converter [216] located in an 18-slots PXI (PCI eXtensions for Instrumentation) M9018A [217] module controlled by a PC. In the down-converter, the signals are translated to a lower frequency (a value of $11.4 \mathrm{MHz}$ was selected) by multiplying them with an LO (local oscillator) signal that comes from a signal generator equal to the one used in the transmitter unit. The LO signal is the result of a frequency-multiplication process similar to the one done in the transmitter, but now the resulting signal is attenuated with a $10-\mathrm{dB}$ SA40-15 attenuator of Fairview Microwave [218], due to the limitation to a maximum of 4 $\mathrm{dBm}$ in the down-converter input power. The resulting signals from the down-conversion process are later amplified by a variable gain IF M9352A-H02 amplifier/attenuator [219] also located in the PXI. These down-converted and amplified signals are captured by a DSOS104A four-channel oscilloscope [220] and stored for later processing. 
Both the transmitter and receiver units are displayed in Figure 4.15. The corridor where the measurement campaign was carried out is seen in the background of Figure 4.15b.

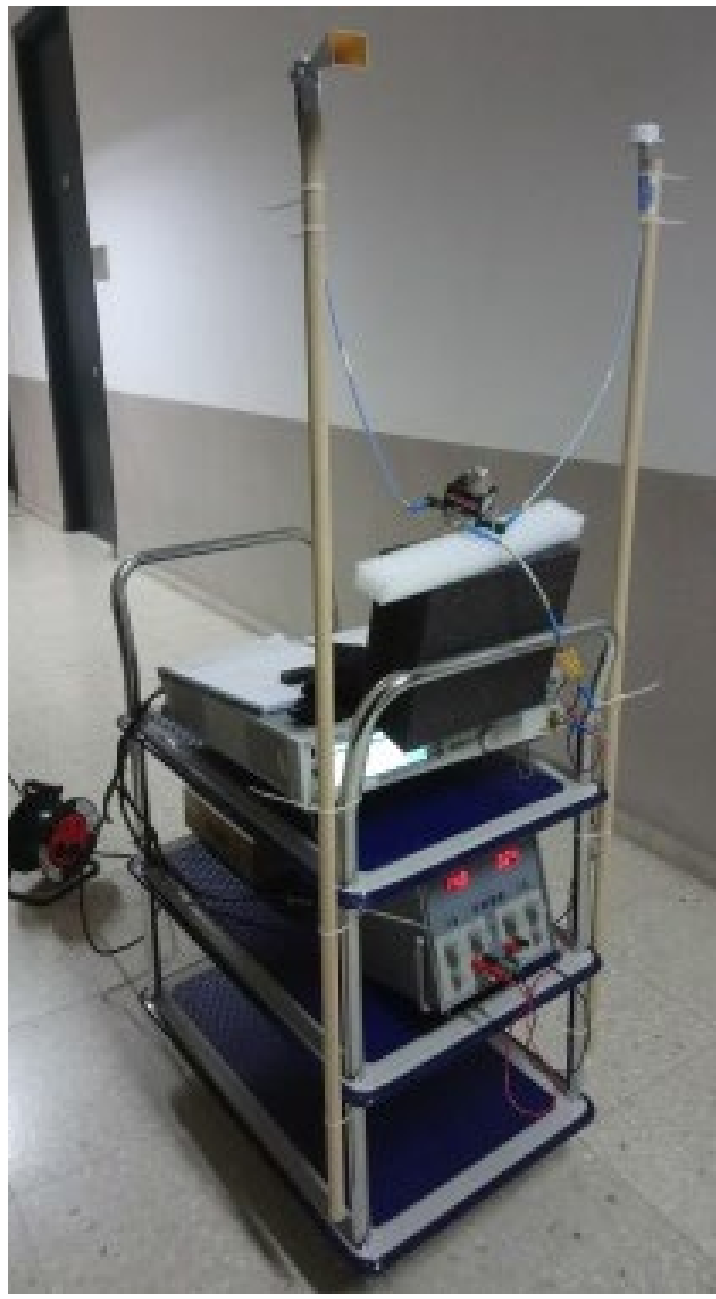

a)

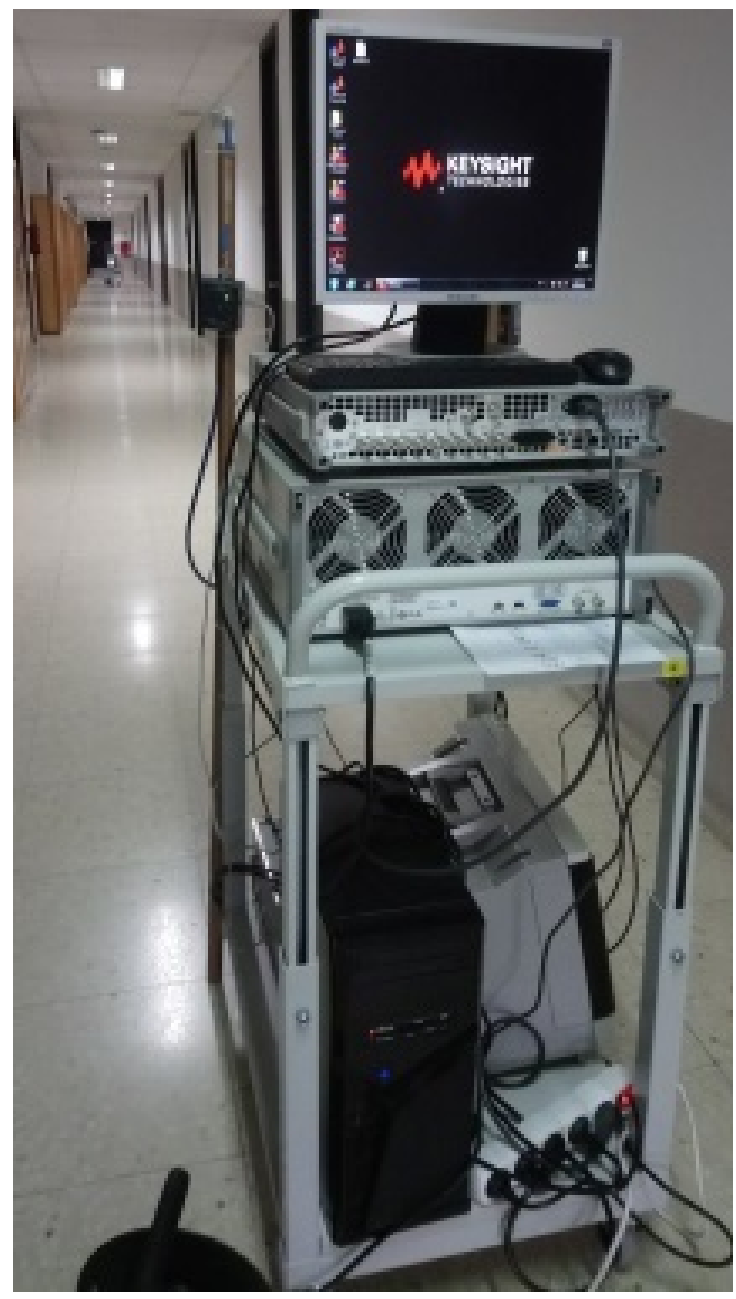

b)

Figure 4.15. Millimeter-wave units, a) transmitter and b) receiver.

The main characteristics of these devices are given in Table 4.5, and the different components of the setup are displayed in Figure 4.16. 
Table 4.5. Millimeter-wave equipment specifications

\begin{tabular}{|c|c|c|}
\hline Devices & Parameters & Values \\
\hline \multicolumn{3}{|c|}{ N5173B ENG-X-513 signal generator } \\
\hline & Frequency range & $9 \mathrm{kHz}$ to $13 \mathrm{GHz}$ \\
\hline & Output power & -20 to $18 \mathrm{dBm}$ \\
\hline & Phase noise @ 1GHz (20 kHz offset) & $-122 \mathrm{dBc} / \mathrm{Hz}$ \\
\hline \multicolumn{3}{|c|}{ USB U7227F-8F RF amplifier } \\
\hline & Input return loss $(2-40 \mathrm{GHz})$ & $8 \mathrm{~dB}$ \\
\hline & Output return loss $(4-40 \mathrm{GHz})$ & $11 \mathrm{~dB}$ \\
\hline & Noise figure $(4-40 \mathrm{GHz})$ & $8 \mathrm{~dB}$ \\
\hline & Maximum input power & $10 \mathrm{dBm}$ \\
\hline \multicolumn{3}{|c|}{ MAX4M260400 multiplier } \\
\hline & Input Frequency range & 6.5 to $10 \mathrm{GHz}$ \\
\hline & Output frequency range & 26 to $40 \mathrm{GHz}$ \\
\hline & Multiplying factor & 4 \\
\hline & Gain & $2 \mathrm{~dB}$ \\
\hline & Input power & 10 to $13 \mathrm{dBm}$ \\
\hline \multicolumn{3}{|l|}{ Switch } \\
\hline & Loss & $2 \mathrm{~dB}$ \\
\hline \multicolumn{3}{|l|}{ SA 40-15 Attenuator } \\
\hline & Power (Average) & $0.5 \mathrm{~W}$ \\
\hline & Power(Peak) & $100 \mathrm{~W}$ \\
\hline & Frequency range & $0-40 \mathrm{GHz}$ \\
\hline & Attenuation & $10 \mathrm{~dB}$ \\
\hline \multicolumn{3}{|c|}{ M9362AD01-F50 down-converter } \\
\hline & $\mathrm{RF}$ frequency range & $0.01-50 \mathrm{GHz}$ \\
\hline & LO frequency range & $0.01-26.5 \mathrm{GHz}$ \\
\hline & FI frequency range & $0.01-50 \mathrm{GHz}$ \\
\hline & Maximum RF input power & $10 \mathrm{dBm}$ \\
\hline & Maximum LO input power & $4 \mathrm{dBm}$ \\
\hline & Loss & 10 to $25 \mathrm{~dB}$ \\
\hline & Channel bandwidth & $1.5 \mathrm{GHz}$ \\
\hline & Number of channels & 4 \\
\hline \multicolumn{3}{|l|}{ IF M9352A-H02 } \\
\hline \multirow[t]{5}{*}{ Amplifier/Attenuator } & Frequency range & 1-2000 MHz \\
\hline & Noise figure & $3 \mathrm{~dB}$ \\
\hline & Maximum gain & $36 \mathrm{~dB}$ \\
\hline & Minimum gain & $5 \mathrm{~dB}$ \\
\hline & Number of channels & 4 \\
\hline \multicolumn{3}{|l|}{ DSOS104A Oscilloscope } \\
\hline & Maxi mum sampling rate & $20 \mathrm{GSa} / \mathrm{s}$ (2 channels) \\
\hline & $\mathrm{ADC}$ & 10 bits \\
\hline & Noise figure & $14 \mathrm{~dB}$ \\
\hline
\end{tabular}




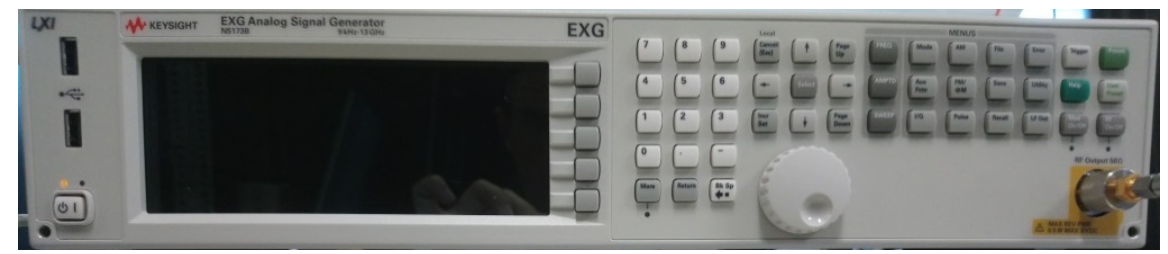

a)

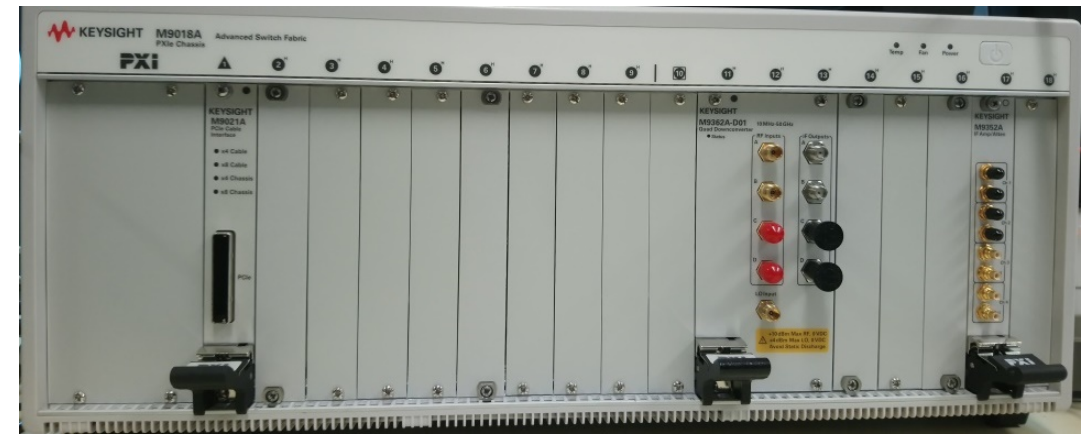

c)

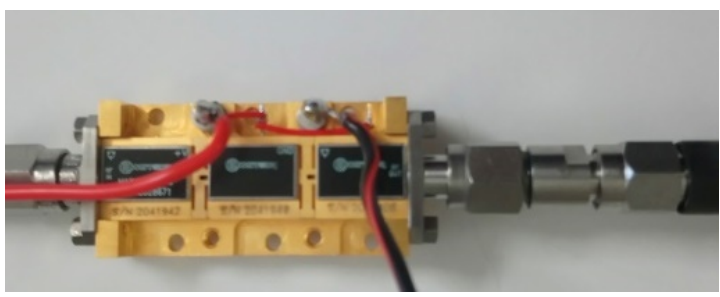

e)

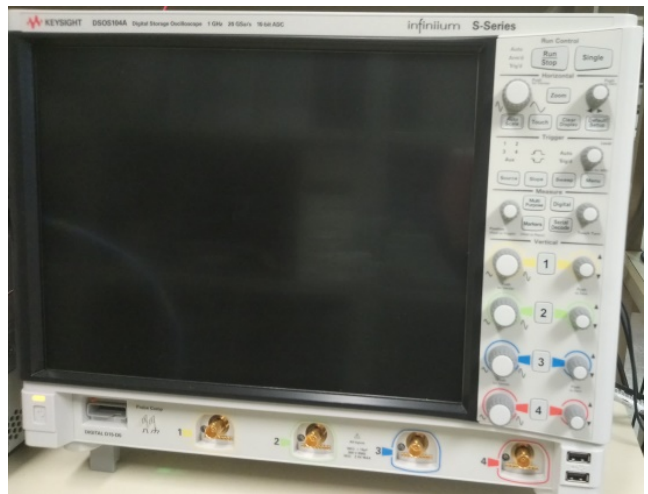

g)

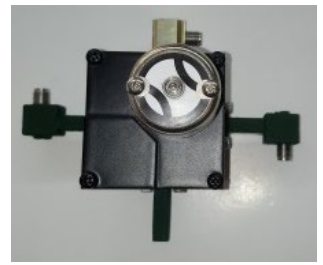

b)

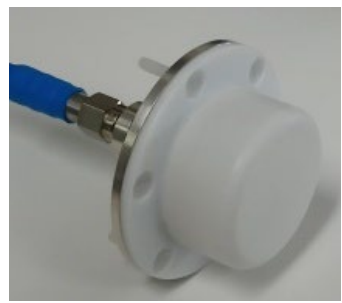

d)

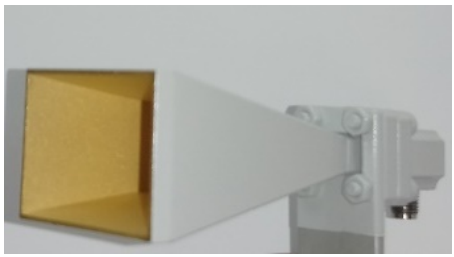

f)

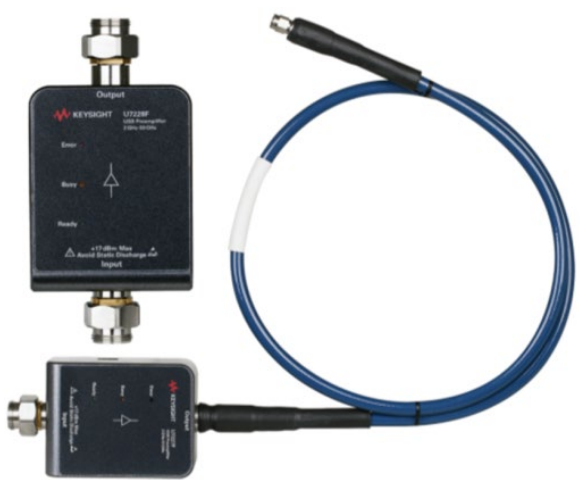

h)

Figure 4.16. Millimeter-wave setup: a) Signal generator, b) switching device with a $50 \Omega$ load in the bottom port, c) PXI, with the controller in slot 1, down-converter in slots 11 to 13 and IF amplifier/attenuator in slot 17, d) omnidirectional antenna, e) multiplier with the attenuator in the right part, f) pyramidal horn antenna, g) oscilloscope and $h$ ) RF amplifier.

\subsubsection{Data Processing}

Once the measurements are collected, a post-processing stage is carried out in order to change the data format into another one simpler to use and to obtain the relevant information about the propagation channel. In this Thesis two kinds of measurements were taken: one for obtaining the received power (and from there the path loss can be derived, 
either for LOS or NLOS conditions) and the second oriented to estimate the $2 \times 2$ MIMO channel matrix (for which the switching device must be used).

First, the signals obtained at the oscilloscope are saved into files with HDF5 format to be processed with a MATLAB program. The signal duration is approximately $3 \mathrm{~s}$. The processing essentially down-samples and filters the received signal in order to increase the SNR. More specifically, each signal (which has been down-converted to $11.4 \mathrm{MHz}$ ) is captured by the oscilloscope at 40 Msamples/s. Later, the sampled signals are downconverted by the program to a $10-\mathrm{kHz}$ carrier and down-sampled to $40 \mathrm{ksamples} / \mathrm{s}$.

The next step is applying the filter, whose purpose is to reduce noise and unwanted signals. Since the signal is essentially a $3 \mathrm{~s}$ portion of a sinusoid, the filter bandwidth can be very narrow. But the impulse response of the filter should be shorter than the signal duration, otherwise the filter output will not reach its steady regime and signal energy will be lost. In other words, the filter bandwidth should be slightly larger than the inverse of the signal duration. An 8th-order band-pass Butterworth filter with $3-\mathrm{Hz}$ bandwidth has been selected. After this filtering process, the energy of the signal is computed.

With the described setup, frequency errors of the order of a few hundreds of $\mathrm{Hz}$ have been observed in the received signal (this represents approximately $0.01 \mathrm{ppm}$ for the transmitted carriers). Therefore, in order to identify the carrier frequency, the $3-\mathrm{Hz}$ filter is centered at different frequencies within a $\pm 1-\mathrm{kHz}$ range around the nominal frequency of 10 $\mathrm{kHz}$, moving it every $3 \cdot(1 / 4) \mathrm{Hz}$ in the $2 \mathrm{kHz}$ range. The output with the maximum power is taken as the actual received signal. This procedure is done for the eight frequencies around the central frequencies either for the 26 or $39.17 \mathrm{GHz}$ measurements at $20 \mathrm{MHz}$ steps (four below and above) and three times in each of the frequencies, obtaining a bandwidth of 140 $\mathrm{MHz}$. Finally, a single value with the average power of these 24 measurements is stored for each distance in each case.

\subsubsection{Calibration procedure}

The goal of the calibration procedure is to estimate a calibration constant $(K)$ encompassing all system contributions to the received power (i.e. antenna gains, device losses/gains, cable losses, etc.) other than the propagation path loss $(P L)$.

The receiver power varies from one distance to another, fluctuating around a mean value. The calibration constant was estimated at distances in the $3-15 \mathrm{~m}$ range without nearby obstacles using the horn antennas at both ends. In these conditions, propagation could be considered to be essentially in free space (FS), having $P L(d)=F S P L(d)=20 \log (4 \pi d / \lambda)$, with distance $d$ and wavelength $\lambda$, and neglecting the reflection and diffraction contributions. Then, $K$ can be obtained from (4.12) as the sum of the mean received power for the previous conditions $\left\langle P_{r}\right\rangle$ and the FSPL:

$$
K(\mathrm{~dB})=\left\langle P_{r}\right\rangle(\mathrm{dBm})+F S P L(d)(\mathrm{dB})
$$

With this value, the path loss can be calculated with (4.13) for the case of using a horn antenna in each end (horn-horn configuration): 


$$
P L_{\text {horn }}(d)(\mathrm{dB})=K(\mathrm{~dB})-P_{r}(\mathrm{dBm})
$$

If a receive omnidirectional antenna is used instead, the difference between the gains of the horn $\left(G_{\text {horn }}\right)$ and the omnidirectional $\left(G_{o m n i}\right)$ antennas must be taken into account (hornomni configuration):

$$
P L_{\text {omni }}(d)(\mathrm{dB})=K(\mathrm{dBm})-P_{r}(\mathrm{dBm})-\left(G_{\text {horn }}-G_{\text {omni }}\right)(\mathrm{dBi})
$$

When more than one channel is used they must be separately calibrated, since they are not identical (some differences may arise from the attenuation of cables and/or antenna gains).

\subsection{5. $\quad$ MIMO processing}

The MIMO configuration is a $2 \times 2$ omnidirectional antennas setup (two antennas at each end). Each measurement has a duration of approximately $3 \mathrm{~s}$. The transmitted sine signal departs from only one antenna at a time and is received by two antennas, amplified, downconverted and finally recorded by two oscilloscope channels. The transmission is switched between the two transmit antennas in the course of the measurement. To be rigorous with the notation, these MIMO measurements can be classified as SIMO, Single Input Multiple Output, at each instant, but since each measurement is taken in a short lapse of time, the stability of the channel seen by each transmit antenna is guaranteed, then, their combination can be considered as MIMO.

The measurements were taken at four frequencies spanned $400 \mathrm{MHz}$ in the $39 \mathrm{GHz}$ band for several LOS and NLOS positions. The processing of the received signal at this stage is similar to the one done for path loss. The signals are down-converted to $11.4 \mathrm{MHz}$, regardless of the millimeter-wave frequency from which they were taken. The objective of using a range of frequencies is to obtain a higher variability in the measurements than if all of them were gathered at the same frequency (in that way the channel richness could be assessed). Also, a second down-conversion to $10.15 \mathrm{kHz}$ is applied. After that, the signals are band-pass filtered with a third-order Butterworth filter with bandwidth around $2 \%$ of the central frequency.

Figure 4.17 shows a measurement interval and is used for a better explanation. In the 3 -s lapse, the change between the transmit antennas is carried out (it can be identified by the abrupt decrease in the signal level for both channels) using the switching device. This takes a time small enough (up to $250 \mathrm{~ms}$, otherwise the measurement is discarded) to consider that both antennas are transmitting through the same channel even if they are transmitting at different times. The switching often occurs near the middle of the measurement time and is done manually by an experienced operator (due to this and the mechanical nature of the device longer times may occur, which are discarded as it was previously mentioned). Before the switching, the Transmit Antenna 1 is transmitting and the signal is being received by receiver 1 and 2 ( $R x 1$ and $R x 2$ in Figure 4.17). After the switching, Transmit Antenna 1 is off and now the signal is being transmitted by Transmit Antenna 2. 


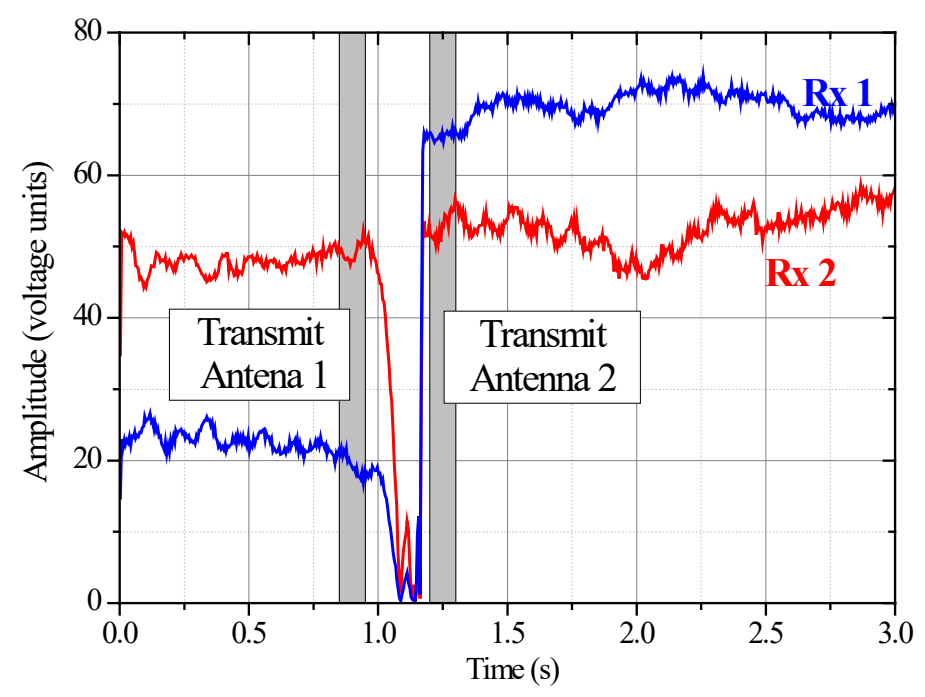

a)

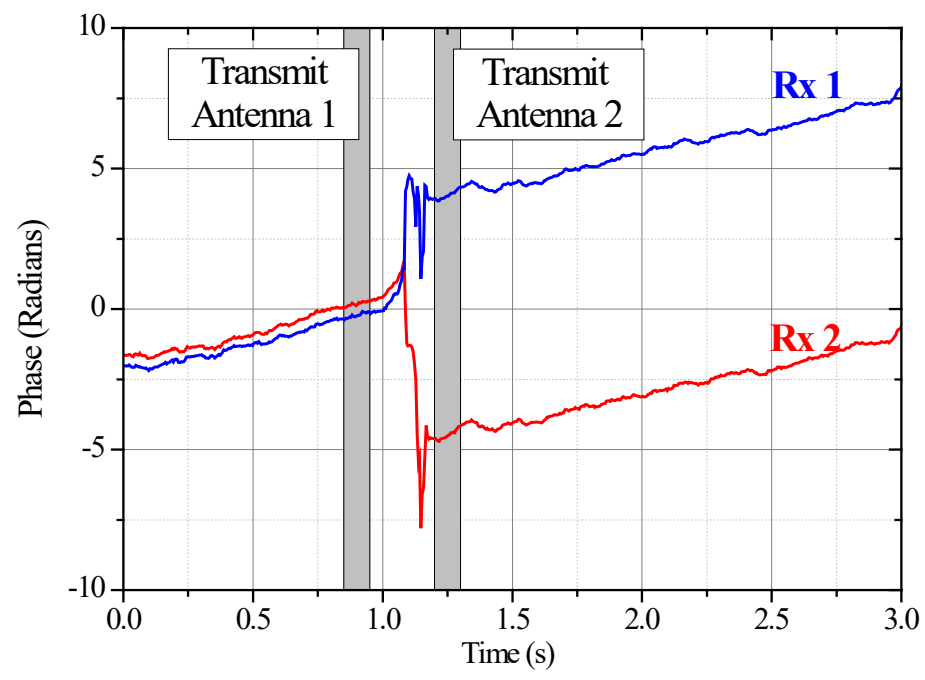

b)

Figure 4.17. MIMO measurement and the switching event, a) amplitude and b) phase variations.

In Figure 4.17a the received signal amplitudes in un-calibrated voltage units are shown. For obtaining the respective MIMO phase and amplitude, two intervals of $100 \mathrm{~ms}$ are manually selected before and after the switching (represented as gray areas). First, the exact frequencies at a constant phase for the two $100 \mathrm{~ms}$ intervals are derived. In Figure 4.17b, it is seen that the phase is almost linear in the gray zones for both channels.

Once the exact frequencies are known, each selected $100 \mathrm{~ms}$ portion of the signal is multiplied by a sine and a cosine function with the same duration. Later, the sum of each product signal is obtained and represented in an I-Q diagram, from which the amplitude and phase are computed. From the resulted complex numbers, the $\mathbf{H}$ matrix is generated: $h_{11}$ and $h_{21}$ are obtained when the first transmitter is on and $h_{12}$ and $h_{22}$ are obtained after switching the transmission to the second transmitter. 


\subsection{Conclusions}

The main characteristics of the experimental equipment and data processing have been presented. For the satellite propagation analyses, several ancillary instruments are used to derive information that allows describing and understanding better the received signal characteristics. Then it is possible, for example, to correlate the rain events in the receiving site with some deep fades in the received beacon signals or, on the other hand, to assume that rain takes place along the path when fades appear in the signal but there is no rain registered at the receiving site. Also, the ancillary instruments have different degrees of accuracy, being possible a better description of a rain event with the disdrometer than with the rain gauge. Occasionally, heavy rain events are clearly overestimated by the disdrometer; in those cases the use of rain gauge values is more convenient.

The KA-SAT receiver has been reused from a previous experiment; since then, 5 years of measurements have been collected so far. The beacon receiver has a rain margin of up to 32 $\mathrm{dB}$, a value to have into account in further analyses.

Alphasat, on the other hand, is an experiment completely new, with 4 years of analyzed measurements so far. Since the contribution of gases is not negligible in the Q band, a procedure to derive it from external data is applied. This procedure relies on the use of GNSS information from a nearby ESA station to calculate the gas attenuation. The clouds and tropospheric scintillation contributions to the attenuation are included in the excess attenuation reference extraction process, as well as rain attenuation. The margin of this receiver is of up to $37.8 \mathrm{~dB}$, although measured values close to the margin must be taken with care.

The millimeter-wave indoor equipment is a new acquisition of the Research Group. With it, path loss and MIMO measurements can be obtained. The basis of all measurements is the calibration procedure, which is based on the free space condition (assuming that this occur in a relatively close distance range but in the far-field zone) using the two horn antennas. The derived calibration constant encompasses the attenuation contributions of all the components in the RF chain, being the remaining attenuation attributed to the path loss. When the omnidirectional antenna is used in reception, the difference in gain between the horn and the omnidirectional antenna is removed to obtain comparable results.

MIMO measurements can be gathered for a $2 \times 2$ omnidirectional antenna configuration. They are rigorously speaking two consecutive SIMO measurements taken with the use of two transmit antennas in a sufficiently short lapse of time, so that the channel can be considered as stable for all its duration. MIMO processing allows the generation of the $\mathbf{H}$ matrix from which the singular values can be derived by means of singular value decomposition. 



\section{Satellite link results}

\section{Introduction}

After describing the main theoretical background and the available equipment for satellite propagation experiments in the previous chapters, the main results of the satellite propagation campaigns can be presented. As it was mentioned before, the experimental campaigns encompass 5 years of KA-SAT (from September 2013 to August 2018) and 4 years of Alphasat beacon measurements (from March 2014 to March 2018, excluding March 2017), with a 4-year concurrent period (the Alphasat period). Hereinafter, results with the two beacons are referred as Ka-band results and Q-band results. In some cases, the Ka-band results are limited to the four years concurrent with the Alphasat measurements for comparison purposes.

The obtained first-order (rainfall rate and attenuation distributions) and second-order (fade dynamics and diversity) statistics for both experiments are compared with some available prediction models presented in Chapter 2. A special attention is given to the second-order statistics, for which a modeling effort has been carried out using the distribution combinations of sub-section 2.5.1 for fade and inter-fade duration results. Also, fade slope results are presented and compared with the ITU-R model.

TD and OD are the diversity techniques treated here. In order to compare their performances, only the concurrent 4-year period is used regarding these techniques. TD results are presented and compared with the previously presented models (Matricciani TD, Joint Probability, Greece and ONERA). OD is assessed by applying a frequency scaling procedure to the KA-SAT results in order to have the necessary data for the two links at the 
same frequency. Diversity results are also compared with the models addressed in Chapter 2 (Matricciani OD and NTUA).

Regarding variability, it is assessed for first- and second-order statistics by using the methods outlined in section 2.7. Rain and attenuation variability are evaluated either for the four or the five years of measurements of the two beacons. On the other hand, fade dynamics variability is only assessed for the 4-year concurrent period for comparison purpose.

Results are grouped by topics rather than by experiments, allowing comparisons in a more natural way.

\subsection{Rainfall rate results}

Rainfall rate distributions are first order statistical results that allow a better understanding of the future analyses. The distributions for the individual years and the period are shown in Figure 5.1 for the 5 -year period coincident in time with the Ka-band measurements (from September 2013 to August 2018) and the 4-year period coincident with the Q-band measurements (from March 2014 to March 2018, excluding March 2017).

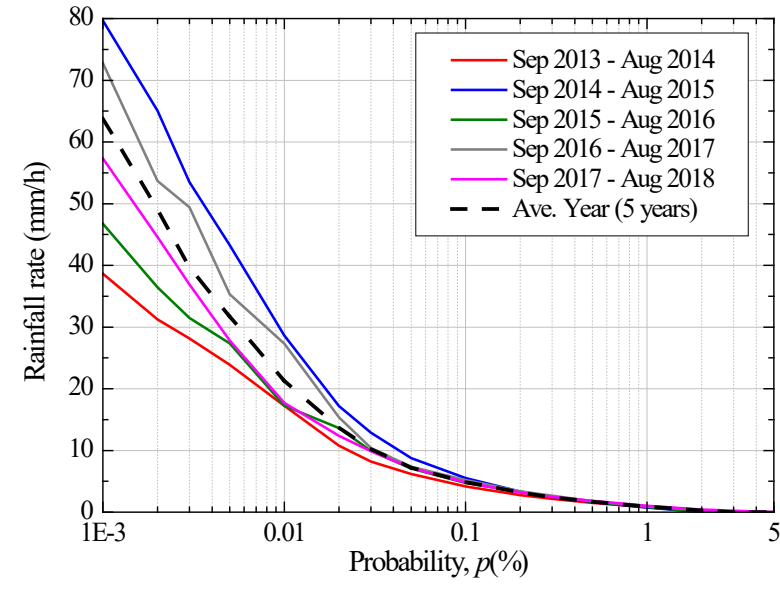

a)

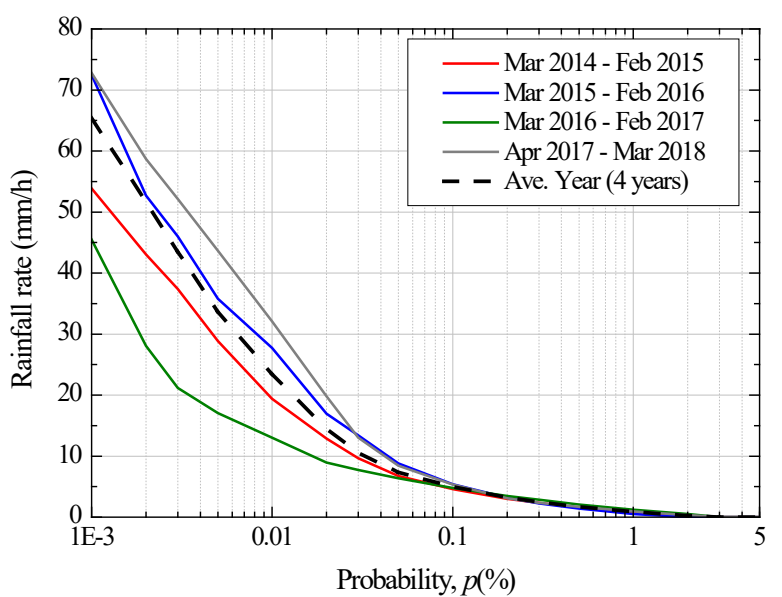

b)

Figure 5.1. Rainfall rate distributions for a) a 5-year period coincident with the Ka-band experiment (from September 2013 to August 2018) and for b) a 4-year period coincident with the Q-band experiment (from March 2014 to March 2018, excluding March 2017).

A high year-to-year variability is seen for probabilities lower than approximately $0.05 \%$ in both cases, meaning that there is high difference between the amounts of convective events from one year to another. Despite the differences between the individual years, the two obtained average-year distributions (Ave. Year in each figure) are very similar.

Figure 5.2 shows a graphical comparison of the rainfall rate distributions of the average years for both experiments with the models that were presented in sub-section 2.3.3. 


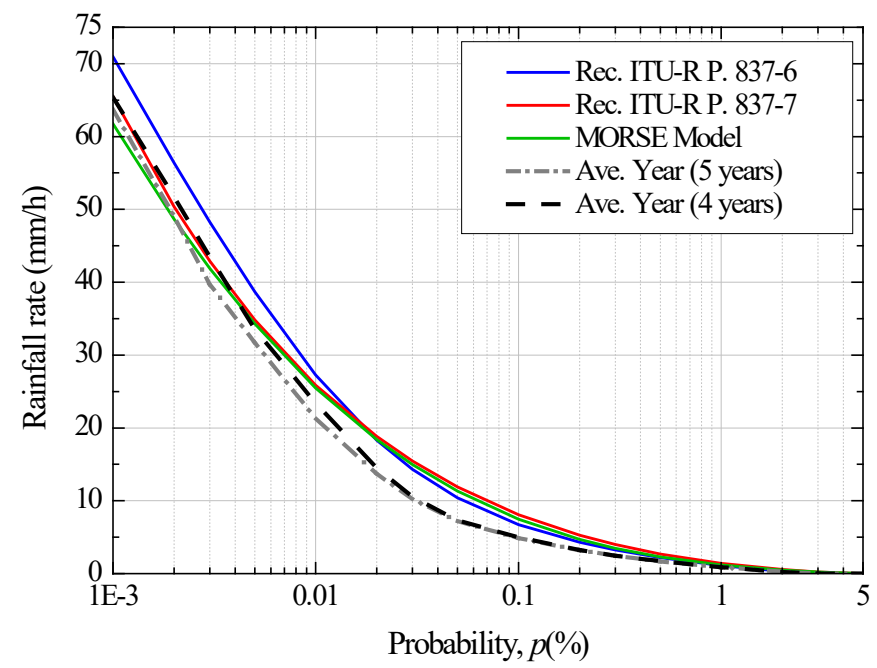

Figure 5.2. Average rainfall rate distributions compared with models.

The three models behave quite similarly, with the ITU-R Rec. P.837-7 and MORSE models giving the best approximations for lower probability values. All models overestimate the rain intensity for medium probabilities (i.e., from approximately $0.005 \%$ to $0.5 \%$ ). Table 5.1 collects the RMS, the standard deviation (Std.) and the mean $(m)$ values of the errors associated to each model, for probabilities of up to $2 \%$, derived by using the ITU-R testing variable for rainfall rate predictions $[\mathbf{2 2 1}]$ :

$$
\varepsilon(p)=\frac{R_{p}(p)-R_{m}(p)}{R_{m}(p)}
$$

with $R_{p}(p)$ and $R_{m}(p)$ being the predicted and measured rainfall rate values for probabily $p$, respectively. Also, the percentage probabilities of rain in an average year $\left(P_{0}\right)$ derived from each $R_{p}(p)$ are given; they can be compared with the measured values of $2.22 \%$ for the 4 year period and $2.4 \%$ for the 5-year period. Overall, the ITU-R Rec. P.837-6 model gives the best results.

Table 5.1. Statistical values of the rainfall rate model (up to $2 \%$ probability)

\begin{tabular}{l|ccc|cc|cc}
\hline \hline \multirow{2}{*}{ Models } & \multirow{2}{*}{$\boldsymbol{P}_{\mathbf{0}}$} & \multicolumn{2}{|c|}{$\boldsymbol{R M S} \mathbf{( \% )}$} & \multicolumn{2}{c|}{$\boldsymbol{m}$} & \multicolumn{2}{c}{ Std. } \\
& & 4 years & 5 years & 4 years & 5 years & 4 years & 5 years \\
\hline Rec. ITU-R P.837-6 & 2.72 & 26.90 & 29.47 & 24.81 & 27.74 & 10.78 & 10.33 \\
Rec. ITU-R P.837-7 & 3.99 & 59.09 & 55.22 & 43.40 & 45.09 & 41.62 & 33.07 \\
MORSE & 3.12 & 44.29 & 41.33 & 31.46 & 33.36 & 32.36 & 25.31 \\
\hline \hline
\end{tabular}

\subsection{Excess Attenuation results}

Only excess attenuation results and analyses derived from them are given in this chapter. The reason to use excess instead of total attenuation is that the majority of the applied models uses such kind of data, thus the comparisons and analyses can be carried out directly. Total attenuation has been calculated only for Q-band (since gas attenuation in the Ka-band is less relevant and can be neglected) and results are presented in Annex 3, avoiding duplications in the analyses, since they are very similar to excess attenuation results. 
Figure 5.3 shows the yearly excess attenuation distributions for each experiment, together with the average-year distribution derived from them. Here, the successive years have been referenced with a number. Then, for the 5-year period, Year 1 corresponds with September 2013 to August 2014, Year 2 with September 2014 to August 2015, Year 3 with September 2015 to August 2016, Year 4 with September 2016 to August 2017 and Year 5 with September 2017 to August 2018. In the 4-year case, Year 1 stands for March 2014 to February 2015, Year 2 for March 2015 to February 2016, Year 3 for March 2016 to February 2017 and Year \& for April 2017 to March 2018.

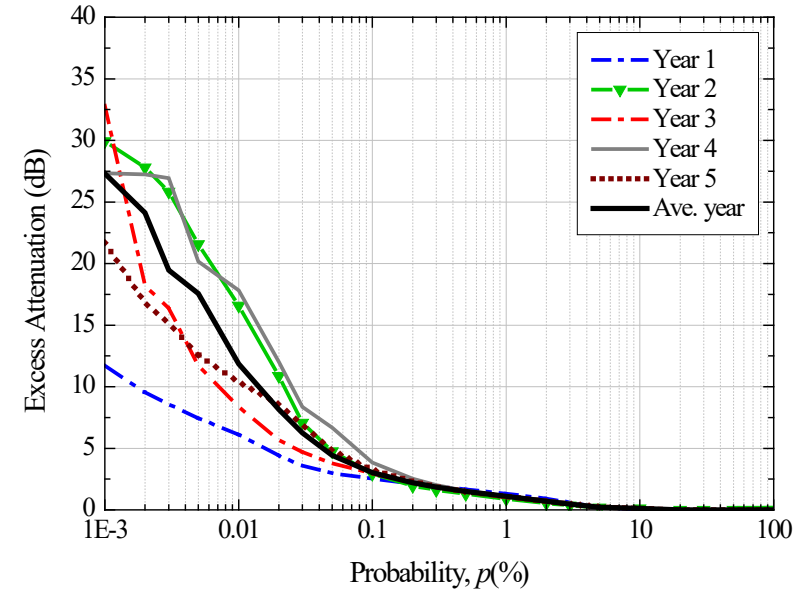

a)

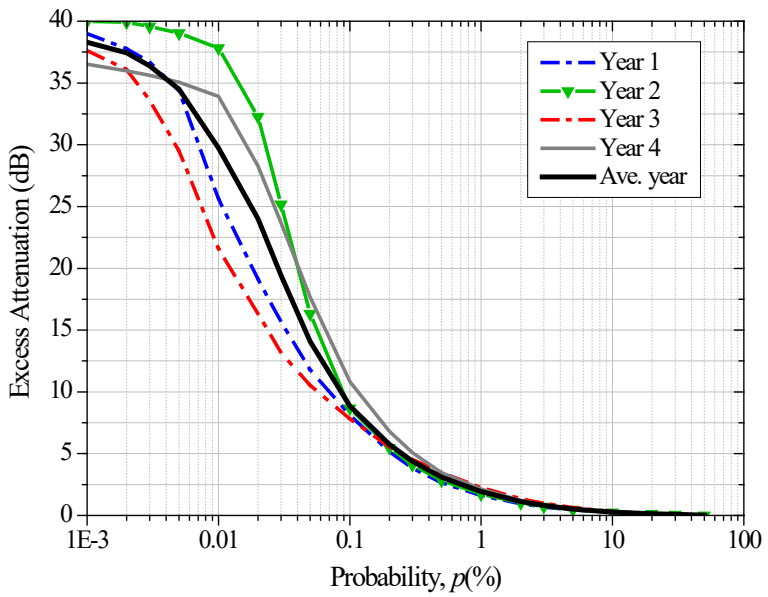

b)

Figure 5.3. Attenuation distributions for the individuals and average year for a) Ka- and b) Q-band.

In Table 5.2, several values of measurement availability regarding both experiments are presented. Total attenuation availability is only given for Q-band, since it has been collected only for that experiment.

Excess and total attenuation availabilities are calculated as the quotient of the number of valid samples per year (or period) to the total number of samples for the entire year (or period).

Table 5.2. Data availability (in \%)

\begin{tabular}{ccccccc}
\hline \hline Ka-band & Year 1 & Year 2 & Year 3 & Year 4 & Year 5 & Period \\
\hline Excess attenuation & 98.10 & 97.37 & 98.09 & 99.09 & 97.11 & 97.95 \\
\hline Q-band & & & & & & \\
\hline Excess attenuation & Year 1 & Year 2 & Year 3 & Year 4 & Period \\
\hline Total attenuation & 97.40 & 96.03 & 98.61 & 98.67 & 97.61 \\
\hline \hline
\end{tabular}

Total attenuation availability is usually lower than Excess attenuation availability because it depends not only on the availability of measurements but also on the availability of GNSS data used to derive the gas attenuation (as was explained in sub-section 4.1.3.3, the difference between total and excess attenuation is the gas attenuation, derived here by means of the procedure of $[\mathbf{2 1 0}])$. 


\subsubsection{Ka-band distributions}

The monthly and annual distributions for the Ka-band are displayed in Figure 5.4. Also, the Worst Month (the month in a period of twelve consecutive calendar months, during which a threshold is exceeded for the longest time [222]) distributions for each year are included.

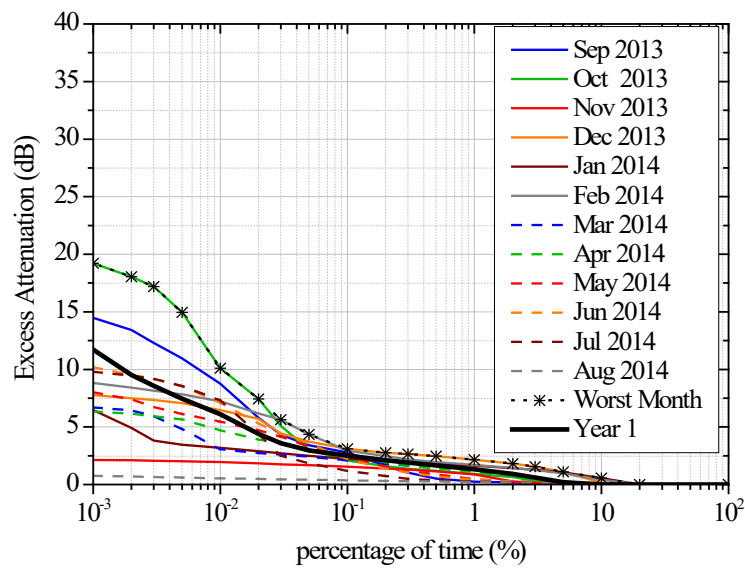

a)

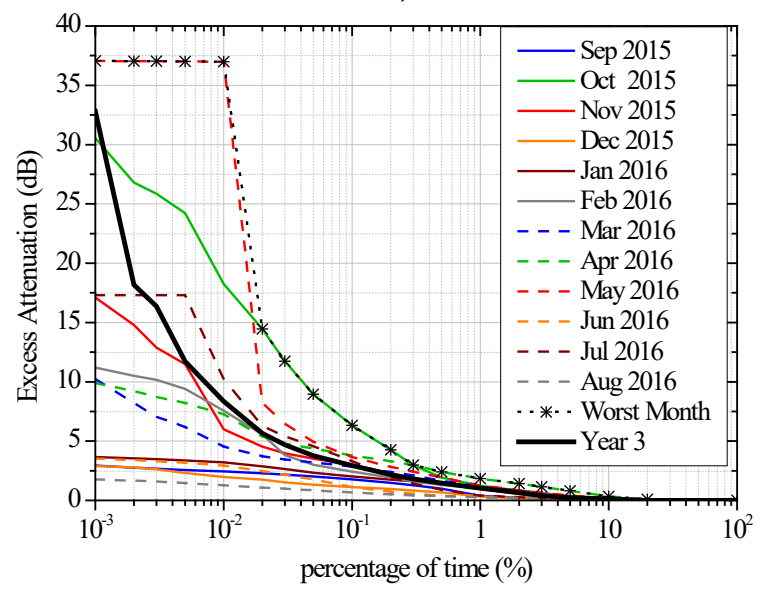

c)

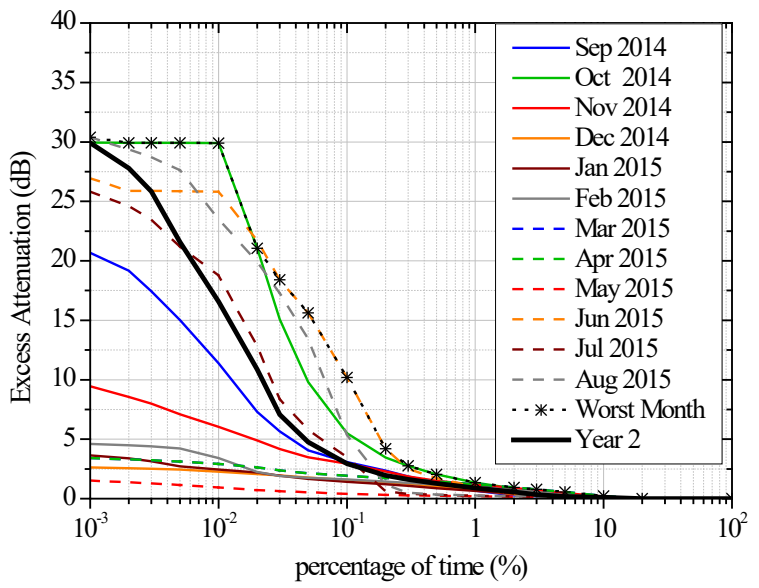

b)

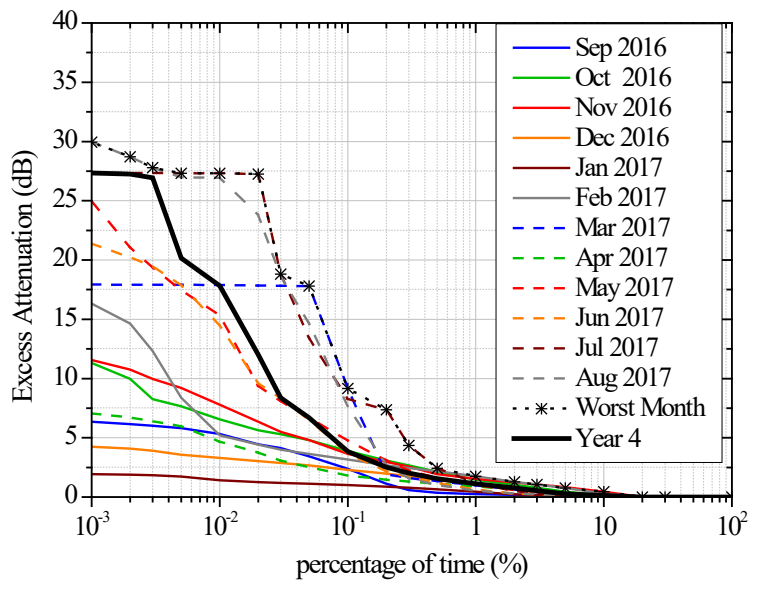

d)

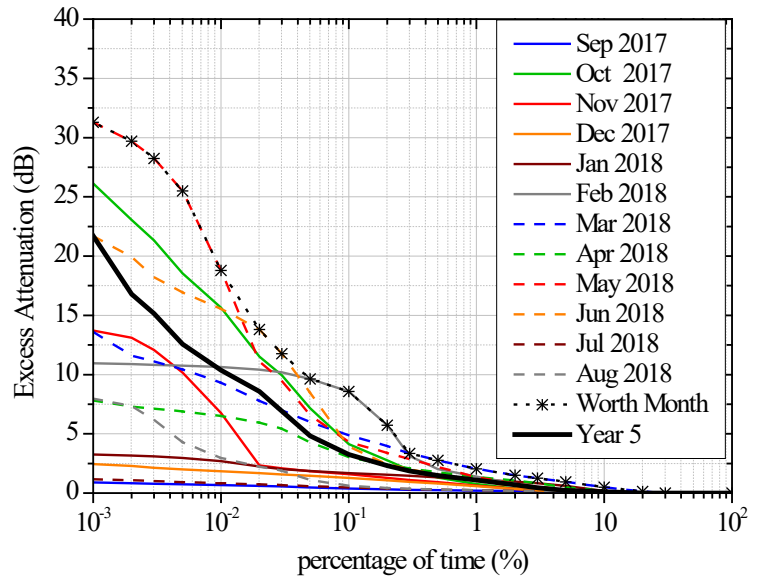

e)

Figure 5.4. Ka-band monthly (including the worst month) and annual excess attenuation distributions. 
From these results a high month-to-month variability among the years is observed. For example, October is the month when most of the convective events occur for Year 1 and 2, but in Year 4 it is a rather average month.

It is necessary to bear in mind that the margin for this experiment is around $32 \mathrm{~dB}$, and then the values obtained for lower probabilities for some months (such as Oct 2014, Nov 2015, etc.) are seen as flattened in the attenuation curve due to the saturation of the receiver dynamic range. This behavior is also observed in Year 4.

\subsubsection{Q-band distributions}

The monthly (including the worst month) and annual distributions for the Q-band are displayed in Figure 5.5.

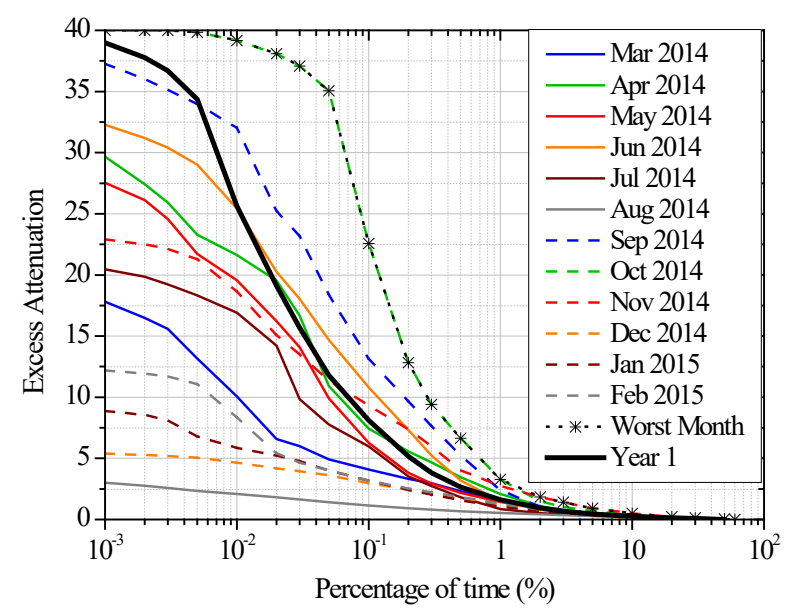

a)

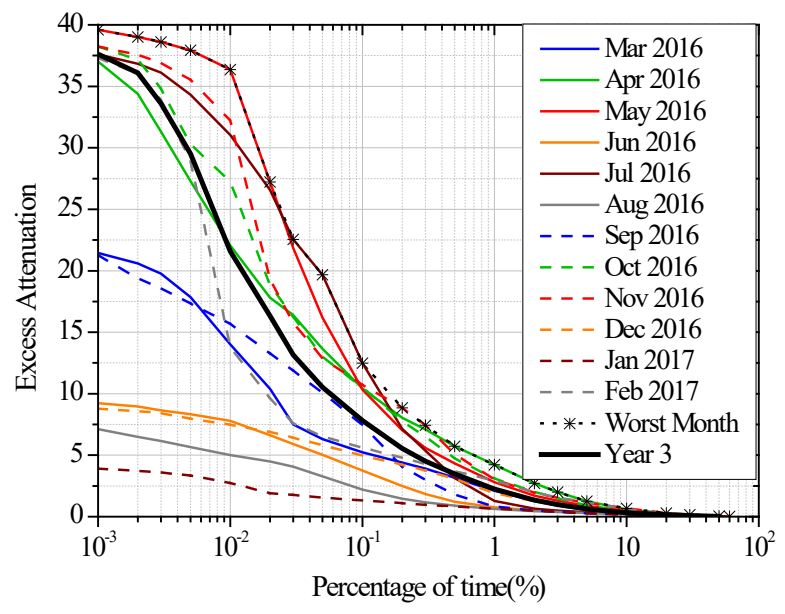

c)

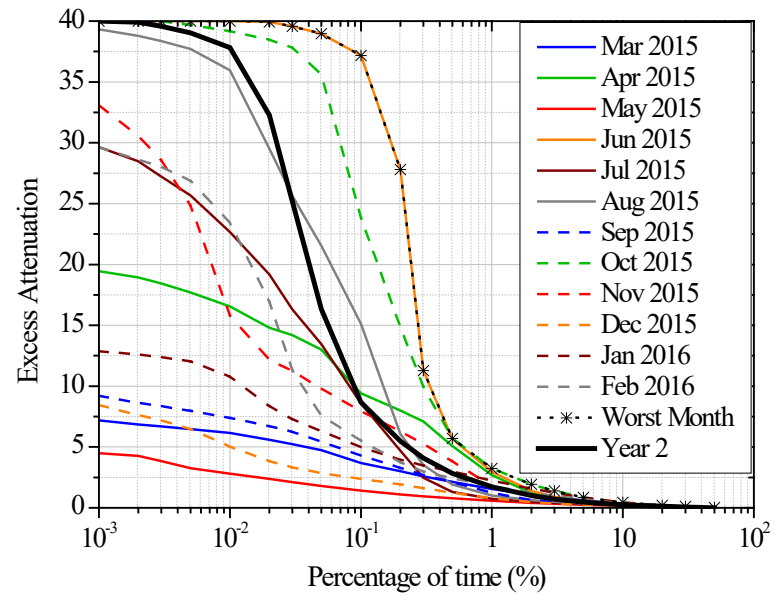

b)

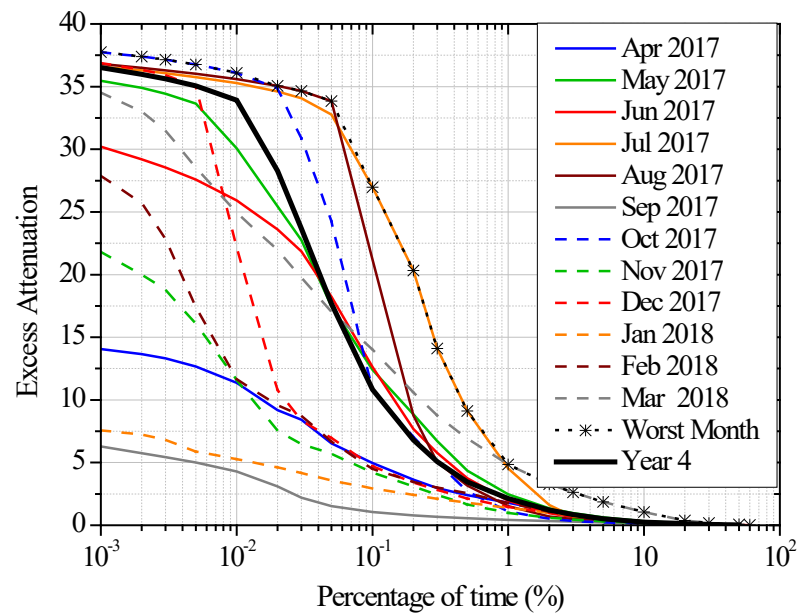

d)

Figure 5.5. Q-band monthly (including the worst month) and annual excess attenuation distributions.

There are no measurements of March 2017 due to a malfunction in the receiver unit; this is the reason to start Year 4 in April 2017 instead of March. It can be seen that April and June were the months that contribute the most to the worst month statistics for Year 1 and Year 2 respectively. For Years 3 and 4, the higher attenuation events are more distributed 
among several months. The saturation of the dynamic range of the receiver (up to approximately $38 \mathrm{~dB}$ ) is seen here as a smoothness of the curves near that value.

\subsection{Fade dynamics results}

\subsubsection{Fade duration results}

From the excess attenuation time series for the two campaign periods, a fade duration (FD) analysis has been carried out, and its results are compared with the Rec. ITU-R P.1623-1 and CRC prediction methods.

Since the ITU-R model is intended to be used with total attenuation data, the estimated gas attenuation for the period was summed in its thresholds, e.g. a 3dB+average-gasattenuation threshold of the ITU-R model is analogous to the $3 \mathrm{~dB}$ curve of excess attenuation data - although for a matter of simplicity, all the figures will display the attenuation thresholds $A_{\text {th }}$ of $3,5,10$ and $15 \mathrm{~dB}$. The average gas attenuation value is found by using the procedure of $[\mathbf{2 1 0}]$ and it is approximately $0.7 \mathrm{~dB}$ for the Q-band experiment. This procedure was not applied to the Ka-band results because of the low value of gas attenuation at that frequency, being possible to neglect its contribution to the total attenuation, which is not the case in the Q-band.

For assessing the possible improvements to the prediction methods, a fitting effort has been conducted. The performances of the fitting and models are accounted for by using the testing variable of (5.2), recommended by the ITU-R [221] for the fade duration probability of occurrence:

$$
\varepsilon_{n}\left(D \mid A_{t h}\right)=100 \cdot \ln \left(\frac{P_{\text {pred }}\left(D \mid A_{t h}\right)}{P_{\text {meas }}\left(D \mid A_{t h}\right)}\right)
$$

with $P_{\text {pred }}\left(D \mid A_{t h}\right)$ and $P_{\text {meas }}\left(D \mid A_{t h}\right)$ being the predicted (by the models or the fitting) and the experimental probabilities of occurrence for the given duration and attenuation threshold, respectively.

In the case of the total fraction of fading time, the testing variable recommended by [221] is used:

$$
\varepsilon_{t}\left(D \mid A_{t h}\right)=100 \cdot \ln \left(\frac{1-F_{\text {pred }}\left(D \mid A_{t h}\right)}{1-F_{\text {meas }}\left(D \mid A_{\text {th }}\right)}\right)
$$

with $F_{\text {pred }}\left(D \mid A_{t h}\right)$ and $F_{\text {meas }}\left(D \mid A_{t h}\right)$ being the predicted (by the models or the fitting) and the experimental fraction of fading time for the given duration and attenuation threshold, respectively.

\subsubsection{Comparison with models}

The experimental distributions of the number of fades and fading time are compared with the ITU-R predictions in Figures 5.6 and 5.7 respectively. The experimental values of the total number of fades and total fading time for each threshold were used to derive the ITU-R 
estimates (basically this works as a normalization of the ITU-R curves to the first experimental value).

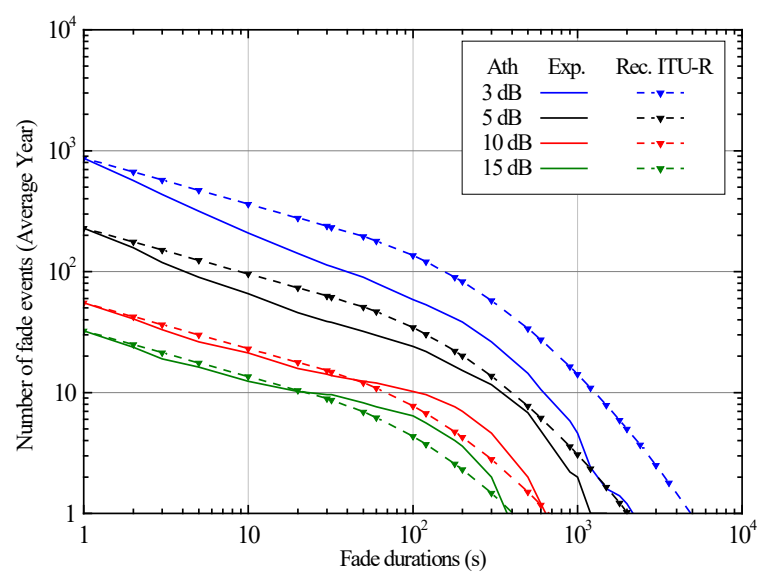

a)

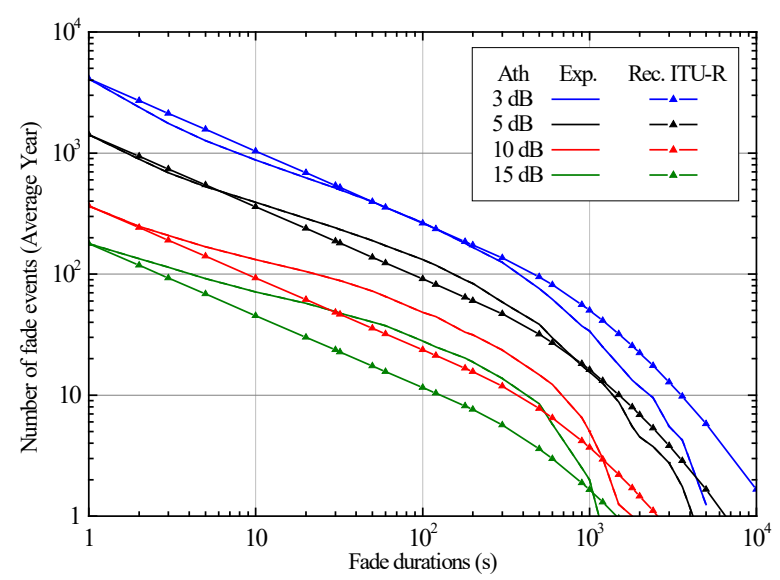

b)

Figure 5.6. FD Number of fade events compared with the ITU-R model for the a) Ka- b) and Q-band.

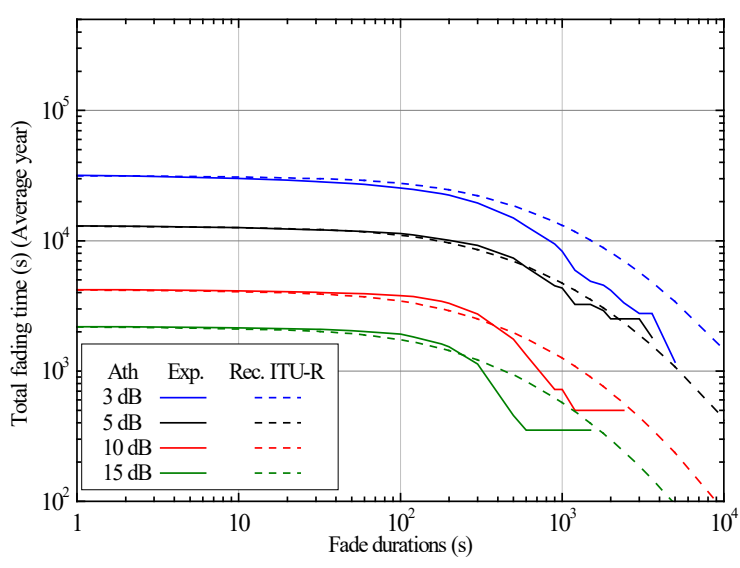

a)

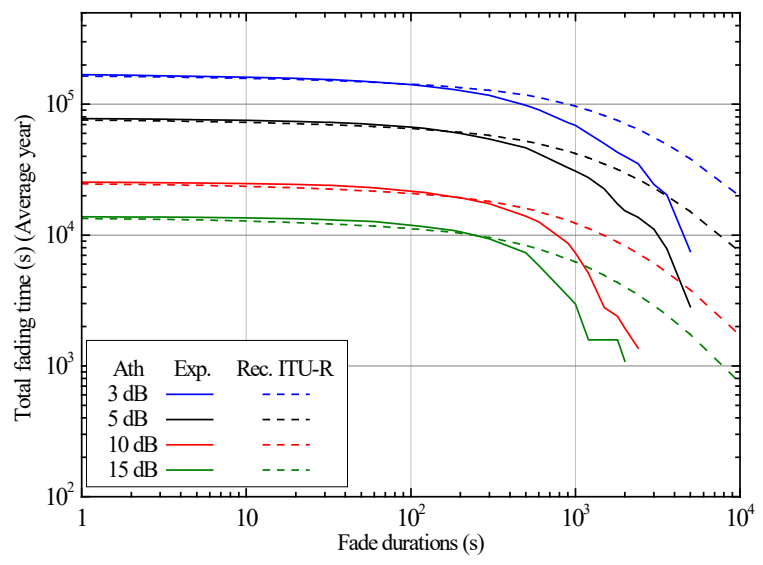

b)

Figure 5.7. FD Total fading time compared with the ITU-R model for the a) Ka- b) and Q-band.

For the number of fade events, the ITU-R predictions are very good for the 10 and $15 \mathrm{~dB}$ curves for Ka-band and for the 3 and $5 \mathrm{~dB}$ curves in the case of Q-band. The total fading time has a very good approximation up to approximately $300 \mathrm{~s}$ for both cases.

The experimental FD probability of occurrence distributions are derived and graphically compared with the ITU-R and CRC models in Figure 5.8. The distributions of the fraction of fading time (derived from the total fading time) are compared with the ITU-R model in Figure 5.9. 


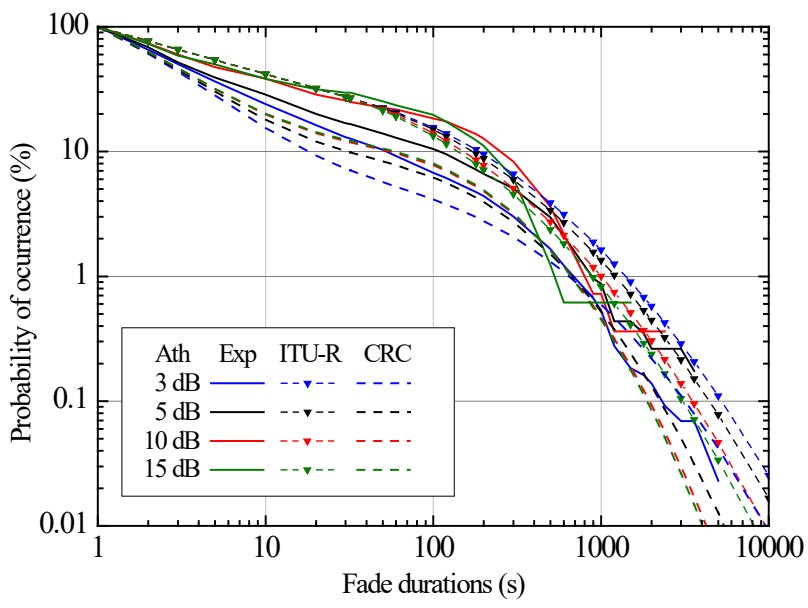

a)

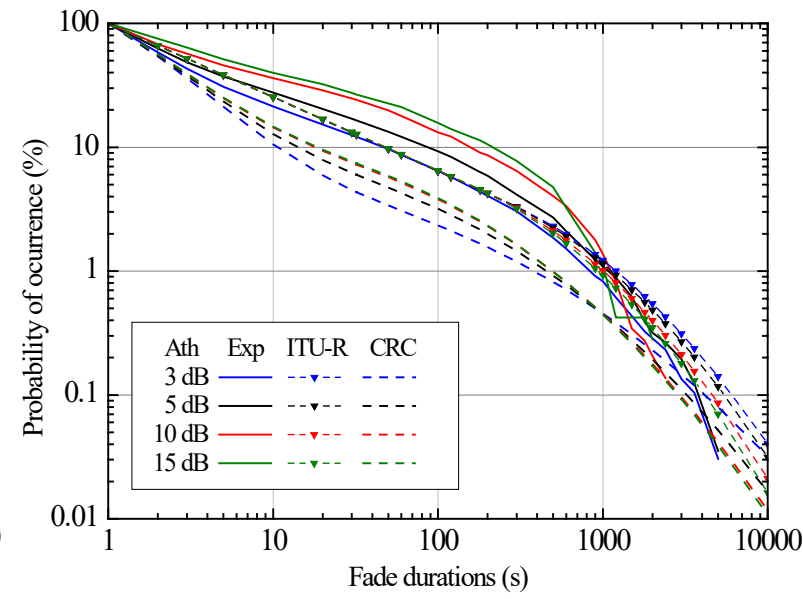

b)

Figure 5.8. FD probability of occurrence compared with the CRC and ITU-R models for a) Ka- and b) Q-band.

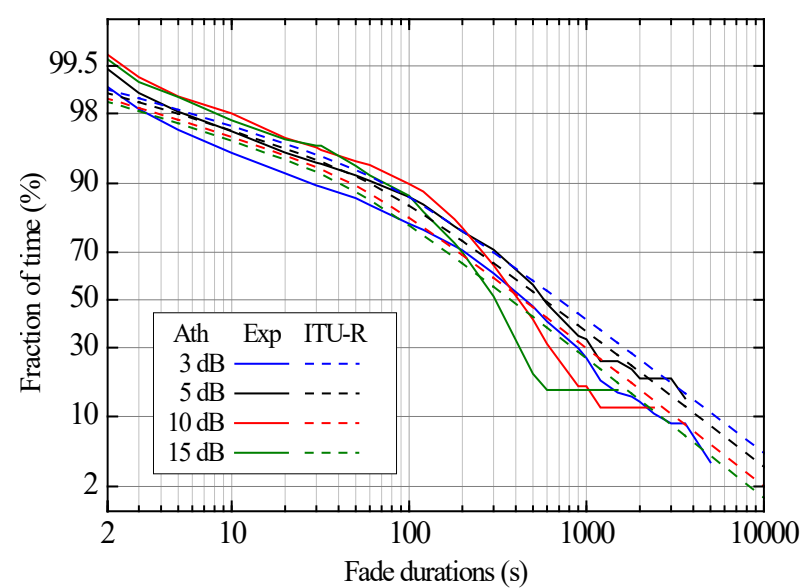

a)

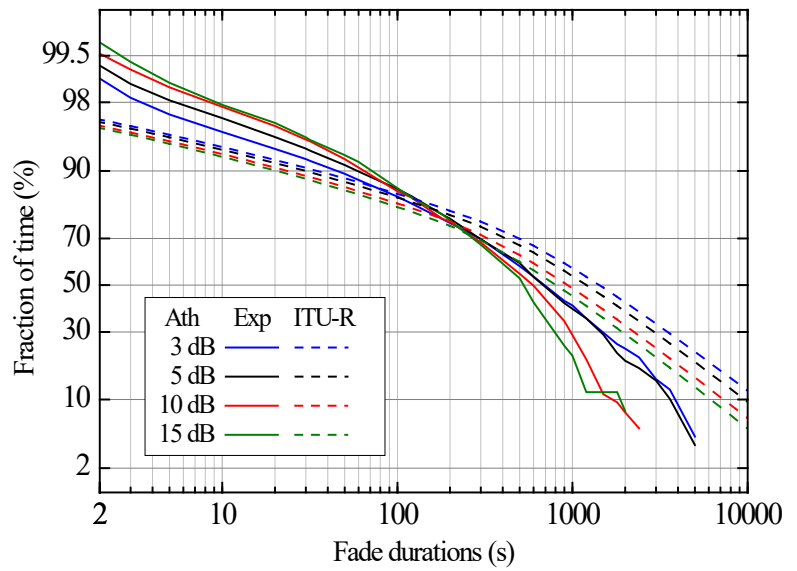

b)

Figure 5.9. FD fraction of fade time compared with the ITU-R model for a) Ka- and b) Q-band.

For the probability of occurrence, the CRC curves largely deviate from the Q-band results. In the case of the fraction of fading time, the ITU-R model predictions follow the trends of the experimental curves better for the Ka-band than for the Q-band results. Also, the model underestimates the Q-band results for fade duration shorter than approximately $150 \mathrm{~s}$ and overestimates them for longer durations.

Table 5.3 compares the models by using the testing variables defined by (5.2) and (5.3), depending on the case. 
Table 5.3. FD RMS, standard deviation (Std.) and mean values of the testing variables used to compare the experimental results vs. models

\begin{tabular}{|c|c|c|c|c|c|c|c|c|c|}
\hline \multirow{3}{*}{$\begin{array}{l}\text { Attenuation } \\
\text { level }\end{array}$} & \multicolumn{6}{|c|}{$\begin{array}{l}\text { Testing Variable for the FD } \\
\text { Probability of Occurrence (\%) }\end{array}$} & \multirow{2}{*}{\multicolumn{3}{|c|}{$\begin{array}{c}\text { Testing Variable for Total } \\
\text { Fraction of Fading Time (\%) } \\
\text { ITU-R Model }\end{array}$}} \\
\hline & \multicolumn{3}{|c|}{ ITU-R Model } & \multicolumn{3}{|c|}{ CRC Model } & & & \\
\hline & RMS & Std. & Mean & RMS & Std. & Mean & RMS & Std. & Mean \\
\hline \multicolumn{10}{|c|}{ Ka-band } \\
\hline $3 \mathrm{~dB}$ & 88.25 & 40.12 & 79.06 & 44.45 & 39.37 & -22.20 & 28.64 & 21.51 & -19.20 \\
\hline $5 \mathrm{~dB}$ & 51.74 & 21.47 & 47.29 & 38.78 & 15.51 & -35.69 & 12.13 & 12.21 & -1.35 \\
\hline $10 \mathrm{~dB}$ & 39.37 & 40.36 & -2.64 & 71.47 & 39.88 & -60.02 & 35.92 & 34.34 & 11.80 \\
\hline $15 \mathrm{~dB}$ & 42.88 & 41.95 & -13.74 & 80.05 & 49.77 & -63.92 & 38.80 & 37.66 & 11.00 \\
\hline RMS Ave. & 55.56 & & & 58.68 & & & 28.87 & & \\
\hline \multicolumn{10}{|c|}{ Q-band } \\
\hline $3 \mathrm{~dB}$ & 29.73 & 22.01 & 20.50 & 75.26 & 35.59 & -66.72 & 28.91 & 29.16 & -2.42 \\
\hline $5 \mathrm{~dB}$ & 23.96 & 22.59 & -9.27 & 87.10 & 32.96 & -80.93 & 41.11 & 40.74 & 8.34 \\
\hline $10 \mathrm{~dB}$ & 54.83 & 44.46 & -33.39 & 103.6 & 47.63 & -92.54 & 57.81 & 56.36 & 15.54 \\
\hline $15 \mathrm{~dB}$ & 62.58 & 44.33 & -45.13 & 111.3 & 48.48 & -100.67 & 67.19 & 64.63 & 20.90 \\
\hline RMS Ave. & 42.78 & & & 94.32 & & & 48.76 & & \\
\hline
\end{tabular}

For the probability of occurrence, the ITU-R model gives the best overall estimates, and the CRC model approximates better the Ka-band than the Q-band results. Maybe the CRC behavior is related to the fact that most of the campaigns used in the development of this model were performed in the Ka-band and only a reduced amount were carried out at higher frequencies. For the fraction of fading time, the predictions are better for the Ka-band.

\subsubsection{FD probability of occurrence modeling}

The four distribution combinations of sub-section 2.5.1 were used for fitting the experimental probability of occurrence distributions, applying for the first three ( $\log +\log$, Exp+Log, Pwr+Log) the ONERA fitting procedure $[\mathbf{1 1 2}]$ and for the three-decreasing exponentials (Exp+Exp+Exp) the one used by Bråten [109].

A better look at Figure 5.7 for the Ka-band case shows that, for the 10- and 15-dB curves, there are several probabilities with the same total fading time (seen as a parallel line in the tail of the distributions). This is not seen for the number of events because the graphic starts at 1 , then fractions of events are not shown (a fraction of event can be obtained if only an event of certain long duration occurs in the entire period). These flat parts in the tails are seen again for the probability of occurrence and the fraction of fading time. Since this part of the curves may be difficult to model and only represent fractional events with very little importance from a practical perspective, due to their low probability of occurrence, the fitting effort has been carried out in all cases only for the part of the curves that do not show that trend.

Figures 5.10 and 5.11 show, in the left-hand plots, the probability of occurrence and the curves obtained from the fitting effort for different thresholds for each experiment. In the right-hand plots, the testing variables of (5.2) are shown for each case. 

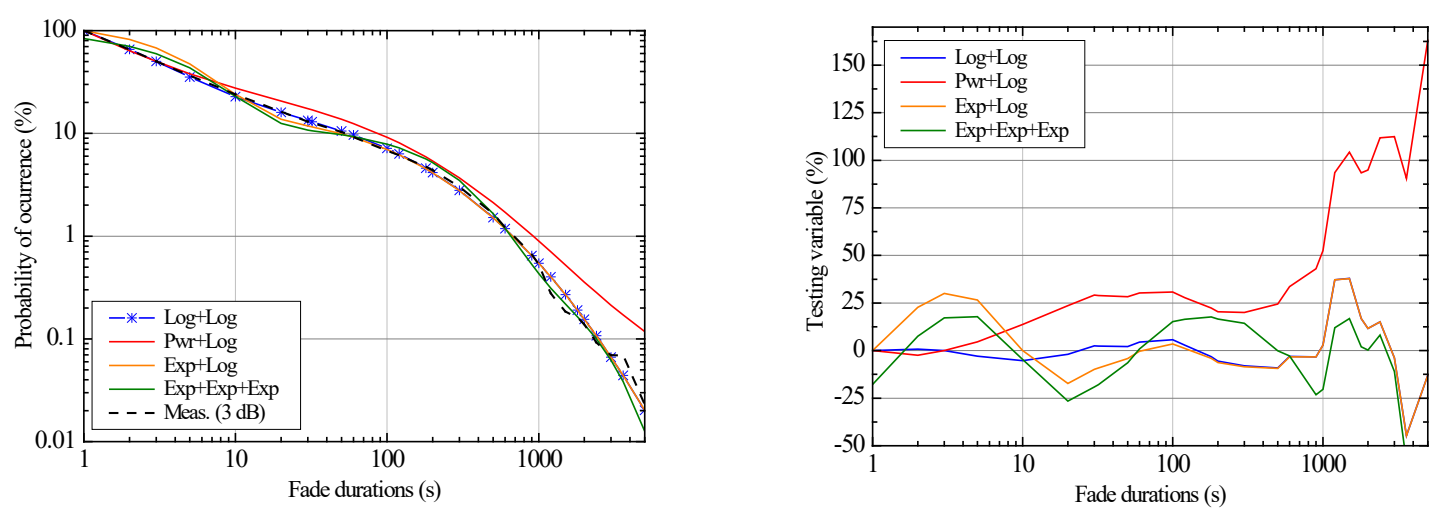

a)
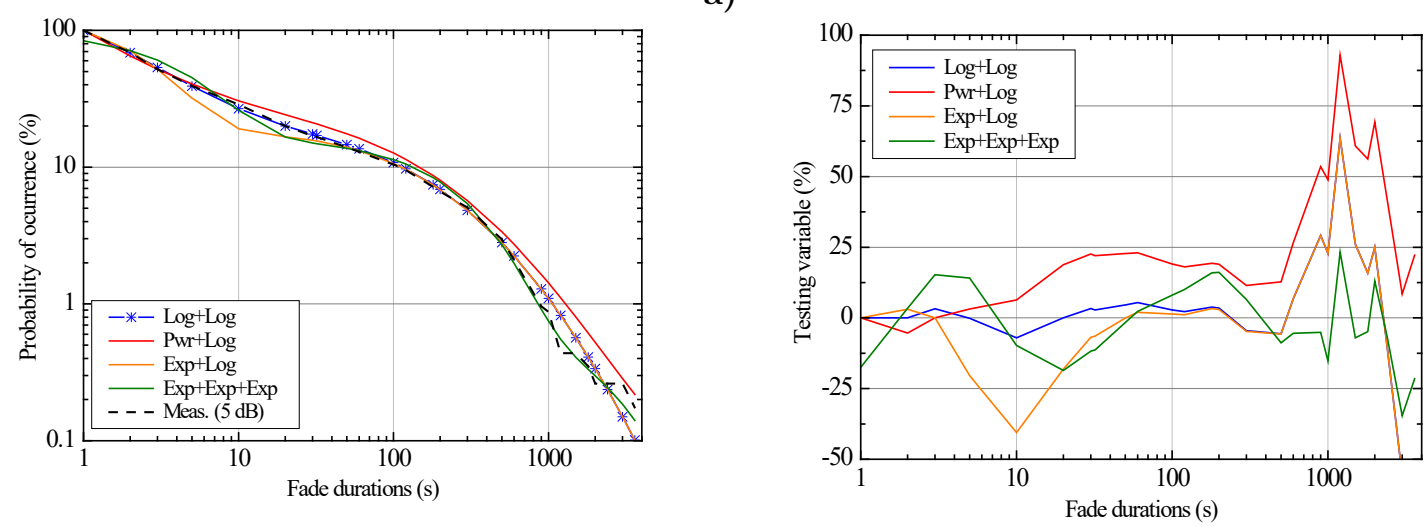

b)
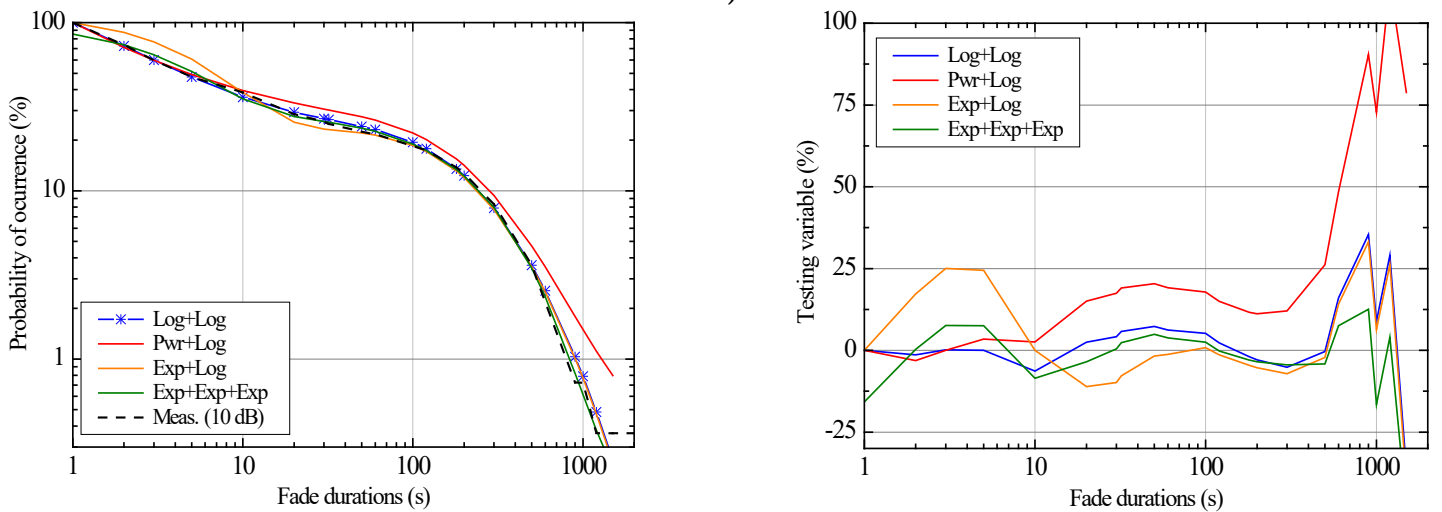

c)
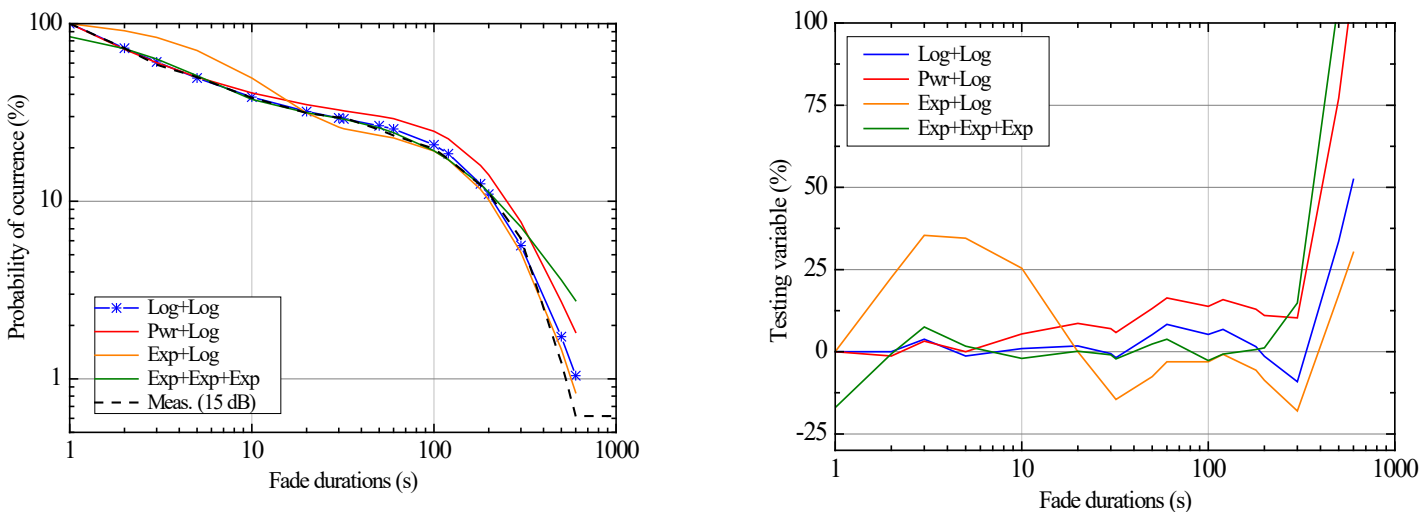

d)

Figure 5.10. Ka-band FD probability of occurrence results and fitting procedure graphs (left-hand column) and testing variable (right-hand column) for $A_{t h}$ equal to a) $3 \mathrm{~dB}$, b) $5 \mathrm{~dB}$, c) $10 \mathrm{~dB}$, d) $15 \mathrm{~dB}$. 

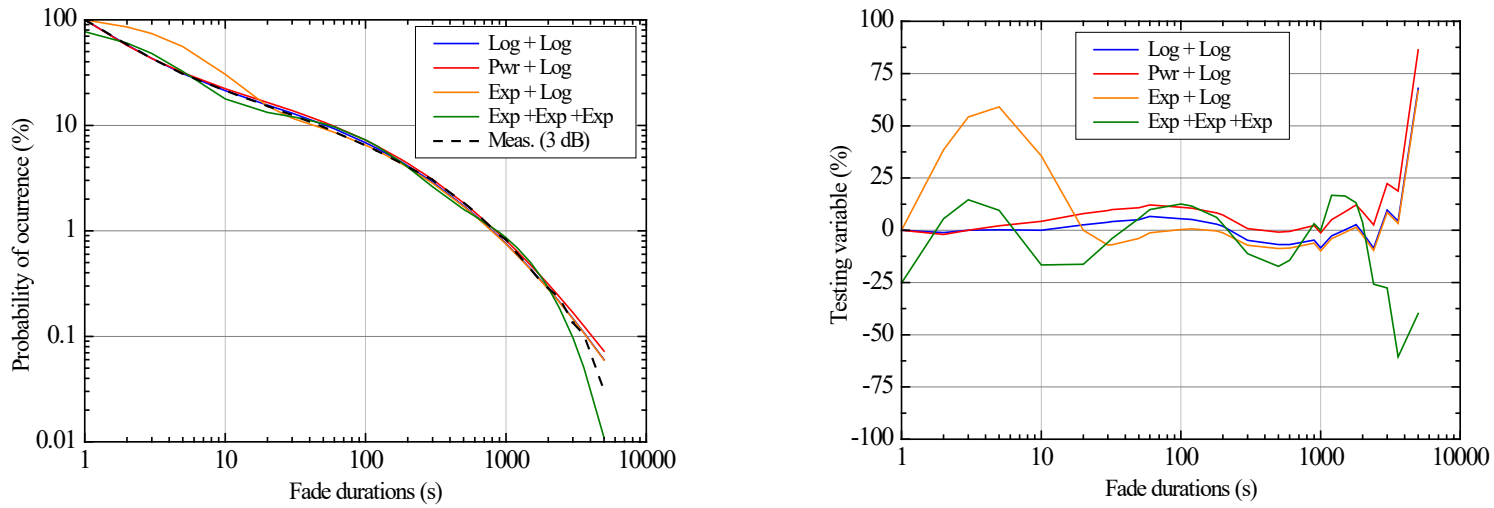

a)
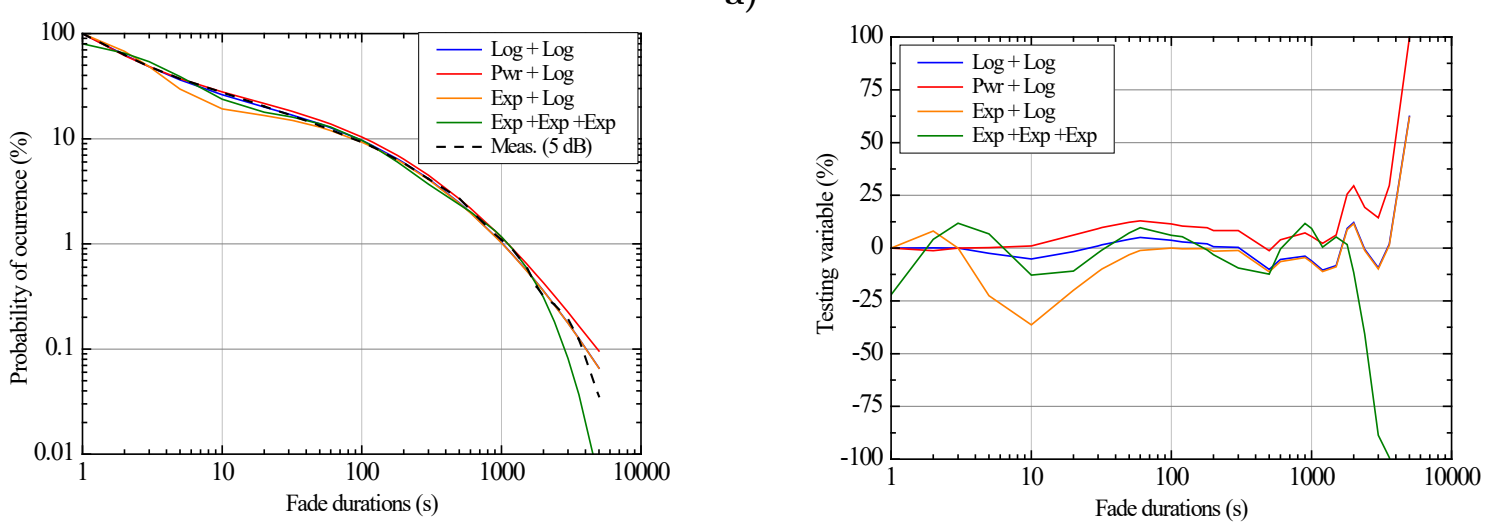

b)
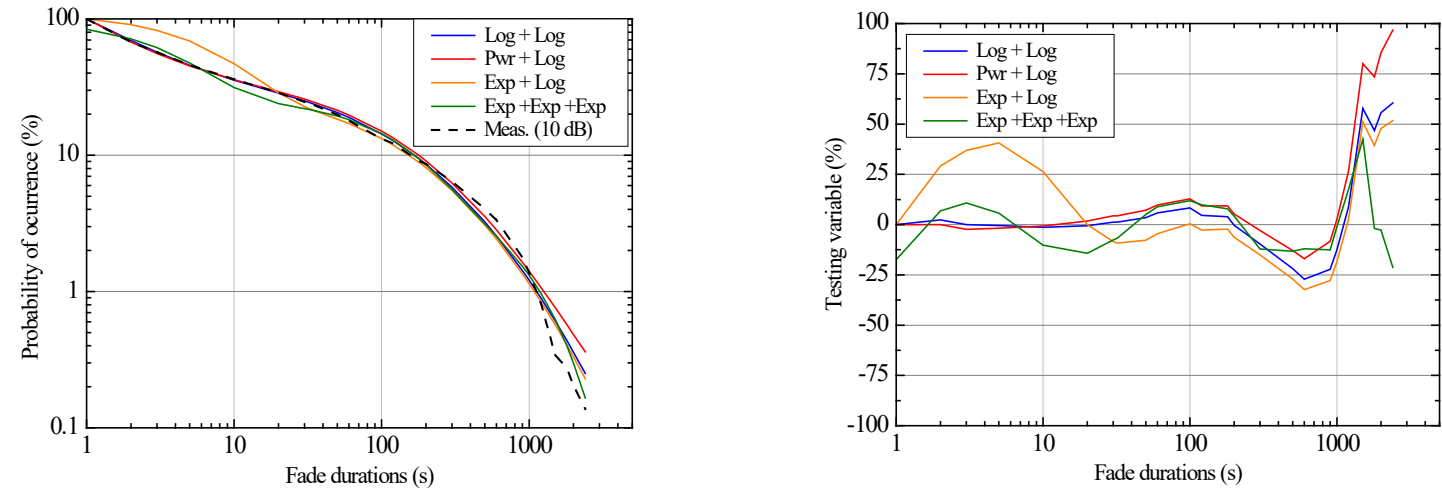

c)
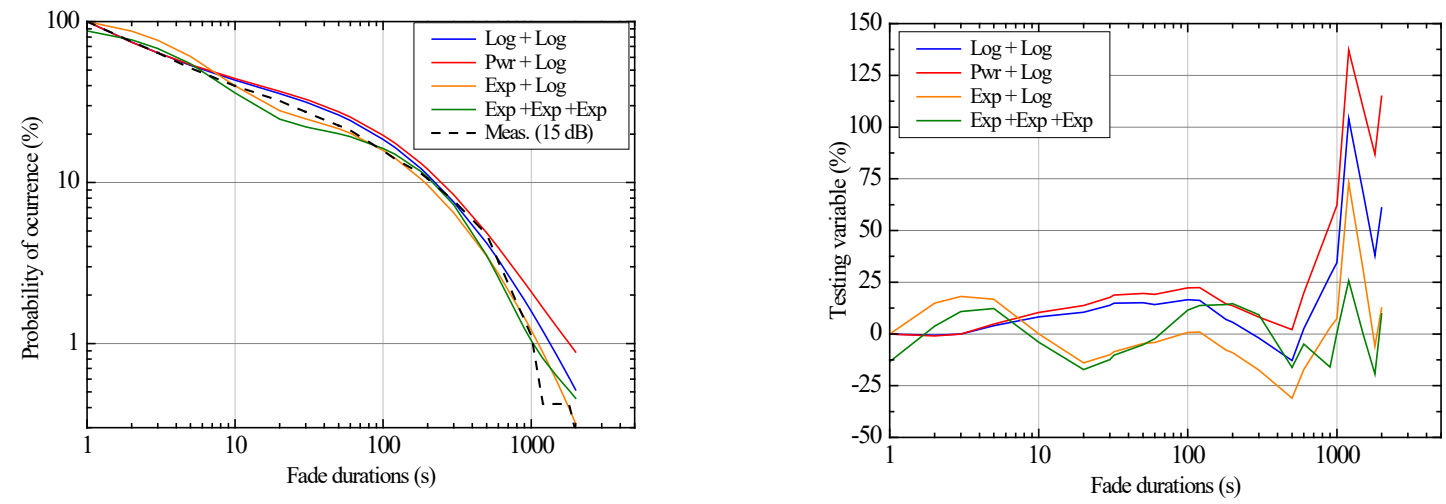

d)

Figure 5.11. Q-band FD probability of occurrence results and fitting procedure graphs (left-hand column) and testing variable (right-hand column) for $A_{t h}$ equal to a) $3 \mathrm{~dB}$, b) $5 \mathrm{~dB}$, c) $10 \mathrm{~dB}$, d) $15 \mathrm{~dB}$. 
Most of the combinations of Figures 5.10 and 5.11 fit the experimental results properly, although higher differences are obtained for longer durations (higher than $1000 \mathrm{~s}$ ) in all the cases. The Exp+Log combination seems to deviate more than the others for shorter fade durations, a trend also seen in [223] for the first three Q-band years.

The RMS values of the testing variables obtained after using (5.2) are provided in Table 5.4. From there, it is clear that the $\log +\log$ combination gives the best results for both experiments. The Exp+Log and the Exp+Exp+Exp combinations give also good results in general. All these values are better than those presented for the models in Table 5.3.

The best-fit parameters of the two-distribution combinations are detailed in Table 5.5 and are given in Table 5.6 for the three exponential combinations.

Table 5.4. Testing variable for the fade duration probability of occurrence

\begin{tabular}{|c|c|c|c|c|c|c|c|c|c|c|c|c|}
\hline \multirow[b]{3}{*}{$A_{t h}$} & \multicolumn{12}{|c|}{ Testing Variable for the FD Probability of Occurrence (\%) } \\
\hline & \multicolumn{3}{|c|}{$\log +\log$} & \multicolumn{3}{|c|}{ Power $+\log$} & \multicolumn{3}{|c|}{$\operatorname{Exp}+\log$} & \multicolumn{3}{|c|}{$\operatorname{Exp}+\operatorname{Exp}+\operatorname{Exp}$} \\
\hline & RMS & Std. & Mean & RMS & Std. & Mean & RMS & Std. & Mean & RMS & Std. & Mean \\
\hline \multicolumn{13}{|c|}{ Ka-band } \\
\hline $3 \mathrm{~dB}$ & 14.89 & 15.10 & 1.44 & 64.07 & 43.28 & 47.97 & 17.77 & 17.99 & 2.53 & 21.44 & 21.49 & -3.92 \\
\hline $5 \mathrm{~dB}$ & 22.74 & 22.97 & 3.18 & 35.48 & 27.87 & 26.67 & 24.65 & 25.12 & 3.18 & 13.92 & 14.05 & -2.00 \\
\hline $10 \mathrm{~dB}$ & 13.81 & 13.76 & 3.26 & 42.54 & 32.71 & 28.12 & 16.22 & 16.41 & 2.62 & 12.77 & 12.81 & -2.62 \\
\hline $15 \mathrm{~dB}$ & 15.64 & 14.81 & 6.20 & 34.36 & 29.95 & 18.34 & 18.30 & 18.03 & 5.38 & 45.03 & 43.60 & 15.45 \\
\hline $\begin{array}{l}\text { RMS. } \\
\text { Ave. }\end{array}$ & 16.77 & & & 44.11 & & & 19.23 & & & 23.29 & & \\
\hline \multicolumn{13}{|c|}{ Q-band } \\
\hline $3 \mathrm{~dB}$ & 13.88 & 13.83 & 2.88 & 18.79 & 16.52 & 9.50 & 23.00 & 23.32 & 7.04 & 19.33 & 19.05 & -4.94 \\
\hline $5 \mathrm{~dB}$ & 13.18 & 13.33 & 1.63 & 22.83 & 19.40 & 12.61 & 16.23 & 16.29 & -2.79 & 39.80 & 37.90 & -14.16 \\
\hline $10 \mathrm{~dB}$ & 24.57 & 24.10 & 6.85 & 35.52 & 15.98 & 32.41 & 26.68 & 6.86 & 26.34 & 13.73 & 14.03 & -0.07 \\
\hline $15 \mathrm{~dB}$ & 32.58 & 26.75 & 19.42 & 51.82 & 40.45 & 33.46 & 20.34 & 20.68 & 2.12 & 12.45 & 12.72 & 0.21 \\
\hline $\begin{array}{c}\text { RMS } \\
\text { Ave. }\end{array}$ & 21.05 & & & 32.24 & & & 21.56 & & & 21.33 & & \\
\hline
\end{tabular}

Table 5.5. Parameters of the two-distribution combinations that best fit the probability of occurrence for the attenuation thresholds

\begin{tabular}{|c|c|c|c|c|c|c|c|}
\hline \multirow{3}{*}{$A_{t h}$} & \multirow{3}{*}{$\alpha$} & \multicolumn{4}{|c|}{$P_{S}(D)$} & \multirow{2}{*}{\multicolumn{2}{|c|}{$\begin{array}{c}P_{L}(D) \\
\log \end{array}$}} \\
\hline & & \multicolumn{2}{|c|}{$\log$} & \multirow{2}{*}{$\begin{array}{l}\operatorname{Exp} \\
1 / E_{n} \\
\end{array}$} & \multirow{2}{*}{$\begin{array}{c}\text { Power } \\
B_{n} \\
\end{array}$} & & \\
\hline & & $\mu_{n, 1}$ & $\sigma_{n, 1}$ & & & $\mu_{n, 2}$ & $\sigma_{n, 2}$ \\
\hline \multicolumn{8}{|c|}{ Ka-band } \\
\hline $3 \mathrm{~dB}$ & 0.854 & -0.55 & 1.61 & 4.22 & 0.796 & 4.54 & 1.33 \\
\hline $5 \mathrm{~dB}$ & 0.821 & -0.30 & 1.56 & 2.27 & 0.800 & 4.91 & 1.29 \\
\hline $10 \mathrm{~dB}$ & 0.768 & -1.47 & 2.18 & 5.56 & 0.674 & 5.33 & 0.85 \\
\hline $15 \mathrm{~dB}$ & 0.760 & -4.02 & 3.00 & 8.18 & 0.667 & 5.16 & 1.48 \\
\hline \multicolumn{8}{|c|}{ Q-band } \\
\hline $3 \mathrm{~dB}$ & 0.835 & -9.05 & 3.19 & 5.44 & 1.031 & 4.16 & 1.62 \\
\hline $5 \mathrm{~dB}$ & 0.803 & -4.42 & 2.53 & 1.96 & 0.926 & 4.50 & 1.48 \\
\hline $10 \mathrm{~dB}$ & 0.747 & -7.10 & 3.37 & 7.52 & 0.816 & 4.69 & 1.31 \\
\hline $15 \mathrm{~dB}$ & 0.717 & -11.93 & 4.68 & 5.08 & 0.637 & 4.79 & 1.23 \\
\hline
\end{tabular}


Table 5.6. Parameters of the three-exponential distribution for the probability of occurrence

\begin{tabular}{|c|c|c|c|c|c|c|}
\hline \multirow[t]{2}{*}{$A_{t h}$} & \multicolumn{3}{|c|}{ Conditional probability of occurrence } & \multicolumn{3}{|c|}{ Characteristic durations } \\
\hline & $\alpha_{1}$ & $\alpha_{2}$ & $\alpha_{3}$ & $\beta_{1}$ & $\beta_{2}$ & $\beta_{3}$ \\
\hline \multicolumn{7}{|c|}{ Ka-band } \\
\hline $3 \mathrm{~dB}$ & 0.880 & 0.113 & 0.007 & 4.90 & 223.07 & 1256.0 \\
\hline $5 \mathrm{~dB}$ & 0.833 & 0.159 & 0.007 & 4.71 & 248.83 & 2181.4 \\
\hline $10 \mathrm{~dB}$ & 0.706 & 0.287 & 0.008 & 4.36 & 221.56 & 1096.6 \\
\hline $15 \mathrm{~dB}$ & 0.645 & 0.271 & 0.08 & 3.66 & 126.85 & 495.15 \\
\hline \multicolumn{7}{|c|}{ Q-band } \\
\hline $3 \mathrm{~dB}$ & 0.861 & 0.116 & 0.023 & 3.35 & 122.85 & 935.77 \\
\hline $5 \mathrm{~dB}$ & 0.792 & 0.164 & 0.044 & 3.51 & 96.50 & 751.46 \\
\hline $10 \mathrm{~dB}$ & 0.734 & 0.209 & 0.057 & 4.12 & 126.19 & 677.24 \\
\hline $15 \mathrm{~dB}$ & 0.752 & 0.235 & 0.013 & 5.52 & 223.96 & 1879.5 \\
\hline
\end{tabular}

In Table 5.5, $\alpha$ decreases while the threshold attenuation increases. Since it is related with the probability of occurrence of short events, its values represent that in all cases most of the events are short ones but their contributions gets lower as the threshold increases.

The $\alpha_{n}$ of Table 5.6 also reinforce the previous statements, although the modeling is different, since now the probability of occurrence is divided into three ranges instead of two. For the $\operatorname{Exp}+\operatorname{Exp}+\operatorname{Exp}$ fitting, the physical interpretation of $\beta_{n}$ values as the mean durations for the different kind of fades (short, medium and long) is also seen.

\subsubsection{Fading time modeling}

In the case of the fading time modeling, the three combinations of two distributions $(\log +\log$, Exp+Log, Pwr+Log) are employed again. Since the Log+Log combination gives the best overall results for the probability of occurrence, the procedure presented in $[\mathbf{2 2 3}]$ for deriving $F(D)$ from the $P(D)$ coefficients can be used. $T(D)$ is now obtained from $(2.25 \mathrm{~b})$ (see the Appendix 2 for the intermediate steps):

$$
T(D)=\mathrm{K}_{1} \cdot N_{\text {Tot }} \cdot e^{L_{1}} \cdot \operatorname{erfc}\left(\frac{\ln D-\mu_{n, 1}^{*}}{\sigma_{\mathrm{n}, 1} \sqrt{2}}\right)+\mathrm{K}_{2} \cdot N_{\text {Tot }} \cdot e^{L_{2}} \cdot \operatorname{erfc}\left(\frac{\ln D-\mu_{n, 2}^{*}}{\sigma_{\mathrm{n}, 2} \sqrt{2}}\right)
$$

with the following parameters, that are calculated from the $\alpha$ value and, the means and standard deviations of the $\log +\log$ distributions used to represent the probability of occurrence of fades in (2.22):

$$
\begin{gathered}
\mu_{n, 1}^{*}=\mu_{n, 1}+\sigma_{\mathrm{n}, 1}^{2} \\
\mu_{n, 2}^{*}=\mu_{n, 2}+\sigma_{\mathrm{n}, 2}^{2} \\
L_{1}==\mu_{n, 1}+\frac{\sigma_{\mathrm{n}, 1}^{2}}{2} \\
L_{2}==\mu_{n, 2}+\frac{\sigma_{\mathrm{n}, 2}^{2}}{2} \\
\mathrm{~K}_{1}=\frac{\alpha}{\operatorname{erfc}\left(\frac{\ln D_{0}-\mu_{\mathrm{n}, 1}}{\sigma_{\mathrm{n}, 1} \sqrt{2}}\right)}
\end{gathered}
$$




$$
\mathrm{K}_{2}=\frac{1-\alpha}{\operatorname{erfc}\left(\frac{\ln D_{0}-\mu_{\mathrm{n}, 2}}{\sigma_{\mathrm{n}, 2} \sqrt{2}}\right)}
$$

Then, the distribution for the total fraction of fading time, for durations starting at the minimum value $D_{0}$, is calculated as:

$$
F(D)=\frac{T(D)}{T\left(D_{0}\right)}
$$

The previous procedure is referred as UPM procedure in Table 5.7 and Figures 5.12 and 5.13. In these, the fittings and testing variables are shown for both experiments. The testing variable RMS, standard deviation and mean obtained by using in this case (5.3) and the obtained coefficients are collected in Tables 5.7 and 5.8 respectively.

\begin{tabular}{|c|c|c|c|c|c|c|c|c|c|c|c|c|}
\hline \multirow{3}{*}{$A_{t h}$} & \multicolumn{12}{|c|}{ Testing Variable for Fading Time (\%) } \\
\hline & \multicolumn{3}{|c|}{$\log +\log$} & \multicolumn{3}{|c|}{ Power $+\log$} & \multicolumn{3}{|c|}{$\operatorname{Exp}+\log$} & \multicolumn{3}{|c|}{ UPM procedure } \\
\hline & RMS & Std. & Mean & RMS & Std. & Mean & RMS & Std. & Mean & RMS & Std. & Mean \\
\hline \multicolumn{13}{|c|}{ Ka-band } \\
\hline $3 \mathrm{~dB}$ & 17.81 & 13.80 & -11.59 & 35.16 & 24.79 & -25.43 & 5.56 & 5.23 & 2.16 & 10.15 & 9.66 & 3.65 \\
\hline $5 \mathrm{~dB}$ & 23.13 & 16.89 & -16.17 & 37.62 & 26.57 & -27.19 & 5.54 & 5.26 & -2.06 & 9.31 & 9.28 & 2.02 \\
\hline $10 \mathrm{~dB}$ & 30.13 & 21.44 & -21.74 & 58.51 & 40.38 & -43.34 & 6.10 & 6.23 & 0.60 & 14.58 & 11.91 & 8.82 \\
\hline $15 \mathrm{~dB}$ & 35.83 & 21.85 & -28.96 & 60.80 & 36.47 & -49.54 & 20.97 & 15.28 & 14.90 & 16.74 & 12.01 & 12.05 \\
\hline $\begin{array}{l}\text { RMS } \\
\text { Ave. }\end{array}$ & 26.73 & & & 48.02 & & & 9.54 & & & 12.70 & & \\
\hline \multicolumn{13}{|c|}{ Q-band } \\
\hline $3 \mathrm{~dB}$ & 11.25 & 9.39 & -6.47 & 16.79 & 12.87 & -11.10 & 14.90 & 13.42 & 7.01 & 8.58 & 8.68 & 1.12 \\
\hline $5 \mathrm{~dB}$ & 17.24 & 13.65 & -10.89 & 21.26 & 16.21 & -14.14 & 15.44 & 13.78 & 7.49 & 9.35 & 9.40 & 1.59 \\
\hline $10 \mathrm{~dB}$ & 32.03 & 21.83 & -23.90 & 34.33 & 23.28 & -25.72 & 23.80 & 19.29 & 14.53 & 10.72 & 10.96 & -0.07 \\
\hline $15 \mathrm{~dB}$ & 20.83 & 14.07 & -15.66 & 22.88 & 15.16 & -17.45 & 12.58 & 10.62 & -7.13 & 35.89 & 9.69 & -34.62 \\
\hline $\begin{array}{l}\text { RMS } \\
\text { Ave. }\end{array}$ & 20.34 & & & 23.81 & & & 16.68 & & & 16.13 & & \\
\hline
\end{tabular}

Table 5.7. Testing variable for the total fraction of fading time curves

\begin{tabular}{|c|c|c|c|c|c|c|c|}
\hline \multirow{3}{*}{$A_{t h}$} & \multirow{3}{*}{$\alpha$} & \multicolumn{4}{|c|}{$F_{S}(D)$} & \multirow{2}{*}{\multicolumn{2}{|c|}{$\begin{array}{c}F_{L}(D) \\
\log \end{array}$}} \\
\hline & & \multicolumn{2}{|c|}{$\log$} & \multirow{2}{*}{$\begin{array}{l}\operatorname{Exp} \\
1 / E_{t}\end{array}$} & \multirow{2}{*}{$\begin{array}{c}\text { Power } \\
B_{t}\end{array}$} & & \\
\hline & & $\mu_{t, 1}$ & $\sigma_{t, 1}$ & & & $\mu_{t, 2}$ & $\sigma_{t, 2}$ \\
\hline \multicolumn{8}{|c|}{ Ka-band } \\
\hline $3 \mathrm{~dB}$ & 0.110 & 2.62 & 2.66 & 11.39 & 0.1298 & 6.28 & 1.28 \\
\hline $5 \mathrm{~dB}$ & 0.060 & 2.46 & 2.80 & 10.44 & 0.1382 & 6.58 & 1.34 \\
\hline $10 \mathrm{~dB}$ & 0.058 & 3.38 & 2.61 & 16.23 & 0.0889 & 6.13 & 0.87 \\
\hline $15 \mathrm{~dB}$ & 0.094 & 4.65 & 3.77 & 22.83 & 0.0532 & 5.77 & 0.72 \\
\hline \multicolumn{8}{|c|}{ Q-band } \\
\hline $3 \mathrm{~dB}$ & 0.107 & 0.44 & 4.97 & 13.53 & 0.1634 & 6.65 & 1.45 \\
\hline $5 \mathrm{~dB}$ & 0.095 & -0.11 & 7.04 & 18.15 & 0.1218 & 6.59 & 1.36 \\
\hline $10 \mathrm{~dB}$ & 0.138 & 0.29 & 15.44 & 40.0 & 0.0524 & 6.30 & 1.15 \\
\hline $15 \mathrm{~dB}$ & 0.021 & -1.51 & 4.54 & 6.10 & 0.2365 & 6.47 & 1.58 \\
\hline
\end{tabular}

Table 5.8. Fitting parameters for the total fraction of fading time distributions 

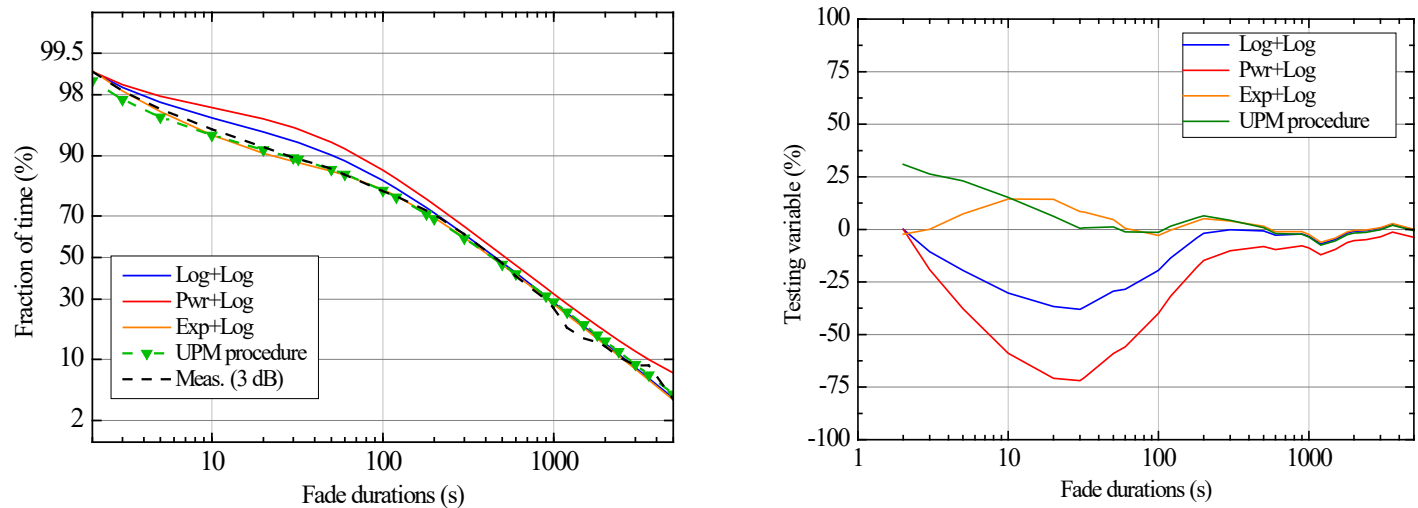

a)
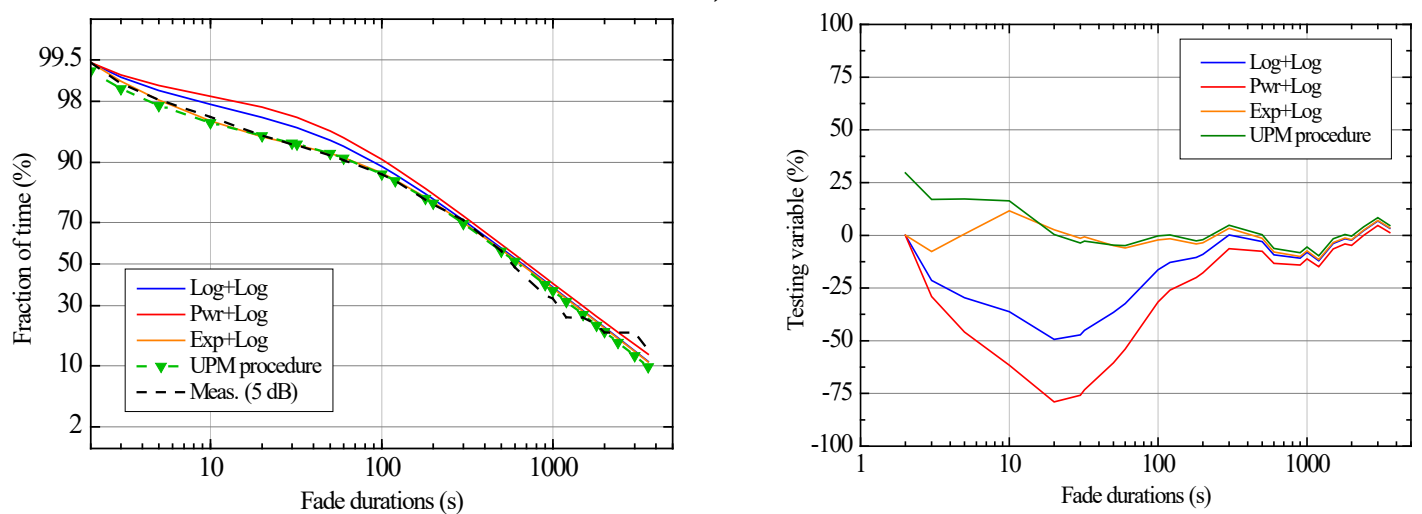

b)
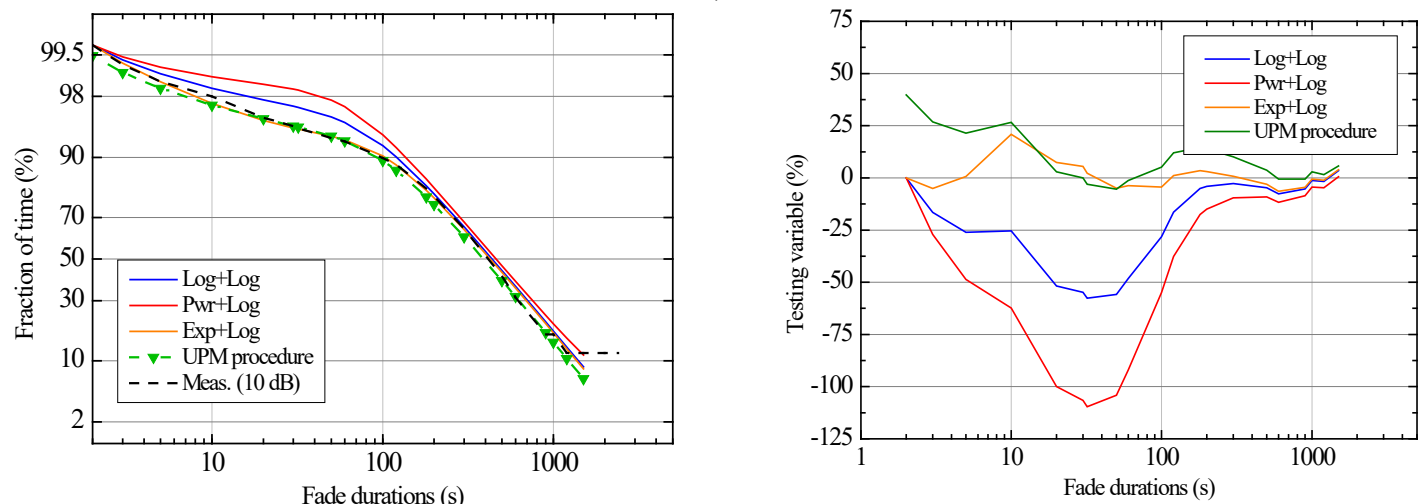

c)
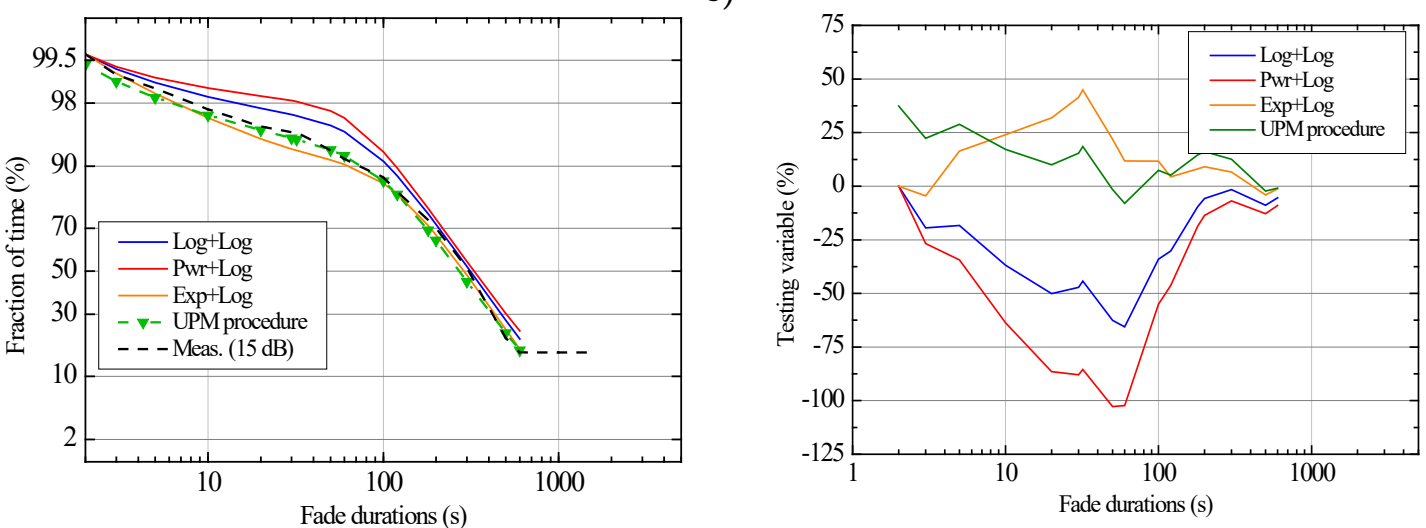

d)

Figure 5.12. Ka-band fading time fitting results (left-hand column) and testing variable (right-hand column) for $A_{\text {th }}$ equal to a) $3 \mathrm{~dB}$, b) $5 \mathrm{~dB}$, c) $10 \mathrm{~dB}$ and d) $15 \mathrm{~dB}$. 

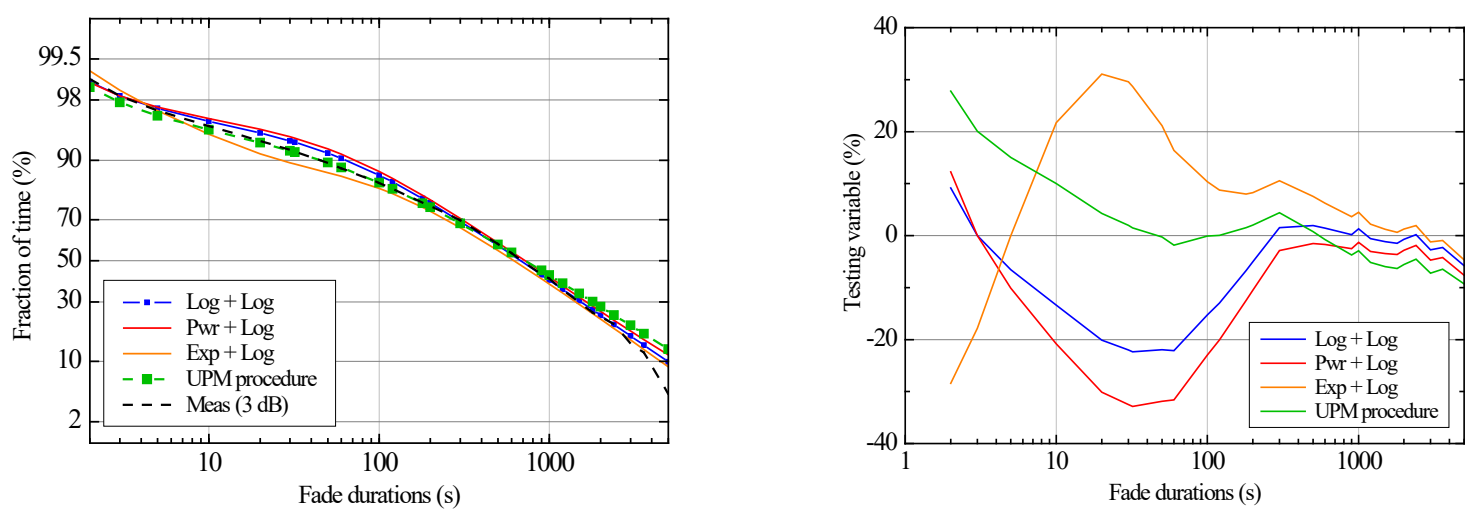

a)
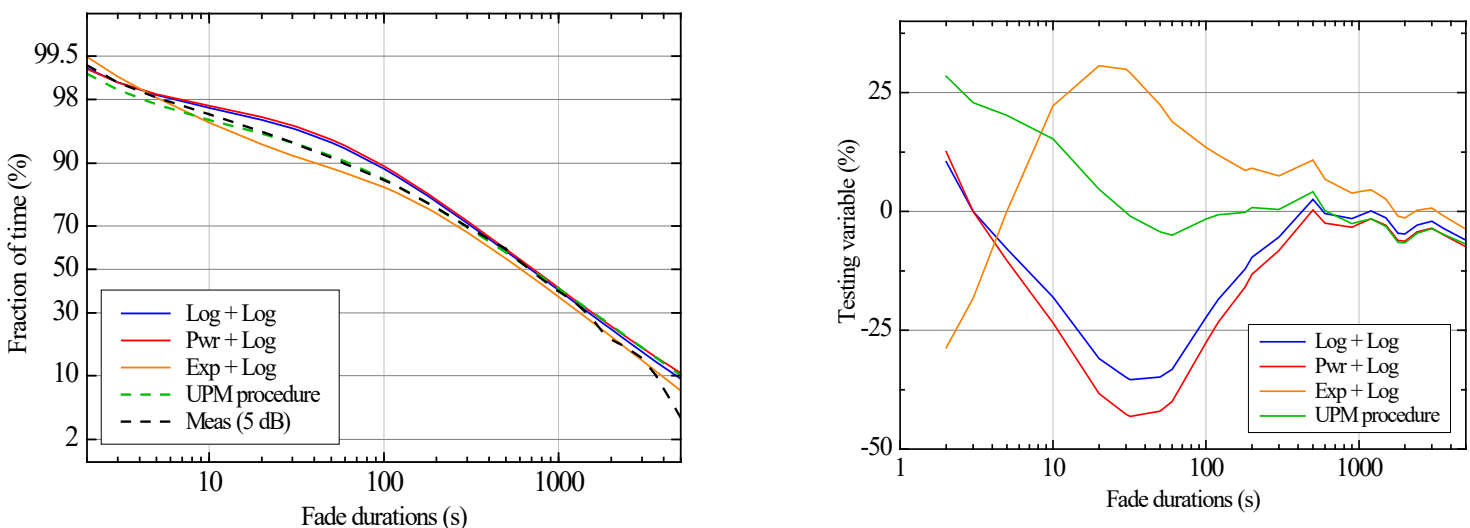

b)
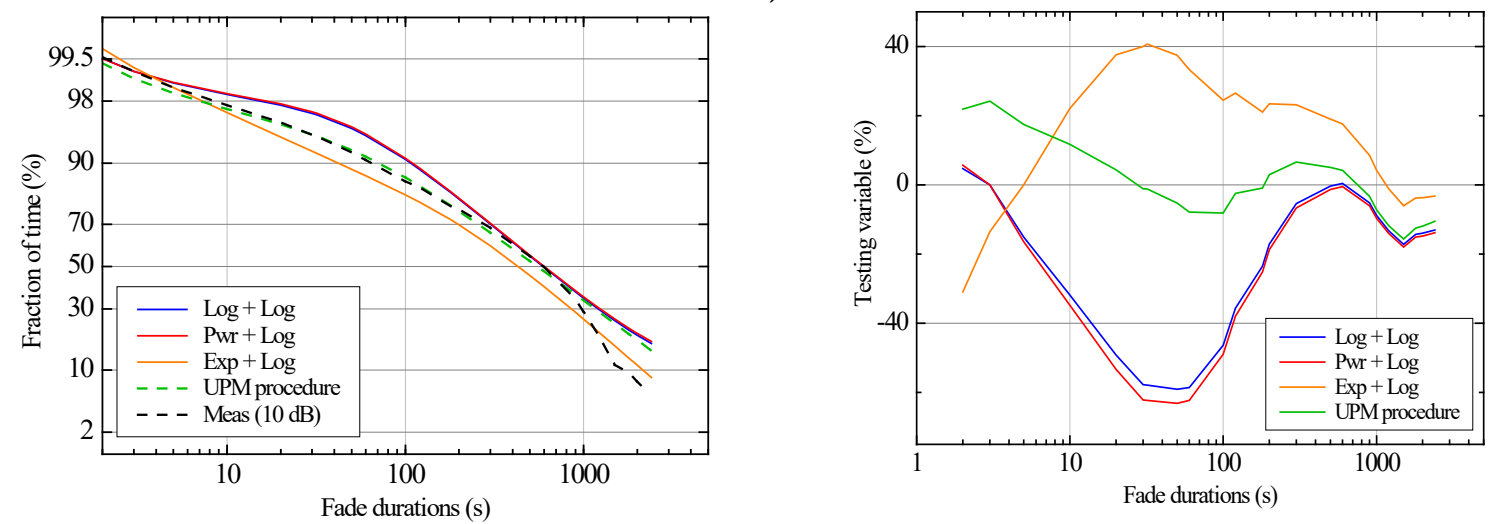

c)
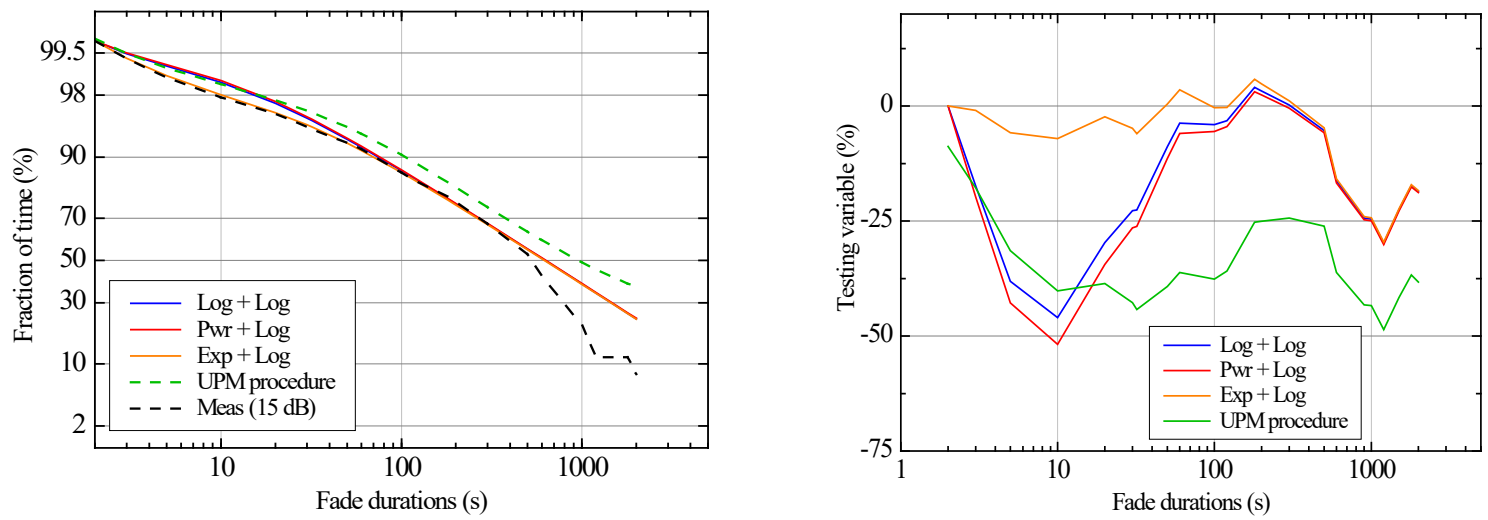

d)

Figure 5.13. Q-band fading time fitting results (left-hand column) and testing variable (right-hand column) for $A_{\text {th }}$ equal to a) $3 \mathrm{~dB}$, b) $5 \mathrm{~dB}, \mathrm{c}$ ) $\left.10 \mathrm{~dB}, \mathrm{~d}\right) 15 \mathrm{~dB}$. 
From this fitting, the UPM procedure gives very good approximations for both cases, comparable with the values obtained with the Exp+Log fitting (the overall best fit combination). The advantage of this procedure is that it relies on a physical basis (the total fading time depends on the total number of fade events) and can be used directly from the two-term log-normal parameters obtained for the probability of occurrence distributions.

\subsubsection{Inter-fade duration results}

In the case of inter-fade duration (IFD), there is not an accepted model to compare with it. Similar to the case of fade durations, the inter-fade duration distribution results are shown in Figures 5.14 and 5.15 for the Ka- and Q-bands respectively for certain attenuation thresholds $A_{\text {th }}$ of $3,5,10$ and $15 \mathrm{~dB}$.

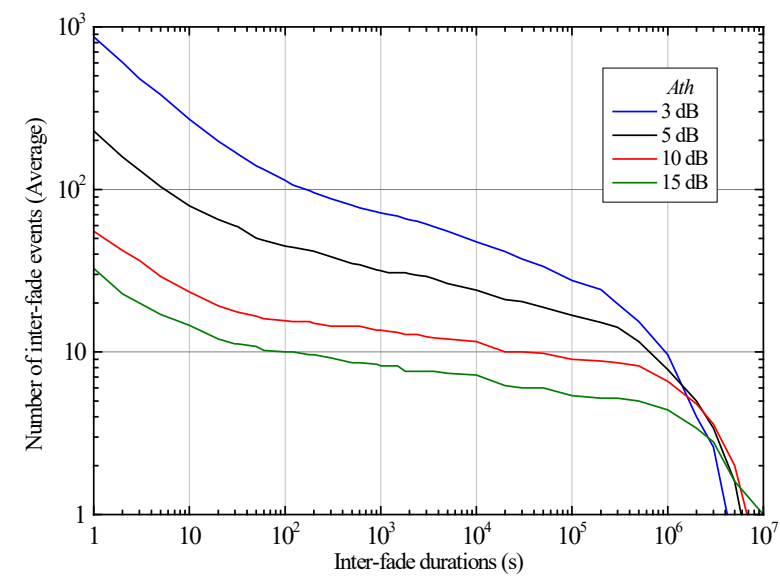

a)

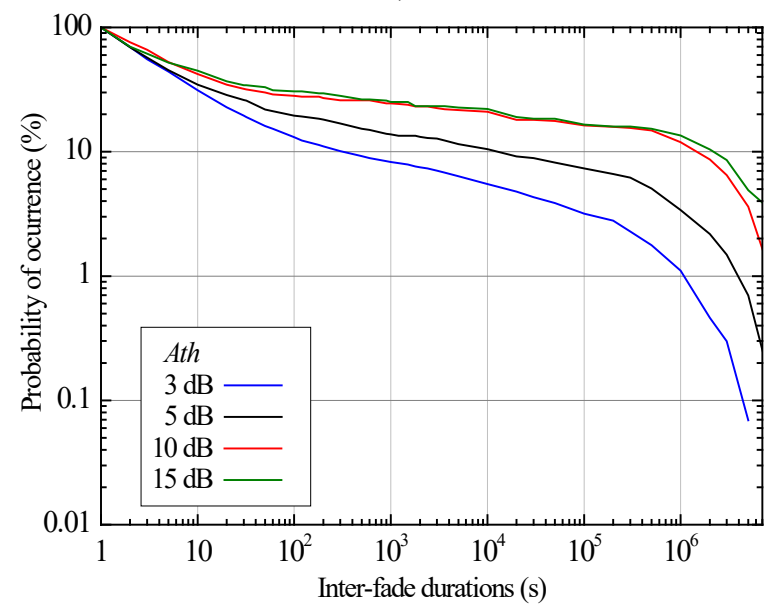

c)

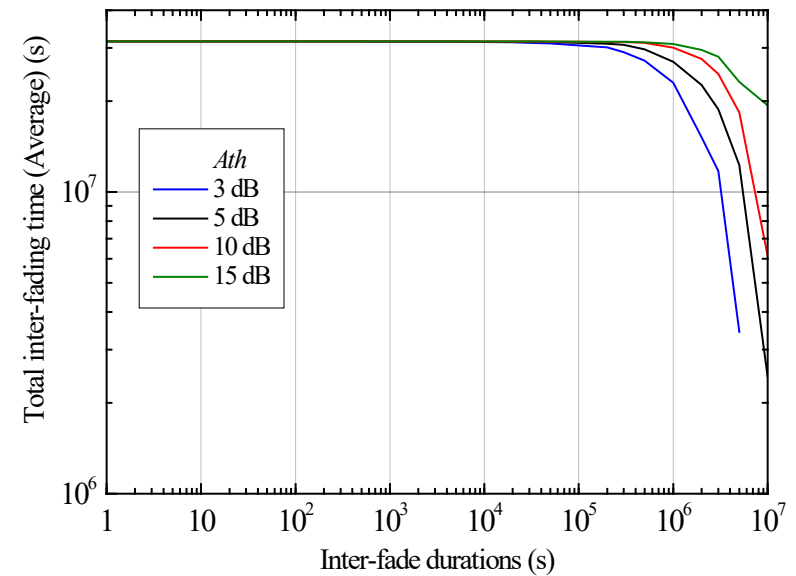

b)

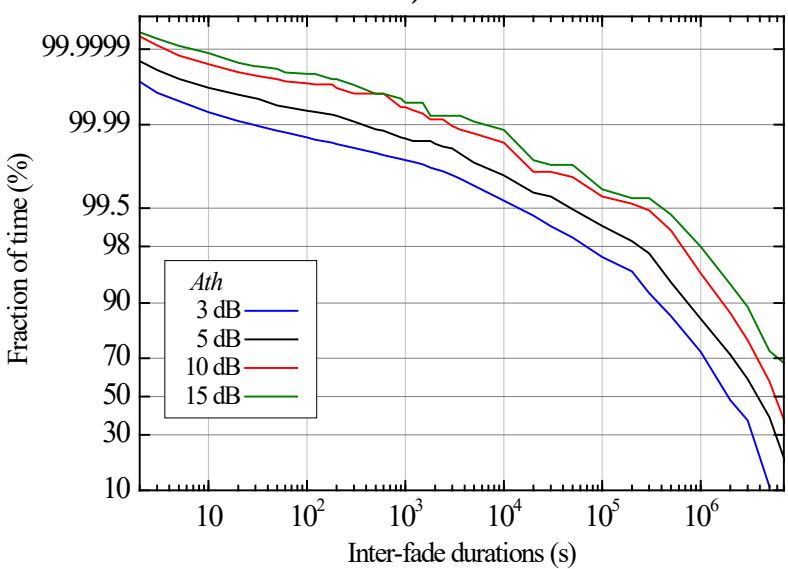

d)

Figure 5.14. IFD results for the Ka-band, a) number of events (Ave.), b) total interfading time (Ave.), c) probability of occurrence and d) fraction of time. 


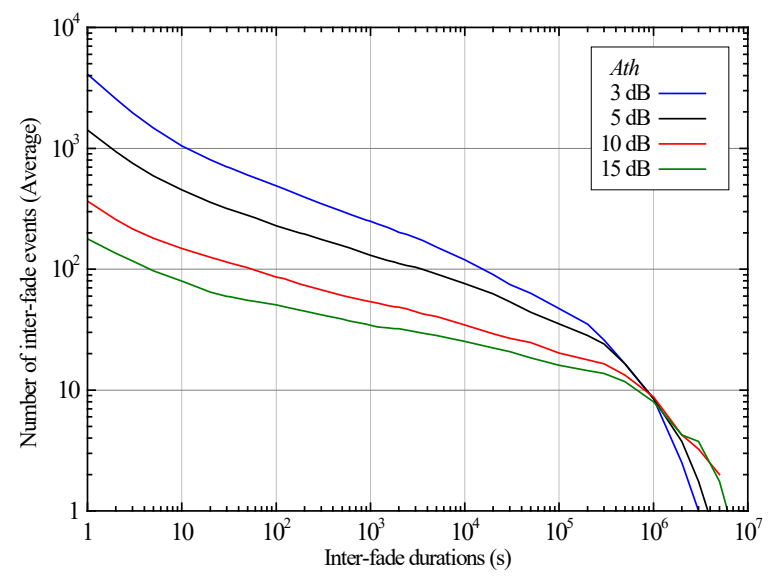

a)

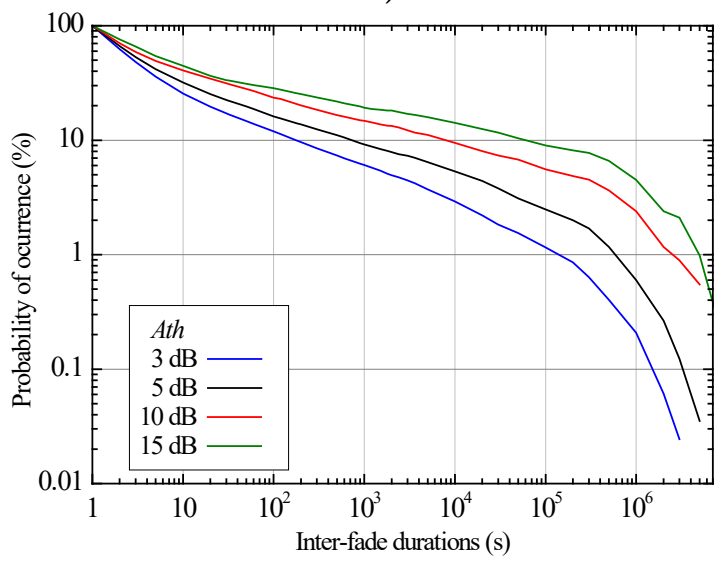

c)

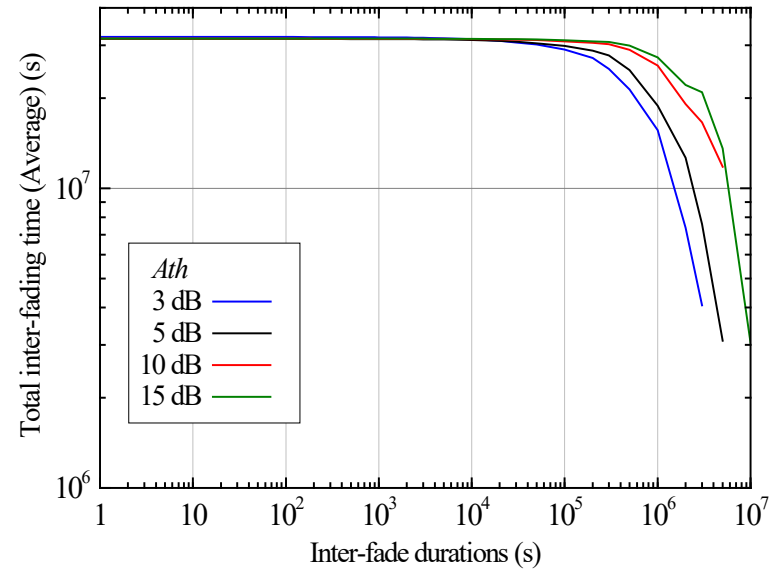

b)

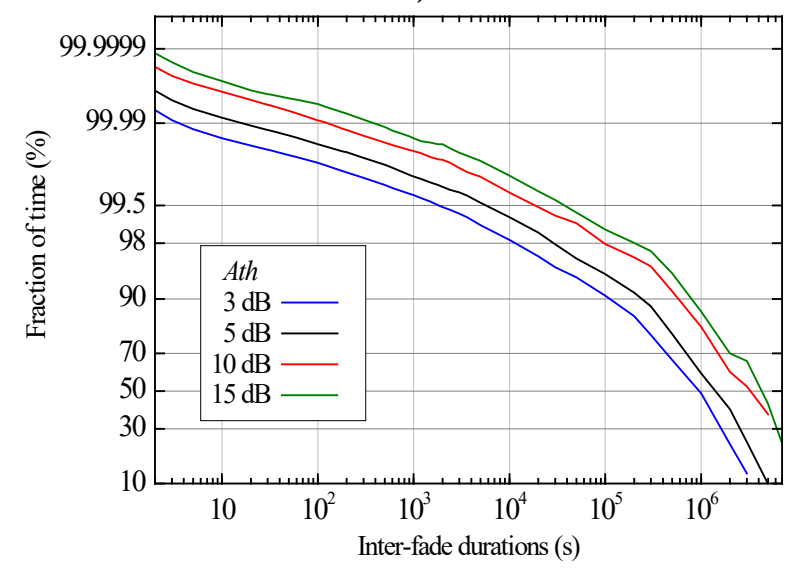

d)

Figure 5.15. IFD results for the Q-band, a) number of events (Ave.), b) total inter-fading time (Ave.), c) probability of occurrence and d) fraction of time.

The only available model (i.e. the three-lognormal terms USA model, which was presented in sub-section 2.5.2) does not give adequate results with the UPM data. Maybe the use of data only from the ACTS campaign is the reason behind this behavior. Also, it can be questioned whether the use of three combined distributions is or is not enough to describe accurately the inter-fade duration data, or, on the other hand, if it is possible to use a twodistribution combination for obtaining a predicted inter-fade duration distribution with good precision. In order to contribute in finding an answer to these questions, a modeling effort has been carried out with the available data.

\subsubsection{IFD probability of occurrence modeling}

Keeping in mind the procedure proposed by ONERA in [112], some modifications initially outlined in [224] were applied for modeling the inter-fade probability of occurrence:

- Using a value in the range of 900 to $1800 \mathrm{~s}$ as a limit between the first (related with short durations) and the second distribution (related with longer durations). This is analogous to the $D_{\min }$ value for the FD case.

- Setting up an upper limit of $10^{6} \mathrm{~s}$ to the inter-fade duration (longer durations would not be modeled). 
With these considerations the inter-fade duration distributions can be modeled with very good precision by two combined log-normal distributions (the other combinations did not produce relevant results). The graphical results are displayed in Figure 5.16 for the probability of occurrence modeling as well as the behavior of the testing variable of (5.2).
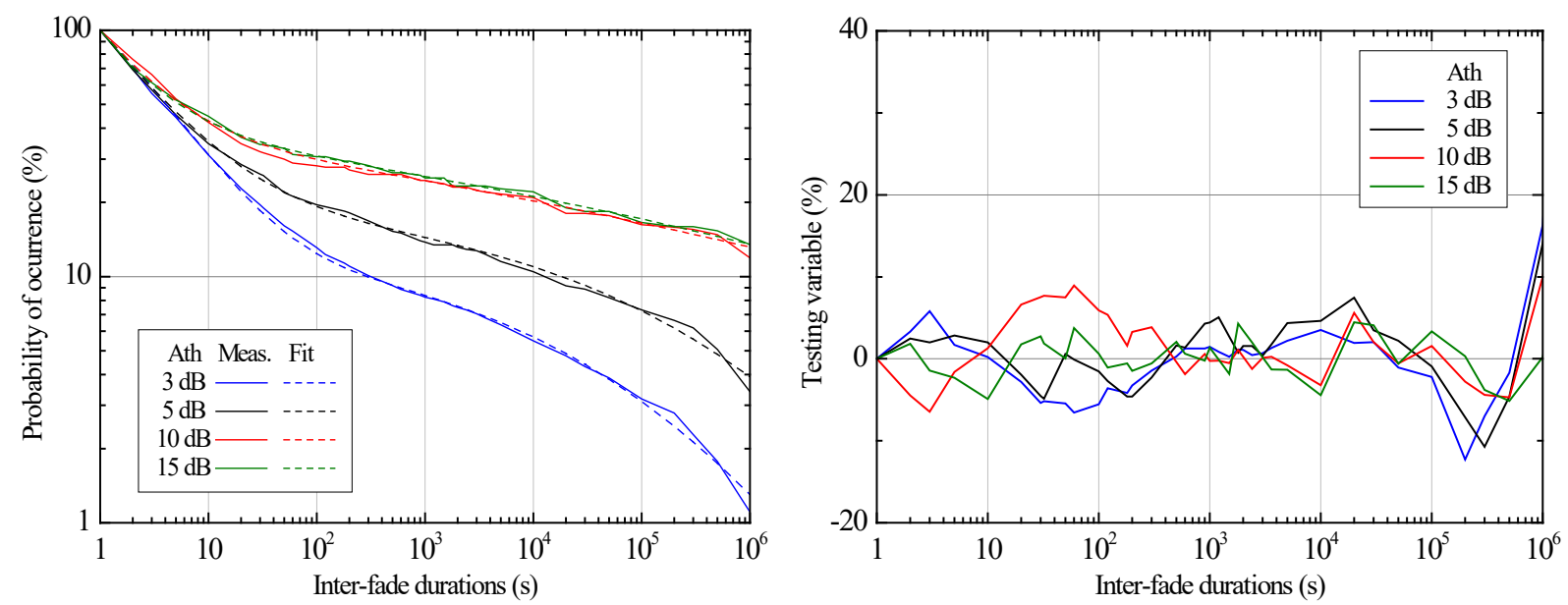

a)
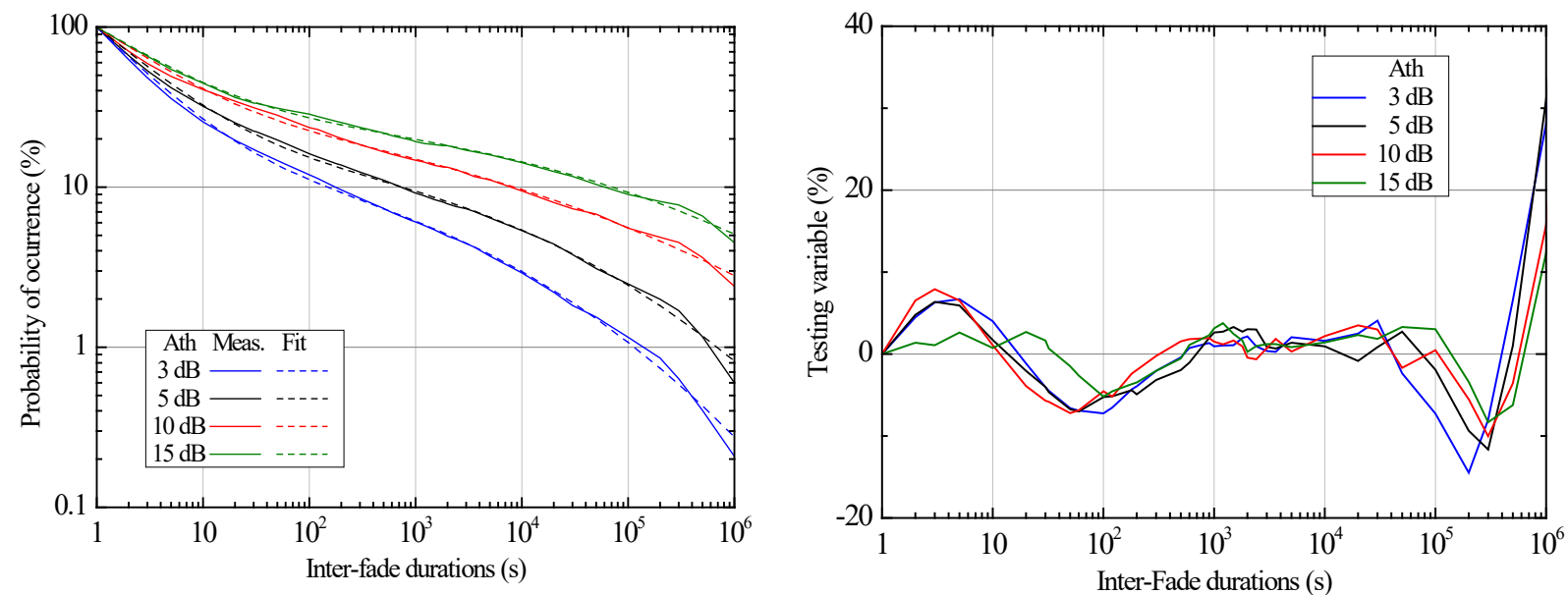

b)

Figure 5.16. Two-term log-normal fitting procedure resulting graphs (left-hand column) and testing variable behavior (right-hand column) of IFD probability of occurrence for a) Ka- and b) Q-bands.

The obtained parameters (similar to those obtained in the case of FD for the two lognormal distribution) are given in Table 5.9. In these, $R M S$ ( $U p$ to $10^{6} s$ ) is the RMS value obtained by using the testing variable of (5.2) up to the limit of $10^{6} \mathrm{~s}$, whereas $R M S$ (Full) stands for the RMS values obtained following the same procedure but including all the experimental results without a limit.

The modeling works fine up to the imposed limit, and the errors are higher when durations above $10^{6} \mathrm{~s}$ are taken into account. Here, the $\alpha$ values have the same behavior as in the FD case. The parameters that characterize the short and long IFD distributions have values in the same order of magnitude, although for the $15 \mathrm{~dB} \mu_{n, 1}$ in the Ka-band case has a rather odd one. 
Table 5.9. IFD two-term log-normal distribution parameters for the experiments and RMS (in \%) values of the testing variable

\begin{tabular}{c|ccccc|cc}
\hline \hline$A_{t h}(\mathrm{~dB})$ & $\alpha$ & $\mu_{n, 1}$ & $\sigma_{n, 1}$ & $\mu_{n, 2}$ & $\sigma_{n, 2}$ & RMS (Up to 10 $\left.{ }^{6} \mathrm{~s}\right)$ & RMS (Full) \\
\hline \multicolumn{7}{c}{ Ka-band } \\
\hline 3 & 0.888 & -1.80 & 2.57 & 9.22 & 3.83 & 4.61 & 39.63 \\
5 & 0.836 & -19.12 & 5.83 & 10.89 & 4.09 & 4.45 & 51.40 \\
10 & 0.620 & -11.80 & 4.15 & 1.27 & 14.02 & 4.28 & 47.03 \\
15 & 0.619 & -172.2 & 14.30 & 5.33 & 11.96 & 2.50 & 56.59 \\
\hline \multicolumn{7}{c}{ Q-band } \\
\hline 3 & 0.878 & -13.14 & 4.54 & 6.62 & 3.57 & 6.66 & 32.07 \\
5 & 0.854 & -12.89 & 4.83 & 7.87 & 3.72 & 6.70 & 42.58 \\
10 & 0.720 & -5.07 & 3.45 & 6.32 & 5.52 & 4.80 & 23.92 \\
15 & 0.736 & -8.25 & 4.38 & 9.56 & 4.81 & 3.55 & 49.41 \\
\hline \hline
\end{tabular}

A second analysis was oriented to test if a meaningful improvement can be obtained by using a three log-normal function combination for fitting the complete probability of occurrence distribution, especially for modeling the IFD longer than $10^{6} \mathrm{~s}$. This three lognormal function is described as:

$$
P(D)=\alpha_{1} \cdot \frac{\operatorname{erfc}\left(\frac{\ln D-\mu_{n, 1}}{\sigma_{n, 1} \sqrt{2}}\right)}{\operatorname{erfc}\left(\frac{\ln D_{0}-\mu_{n, 1}}{\sigma_{n, 1} \sqrt{2}}\right)}+\alpha_{2} \cdot \frac{\operatorname{erfc}\left(\frac{\ln D-\mu_{n, 2}}{\sigma_{n, 2} \sqrt{2}}\right)}{\operatorname{erfc}\left(\frac{\ln D_{0}-\mu_{n, 2}}{\sigma_{n, 2} \sqrt{2}}\right)}+\alpha_{3} \cdot \frac{\operatorname{erfc}\left(\frac{\ln D-\mu_{n, 3}}{\sigma_{n, 3} \sqrt{2}}\right)}{\operatorname{erfc}\left(\frac{\ln D_{0}-\mu_{n, 3}}{\sigma_{n, 3} \sqrt{2}}\right)}
$$

where the meaning of $\alpha_{i}$ is analogous to the previous case, i.e. each $\alpha_{i}$ represents the fraction of the probability of occurrence of each associated log-normal distribution (in the FD case $\alpha_{2}$ can be seen as $\left.1-\alpha_{1}\right)$. In (5.12), two new parameters are introduced: $\mu_{n, 3}$ and $\sigma_{n, 3}$ being the mean and standard deviation of the third log-normal function. In this case, all the parameters are optimized at once, using the interior-point algorithm [225] with the constraint that $\sum \alpha_{i}=1$ and with no specific limit between the different distribution ranges. The results are presented in Figure 5.17 and Table 5.10.

Table 5.10. IFD fitting parameters for the three log-normal function combination and testing variable RMS values (in \%)

\begin{tabular}{|c|c|c|c|c|c|c|c|c|c|c|c|}
\hline $\begin{array}{c}A_{t h} \\
(\mathrm{~dB}) \\
\end{array}$ & $\alpha_{1}$ & $\mu_{n, 1}$ & $\sigma_{n, 1}$ & $\alpha_{2}$ & $\mu_{n, 2}$ & $\sigma_{n, 2}$ & $\alpha_{3}$ & $\mu_{n, 3}$ & $\sigma_{n, 3}$ & $\begin{array}{c}\text { RMS } \\
\left.\text { (Up to } 10^{6} \mathrm{~s}\right)\end{array}$ & $\begin{array}{l}\text { RMS } \\
\text { (Full) } \\
\end{array}$ \\
\hline \multicolumn{12}{|c|}{ Ka-band } \\
\hline 3 & 0.924 & -27.63 & 7.16 & 0.051 & 9.49 & 1.93 & 0.025 & 13.55 & 1.03 & 1.26 & 5.82 \\
\hline 5 & 0.791 & -73.36 & 10.38 & 0.171 & 7.44 & 4.91 & 0.037 & 13.73 & 0.89 & 2.01 & 34.19 \\
\hline 10 & 0.790 & -2274 & 65.46 & 0.208 & 13.92 & 1.50 & 0.001 & -964.9 & 625.2 & 7.74 & 14.51 \\
\hline 15 & 0.641 & -3681 & 97.22 & 0.197 & 14.38 & 1.59 & 0.162 & 0.69 & $4 \mathrm{e}-10$ & 6.50 & 8.03 \\
\hline \multicolumn{12}{|c|}{ Q-band } \\
\hline 3 & 0.860 & -155.09 & 13.71 & 0.131 & 5.95 & 2.92 & 0.010 & 12.68 & 1.03 & 3.67 & 4.58 \\
\hline 5 & 0.791 & -209.72 & 16.46 & 0.189 & 5.60 & 3.47 & 0.020 & 12.86 & 1.11 & 3.68 & 11.37 \\
\hline 10 & 0.523 & -681.94 & 26.57 & 0.443 & 0.12 & 6.05 & 0.034 & 13.50 & 0.94 & 2.59 & 2.82 \\
\hline 15 & 0.775 & -44.20 & 9.30 & 0.156 & 8.64 & 2.66 & 0.069 & 14.20 & 0.96 & 2.60 & 8.16 \\
\hline
\end{tabular}



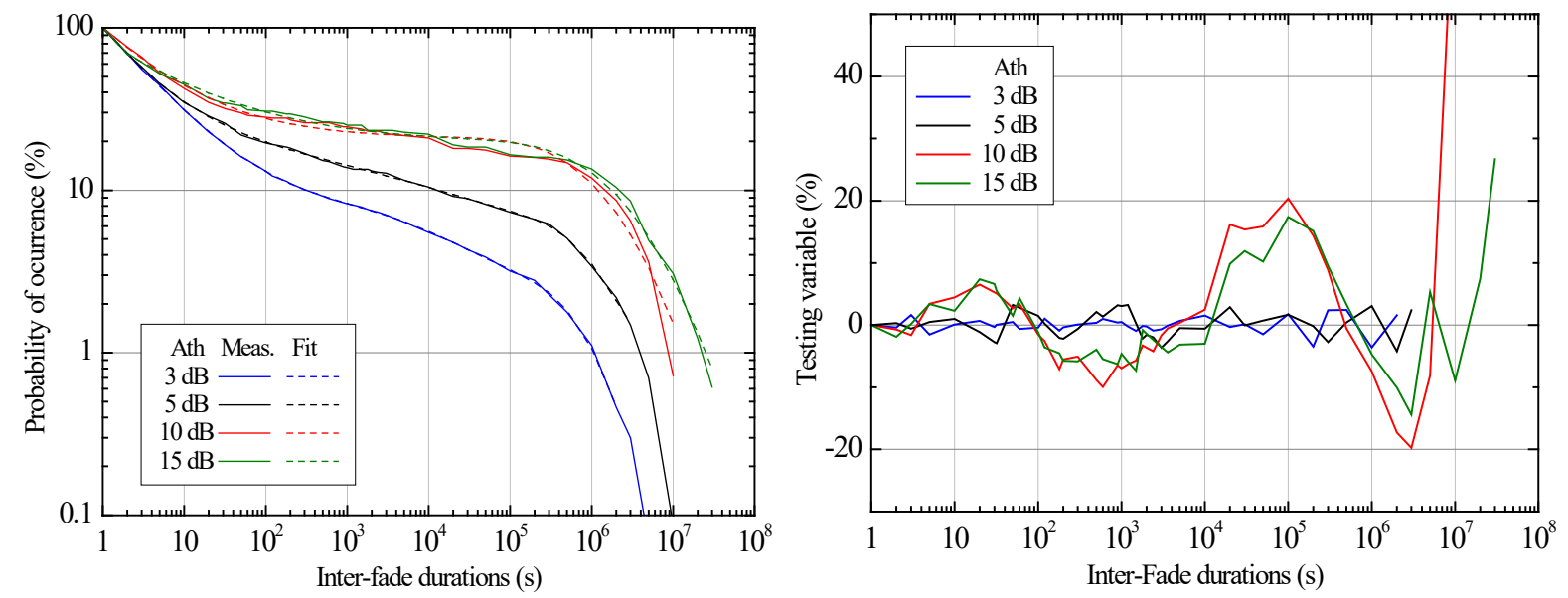

a)
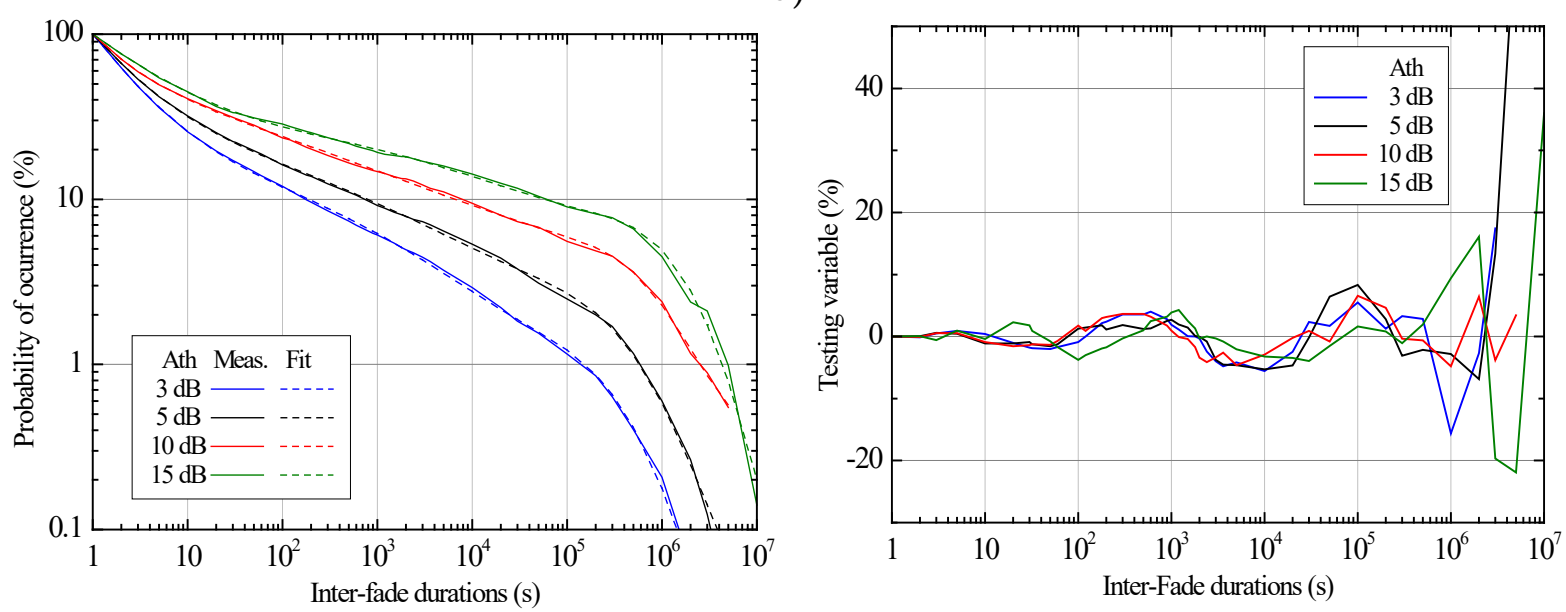

b)

Figure 5.17. Three log-normal function fitting procedure resulting graphs (left-hand column) and testing variable behavior (right-hand column) for the IFD probability of occurrence for a) Ka- and b) Q-bands.

From the comparison of Tables 5.9 and 5.10, an improvement can be seen regarding to the RMS value of the testing variable that takes into account the durations higher than $10^{6} \mathrm{~s}$ $(R M S(F u l l))$. The RMS obtained for durations up to this limit do not show remarkable improvements compared with those obtained when the two-term log-normal fitting (with constraints) was used, casting some doubts on the use of an additional log-normal term. It seems only useful for modeling such long durations (longer than $10^{6} \mathrm{~s}$ ) that have little practical relevance, since they occur very few times in an average year. Nevertheless, the use of a third distribution seems to be absolutely necessary if the modeling of such long durations is intended.

Preliminary results on the Ka- and Q-band IFD distributions, either for the two- or the three-term log-normal combination, have been published in [224].

\subsubsection{Inter-fading time modeling}

When the two-distribution combination fittings were used for the inter-fading time modeling, using the described fitting procedure, the results largely deviate from the 
experimental data in most of the cases. Only the two log-normal function distribution gives a good approximation. The parameters of this fitting are given in Table 5.11 and the graphical comparisons are shown in Figure 5.18.

Table 5.11. Fitting parameters for the total fraction of inter-fading time distributions with two log-normal function distributions

\begin{tabular}{|c|c|c|c|c|c|c|c|}
\hline \multirow[t]{2}{*}{$A_{t h}$} & \multirow[t]{2}{*}{$\alpha$} & \multicolumn{2}{|c|}{$\begin{array}{c}F_{S}(D) \\
\log \end{array}$} & \multicolumn{2}{|c|}{$\begin{array}{c}F_{L}(D) \\
\log \end{array}$} & \multicolumn{2}{|c|}{ Testing Variable for inter-fading Time (\%) } \\
\hline & & $\mu_{t, 1}$ & $\sigma_{t, 1}$ & $\mu_{t, 2}$ & $\sigma_{t, 2}$ & RMS (Up to $10^{6} \mathrm{~s}$ ) & RMS full \\
\hline \multicolumn{8}{|c|}{ Ka-band } \\
\hline $3 \mathrm{~dB}$ & 0.0117 & 16.44 & 5.86 & 17.02 & 2.63 & 10.30 & 22.79 \\
\hline $5 \mathrm{~dB}$ & 0.0049 & 17.51 & 5.58 & 17.98 & 2.81 & 11.63 & 34.35 \\
\hline $10 \mathrm{~dB}$ & 0.0025 & 28.01 & 8.71 & 21.05 & 3.42 & 19.49 & 56.91 \\
\hline $15 \mathrm{~dB}$ & 0.0001 & 21.96 & 7.40 & 22.41 & 3.70 & 19.20 & 72.46 \\
\hline \multicolumn{8}{|c|}{ Q-band } \\
\hline $3 \mathrm{~dB}$ & 0.0213 & 14.70 & 5.74 & 14.95 & 2.72 & 8.98 & 14.52 \\
\hline $5 \mathrm{~dB}$ & 0.0163 & 15.53 & 5.28 & 15.90 & 2.76 & 9.82 & 21.01 \\
\hline $10 \mathrm{~dB}$ & 0.0061 & 15.30 & 4.83 & 17.78 & 3.07 & 11.26 & 27.48 \\
\hline $15 \mathrm{~dB}$ & 0.0039 & 15.70 & 4.78 & 18.14 & 2.95 & 9.76 & 31.83 \\
\hline
\end{tabular}

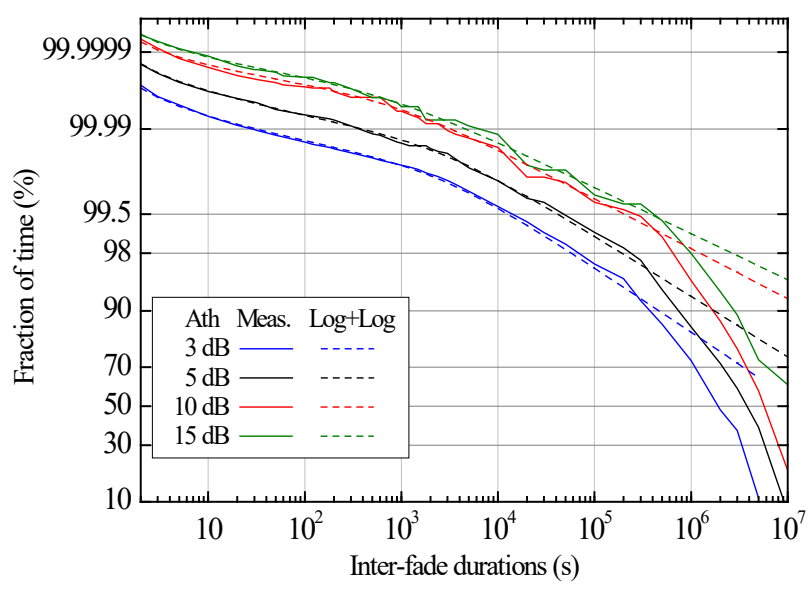

a)

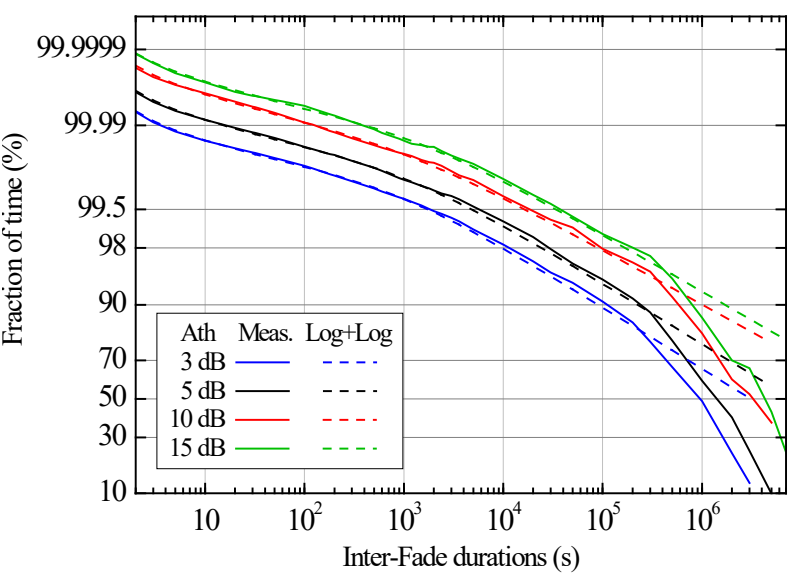

b)

Figure 5.18. Two log-normal function combination fitting procedure resulting graphs of inter-fading time for a) the Ka- and b) Q-bands.

From Table 5.11, a stable behavior can be seen in almost all log-normal parameters. Now, $\alpha$ has a very low value in all cases, with a decreasing trend when the attenuation threshold increases. The use of a three log-normal function combination does not provide meaningful results: it gives an approximation with no physical meaning and in some cases the longer durations are not well fitted.

\subsubsection{Fade slope results and modeling}

The fade slope statistics were obtained by applying a 5th-order Butterworth filter with a cutoff frequency of $0.02 \mathrm{~Hz}$ to the time series of excess attenuation. For comparing with the 
ITU-R fade slope model, the values of $b=2.3$ and $s=0.01$ were used, as recommended for Europe. The cutoff frequency of (2.42) is selected at $0.025 \mathrm{~Hz}$, as the ITU-R recommends.

In the case of fade slope, a testing method for comparing the fade slope predictions with measurements is provided in $[\mathbf{2 2 1}]$. In it, the CCDF of fade slope exceeded at a given attenuation threshold is used. For each threshold $A$ and fade slope value $\zeta$, the test variable $\varepsilon$ is calculated from the predicted exceedance probability $P_{p}(\zeta \mid A)$ and the measured exceedance probability $P_{m}(\zeta \mid A)$ :

$$
\varepsilon(\zeta, A)=2 \cdot \frac{P_{p}(\zeta \mid A)-P_{m}(\zeta \mid A)}{P_{p}(\zeta \mid A)+P_{m}(\zeta \mid A)}
$$

The fade slope probability of occurrence and cumulative distributions for time intervals $\Delta t$ of 2 and $30 \mathrm{~s}$ are represented in Figures 5.19 and 5.20 for the Ka- and Q-bands respectively.
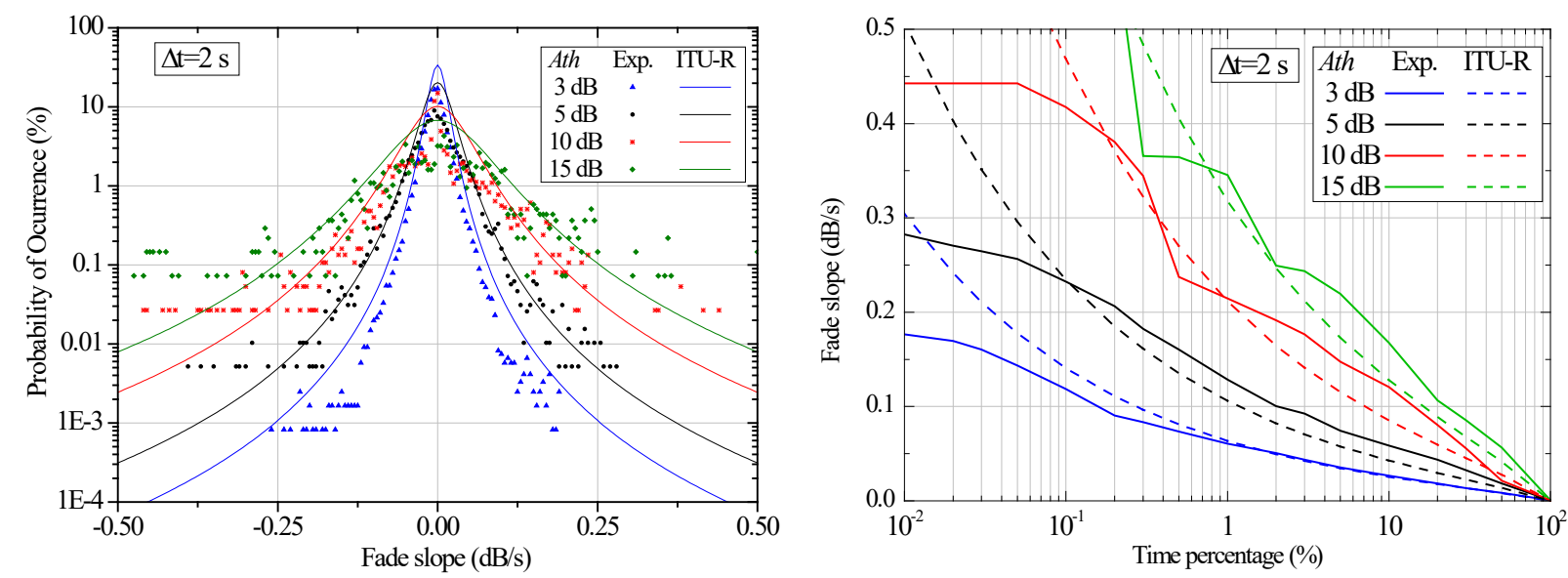

a)
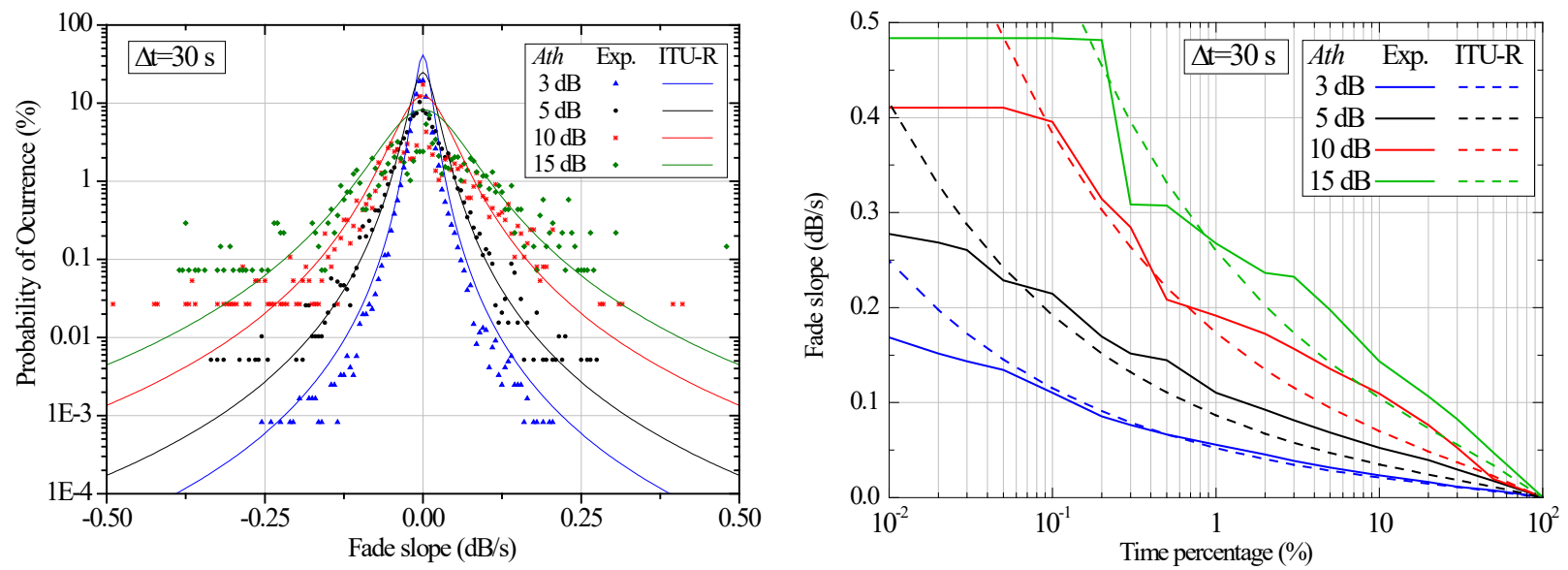

b)

Figure 5.19. Ka-band fade slope results compared with the Rec. ITU-R P.1623-1 model for a $\Delta t$ of a) $2 \mathrm{~s}$ and b) $30 \mathrm{~s}$ (In the left-hand the probability of occurrence and in the right-hand the cumulative distribution). 

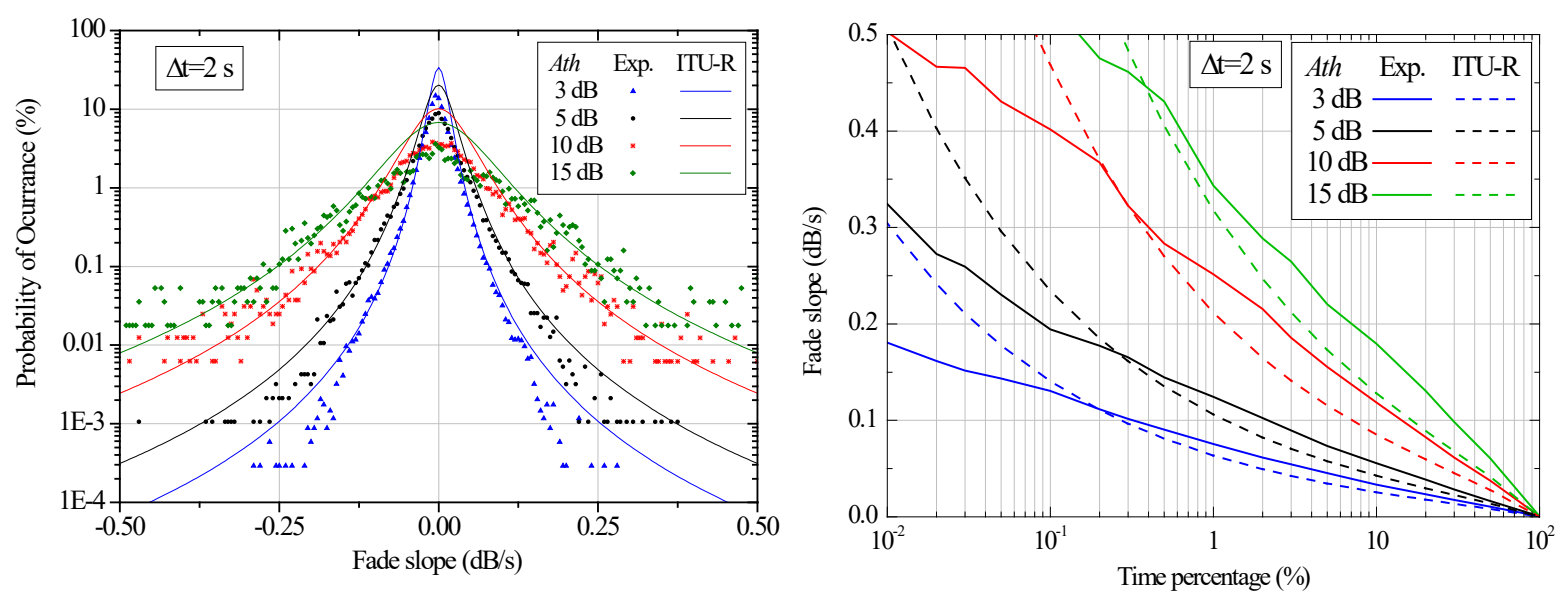

a)
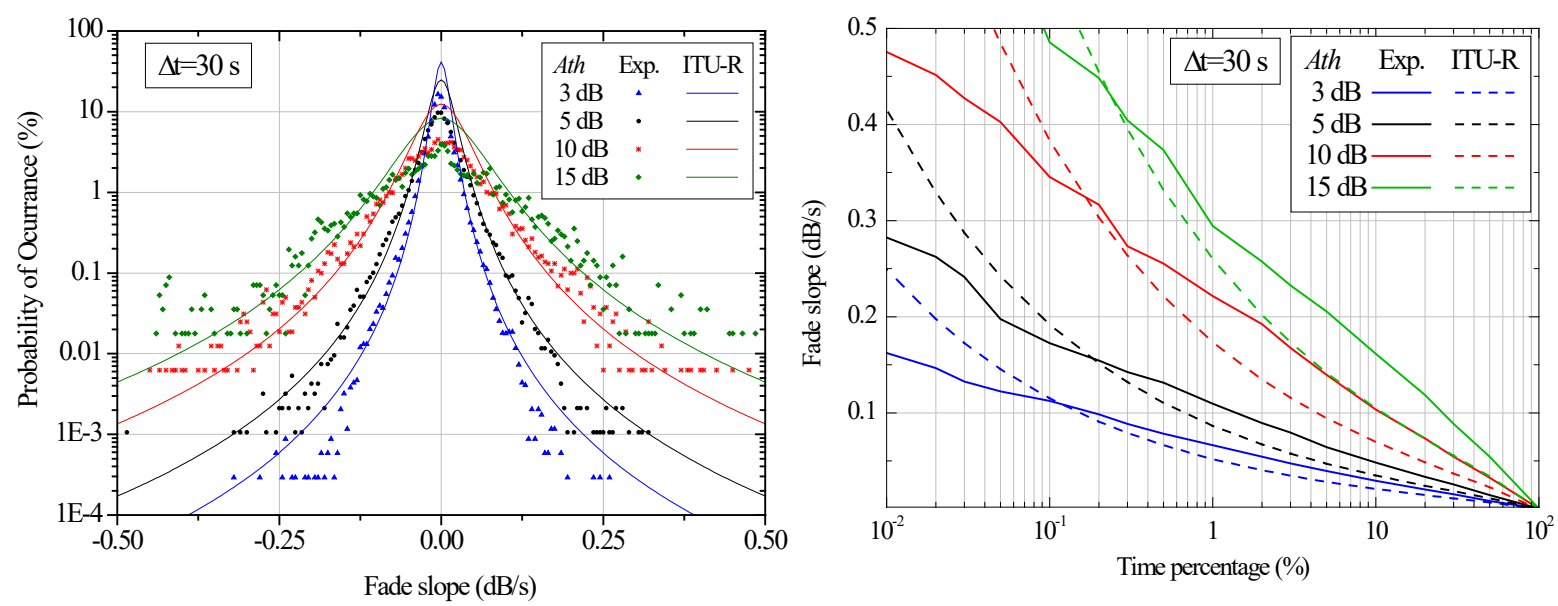

b)

Figure 5.20. Q-band fade slope results compared with the Rec. ITU-R P.1623-1 model for a $\Delta t$ of a) $2 \mathrm{~s}$ and b) $30 \mathrm{~s}$ (In the left-hand the probability of occurrence and in the righthand the cumulative distributions).

Table 5.12 shows the RMS values (in \%) of the testing variable of (5.13) for the cumulative distribution of fade slope for intervals between 2 and $180 \mathrm{~s}$, for probabilities from $0.01 \%$ to $50 \%$. The results show similar behaviors for the given intervals. Overall, the model accuracy deteriorates for higher $\Delta t$ and higher attenuation thresholds $A_{t h}$.

Table 5.12. RMS values (in \%) for the cumulative distributions for several $\Delta t$ and $A_{t h}$

\begin{tabular}{c|cc|cc|cccc}
\hline \hline \multirow{2}{*}{$\boldsymbol{\Delta}$} & \multicolumn{2}{|c|}{$\mathbf{3} \mathbf{d B}$} & \multicolumn{2}{c|}{$\mathbf{5 ~ d B}$} & \multicolumn{2}{c}{ 10 dB } & \multicolumn{2}{c}{$\mathbf{1 5} \mathbf{d B}$} \\
& Q-band & Ka-band & Q-band & Ka-band & Q-band & Ka-band & Q-band & Ka-and \\
\hline $\mathbf{2}$ & 40.24 & 43.11 & 32.01 & 42.69 & 25.54 & 27.92 & 20.01 & 15.79 \\
$\mathbf{1 0}$ & 39.89 & 42.19 & 32.28 & 42.82 & 26.12 & 28.36 & 20.02 & 16.63 \\
$\mathbf{3 0}$ & 38.60 & 33.73 & 35.60 & 42.69 & 29.89 & 32.85 & 24.40 & 27.63 \\
$\mathbf{6 0}$ & 33.15 & 22.07 & 39.31 & 45.07 & 36.89 & 47.71 & 44.09 & 50.50 \\
$\mathbf{1 2 0}$ & 32.06 & 35.23 & 40.90 & 50.22 & 54.31 & 58.31 & 60.75 & 57.59 \\
$\mathbf{1 8 0}$ & 37.51 & 29.66 & 47.72 & 62.88 & 59.21 & 61.76 & 72.64 & 67.82 \\
\hline \hline
\end{tabular}




\subsection{Time diversity results}

Time diversity results for the 4-year concurrent period of both experiments and certain time delays conveniently chosen (i.e. $60 \mathrm{~s}, 3 \mathrm{~min}, 5 \mathrm{~min}, 10 \mathrm{~min}, 30 \mathrm{~min}$ and $1 \mathrm{~h}$ ) are shown in Figure 5.21. The decision of presenting the 4-year concurrent period instead of the 5-year Ka-band period is based on comparison purposes. The selected delays have into account their meaningfulness. Then, delays longer than 1 hour may not have relevance on practical systems and, since rain events usually last for more than 1 minute, shorter delays are not considered. It is convenient to notice that the curve of $\delta t=0 \mathrm{~s}$ corresponds to the exceeded attenuation distribution (no diversity). Also, the statistical independency distribution (Stat. Indep. curve in the figures) is given for the probabilities $p$ :

$$
p_{\text {Stat.Indep. }}(\%)=\left(\frac{p}{100}\right)^{2} \cdot 100 \%
$$

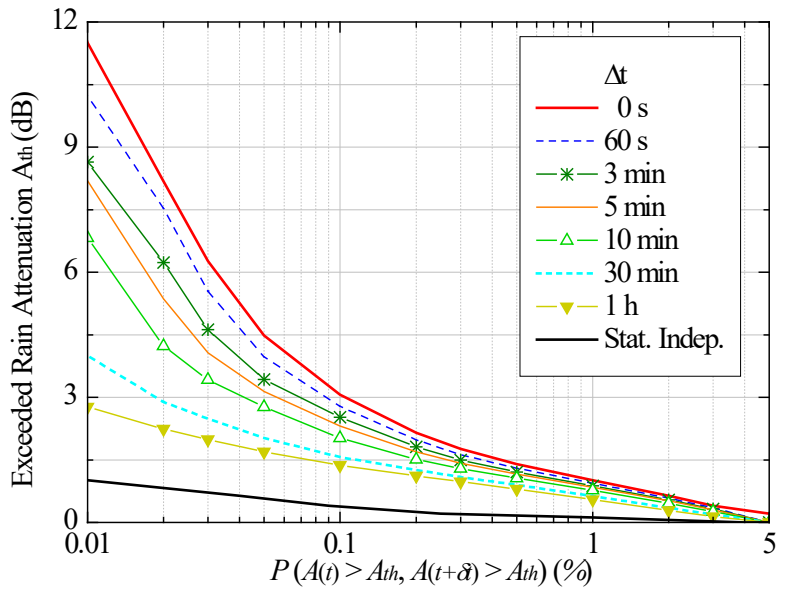

a)

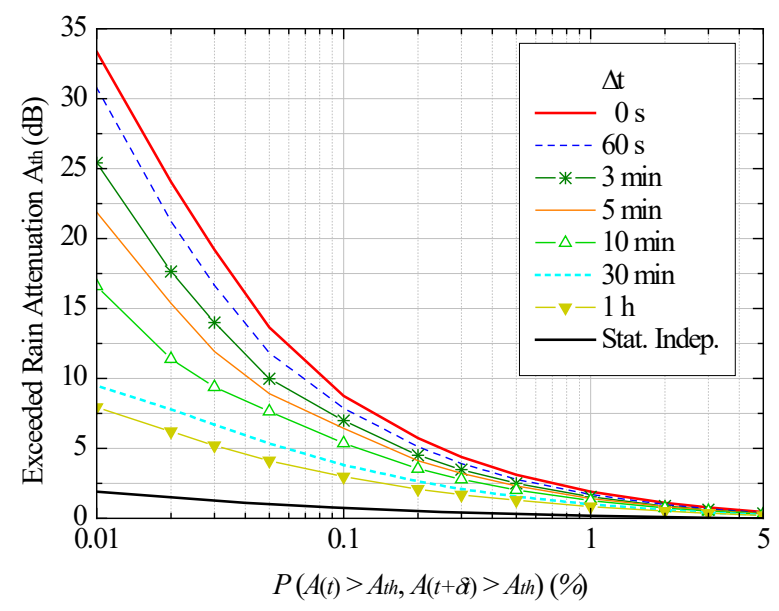

b)

Figure 5.21. Time Diversity results. Probability of exceedance for a) Ka- and b) Q-band.

The analyzed outage probability interval is restricted from $0.01 \%$ to $3 \%$, as was done in [226], since the data has better statistical stability for probabilities of more than $0.01 \%$, and the probability of rain in Madrid for an average year is usually less than $3 \%$ (however, some figures are limited to $5 \%$ for illustrative purposes).

From the experimental distributions, the diversity gain for a given $p$ and $\delta t$ can be calculated with (2.49), having the results of Figure 5.22 for both experiments. 


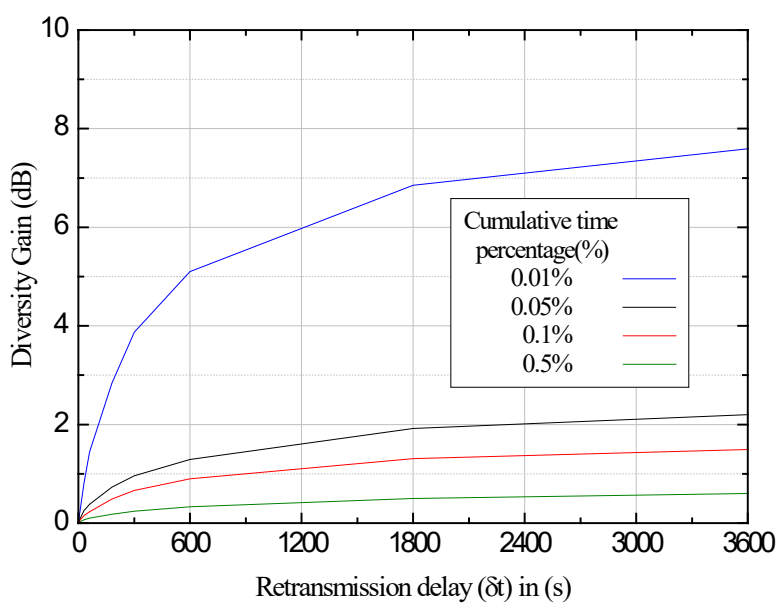

a)

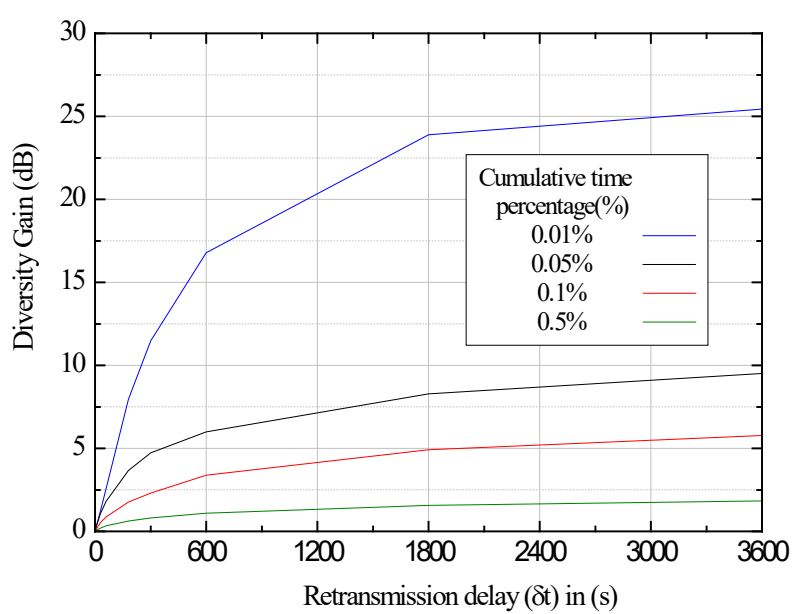

b)

Figure 5.22. Time Diversity results. Diversity gain for a) Ka- and b) Q-band.

In both cases, the gain rapidly increases for time delays of up to 10 minutes $(600 \mathrm{~s})$, specially for lower probabilities, with smaller increments for higher delays. For probabilities higher than $0.5 \%$ (corresponding to the lower attenuation values), the diversity gain is very low. Comparing the diversity gain of both experiments, higher values can be achieved for the Q-band measurements, for which higher attenuation levels are also reached.

\subsubsection{Comparison with models}

From the previous results, a comparison with the four TD models presented in Chapter 2 has been carried out, since they are not yet widely validated ones. When comparing their performances, it must be noted that the Joint Probability model needs parameters that are not available for Madrid in the references, so they have been obtained from the measurements. Consequently, in this case it is used more as an analysis model than as a prediction one and for this reason it is treated first. Here, the order of addressing the models has been changed regarding sub-section 2.6.1, in order to relate common parameters between procedures.

\section{Joint Probability model}

Using the definitions of the Joint Probability model, $m_{0}=m_{\delta t}$ and $\sigma_{0}=\sigma_{\delta t}$ (hence $\left.X_{0}=X_{\delta t}\right)$. These parameters can be estimated with the procedure of Recommendation ITUR P. 1057-4 [140]. The other parameters $\alpha, \tau_{1}$ and $\tau_{2}$ are obtained by regression of (2.54), as Figure 5.23 shows. All the model parameters are collected in Table 5.13 for both frequency bands.

Table 5.13. Parameters estimated for the Joint Probability Model

\begin{tabular}{lccccccc}
\hline \hline Satellite & $m_{0}$ & $\sigma_{0}$ & $P_{\text {down }}(\%)$ & $P_{u p}(\%)$ & $\alpha$ & $\tau_{1} \cdot 10^{4}$ & $\tau_{2} \cdot 10^{6}$ \\
\hline Ka-band & -4.33 & 1.80 & 0.01 & 3 & 0.57 & 4.35 & 3.17 \\
Q-band & -4.15 & 2.06 & 0.01 & 3 & 0.62 & 3.21 & 6.13 \\
\hline \hline
\end{tabular}




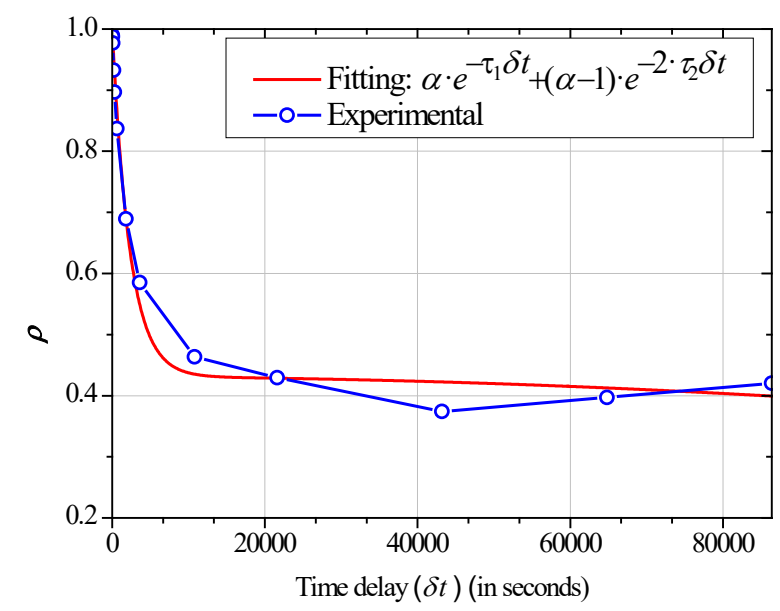

a)

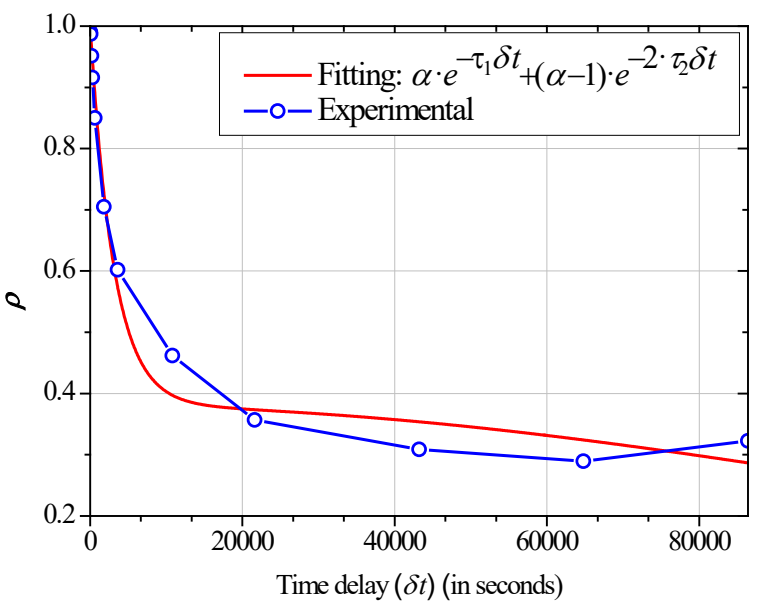

b)

Figure 5.23. Graphical regression of equation (2.54) for obtaining $\alpha, \tau_{1}$ and $\tau_{2}$ of the Joint Probability model for the a) Ka- and b) Q-band experiments.

\section{Greece model}

In the case of the Greece model, the mean $\left(m_{0}\right)$ and standard deviation $\left(\sigma_{0}\right)$ used are the same as appear in Table 5.13, using now a $\beta$ value of $2.687 \cdot 10^{-4} \mathrm{~s}^{-1}$ for the Ka-band and of $1.255 \cdot 10^{-4} s^{-1}$ for the Q-band. The parameter $\beta$ is derived with the procedure and software developed in an ESA study [227].

\section{ONERA model}

The parameters needed for the ONERA model are collected in Table 5.14. It is necessary to remember that in this case the mean $(m)$, the standard deviation $(\sigma)$ and $\beta$ are obtained for the rain distribution normalized to the probability of occurrence of rain for an average year using the same procedures as for the Greece model.

Table 5.14. Parameters estimated for the ONERA Model

\begin{tabular}{|c|c|c|c|c|}
\hline Satellite & $m$ & $\sigma$ & $P_{0}(\%)$ (estimated) & $\beta\left(10^{-4} s^{-1}\right)$ \\
\hline Ka-band & -1.52 & 1.39 & \multirow{2}{*}{3.990} & 4.52 \\
\hline Q-band & -0.73 & 1.51 & & 2.34 \\
\hline
\end{tabular}

\section{Matricciani TD model}

The Matricciani TD model, on the other hand, is quite straightforward with no need of relevant intermediate parameters.

\section{Comparisons}

Graphical comparisons between experimental and model results are presented in Figures 5.24 and 5.25, for certain time delays. 


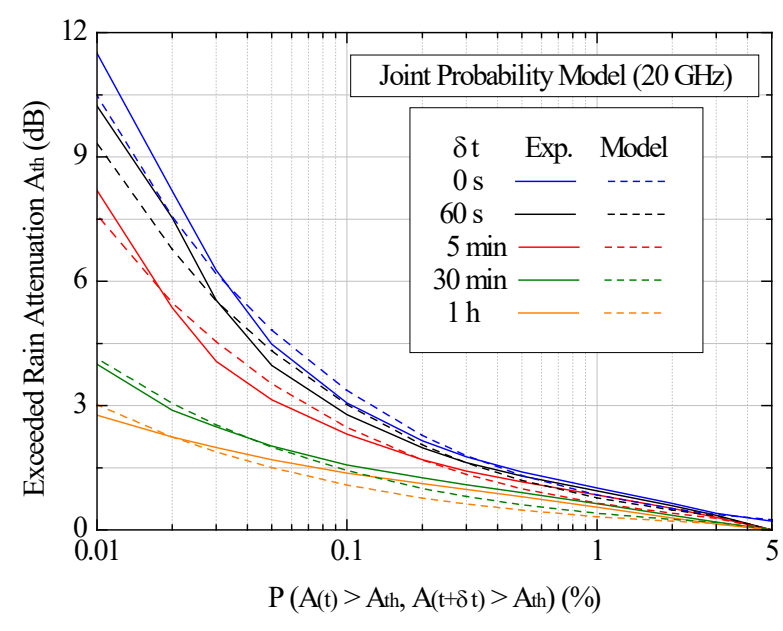

a)

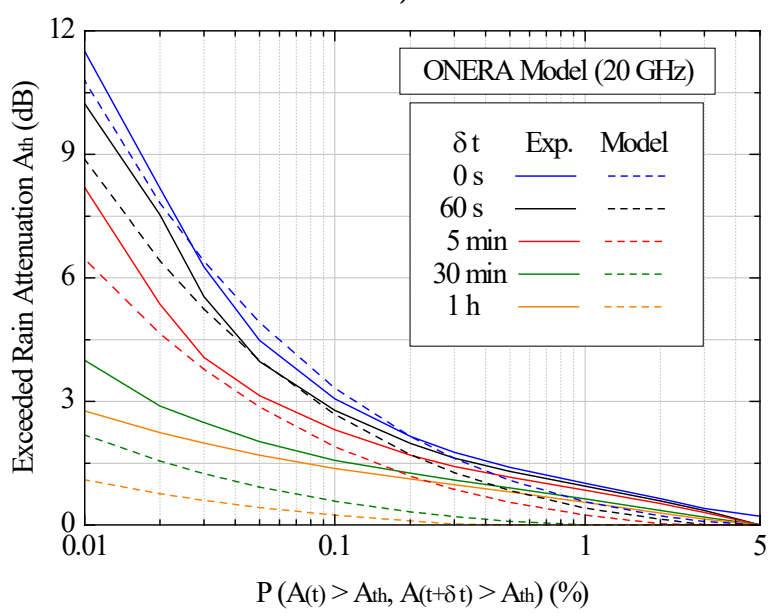

c)

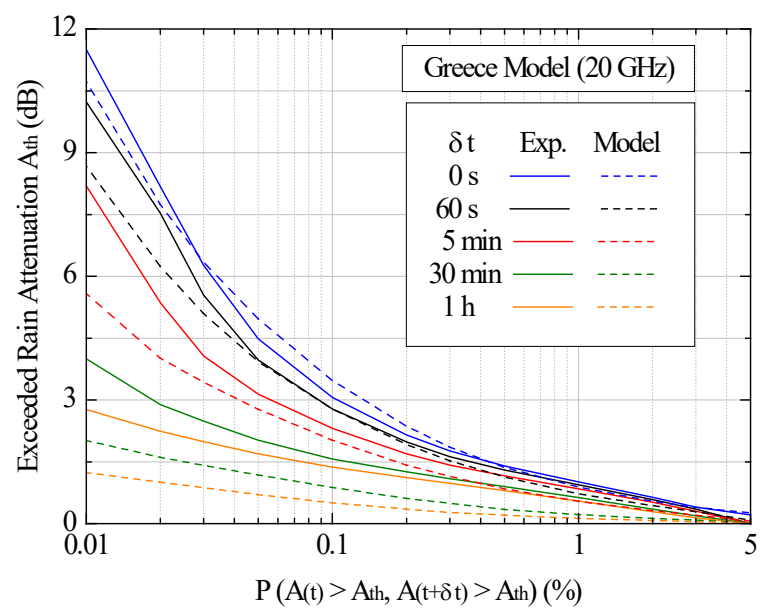

b)

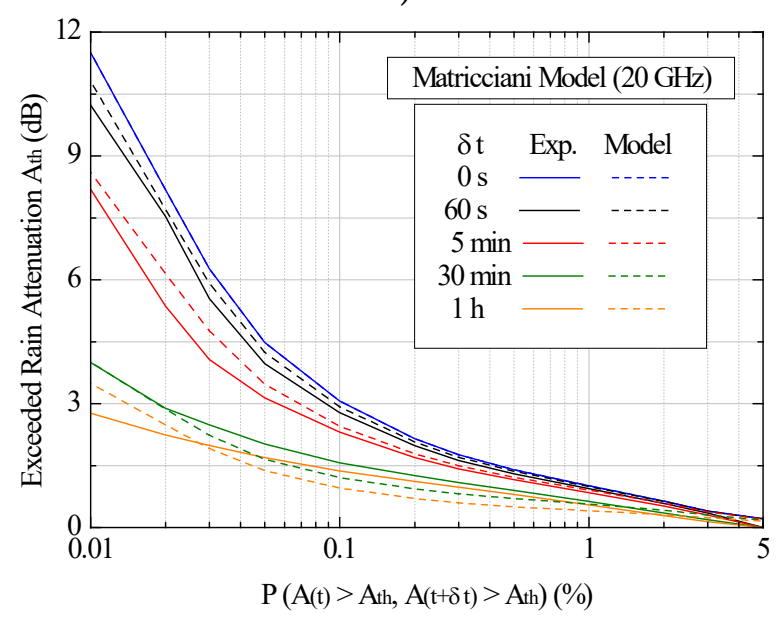

d)

Figure 5.24. TD models compared individually with the Ka-band results for certain $\delta t$, a) Joint Probability, b) Greece, c) ONERA and d) Matricciani. 


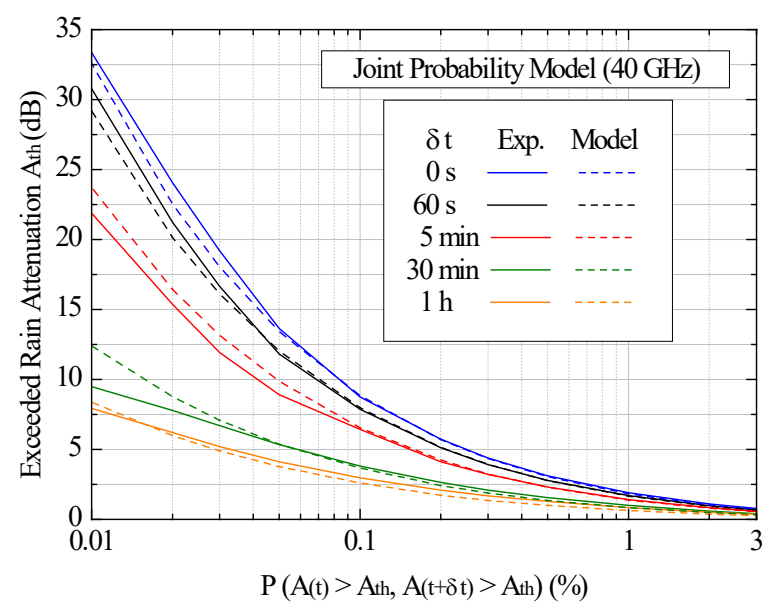

a)

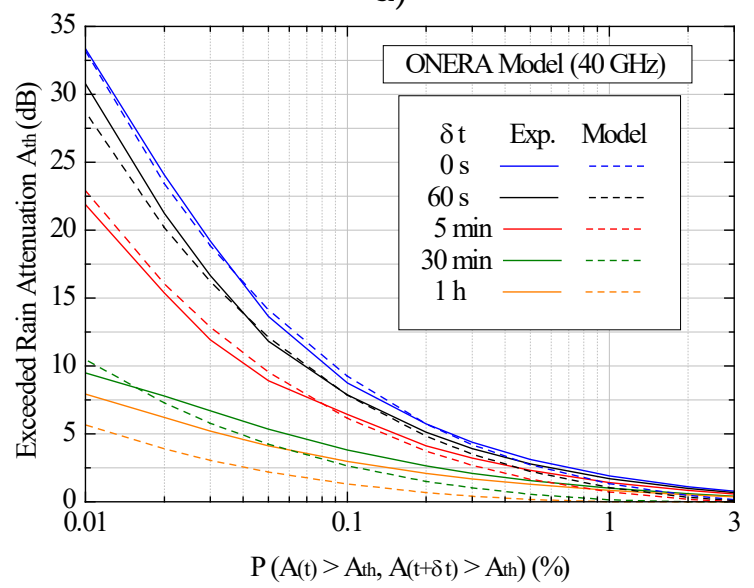

c)

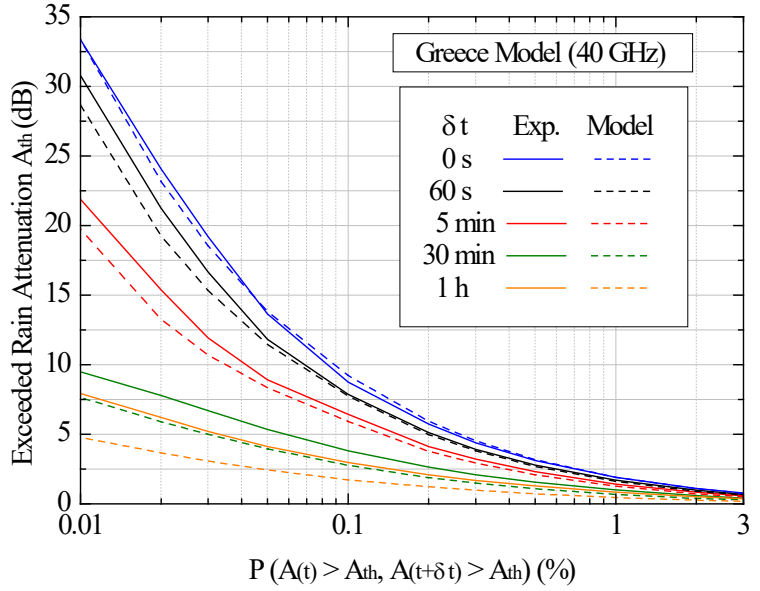

b)

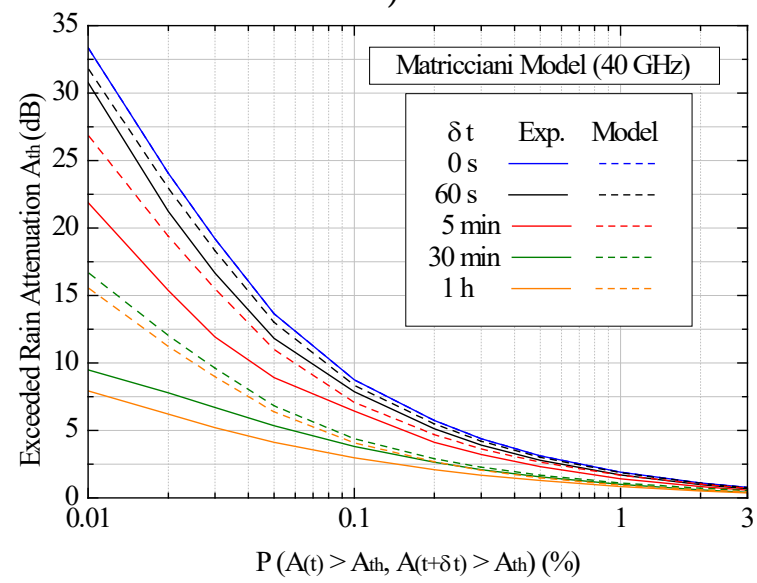

d)

Figure 5.25. TD models compared individually with the Q-band results for certain $\delta t$, a) Joint Probability, b) Greece, c) ONERA and d) Matricciani.

Figure 5.26 shows in a more compact manner the graphical comparison for some selected delay values. Model performances are usually worse for longer delays, with the Matricciani TD model (Matr. in Figure 5.26) being the best one for the longest selected delay (30 minutes).

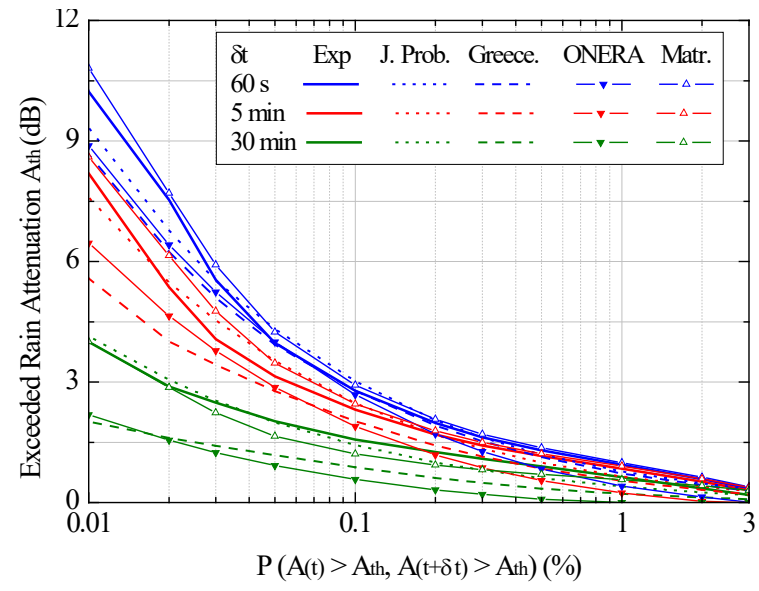

a)

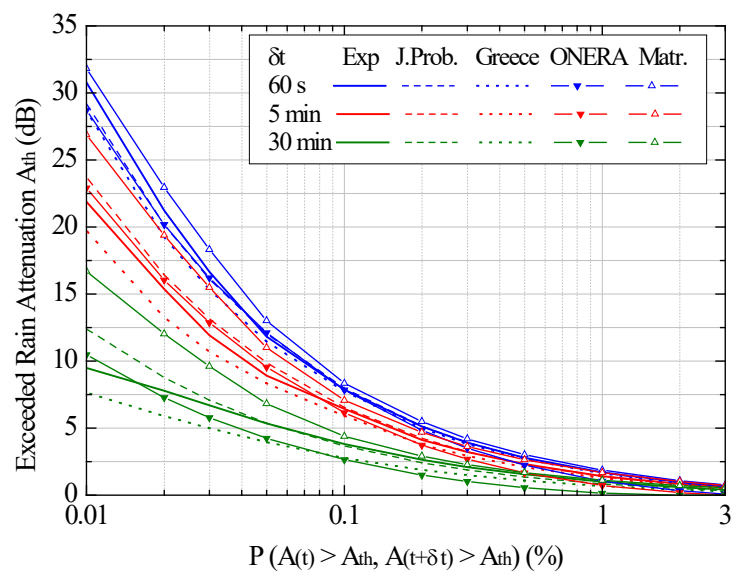

b)

Figure 5.26. TD models compared with the results, a) Ka-band and b) Q-band. 
Using the predictions of the TD models, an error analysis was carried out to assess their accuracy with the measurements. The test variable of (5.15) is used to this end, as was done in $[\mathbf{1 3 1}]$ (this is done since the ITU-R does not recommend any specific testing variable for time diversity):

$$
\varepsilon(\delta t, p)=100 \cdot \frac{G_{p r e d}(\delta t, p)-G_{\text {meas }}(\delta t, p)}{A_{\text {meas }}(p)}
$$

with $G_{\text {pred }}$ and $G_{\text {meas }}$ being the predicted and the measured time diversity gains, respectively, obtained by using their corresponding attenuation values in (2.49).

The RMSs of the test variables for the $0.01-3 \%$ interval are given in Table 5.15 for time delays of up to 1 hour. The Joint Probability model gives the best results (as can be expected from a fitting procedure). The Matricciani TD model behaves particularly well for both bands. The Greece and ONERA models, on the other hand, give better results for the Qband. For both experiments, all models show in general an increase in the errors when the time delay increases.

Table 5.15. RMS values (in \%) for TD models

\begin{tabular}{c|cccc|cccc}
\hline \hline & \multicolumn{4}{|c|}{ Ka-band } & \multicolumn{4}{c}{ Q-band } \\
\multicolumn{1}{c|}{} & $\begin{array}{c}\text { Joint } \\
\text { Prob. }\end{array}$ & Greece & ONERA & Matricciani & $\begin{array}{c}\text { Joint } \\
\text { Prob. }\end{array}$ & Greece & ONERA & Matricciani \\
\hline $10 \mathrm{~s}$ & 1.62 & 5.17 & 4.93 & 2.18 & 2.13 & 2.98 & 3.19 & 2.27 \\
$30 \mathrm{~s}$ & 2.18 & 7.91 & 7.02 & 4.58 & 2.50 & 4.11 & 3.67 & 5.17 \\
$60 \mathrm{~s}$ & 2.13 & 10.46 & 9.05 & 6.02 & 3.10 & 5.03 & 3.88 & 7.85 \\
$180 \mathrm{~s}$ & 4.20 & 15.34 & 12.82 & 8.98 & 4.73 & 6.76 & 4.56 & 12.50 \\
$300 \mathrm{~s}$ & 5.42 & 18.50 & 15.81 & 9.22 & 6.58 & 7.79 & 6.03 & 14.39 \\
$600 \mathrm{~s}$ & 7.41 & 22.42 & 20.90 & 9.36 & 8.14 & 9.58 & 8.99 & 15.02 \\
$1800 \mathrm{~s}$ & 11.43 & 28.55 & 30.14 & 12.90 & 5.80 & 13.91 & 15.25 & 12.48 \\
$3600 \mathrm{~s}$ & 13.98 & 31.22 & 33.59 & 14.77 & 5.27 & 16.84 & 19.38 & 14.10 \\
Ave. & 6.05 & 17.45 & 16.78 & 8.50 & 3.01 & 4.73 & 8.12 & 10.47 \\
RMS & \multicolumn{7}{|c|}{} \\
\hline \hline
\end{tabular}

\subsection{Orbital diversity results and modeling}

Since there is only one link per frequency and for an OD analysis two links at the same frequency are needed, a procedure is conducted in order to evaluate the OD in the Q-band with the available experiments.

At a first stage, the two frequency scaling methods of Recommendation ITU-R P. 618-13 [16] were applied to the experimental KA-SAT attenuation distribution to scale it to the Alphasat frequency. Here it is convenient to point out that, since both experiments share the same weather conditions and the analyzed time of 4-year concurrent period is relatively long, similar distributions are expected when both are referenced to the same frequency by scaling one of them. The remaining difference can be attributed to the different elevation angles, in which the KA-SAT-scaled distribution must go "below" the Alphasat distribution because KA-SAT has a higher elevation angle. However, when the frequency-scaled KA-SAT 
distributions resulting from the ITU-R methods are compared with the Alphasat distribution, this expectation is not fulfilled, i.e. in both cases the expected behavior is only followed for probabilities of occurrence of less than $0.05 \%$; for higher probabilities, the scaled distributions obtained by the two methods give a much higher attenuation than the Alphasat distribution.

To overcome this inconsistency, the following procedure was applied [226]. First, the KASAT distribution was scaled in elevation using a factor dependent on both elevation angles: $\sin \left(41.4^{\circ}\right) / \sin \left(34.5^{\circ}\right)$, in order to remove this effect from the elaboration of the frequencyscaling procedure (in Figure 5.27 the KASAT corrected curve shows the result of this step). Once both distributions are available for the same elevation angle, then the frequency-scaling fourth-degree polynomial that best approximates the Alphasat distribution from the KA-SAT one (in the range of probability of 0.01 to $3 \%$, up to $35 \mathrm{~dB}$ of attenuation and giving a RMS value of $4.55 \%$ of the testing variable for attenuation defined in [221]) is found. This empirical polynomial is shown in (5.16) and its result is displayed in Figure 5.27 with the Fitting ALP-KASAT curve (dotted line with green asterisks).

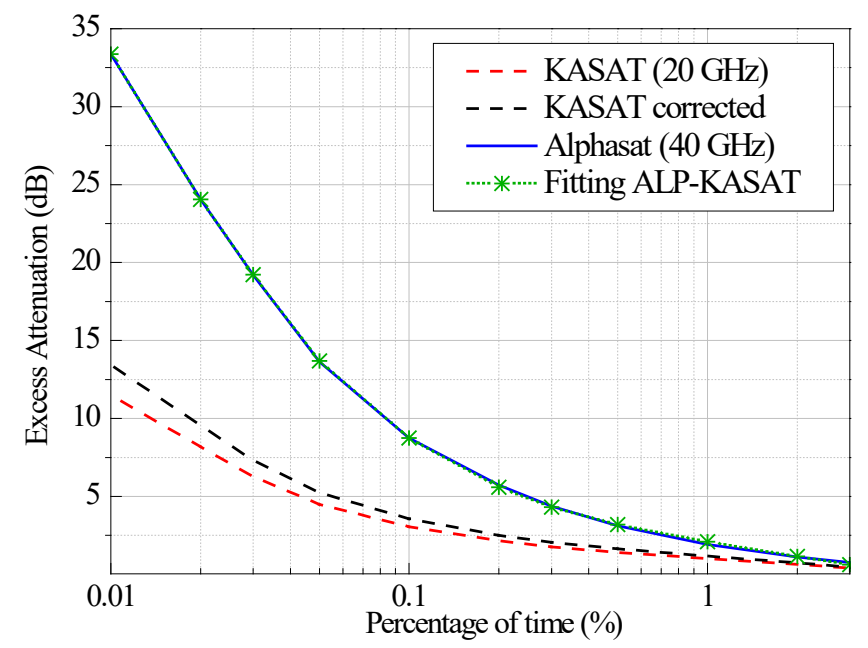

Figure 5.27. Curves for the frequency scaling procedure used in the OD analysis.

$$
A_{\text {sca }}=0.0023 \cdot A_{K a}^{4}-0.0619 \cdot A_{K a}^{3}+0.5087 \cdot A_{K a}^{2}+1.3445 \cdot A_{K a}-0.1033
$$

Later, the obtained polynomial was applied to the KA-SAT experimental attenuation series $A_{K a}$ obtaining $A_{s c a}$ : a KA-SAT time series scaled to $40 \mathrm{GHz}$. $A_{s c a}$ has the same elevation angle as $A_{K a}$. The frequency-scaled distribution $A_{s c a}$ can be seen in Figure 5.28 in solid red line as Kasat sca $(40 \mathrm{GHz})$. In the figure, there are also represented for comparison purpose the Alphasat (Alphasat $(40 \mathrm{GHz})$ ) and KA-SAT (KASAT (20 GHz)) excess attenuation distributions, the experimental joint probability of the simultaneous exceedance of the same attenuation level in the distributions of both links at the same Q-band frequency (Joint Distribution curve in green color), the OD model predictions of sub-section 2.6.2 (Matricciani OD Model and NTUA Model) and the statistical independency distribution of (5.14) (Stat. Indep.).

The OD gain is estimated using the Alphasat link as a reference and comparing its attenuation distribution to the joint probability of the simultaneous exceedance of the same attenuation levels in both links (Joint Distribution curve). From Figure 5.28, it can be seen 
that OD gain is not too high, as can be expected from the small azimuth separation between the links, but it can amount up to approximately $10 \mathrm{~dB}$ for the lowest probabilities considered. On the other hand, it is very small for probabilities of more than $1 \%$.

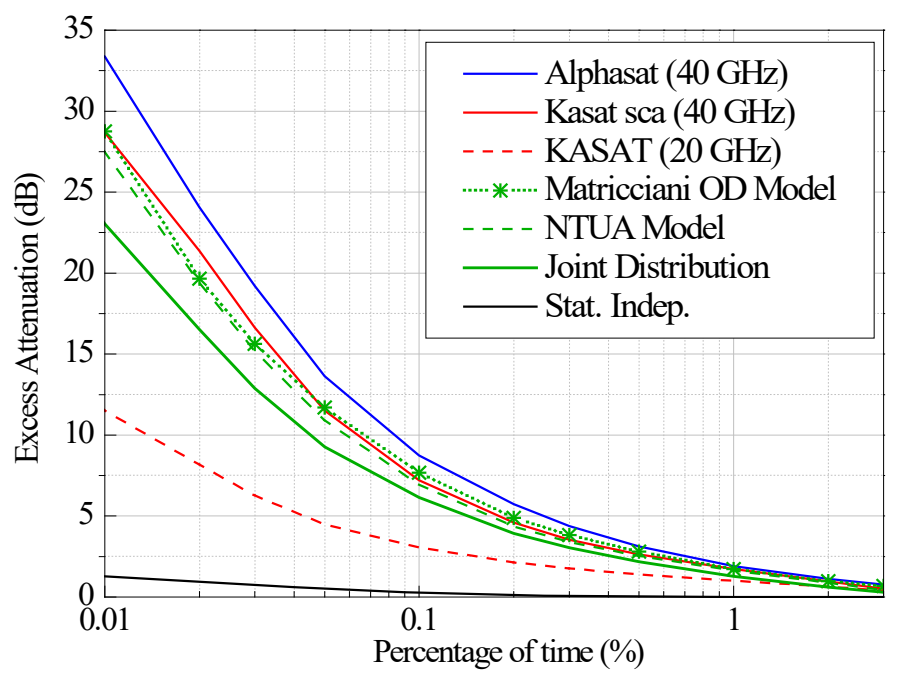

Figure 5.28. Comparison between the experimental distributions and OD models.

The RMS value for the Matricciani OD model is $21.80 \%$ and for the NTUA is $14.58 \%$, both for the range of 0.01 to $1 \%$ of the time. Models have a higher deviation from the joint probability distribution at percentages of time lower than $0.1 \%$, and both are more close to the KA-SAT scaled curve than to the joint one. It must be pointed out that both use parameters that are not available for Madrid, then the values given in the references were used. This may be one of the possible reasons of the major deviations.

Figure 5.29 presents a comparison between the obtained OD gain and TD gains for several time delays.

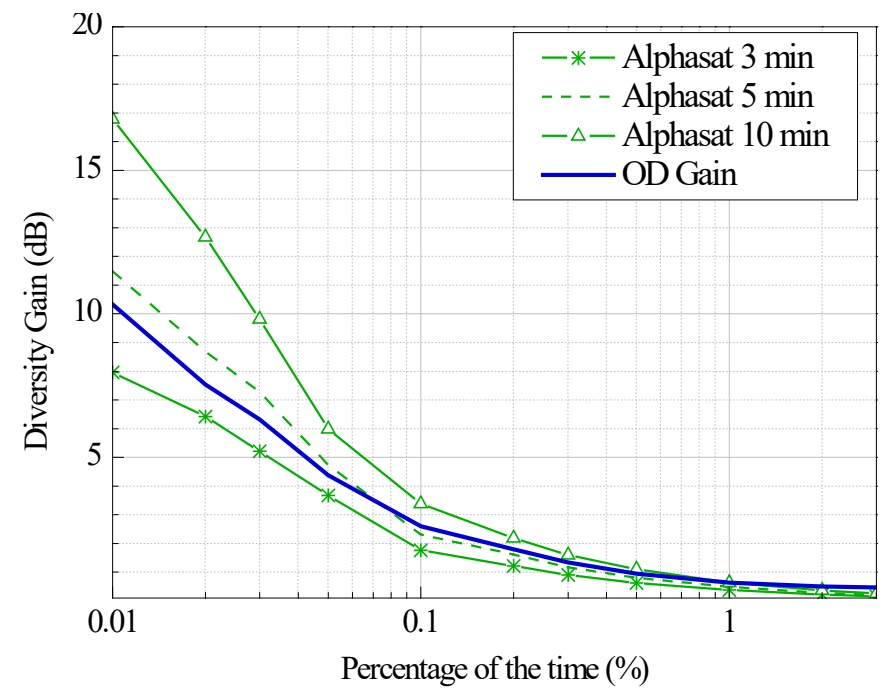

Figure 5.29. Comparison between OD gain and TD gains for $\delta t$ of 3,5 and 10 minutes.

It can be seen that the OD gain obtained with this $18.1^{\circ}$ scheme approximates the TD gain with a time delay between 3 and 5 minutes. In a previous work [226] the best similarity was obtained for a 3-minute delay. 


\subsection{Variability}

\subsubsection{First-order statistic variability}

The variability results on rainfall rate, Ka-band attenuation (both for the 4- and 5-year periods of measurements) and Q-band attenuation are shown in Figure 5.30, together with the ITU-R prediction [154] of (2.72) and a previous rainfall rate variability result for a 16year period presented in [228] (16 years of rain data) included as reference for comparison purposes. The probability $p$ is given in $\%$.

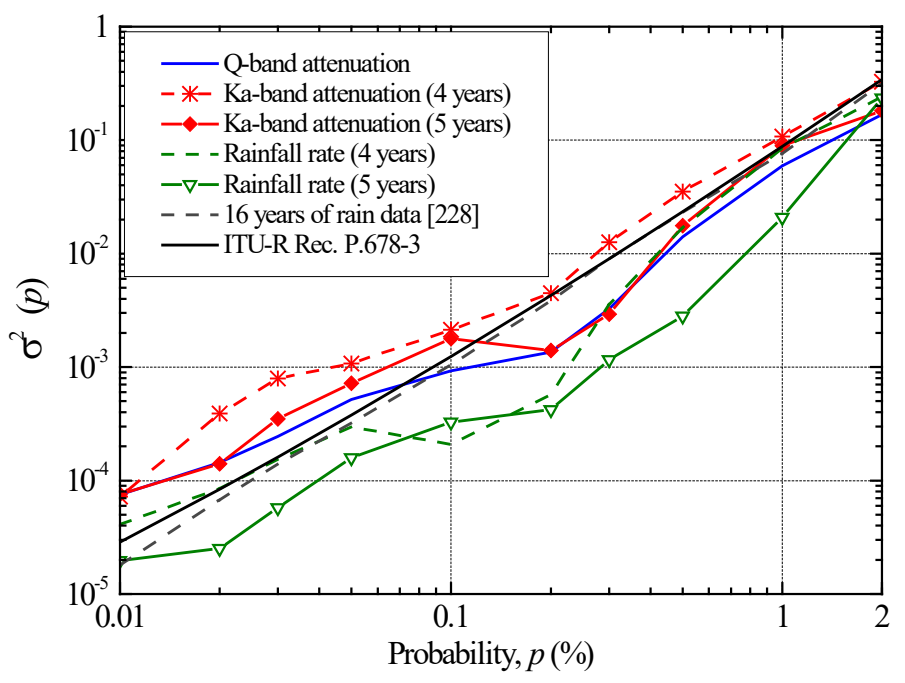

Figure 5.30. Variance $\sigma^{2}(p)$ for probabilities $p$ (in $\%$ ).

The relative differences between the model prediction and the experimental results have been calculated by using:

$$
\varepsilon_{\sigma}(p)=\frac{\sigma_{p}(p)-\sigma_{m}(p)}{\sigma_{m}(p)}
$$

$\sigma_{p}(p)$ and $\sigma_{m}(p)$ being the predicted and the measured variance (either for rainfall rate or attenuation) for probabilitiy $p$, respectively. The lowest RMS value of these differences is obtained for the 16-year curve (0.23) as can be expected from such a long period, followed by the Ka-band (0.49) and Q-band (1.02) attenuations for the 4-year period, the Ka-band attenuation for the 5-year period (1.03), the rainfall rate for the 4-year concurrent period (2.63) and the highest difference was obtained for the rainfall rate for the 5-year period (4.61).

Having $\sigma^{2}(p)$, the ratio of the standard deviation with respect to the mean probability $\sigma(p) / p$, can be derived. This ratio is depicted in Figure 5.31. It provides information about how much the probability of exceedance for a given threshold (either for rainfall rate or attenuation) deviates from the mean value in relative terms. It is seen that the model predicts a decrease of variability in relative terms with the increase of the probability; this is roughly seen for all the experimental curves. The 16-year is the closest curve (and also the smoothest) to the model predictions, which seems to represent well the long-term variability of the distributions, at least for rainfall rate. For the analyzed periods, the differences 
between model and results can be classified as moderate (predictions and results are in the same order of magnitude).

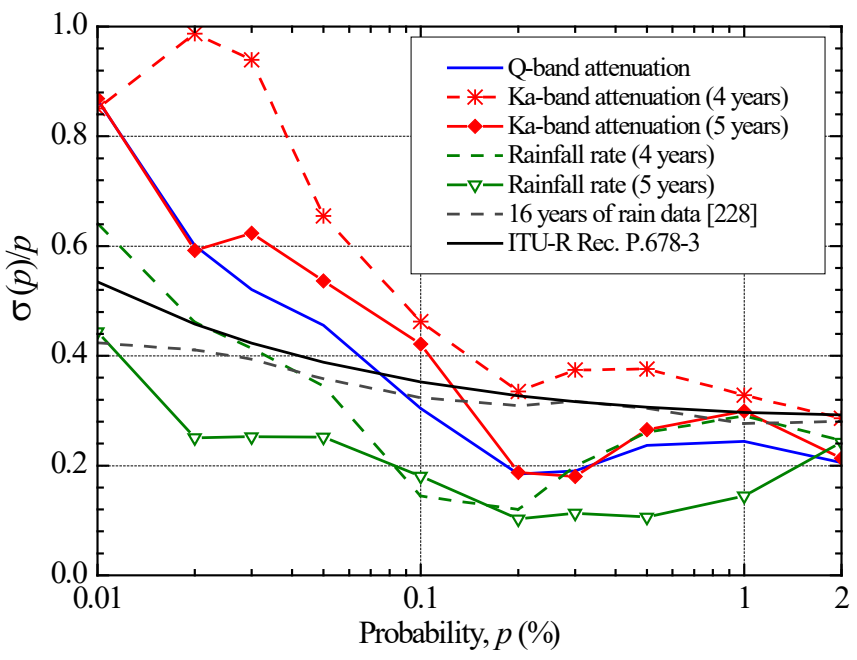

Figure 5.31. Variability of the statistics depending of probability $p(\%)$.

\subsubsection{Second-order statistic variability}

\section{Fade duration variability}

The fade and inter-fade duration variability are evaluated by using (2.81) with the respective (2.82) for each statistic for the 4-year concurrent period.

From Figure 5.32 a first idea of the variability of the number of fade events among the different years can be obtained. In both cases the number of events for each attenuation threshold is quite similar for the different years. Nevertheless, higher variations are observed for higher thresholds, especially for the Ka-band case.

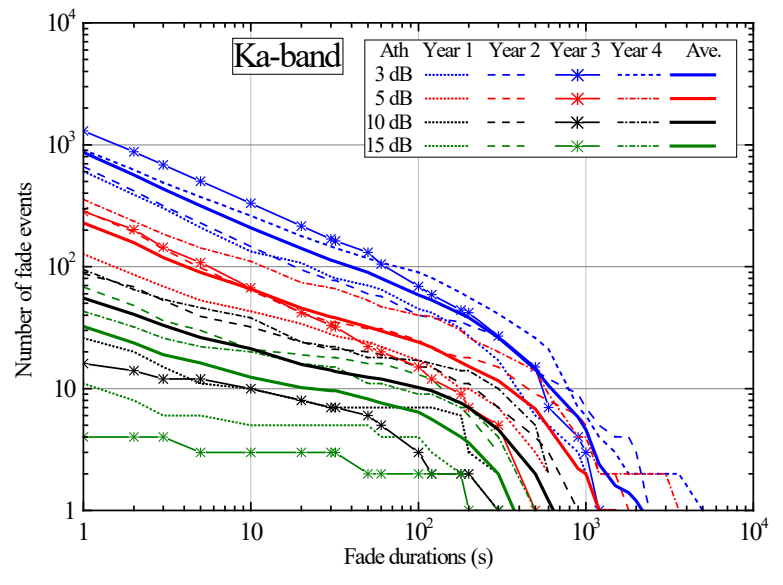

a)

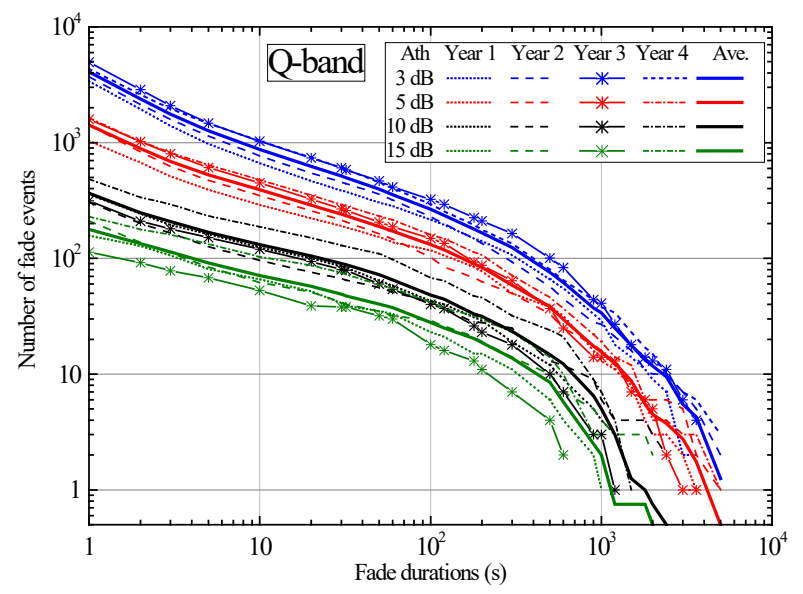

b)

Figure 5.32. Number of FD events for the individual years for a) Ka- and b) Q-bands.

For the fade duration statistics (i.e. fade duration number $N(D)$, total fading time $T(D)$, probability of occurrence $P(D)$ and total fraction of fade time $F(D)$ ), variability results are given in Figure 5.33 using now the parameter defined by (2.81). 

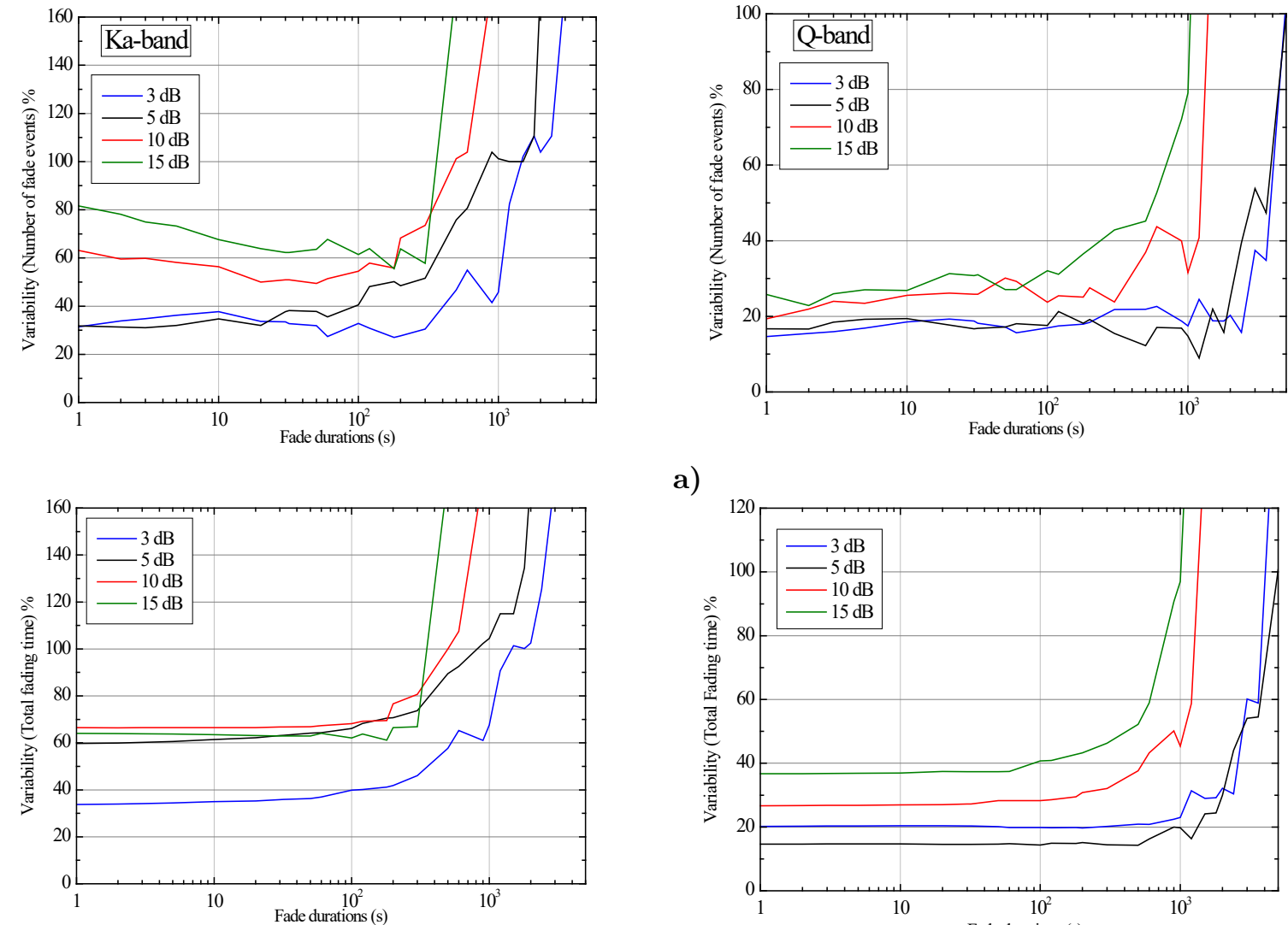

a)

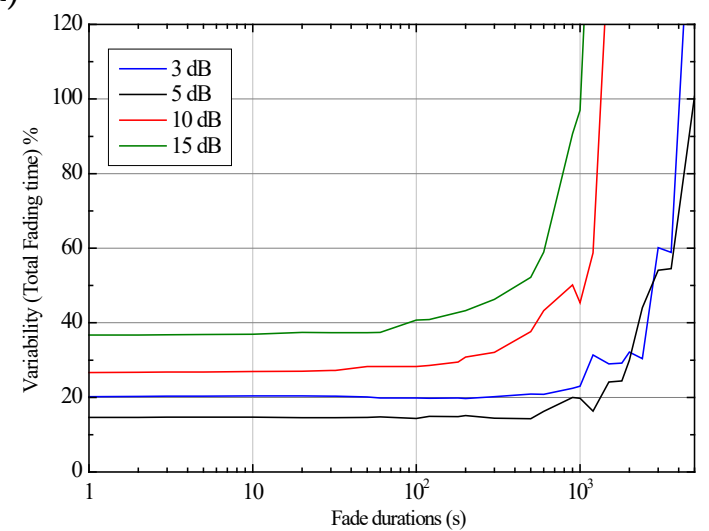

b)
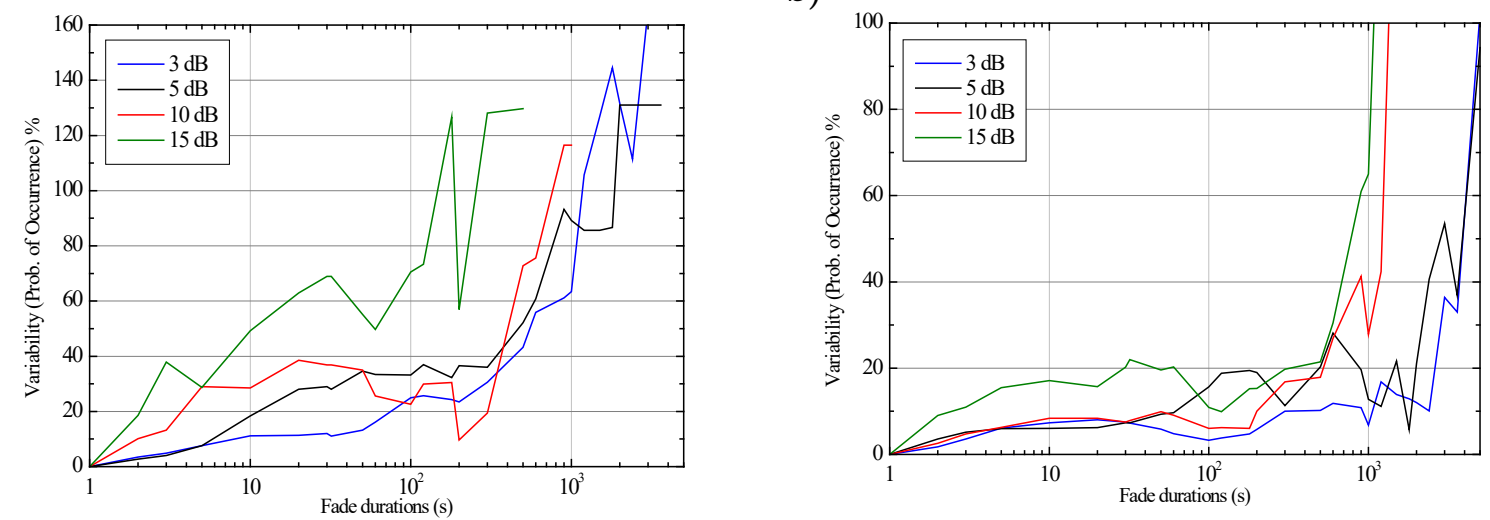

c)
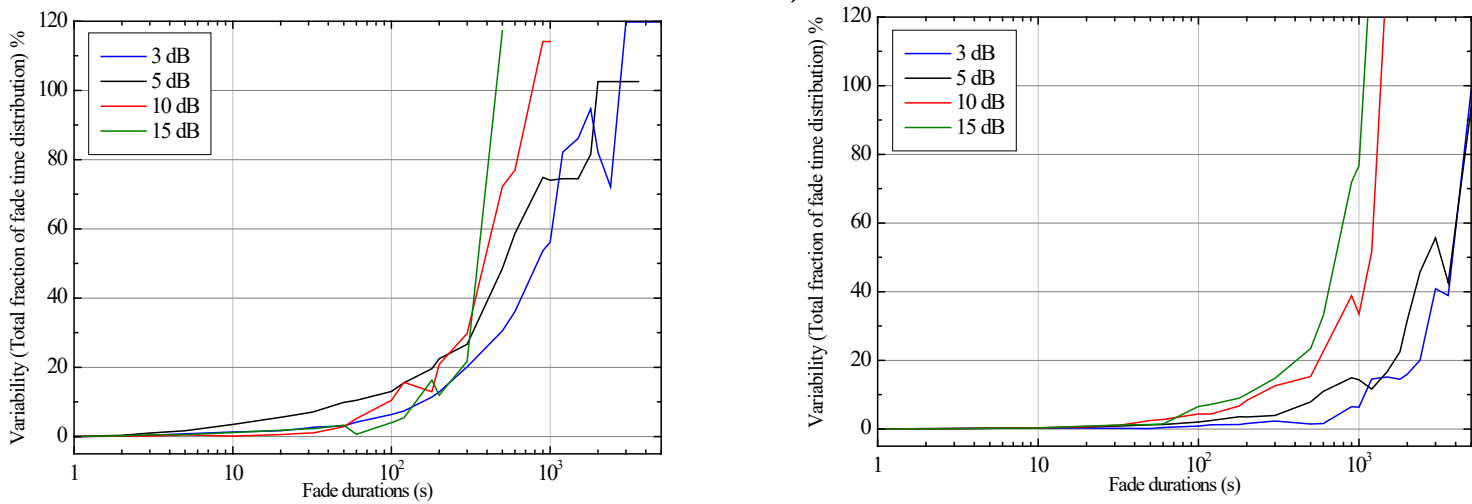

d)

Figure 5.33. Ka- (in the left-hand) and Q-band (in the right-hand) FD variability for a) number of events, b) total fading time, c) probability of occurrence and d) total fraction of fade time. 
A quite stable behavior can be seen up to $300 \mathrm{~s}$ for the Ka-band and up to $400 \mathrm{~s}$ for the Q-band. For values higher than these, the behavior of all curves is more erratic, because the number of events exceeding those long durations becomes very small, especially for the higher attenuation thresholds.

The results confirm that the variability is higher for the Ka-band case. This is related to the fact that the probability (or time) of exceeding a given attenuation threshold is lower at lower frequencies. On the other hand, if a comparison between the $15 \mathrm{~dB}$ curve of Q-band and the $5 \mathrm{~dB}$ curve of Ka-band is carried out, a similar trend is obtained (at least up to 1000 s since for longer durations the number of events is too small). The same can be said about the comparison of the curves for $10 \mathrm{~dB}$ in the Q-band and $3 \mathrm{~dB}$ in the Ka-band. This can be attributed to the similar probabilities of exceeding these pairs of thresholds in the two bands (i.e. an outage of a lower attenuation threshold, 3 or $5 \mathrm{~dB}$, in the Ka-band shares a similar probability of occurrence with an outage of a higher attenuation threshold, 10 or $15 \mathrm{~dB}$ in the Q-band).

\section{Inter-fade duration variability}

In an analogous manner, the variation of the number of IFD events is shown in Figure 5.34. Again, the larger variations are seen for the Ka-band results at $15 \mathrm{~dB}$ attenuation threshold.

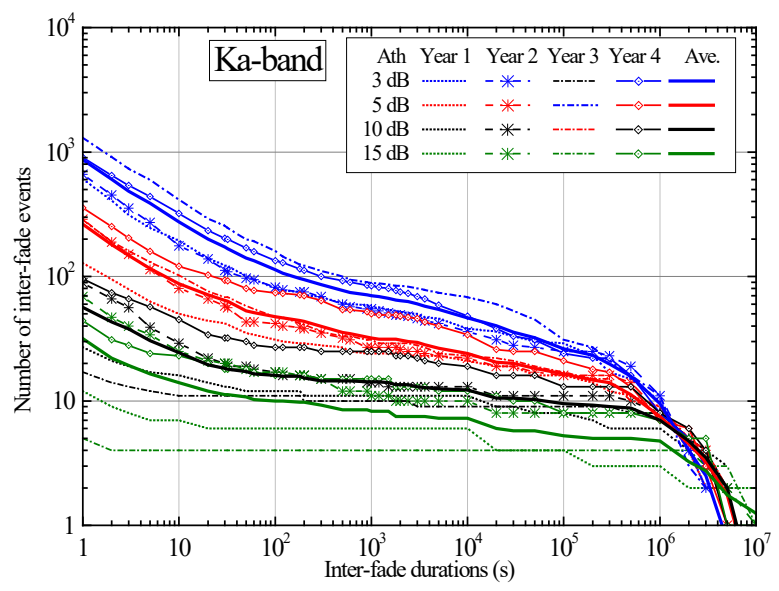

a)

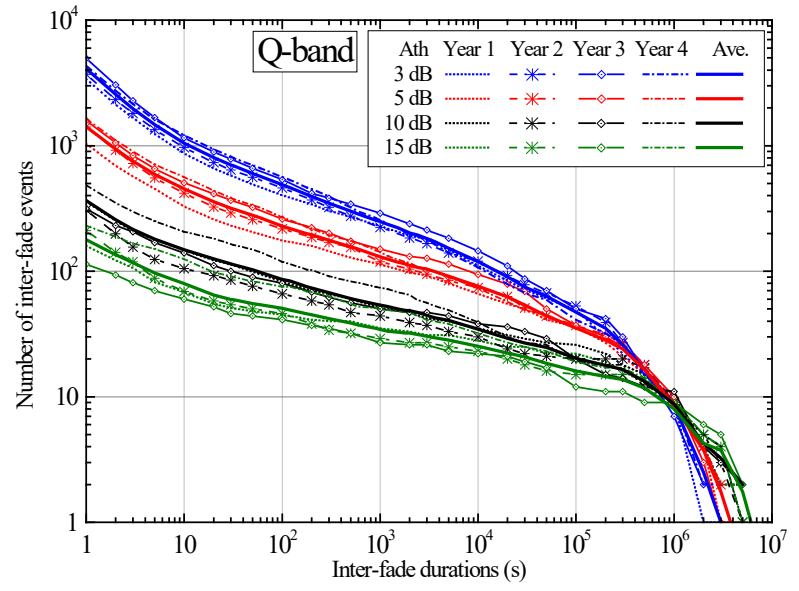

b)

Figure 5.34. Number of FD events for the individual years for a) Ka- and b) Q-bands.

The variability of the number of inter-fade events, the total inter-fading time, the probability of occurrence and the total fraction of inter-fade time are represented in Figure 5.35 for the 4-year concurrent period of the two experiments. 

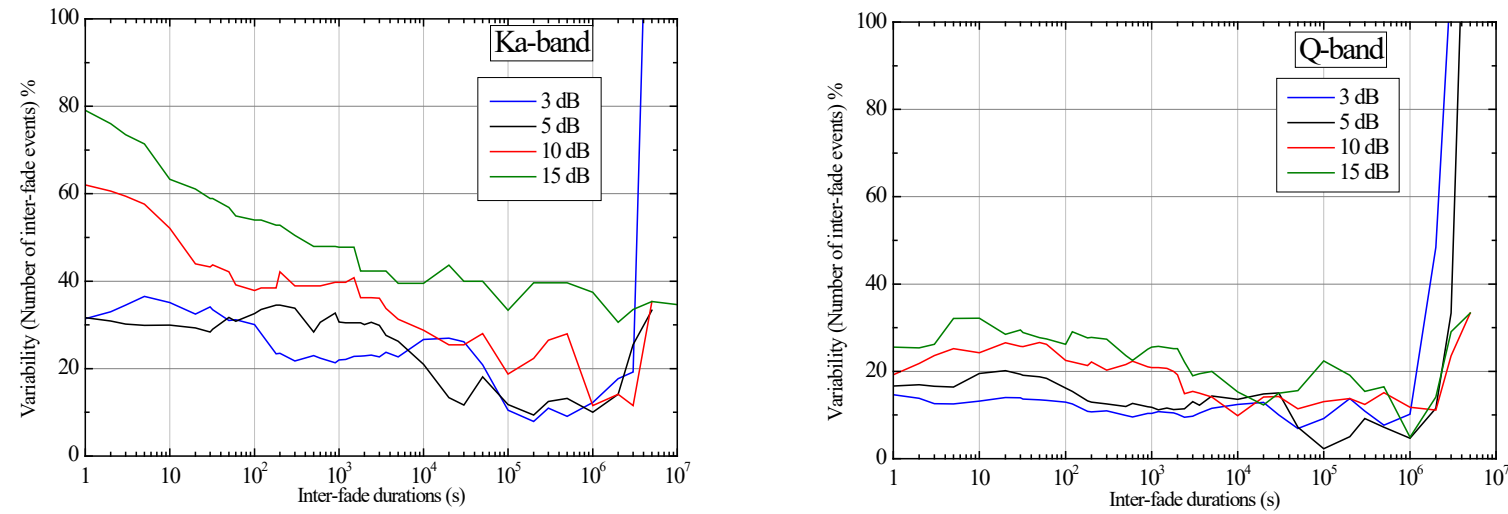

a)
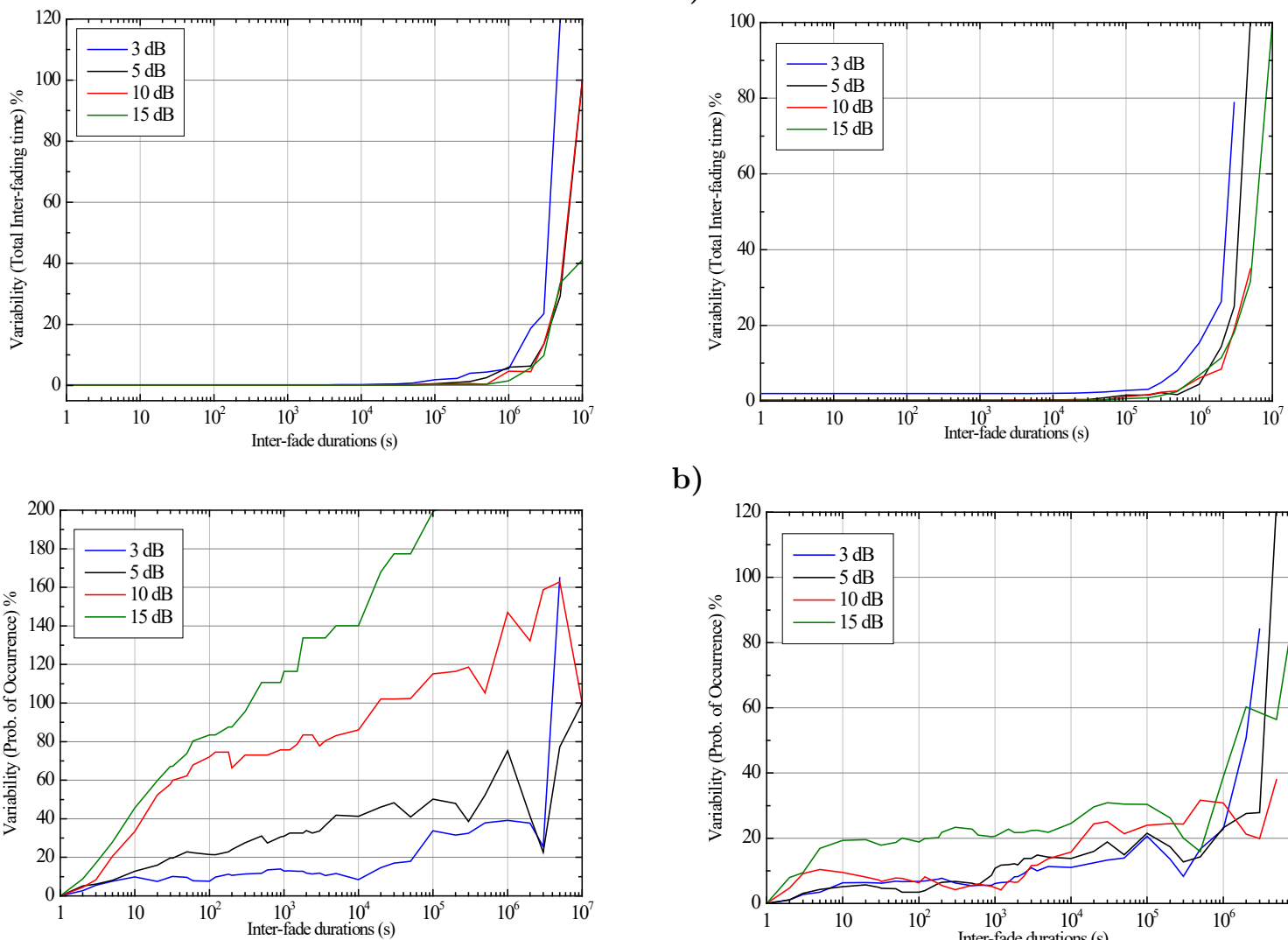

b)

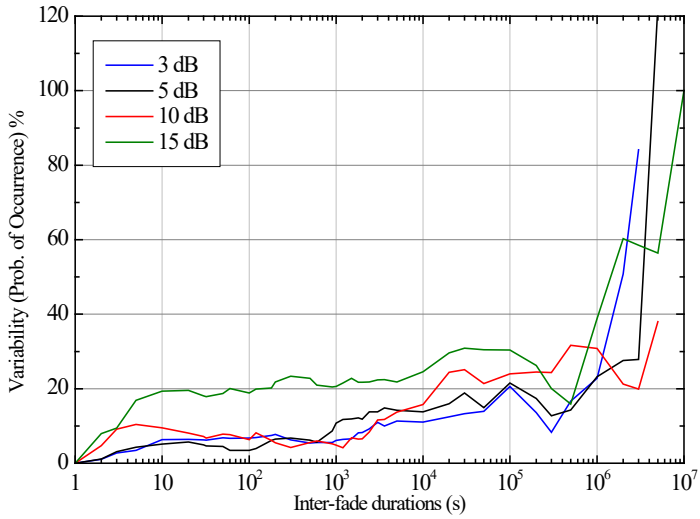

c)
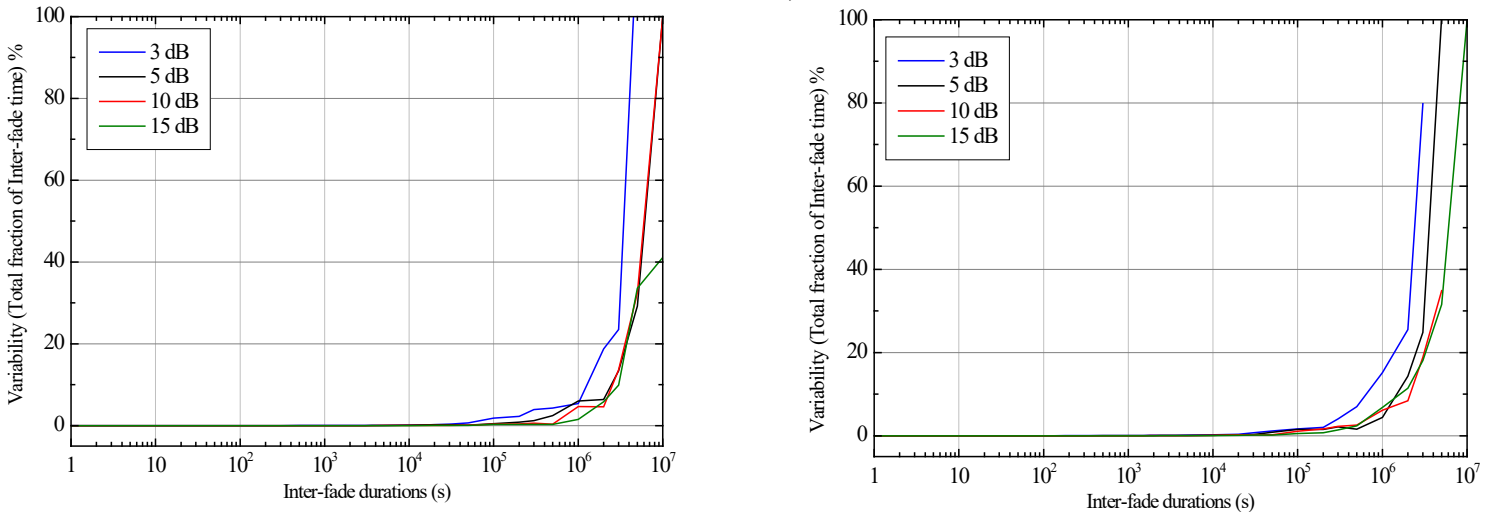

d)

Figure 5.35. Ka- (in the left-hand) and Q-band (in the right-hand) IFD variability for (a) inter-fade duration number, b) total inter-fading time, c) probability of occurrence and d) total fraction of inter-fade time. 
The same trend holds both for FD and IFD: overall, the highest variability is obtained for the Ka-band case, and for 10 and $15 \mathrm{~dB}$ thresholds. For each band, the variability for 3 and $5 \mathrm{~dB}$ is quite similar, having a more unstable behavior in the Ka-band. In the case of IFD, for durations longer than around $10^{6} \mathrm{~s}$ the statistical stability is worse due to the small number of events.

\subsection{Conclusions}

Regarding the obtained satellite propagation results, it is worth mentioning that all the presented analyses were possible due to the very high experimental availability, either for KA-SAT or Alphasat, in both cases higher than $97.6 \%$ of the time periods.

\section{Rainfall rate}

Rainfall rate modeling is one of the most important issues in satellite propagation. Although this is not the main goal of this work, it is clear that rainfall rate distributions are a necessary first-order statistic affecting almost all the subsequent results.

The three analyzed models give very good approximations for the two derived rainfall rate distributions (for a four and a five year period, corresponding to the measurement periods of both experiments). It can be said that for Madrid, MORSE and the recently approved ITU-R model improve the prediction accuracy for the low probabilities (from 0.001 to $0.005 \%$ ) related to the occurrence of convective rain events. The higher deviations are in the medium probabilities (from approximately 0.005 to $0.03 \%$ ), for which the three models overestimate the experimental distributions. Also, a parameter to have into account is the percentage probability of rain in an average year $\left(P_{0}\right)$, which is overestimated in all cases, with the Rec. ITU-R P.837-6 model giving the best approximation in general.

\section{Attenuation}

Regarding attenuation results, these are also relevant first-order statistics. The excess attenuation time series are the foundation of all the later analyses. However, the focus of this research is not on this kind of statistic due to the higher amount of researches on this topic. Nevertheless, it can be concluded that the analyzed periods have a high month-to-month and year-to-year variation regarding excess attenuation for both frequencies, the higher variability is seen for probabilities of less than $0.05 \%$. As can be expected, higher attenuation values are obtained for the highest frequency.

Total attenuation results were gathered only for the Q-band (and presented in Annex 3) and are not so different from excess attenuation results, the difference is basically the gas attenuation, which is derived from GNSS data (other minor differences arise from the lack of GNSS data and the subsequent elimination of those periods in the analysis). Although this method only provides an approximation to gas attenuation, it is considered appropriate since it has been used for validating radiometer measurements with very good performance.

\section{Fade duration probability of occurrence}

On the other hand, since fade dynamics have been less addressed in the open literature, they have been studied more in depth in this work. Applying the ITU-R and CRC models for 
the FD probability of occurrence, it can be said that the ITU-R model gives the best overall approximations. The CRC predictions for the experimental conditions are rather poor in the Q-band and at higher attenuation thresholds in the Ka-band, giving the best approximations at lower thresholds ( 3 and $5 \mathrm{~dB}$ ) in the Ka-band. It seems that neither the used Pwr+Log combination of the ITU-R model nor the Log+Log combination of the CRC model describe the results accurately. Maybe their coefficients are not optimized for the Madrid case.

In order to analyze means for model improvement, a fitting effort has been carried out with four combinations of distributions ( $\log +\log$, Pwr $+\log , \operatorname{Exp}+\log$, Exp+Exp+Exp), obtaining the best results for the two log-normal distribution (Log+Log); a model improvement could be possible if some work oriented to derive better coefficients is done - a task that may need a very large amount of data for several regions and the analysis of correlations with different climatological parameters. From the fitting results, it can be concluded also that the ITU-R P. 1623-1 model (based on a Pwr+Log combination), CRC model (based on a Log+Log combination) and Bråten model (based on a three exponential combination) do not have their coefficients optimized for the conditions in Madrid, having very high deviations from the presented results.

\section{Fraction of fading time}

Regarding the fraction of fading time, the ITU-R model gives better predictions for the Ka-band than for the Q-band. In both cases there is a point in which the distribution behavior changes and this is not followed by the ITU-R model: the fraction of fading time decreases with the increase in the threshold, but this trend does not hold experimentally for durations shorter than approximately $300 \mathrm{~s}$ for Ka-band (similar results have been obtained in other Ka-band measurements) and approximately $150 \mathrm{~s}$ for Q-band.

The presented procedure (UPM procedure) gives very good results compared with those obtained from the total fraction of fading time fitting. It estimates the total fraction of fading time distributions using the coefficients obtained by the $\log +\log$ probability of occurrence fitting. The procedure relies on the physical basis that the total fading time depends on the distribution of the number of fade events. A drawback is that the accuracy decreases when the attenuation threshold increases.

\section{Inter-fade duration probability of occurrence}

In the case of IFD, the carried out modeling effort confirms several ideas that have been outlined by the author in previously published papers related with this research: the ONERA fitting procedure with a two log-normal function combination can estimate well the IFD probability of occurrence and inter-fading time if some modifications are made (i.e. using a value in the range of 900 to $1500 \mathrm{~s}$ as a limit between short and long IFD and setting up an upper limit of $10^{6} \mathrm{~s}$ to the IFD distributions). The limit between short and long IFD encompasses the value conventionally used to limit a rain event (approximately $20 \mathrm{~min}$ ), then, the first distribution describes the short IFD produced by scintillation and intra-event rain fluctuations and the second distribution fits the longer IFD associated to inter-event intervals. Also, it must be noticed that a change in the trend of the number of IFD events occurs around $10^{6} \mathrm{~s}$ (approximately 11 days) either for Ka- or Q-band results. 
If IFD longer than $10^{6} \mathrm{~s}$ must be described, then it seems necessary the use of a third lognormal distribution, as the USA model does (although, the predictions of this model do not work well compared with the collected data). However, durations longer than $10^{6} \mathrm{~s}$ may have a low practical importance and better accuracy achieved in this range of IFD may not compensate the increase in the model complexity.

\section{Inter-fading time}

The more adequate combination for modeling the inter-fading time distribution was a two log-normal function combination using the ONERA-modified fitting procedure. Again, longer durations are the more difficult to model, but still, very good approximations are obtained up to $10^{6} \mathrm{~s}$. These distributions, on the other hand, do not have a point in which they change their behavior: the fraction of time is always higher for the higher attenuation and the distributions decrease in a smoothest way. This fact may be taken as an advantage for future model efforts.

\section{Fade slope}

The ITU-R fade slope model gives good predictions at both frequency bands. This model demonstrates again a very good performance. Nevertheless, performance decreases for longer fade slope intervals and attenuation thresholds, although lower RMS values are obtained for lower time intervals (2 and $10 \mathrm{~s})$ with the highest attenuation $(15 \mathrm{~dB})$.

\section{Time diversity}

The application of this diversity technique with the experimental data demonstrates that the diversity gain for moderate time delays (up to $10 \mathrm{~min}$ ) is not negligible. Also, a higher gain can be obtained at the higher frequency; this may be conditioned to the rain event durations and the strong attenuations that they produce at such frequency. Waiting some minutes to re-send the information may serve to avoid a very deep fade event (more prone to occur in the Q-band than in the Ka-band).

Four TD models have been analyzed, all of them providing good approximations of the data, although they have not been tested globally. The Joint probability model was applied more as a fitting procedure than as a prediction model, since its coefficients, not available for Madrid, were derived from the measurements. The Matricciani TD model has a very good behavior for both bands. Altogether, the predictions are better for the Q-band than for the Ka-band.

\section{Orbital diversity}

The OD analysis in the Q-band was possible due to the development and application of a procedure that overcomes the difference in elevation angle and frequencies between experiments. The ITU-R frequency scaling methods were not used in this OD study due to their inconsistency. The two tested OD models give moderate approximation, maybe conditioned to the small aperture angle (of around $18^{\circ}$ ) and that they have been developed for lower frequencies (up to the Ka-band). Also, the OD gain is very similar to the possible TD gains obtained with a delay between 3 and 5 minutes. Then, OD and TD can be used in a complementary manner, depending on the delay that can be tolerated by the application. 


\section{Variability}

Year-to-year variability is seen along all results. The rainfall rate and attenuation variability were compared with the ITU-R model (using the $\sigma^{2}$ parameter) and a previous result for a 16-year period of rainfall rate measurements. It is interesting to notice that the rainfall rate statistic for the 5-year period has the highest variability. Overall, the analyzed statistics have variability in the same order of magnitude as the ITU-R model, and follow in absolute terms the model decreasing behavior with the increase of the probability.

Second order statistics variability has been assessed with a different method. Altogether, yearly variability increases for higher thresholds, since events producing the higher attenuation are scarce and then the individual events have more weight than events of lower attenuations (which are averaged out in a larger number). Also, FD and IFD variability have a similar order of magnitude, with the normalized distributions (probability of occurrence and fraction of fading/inter-fading time) having less variability than the absolute distributions (number of fade/inter-fade events and total time). 


\section{Millimeter-wave Indoor channel results}

\section{Introduction}

The results on millimeter-wave channel propagation in an indoor scenario are given in this chapter. For assessing the path loss behavior, measurements have been taken in the 26and 39-GHz frequency bands in LOS and NLOS conditions, with two antenna configurations: the horn-horn, using a directional horn antenna in both link ends, and the horn-omni, which uses a directional horn antenna at the transmitter and an omnidirectional one at the receiver side. Hereinafter the two configurations are named as Horn and Omni respectively. From the path loss results, CI, FI and ABG (only for a single frequency) fittings have been applied, and the obtained coefficients are compared with those of the propagation models presented in Chapter 3. Multi-frequency fittings have not been used, since two appears to be a very low number of frequencies for obtaining frequency-dependent coefficients that must hold for a wide range.

Furthermore, $2 \times 2$ MIMO measurements at $39 \mathrm{GHz}$ are also presented. In this case, four omnidirectional antennas and a mechanical switching device are used. The measurements have been classified in LOS and NLOS and their statistical behavior is assessed by means of the condition number of the derived $\mathbf{H}$ matrices. 


\subsection{Path loss results and modeling}

\subsubsection{LOS results}

The measurements were taken in one of the hallways of the fourth floor of the building $\mathrm{C}$ of the ETSI Telecomunicación of the UPM, where the Radiocommunication laboratory of the GTIC Research Group is located. The floor plan is seen in Figure 6.1a. The corridor crosssection is $2.41 \mathrm{~m}$ wide by $2.89 \mathrm{~m}$ high, with wooden doors, walls of lightweight concrete and a false ceiling of gypsum along it (Corridor $A$ in Figure 6.1a is used for LOS measurements whereas Corridor $B$ is used together with the $A$ for NLOS measurements, both share the same structure characteristics). There are also emergency doors to the stairs which remain open all the time. Also, several small metallic trash bins, fire extinguishers, wooden shelves with crystals and a metallic water dispenser are placed along the corridor, as can be seen in Figure 6.1b.

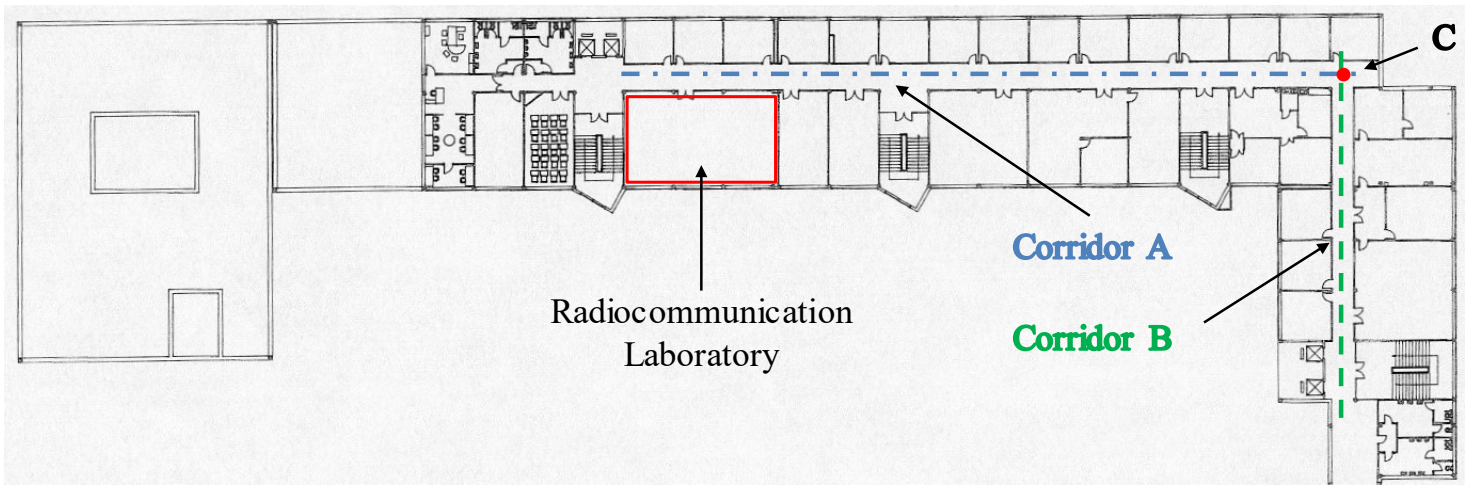

a)

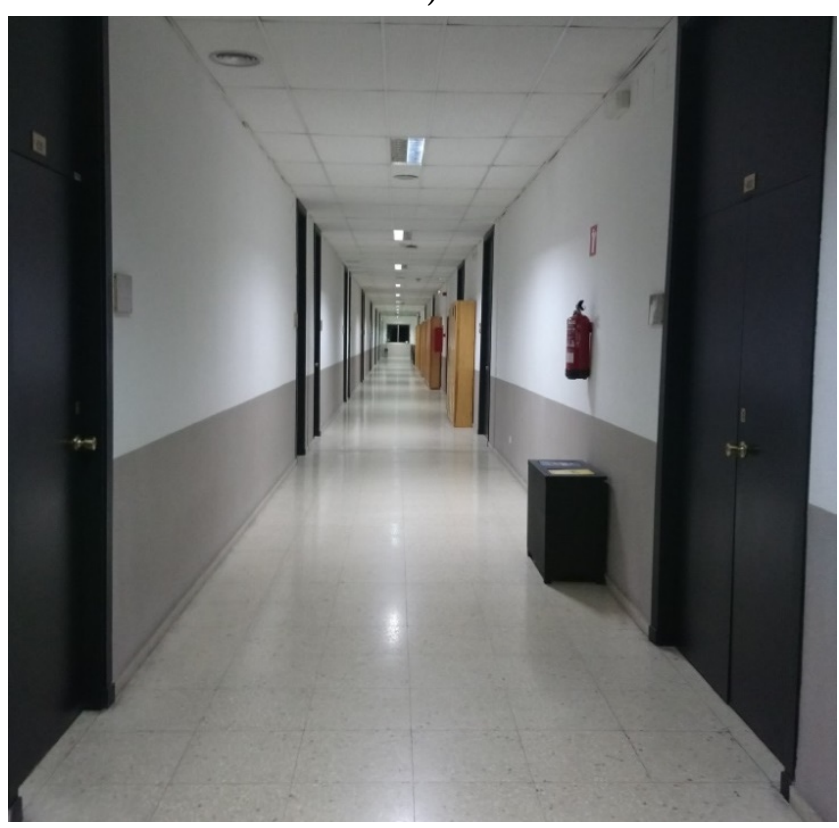

b)

Figure 6.1. Measurement scenario, a) floor plan and b) the Corridor where the LOS measurements were taken (Corridor A). 
The measured PL values in LOS condition for both configurations (Omni and Horn) are given in Figures 6.2 and 6.3 for 26 and $39 \mathrm{GHz}$ respectively. The black dashed line corresponds to the free space path loss (FSPL) at each frequency. The number of measurements (Meas.) at $26 \mathrm{GHz}$ is 283 , spanning from 3 to $50 \mathrm{~m}$, and 187 for $39 \mathrm{GHz}$, ranging from 3 to $60 \mathrm{~m}$. The minimum distance $\left(d_{\min }=3 \mathrm{~m}\right)$ was selected to warranty the far field conditions: it satisfies the $d_{\text {min }} \geq 2 D^{2} / \lambda$ and $d_{\text {min }} \gg \lambda$ expressions, with $D$ as the largest antenna dimension and $\lambda$ as the wavelength.

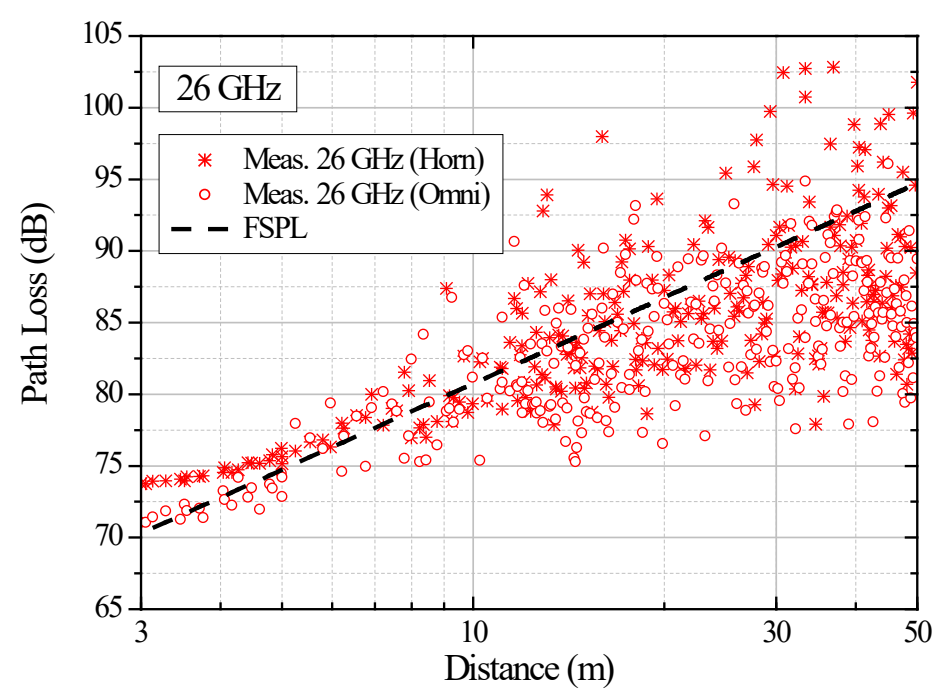

Figure 6.2. LOS results at $26 \mathrm{GHz}$.

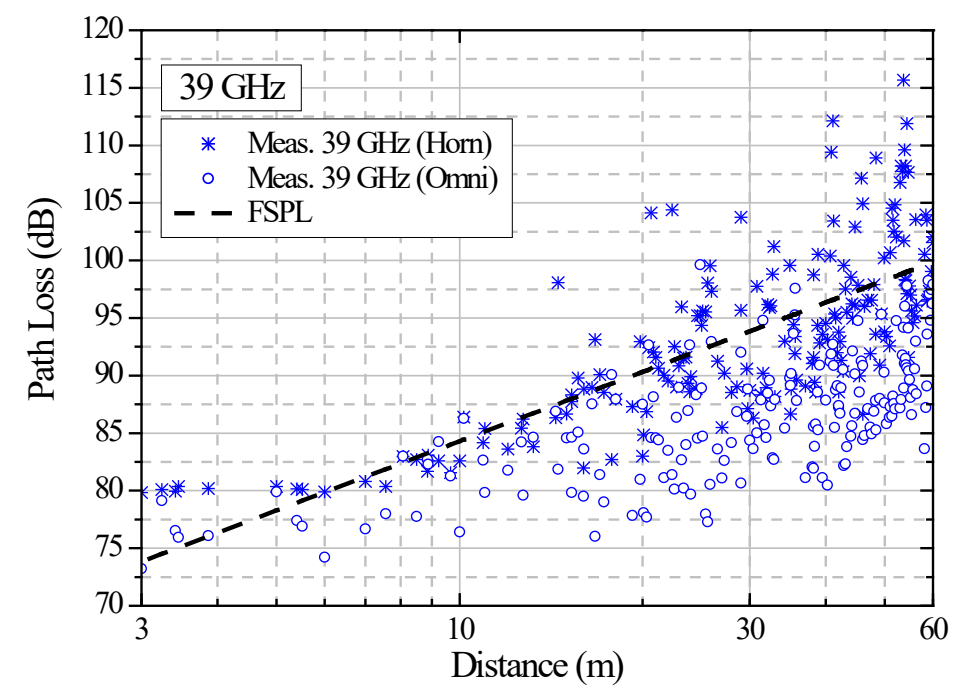

Figure 6.3. LOS results at $39 \mathrm{GHz}$.

It is seen that the Horn results are more tightly distributed around the FSPL line in both cases. In general, the Omni measurements (marked with circles) have a lower path loss compared with the Horn (asterisks), since they have a wider radiation pattern for collecting the received power from the different paths. As expected, the higher path loss corresponds to the higher frequency of $39 \mathrm{GHz}$. From the Friis's formula, under free-space conditions the path loss is $3.6 \mathrm{~dB}$ higher in $39 \mathrm{GHz}$ than in $26 \mathrm{GHz}$. Note that the plots represent the path loss once the difference in antenna gains has been removed. 


\subsubsection{LOS modeling}

\section{LOS path loss modeling}

The CI and FI and ABG (for a single frequency) fittings were applied to the LOS results, obtaining their respective coefficients, which are shown in Tables 6.1-6.2. The choice of these instead of more elaborated fittings such as the dual-slope versions is due to the fact that none of the different available path loss models use a dual slope for modeling indoor LOS conditions. For the CI and FI cases, the coefficients are also compared in Table 6.1 with those provided by some of the models given in section 3.3. Moreover, the anchor distance for the CI fitting has been optimized, being possible a comparison with the values obtained for the 1-m anchor (the default value).

Table 6.1. Comparison of coefficients between CI and FI fittings and LOS models

\begin{tabular}{|c|c|c|c|c|c|c|c|c|}
\hline \multirow[t]{2}{*}{ Experiment } & \multirow[b]{2}{*}{ Antenna } & \multirow[b]{2}{*}{ CI Anchor } & \multirow[b]{2}{*}{$F S P L(\mathrm{~dB})$} & \multicolumn{2}{|r|}{ CI } & \multicolumn{3}{|c|}{ FI } \\
\hline & & & & $n$ & $\sigma_{S F}(\mathrm{~dB})$ & $\alpha$ & $\beta$ & $\sigma_{S F}(\mathrm{~dB})$ \\
\hline \multirow{4}{*}{$26 \mathrm{GHz}$} & \multirow{2}{*}{ Horn } & $d_{0}=1 \mathrm{~m}$ & \multirow{4}{*}{60.74} & 1.86 & 4.62 & 67.17 & 1.39 & 4.38 \\
\hline & & $d_{o p t}=0.35 \mathrm{~m}$ & & 1.39 & 4.38 & & & \\
\hline & \multirow{2}{*}{ Omni } & $d_{0}=1 \mathrm{~m}$ & & 1.70 & 4.09 & 67.67 & 1.19 & 3.77 \\
\hline & & $d_{o p t}=0.26 \mathrm{~m}$ & & 1.19 & 3.77 & & & \\
\hline \multirow{4}{*}{$39 \mathrm{GHz}$} & \multirow{2}{*}{ Horn } & $d_{0}=1 \mathrm{~m}$ & \multirow{4}{*}{64.30} & 2.01 & 5.08 & 65.50 & 1.93 & 5.08 \\
\hline & & $d_{\text {opt }}=0.87 \mathrm{~m}$ & & 1.93 & 5.08 & & & \\
\hline & \multirow{2}{*}{ Omni } & $d_{0}=1 \mathrm{~m}$ & & 1.50 & 4.08 & 68.34 & 1.24 & 4.00 \\
\hline & & $d_{o p t}=0.47 \mathrm{~m}$ & & 1.24 & 4.00 & & & \\
\hline \multicolumn{9}{|l|}{ Models } \\
\hline \multicolumn{4}{|c|}{ 3GPP TR 38.901} & & 3.0 & & & \\
\hline \multicolumn{4}{|c|}{ 5GCM Office } & 1.73 & 3.02 & & & \\
\hline & \multicolumn{3}{|c|}{ 5GCM Shopping-Mall } & & 2.01 & & & \\
\hline & \multicolumn{3}{|c|}{ mmMAGIC (26 GHz) } & & & 62.32 & 1.38 & 1.18 \\
\hline & \multicolumn{3}{|c|}{ mmMAGIC (39 GHz) } & & & 65.94 & 1.38 & 1.18 \\
\hline
\end{tabular}

The optimized anchor distances $d_{\text {opt }}$ are in all cases below 1-m value, obtaining the closest value of $0.87 \mathrm{~m}$ with the Horn case at $26 \mathrm{GHz}$. These results confirm the convenience of using the 1-m anchor.

The 3GPP TR 38.901 and 5GCM models share the same PLE (1.73) of Table 6.1 and are only different in their $\sigma_{S F}$ 's. The best approximation is obtained for the Omni case at 26 $\mathrm{GHz}$ (1.70). Nevertheless, all the obtained PLEs (ranging from 1.50 to 2.01) are not so different from the value proposed in these models.

The mmMAGIC model provides an ABG-type path loss expression, but an FI-type one can be derived for a known frequency, as shown in Table 6.1. These FI coefficients are comparable with the obtained ones from the FI fitting (only $\beta$ for the Horn case at $26 \mathrm{GHz}$ deviates more). On the other hand, its $\sigma_{S F}$ is very low $(1.18 \mathrm{~dB})$, moderately far from the obtained (from 3.77 to $5.08 \mathrm{~dB}$ ). 
For graphical comparison purposes, Figure 6.4 shows the differences between the CI and FI fittings in each case. Altogether, the behavior holds for all cases: the FI fits better the measurements at smaller distances due to the floating-intercept coefficient.

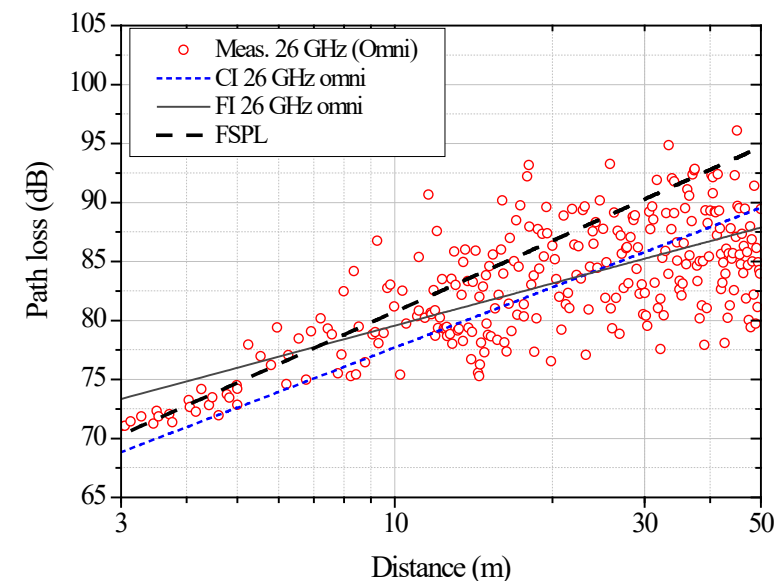

a)

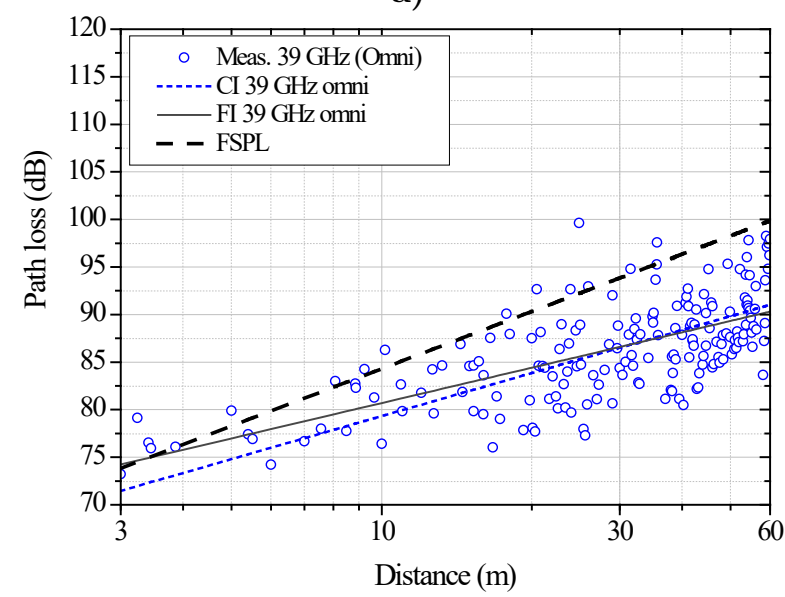

c)

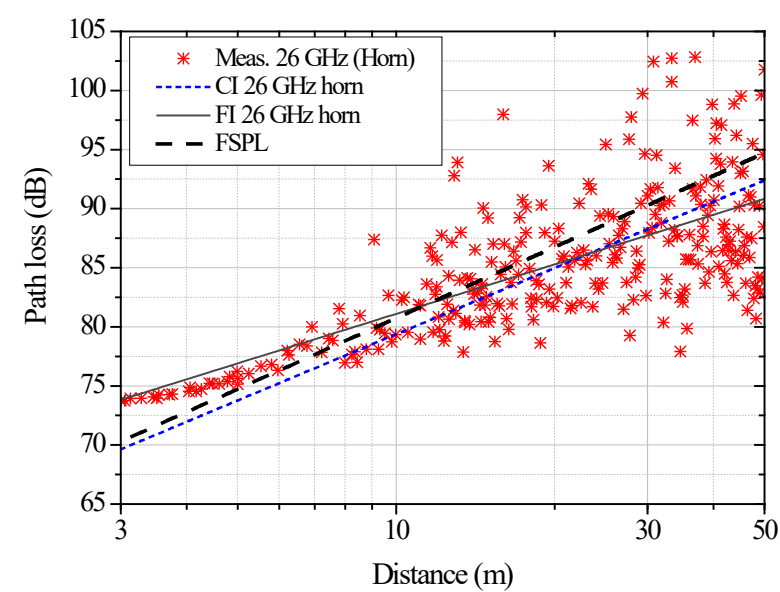

b)

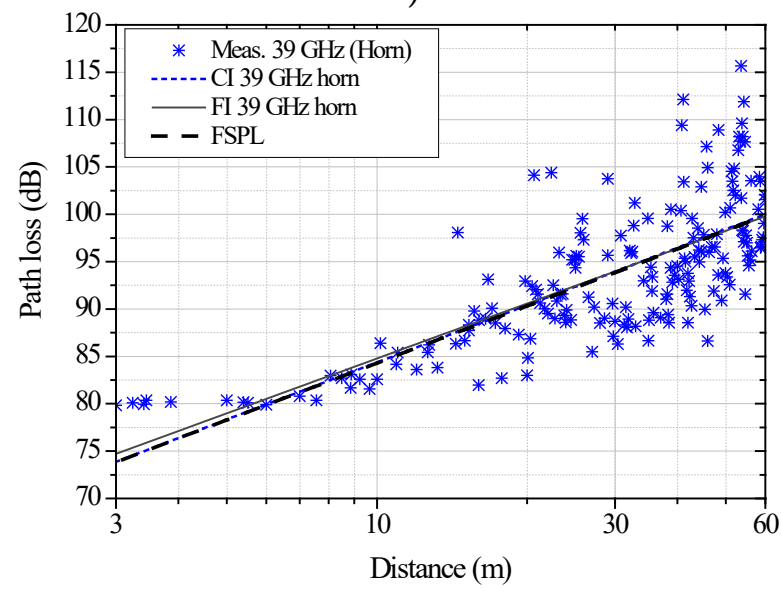

d)

Figure 6.4. Comparison between CI and FI LOS fittings. a) Omni and b) Horn cases at $26 \mathrm{GHz}$ and c) Omni and d) Horn cases at $39 \mathrm{GHz}$.

The comparison between the ABG fitting and mmMAGIC coefficients is shown in Table 6.2. Strictly speaking the ABG (which is presented as a multi-frequency fitting) is not carried out, since here the ABG-type expression is applied to only one frequency at a time (either 26 or $39 \mathrm{GHz}$ ), hence the fitting results revert to the FI fitting ones for each case. Nevertheless, for the sake of comparison with ABG-type models (as the mmMAGIC) the coefficients for the individual frequencies are provided. These coefficients must be taken with care because they only express a single-frequency fitting and several combinations of $\alpha$ and $\gamma$ parameters would provide exactly the same fitting results (here the model coefficients were set as initial values for applying the Levenberg-Marquardt algorithm, used to perform the least squares curve fitting). By doing so, the derived $\beta$ and $\gamma$ coefficients of Table 6.2 are comparable with their model counterparts, whereas the $\alpha$ coefficient remains unchanged. After testing several combinations, it was observed that the defined initial values modify the obtained $\alpha$ and $\gamma$, as expected, but have no influence in the behavior of $\beta$ (the PLE). 
Table 6.2. LOS ABG fitting and mmMAGIC coefficients

\begin{tabular}{cc|cccc}
\hline \hline \multirow{2}{*}{ Experiment } & \multicolumn{4}{|c}{ ABG coefficients } \\
& Antenna & $\boldsymbol{\alpha}$ & $\boldsymbol{\beta}$ & $\boldsymbol{\gamma}$ & $\boldsymbol{\sigma}_{\boldsymbol{S F}}(\mathbf{d B})$ \\
\hline $26 \mathrm{GHz}$ & Horn & 33.6 & 1.39 & 2.37 & 4.38 \\
& Omni & 33.6 & 1.19 & 2.41 & 3.77 \\
\hline \multirow{2}{*}{$39 \mathrm{GHz}$} & Horn & 33.6 & 1.93 & 2.00 & 5.08 \\
& Omni & 33.6 & 1.24 & 2.18 & 4.00 \\
\hline \multicolumn{2}{l|}{ Model } & 33.6 & 1.38 & 2.03 & 1.18 \\
\hline \multicolumn{2}{c}{ mmMAGIC Model }
\end{tabular}

The stochastic METIS model is a CI-type and its comparison is carried out separately in Table 6.3, since it is more restrictive than the result conditions, i.e. its frequency range is from 50 to $70 \mathrm{GHz}$ (this limitation is disregarded) and the distance can go only from 1.5 to $13.4 \mathrm{~m}$ (only measurements within this range are used). The approximations of the model coefficients with the derived ones are moderately good, especially for the $26 \mathrm{GHz}$ results.

Table 6.3. LOS FI fitting and METIS stochastic path loss model (from 1.5 to $13.4 \mathrm{~m}$ )

\begin{tabular}{lc|ccc}
\hline \hline Experiment & \multicolumn{3}{|c}{ FI coefficients } \\
& Antennas & $\boldsymbol{\alpha}$ & $\boldsymbol{\beta}$ & $\boldsymbol{\sigma}_{\boldsymbol{S F}}(\mathbf{d B})$ \\
\hline $26 \mathrm{GHz}$ & Horn & 63.64 & 1.79 & 2.58 \\
& Omni & 62.38 & 1.78 & 2.58 \\
\hline $39 \mathrm{GHz}$ & Horn & 74.79 & 0.86 & 1.22 \\
& Omni & 69.35 & 1.19 & 2.55 \\
\hline \multicolumn{3}{l}{ Model } & \multicolumn{3}{c}{} \\
\hline \multicolumn{2}{c|}{ METIS stochastic Model } & 68.8 & 1.84 & 2.0 \\
\hline \hline
\end{tabular}

The ITU-R Rec. P.1238-9 Model (based on the CI), does not provide coefficients for its entire frequency range (up to $100 \mathrm{GHz}$ ), but gives them for specific frequencies and scenarios. There are no coefficients for the frequencies at the scenario treated here (Corridor), but the model gives an $N$ coefficient with value of $19.5(N / 10=n=1.95)$ with a $\sigma_{S F}$ of $2.8 \mathrm{~dB}$ for 26 $\mathrm{GHz}$ in an Office environment (using omnidirectional antennas at both ends). These values may be compared with the $n$ of 1.70 and $\sigma_{S F}$ of $4.09 \mathrm{~dB}$ in Table 6.1 for the Omni case at that frequency. For $39 \mathrm{GHz}$, no value is given, but at $38 \mathrm{GHz}$ (the closest results to $39 \mathrm{GHz}$ ), $n$ 's of 2.03 and 1.86 and $\sigma_{S F}$ 's of 4.6 and $1.6 \mathrm{~dB}$ are provided for Office and Commercial scenarios respectively. In this case, the Horn configuration with an $n$ of 2.01 and $\sigma_{S F}$ of 5.08 dB approximates well the Office environment.

From the measurement fittings, the coefficients representing the path loss exponent, PLE (i.e. seen in Table 6.1 as $n$ for the CI and $\beta$ for the FI and ABG), are near or below 2 (FS condition); those for $39 \mathrm{GHz}$ with the METIS limits (shown in Table 6.3) being the more different ones. This PLE trend has been seen in almost all the consulted studies: corridors exhibit path loss lower than that in FS, with the typical distance power coefficient of around 1.8 [196] for CI-based equations. 
Also, a fitting with a dual-slope ABG model (for a single frequency) has been carried out to assess the improvement of using two slopes instead of one for the LOS case. For both frequencies, the dual-slope model with the Omni configuration does not give realistic results (i.e. it produces long break point distances compared with the maximum distance and/or a negative second slope). The Horn configuration obtains a moderate improvement for both frequencies, as Figure 6.5 shows. Different from the expected behavior, the second slope obtained at $26 \mathrm{GHz}$ is much lower than the first one.

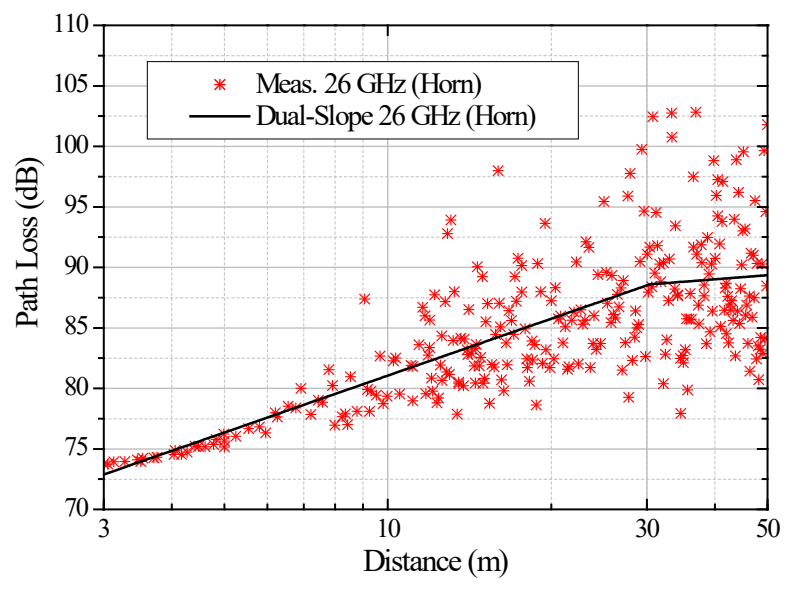

a)

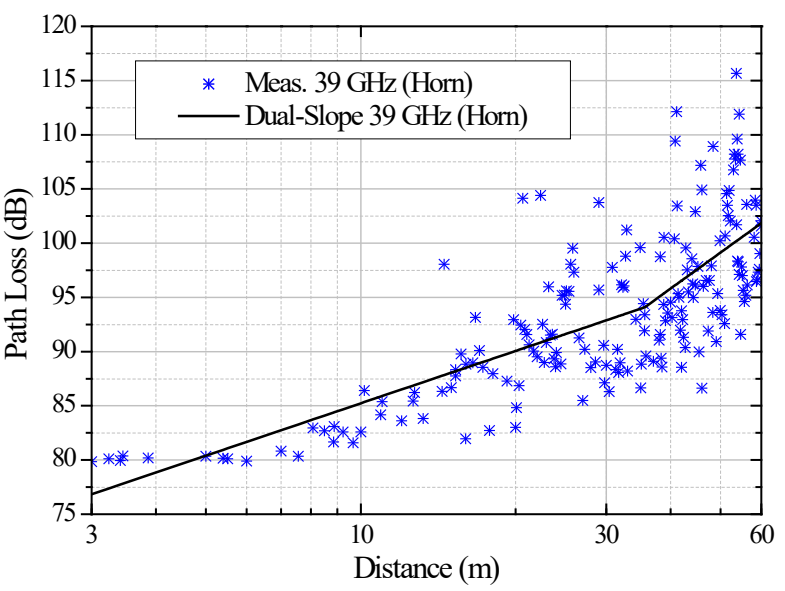

b)

Figure 6.5. ABG dual-slope fitting for the LOS Horn case at, a) $26 \mathrm{GHz}$ and b) $39 \mathrm{GHz}$.

The coefficients for the ABG dual-slope fitting are provided in Table 6.4.

Table 6.4. LOS coefficients for the dual-slope ABG (Horn case)

\begin{tabular}{l|cccccc}
\hline \hline & $\boldsymbol{\alpha}$ & $\boldsymbol{\beta}_{\mathbf{1}}$ & $\boldsymbol{\gamma}$ & $\boldsymbol{\beta}_{\mathbf{2}}$ & $\boldsymbol{d}_{\boldsymbol{B P}}$ & $\boldsymbol{\sigma}_{\boldsymbol{S F}}(\mathbf{d B})$ \\
\hline $\mathbf{2 6} \mathbf{G H z}$ & 0.32 & 1.60 & 4.57 & 0.15 & $30.77 \mathrm{~m}$ & 4.33 \\
$\mathbf{3 9} \mathbf{G H z}$ & 0.27 & 1.55 & 4.36 & 4.03 & $37.0 \mathrm{~m}$ & 4.94 \\
\hline \hline
\end{tabular}

From comparing the standard deviation of shadow fading shown in Table 6.4 with their counterparts of Table 6.1 (the Horn FI cases), it is seen than the improvement is small (e.g., $4.38 \mathrm{~dB}$ the FI against $4.33 \mathrm{~dB}$ the dual-slope $\mathrm{ABG}$ at $26 \mathrm{GHz}$ ), then the use of the dualslope ABG model may not be worth the associated increase in model complexity.

\section{Shadow fading modeling}

The shadow fading, displayed in Figures 6.6 and 6.7 for $26 \mathrm{GHz}$ and $39 \mathrm{GHz}$ respectively, is another important parameter of the measurements. It is usually evaluated by means of its standard deviation $\sigma_{S F}(\mathrm{~dB})$ (its mean should be close to $0 \mathrm{~dB}$ ) and can be obtained by subtracting the respective fitting curve from the measurements.

The shadow fading for the FI and ABG (single frequency) are equal (both fittings return the same statistics, e.g. the same respective values of $\sigma_{S F}(\mathrm{~dB})$ in Tables 6.1 and 6.2) and are displayed in Figures $6.6 \mathrm{~b}$ and $6.7 \mathrm{~b}$. 


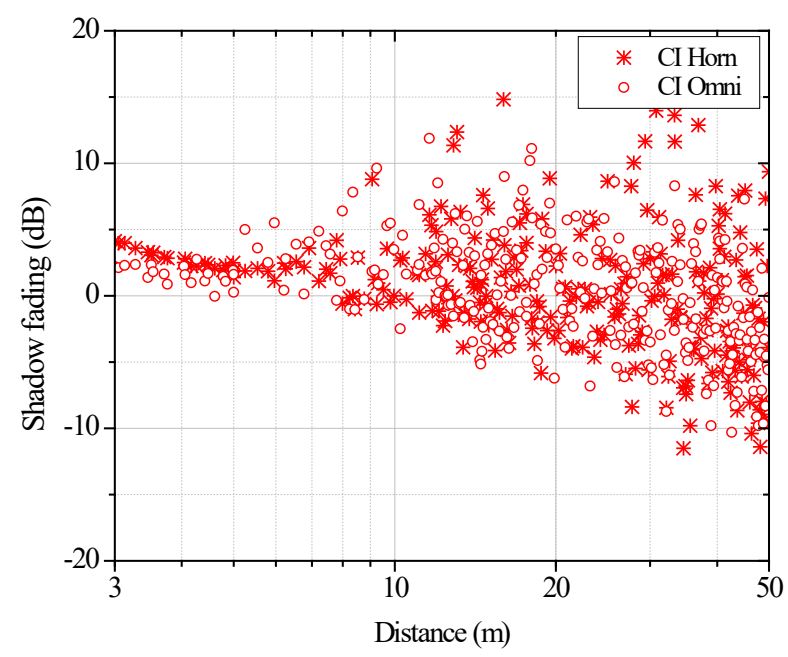

a)

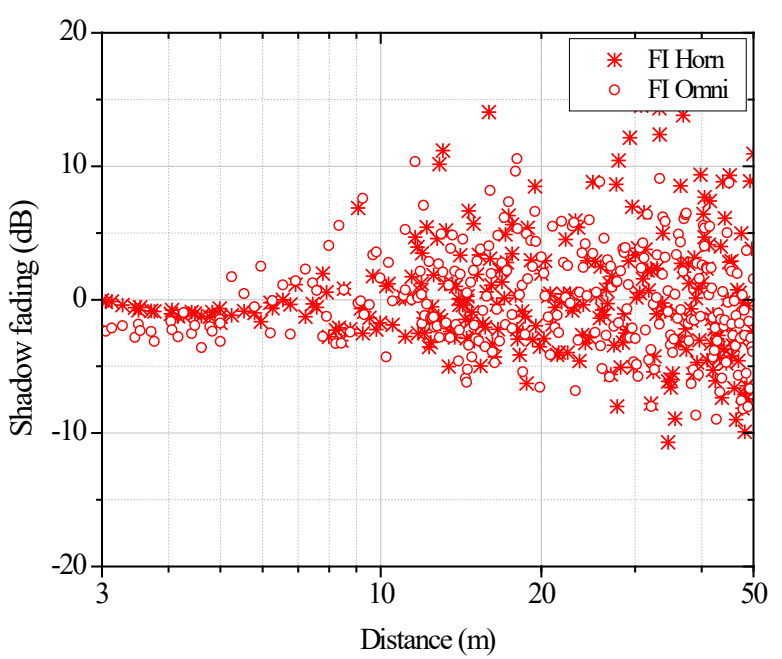

b)

Figure 6.6. LOS shadow fading for $26 \mathrm{GHz}$, a) CI fitting and b) FI fitting.

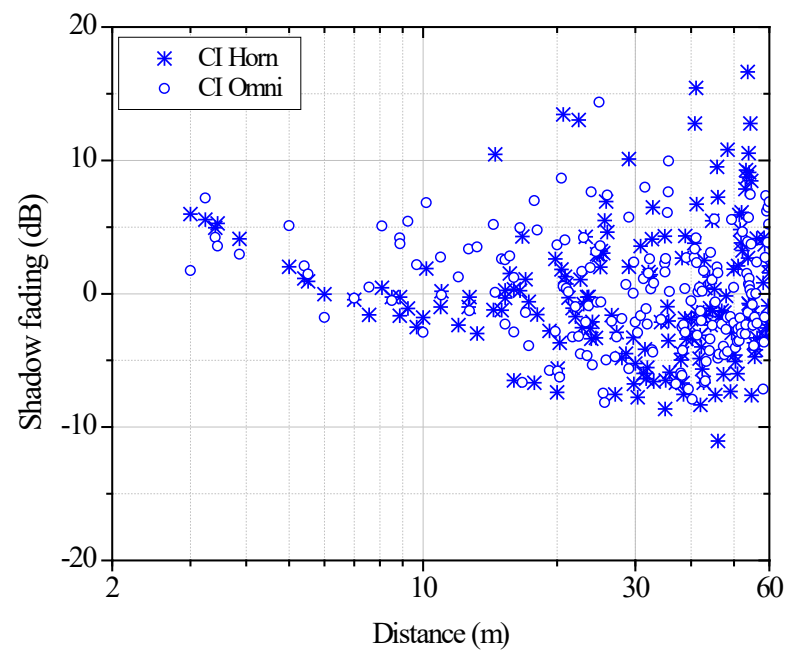

a)

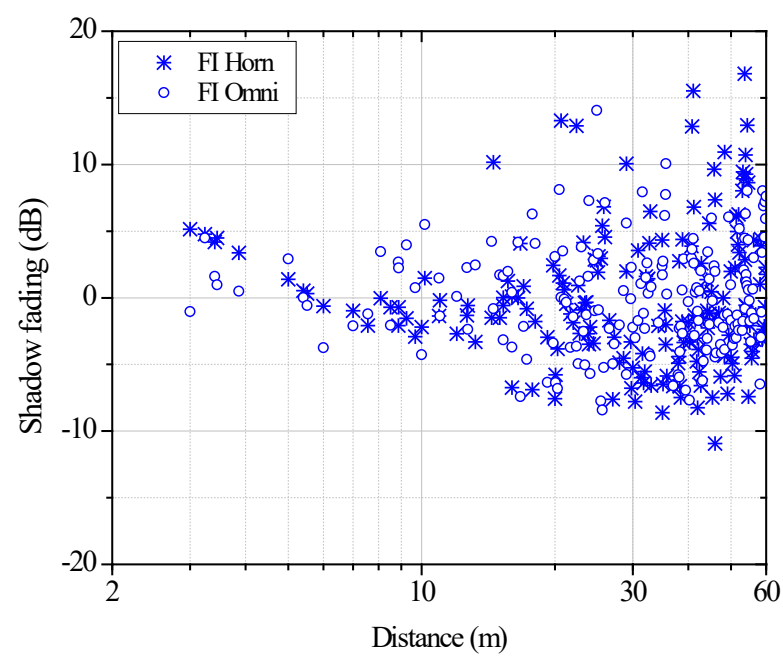

b)

Figure 6.7. LOS shadow fading for $39 \mathrm{GHz}$, a) CI fitting and b) FI fitting.

Also, shadow fading is usually represented by a Gaussian distribution. This representation can be assessed from the respective histogram and CDF analysis for each case. In Figure 6.8, the shadow fading histograms are represented for $26 \mathrm{GHz}$. The Gaussian distributions with the mean and standard deviation of the respective histogram are drawn in red line (Modeled) and seem to fit well the measurements. 


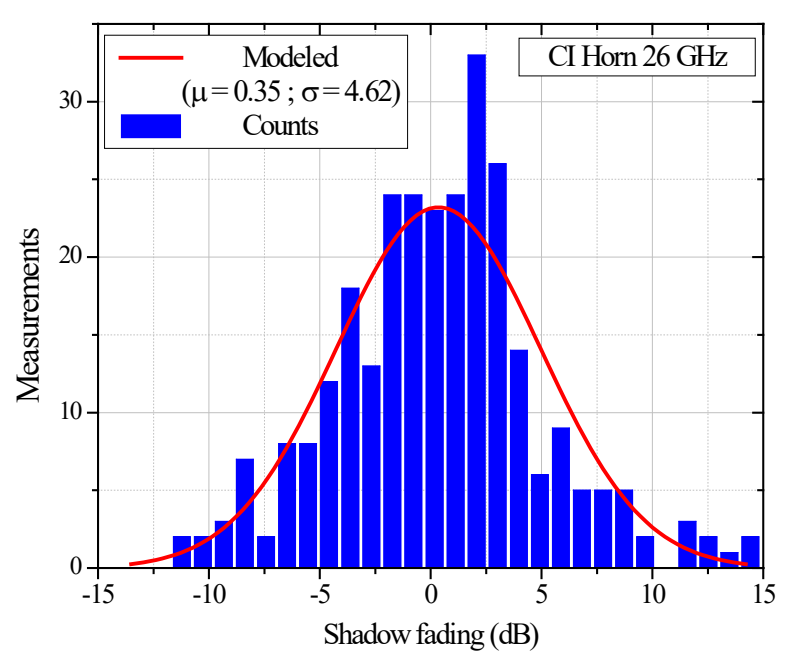

a)

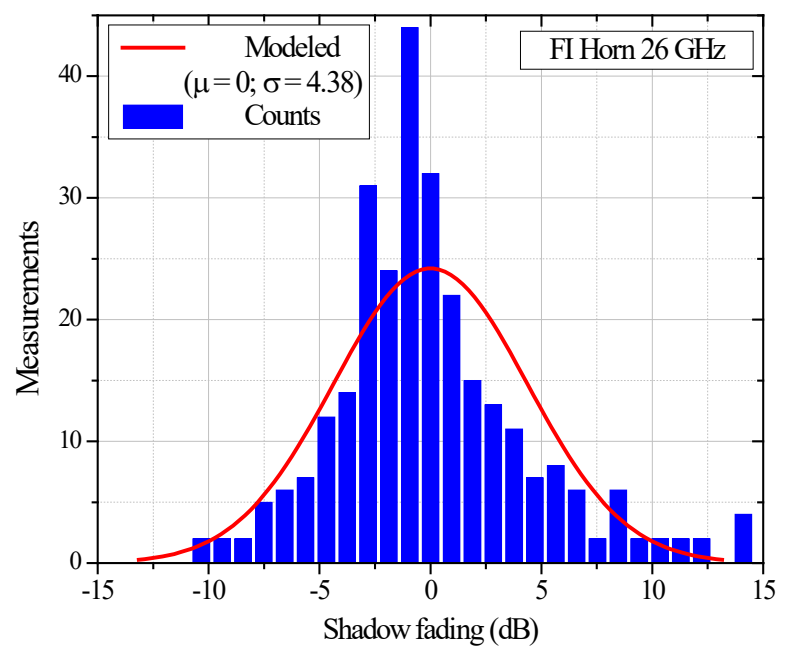

c)

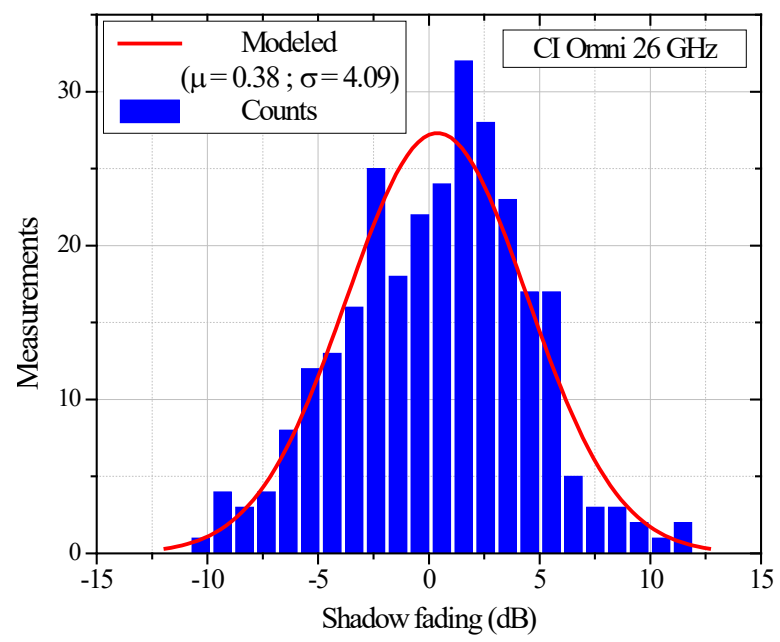

b)

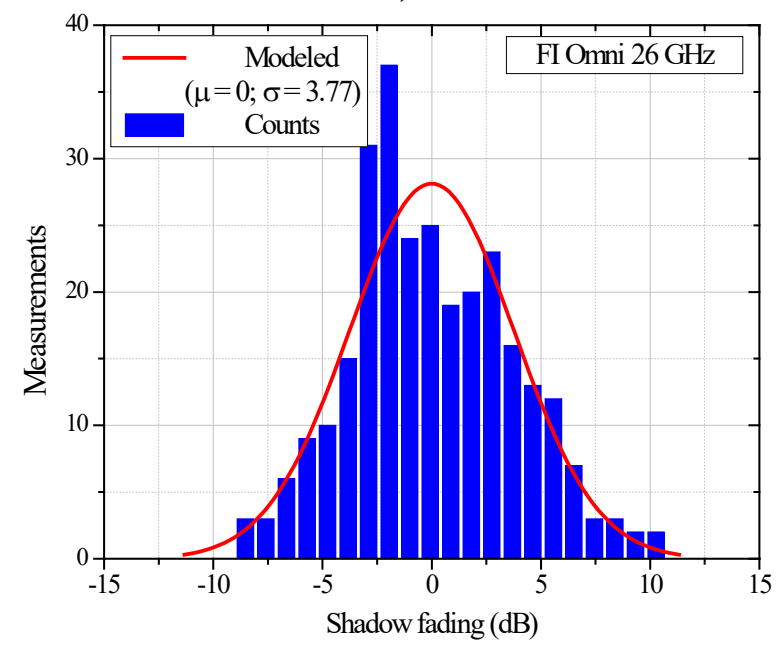

d)

Figure 6.8. $26 \mathrm{GHz}$ LOS shadow fading histograms and modeled Gaussian distributions for fittings and configurations a) CI, Horn, b) CI, Omni, c) FI, Horn and d) FI, Omni.

Regarding the CDF, a very good estimate is seen in Figure 6.9 between the empirical CDF of the shadow fading (Experimental in blue color) and the Gaussian CDF (Modeled in red color). 


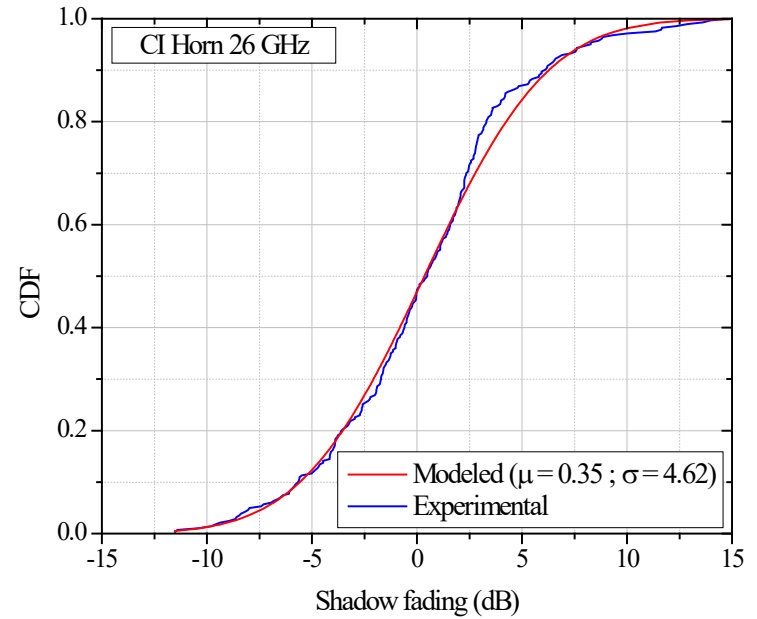

a)

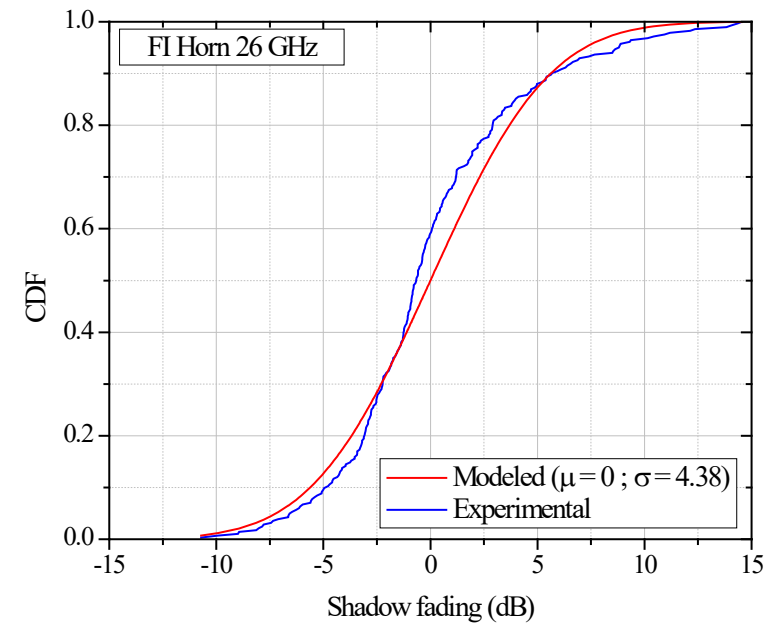

c)

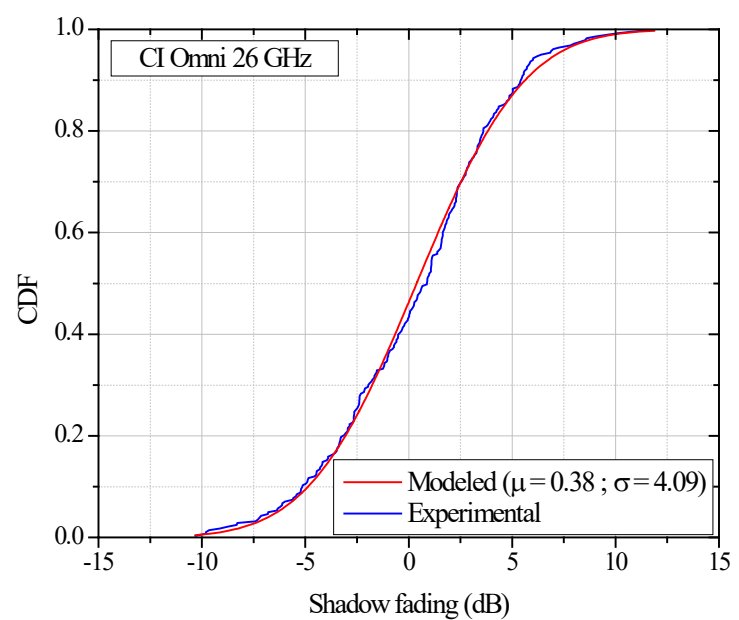

b)

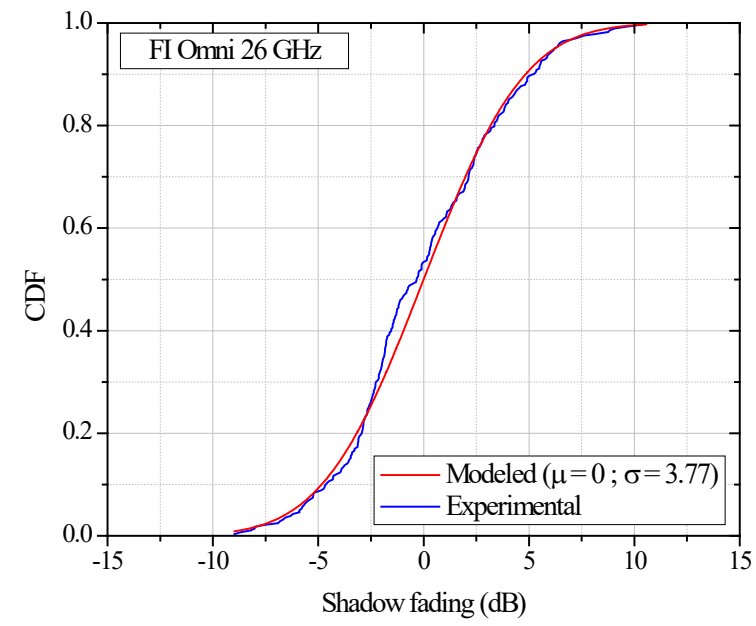

d)

Figure 6.9. $26 \mathrm{GHz}$ LOS shadow fading CDF distributions for fittings and configurations a) CI,Horn, b) CI, Omni, c) FI, Horn and d) FI, Omni.

Figure 6.10 is the analogous of Figure 6.8 for the $39 \mathrm{GHz}$ case: it shows the shadow fading histograms and the Gaussian distributions modeled with the mean and standard deviation of each of them. Again, the histograms are well fitted by the Gaussian distributions. 


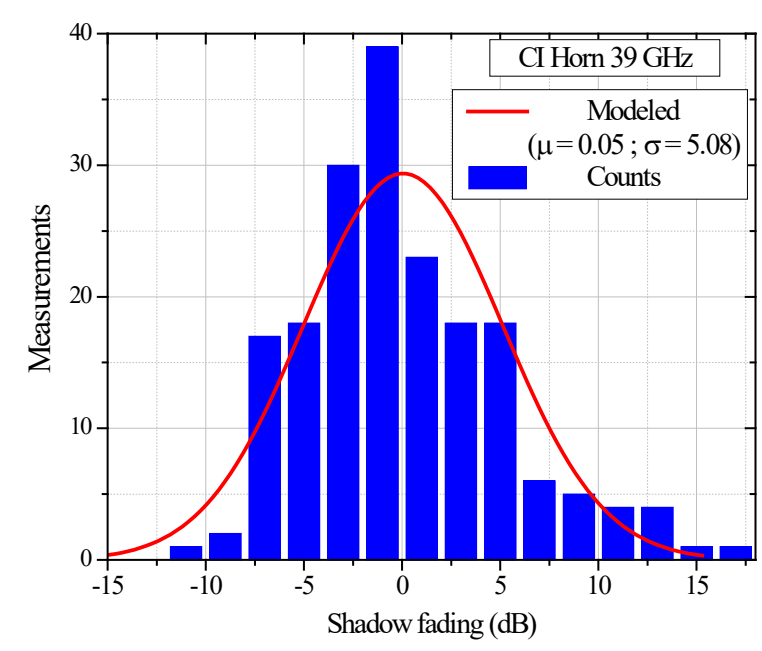

a)

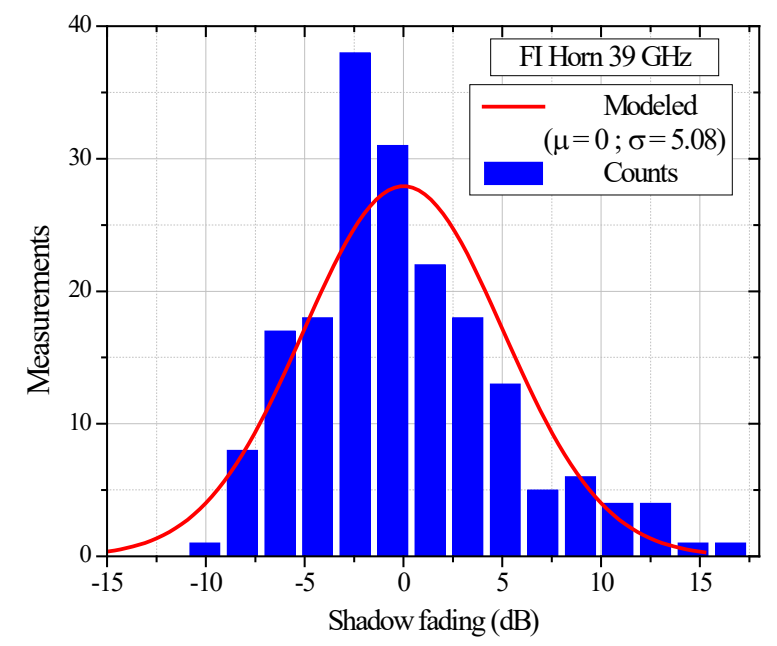

c)

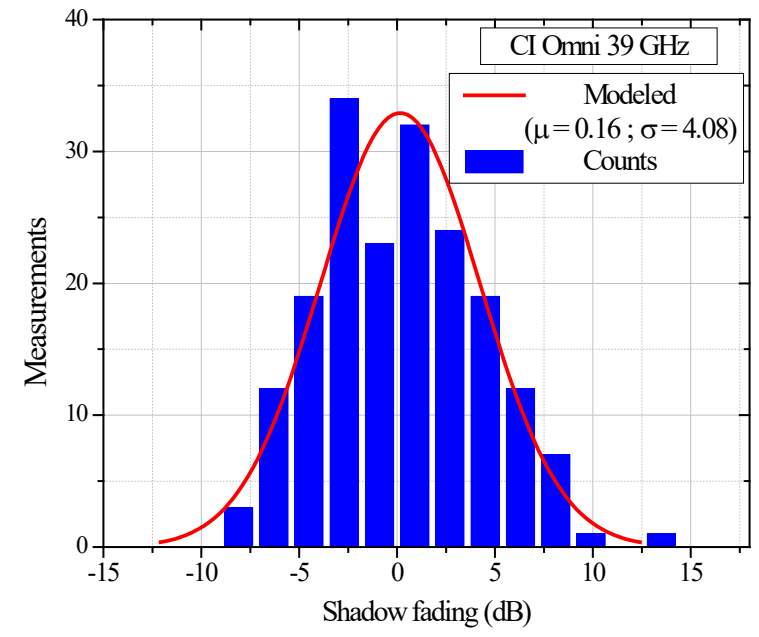

b)

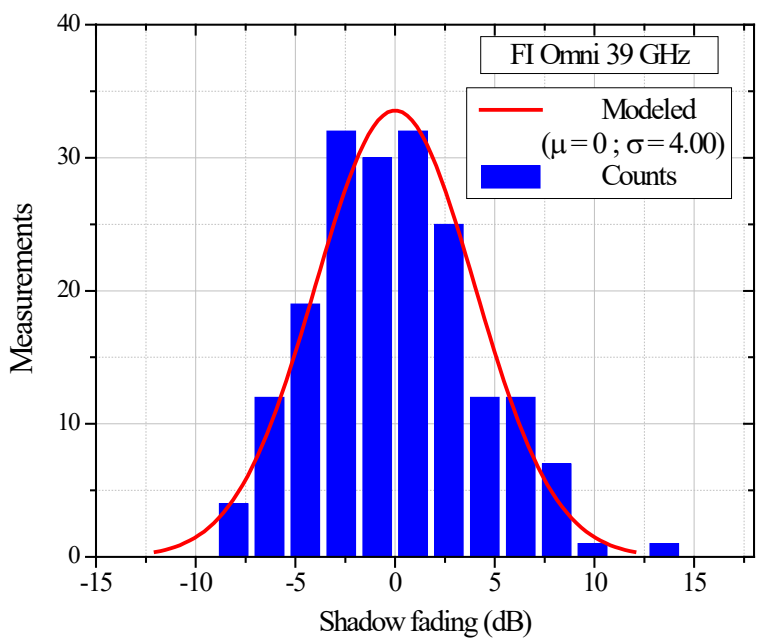

d)

Figure 6.10. $39 \mathrm{GHz}$ LOS shadow fading histograms and modeled Gaussian distributions for fittings and configurations a) CI, Horn, b) CI, Omni, c) FI, Horn and d) FI, Omni.

The CDF for the $39 \mathrm{GHz}$ case, both the measured and the modeled ones, are represented in Figure 6.11. The approximations are very good, with higher differences for shadow fading values close to $0 \mathrm{~dB}$. 


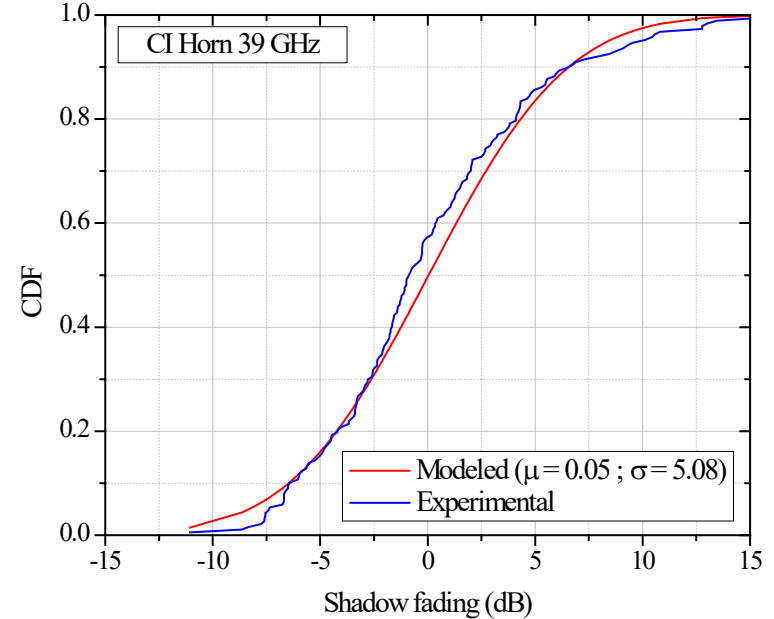

a)

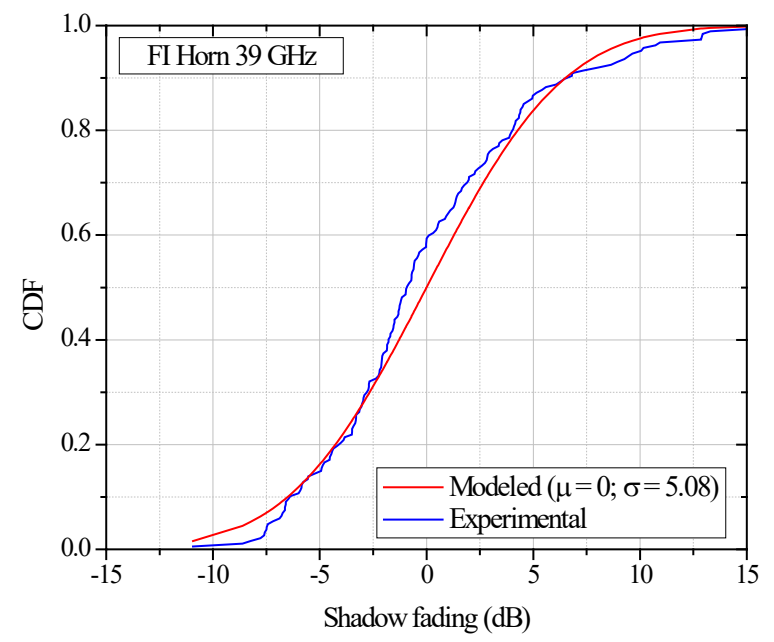

c)

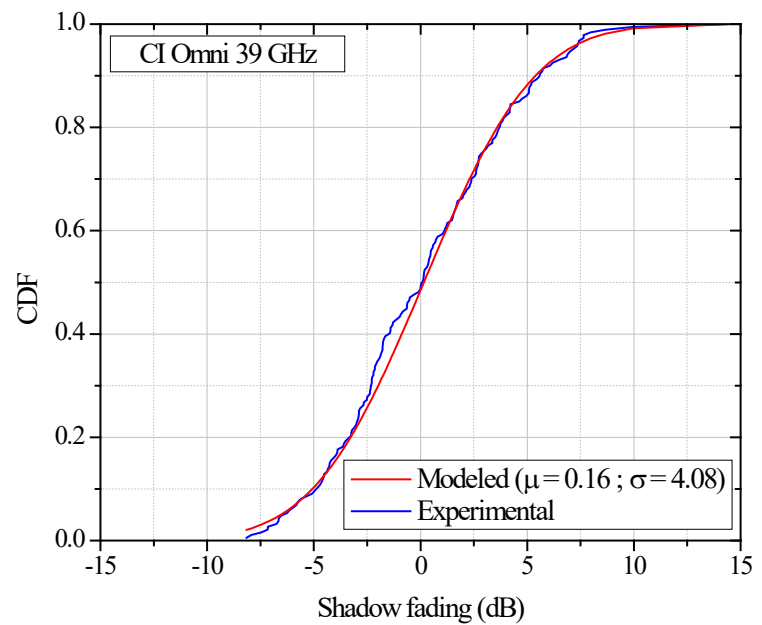

b)

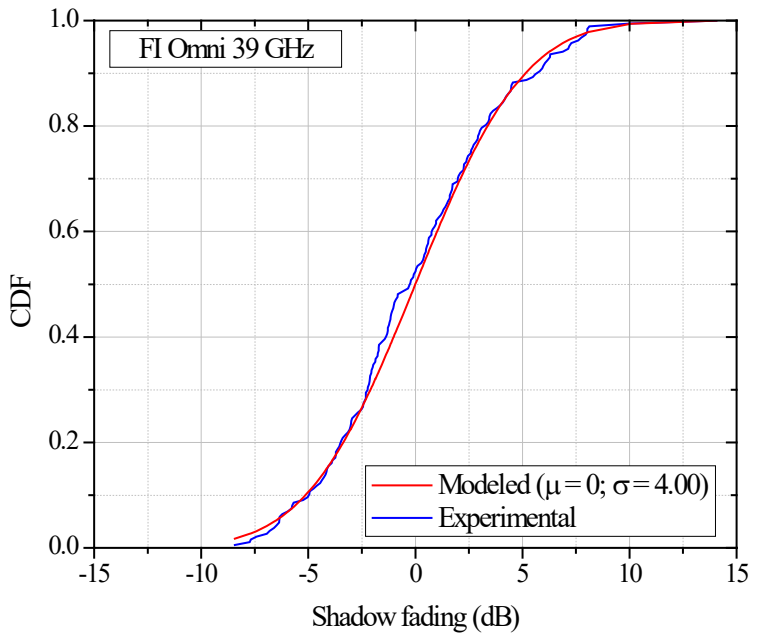

d)

Figure 6.11. 39 GHz LOS shadow fading CDF distributions for fittings and configurations a) CI, Horn, b) CI, Omni, c) FI, Horn and d) FI, Omni.

The mean and standard deviation for each estimated Gaussian distribution are collected in Table 6.5.

Table 6.5. Statistical values of the shadow fading results for the Indoor LOS measurements

\begin{tabular}{ccccc}
\hline \hline Experiment & Configuration & Fitting & Mean & Std. \\
\hline $\mathbf{2 6} \mathbf{~ G H z}$ & Horn & CI & 0.35 & 4.62 \\
& & FI & 0.0 & 4.38 \\
& \multirow{2}{*}{ Omni } & CI & 0.38 & 4.09 \\
& & FI & 0.0 & 3.77 \\
\hline $\mathbf{3 9} \mathbf{G H z}$ & \multirow{2}{*}{ Horn } & CI & 0.05 & 5.08 \\
& & FI & 0.0 & 5.08 \\
& \multirow{2}{*}{ Omni } & CI & 0.16 & 4.08 \\
& & FI & 0.0 & 4.00 \\
\hline \hline
\end{tabular}

Overall, the FI fittings represent better a zero-mean Gaussian distribution since the CI mean values are higher than the FI ones, as Table 6.5 shows. The larger CI deviations are 
due to the FSPL 1-m anchor, which produces a higher deviation at shorter distances (as was shown in Figure 6.4). It is particularly interesting the case of $39 \mathrm{GHz}$ for the Horn configuration, since the CI and FI fittings give similar standard deviation and the lowest mean for the CI cases is obtained. Also, the respective CI and FI approximations are closer for the $39 \mathrm{GHz}$ case than for the $26 \mathrm{GHz}$ one.

\subsubsection{NLOS results}

The NLOS measurements have been gathered in three independent passes. They were taken with the transmitter unit in Corridor $A$ and with the receiver unit in Corridor $B$ (see Figure 6.1). The receiver unit is separated at approximately 3,5 and $21 \mathrm{~m}$ in the first (Pass 1), the second (Pass 2) and the third pass (Pass 3) respectively from point $C$ in Figure 6.1. Then, the distance from $C$ to the transmitter unit can be known if needed, once the total distance is also known. Here, it is worth to point out that the gain of IF amplifier was set to $36 \mathrm{~dB}$ for obtaining a stronger received signal. This value was also removed in the calibration. Measurements at 26 and $39 \mathrm{GHz}$ are taken for the same positions.

Table 6.6 collects the information regarding each pass. Since the total number of measurements is not large enough to allow an independent analysis of the three passes, the results are represented together in Figures 6.12 and 6.13 for 26 and $39 \mathrm{GHz}$ respectively. Only a few measurements were taken at short distances (less than $10 \mathrm{~m}$ ) since real NLOS scenarios should take place at longer distances. Now, the point clouds for the Horn and Omni measurements are in general more separated than in the LOS case.

Table 6.6. Information regarding NLOS measurements

\begin{tabular}{lccc}
\hline \hline & Pass 1 & Pass 2 & Pass 3 \\
\hline Distance from RX to point C & 3 & 5 & 21 \\
Number of measurements & 17 & 25 & 19 \\
Total distance range (m) & $34.65-66.65$ & $6.65-54.65$ & $22.65-58.65$ \\
Amplification (dB) & & $36 \mathrm{~dB}$ & \\
\hline \hline
\end{tabular}

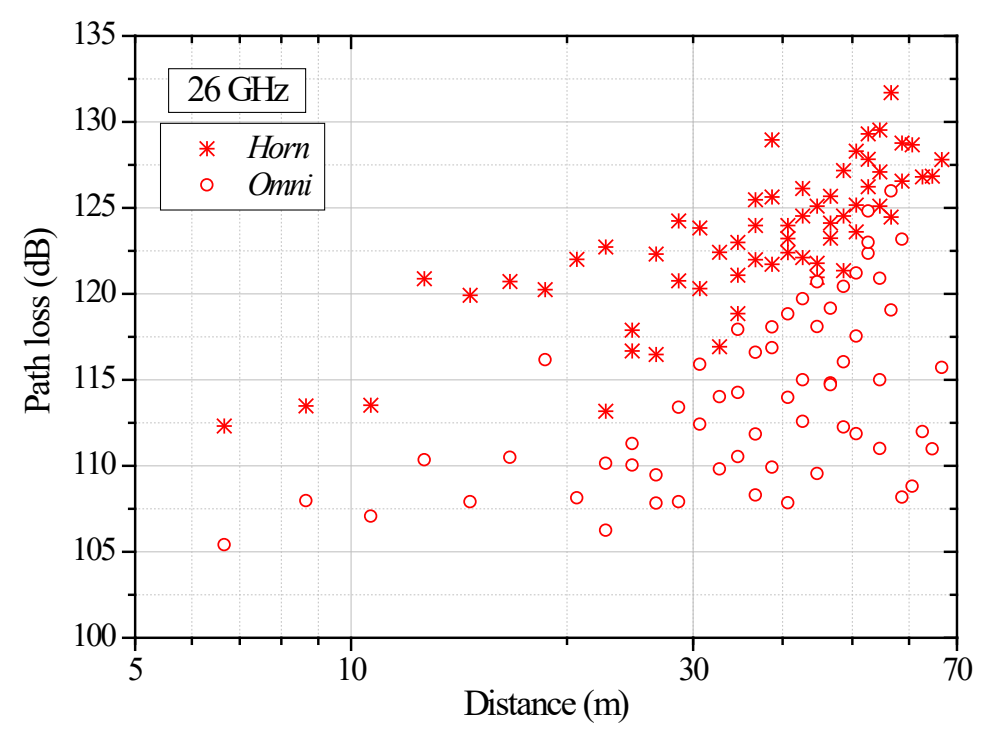

Figure 6.12. NLOS measurements at $26 \mathrm{GHz}$. 


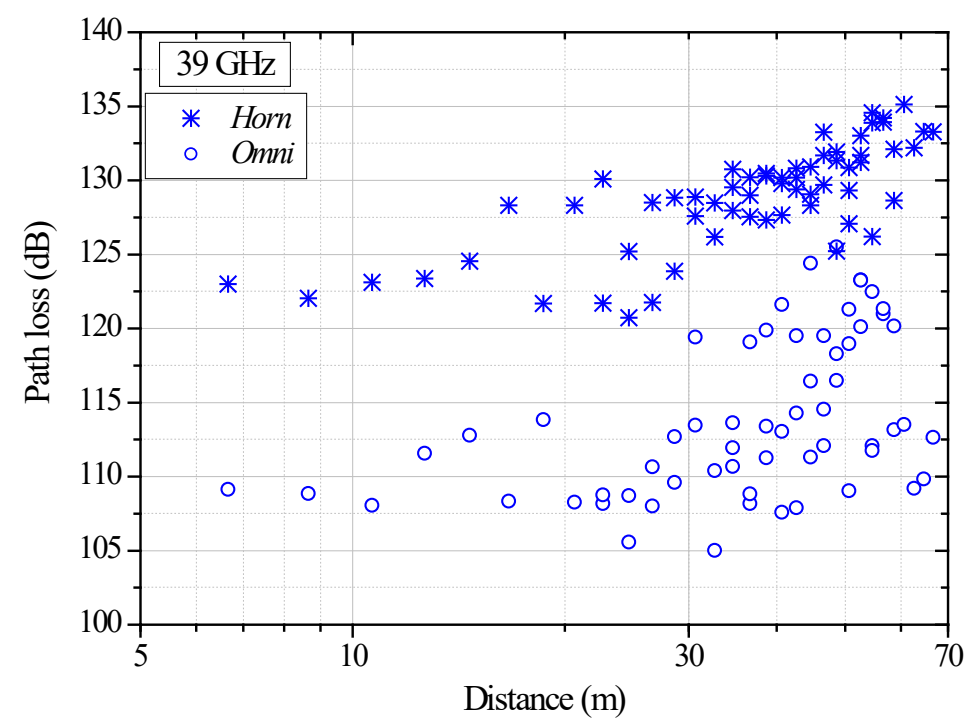

Figure 6.13. NLOS measurements at $39 \mathrm{GHz}$.

\subsubsection{NLOS modeling}

\section{NLOS path loss modeling}

For modeling, the CI, FI and ABG (for single frequency) fittings are used. It can be seen that almost all models use two or more coefficients to describe the NLOS condition. Only the 3GPP TR 38.901 for Indoor-Office provides a CI-based option.

Table 6.7 shows the comparison between the CI and FI obtained coefficients and those provided by the models. The 1-m anchor is used in the CI fitting.

Table 6.7. Comparison of coefficient between the CI and FI fittings with NLOS models

\begin{tabular}{lc|cc|ccc}
\hline \hline Experiments & & \multicolumn{2}{|c|}{ CI } & \multicolumn{3}{c}{ FI } \\
& Antenna & $\boldsymbol{n}$ & $\boldsymbol{\sigma}_{\boldsymbol{S F}}(\mathbf{d B})$ & $\boldsymbol{\alpha}$ & $\boldsymbol{\beta}$ & $\boldsymbol{\sigma}_{\boldsymbol{S F}}(\mathbf{d B})$ \\
\hline $26 \mathrm{GHz}$ & Horn & 3.98 & 5.83 & 98.92 & 1.56 & 2.58 \\
& Omni & 3.39 & 6.20 & 93.65 & 1.31 & 4.26 \\
\hline $39 \mathrm{GHz}$ & Horn & 4.11 & 6.65 & 109.59 & 1.24 & 2.39 \\
& Omni & 3.16 & 6.42 & 96.01 & 1.15 & 4.72 \\
\hline Models & 3.19 & & & \\
\hline 3GPP TR.38.901 & & & & & \\
METIS & & & & & \\
\hline
\end{tabular}

It is seen now that the PLE of the 3GPP NLOS model (3.19) is higher than for the FS condition (i.e. 2), a behavior also seen in the fittings. In all cases, the model $\sigma_{S F}$ gives a value higher than the obtained ones.

The METIS NLOS model has been compared with all the measurements, disregarding its limitation in distance, due to the fact that there are very few measurements in its defined distance range (from 4 to $16.1 \mathrm{~m}$ ). It gives a $\beta$ of 3.59 relatively higher than the derived ones 
(ranging from 1.15 to 1.56 ) and an $\alpha$ of 94.3 that remains within the obtained range (from 93.01 to 109.59$)$.

The ITU-R model of Rec. ITU-R P.1238-9 is a CI-type, but neither for $26 \mathrm{GHz}$ nor 39 $\mathrm{GHz}$ it provides an $n$ value in NLOS conditions. Using the values provided for the closest frequencies, the model gives for Office and Commercial environments at $28 \mathrm{GHz} n$ 's of 2.99 and 2.76 and $\sigma_{S F}$ 's of $6.6 \mathrm{~dB}$ and $6.4 \mathrm{~dB}$ respectively. At $38 \mathrm{GHz}, n$ 's of 2.96 and 2.59 and $\sigma_{S F}$ 's of $6.8 \mathrm{~dB}$ and $5.5 \mathrm{~dB}$ are given for the same scenarios. For all cases, higher PLEs compared with the model ones have been obtained and the $\sigma_{S F}$ 's of the fittings are in the same range as their model counterparts.

For a single frequency, the ABG fittings return the same results as the FI, this is seen if the $\sigma_{S F}$ and $\beta$ values of FI and ABG of Tables 6.7 and 6.8 are compared (they are equal). However, the ABG coefficients are collected and presented separately for each frequency since several models provide an ABG-type expression for NLOS. The ABG single-frequency comparison with models is seen in Table 6.8. Again, initial values close to the model coefficients were set for applying the Levenberg-Marquardt algorithm (the same reasoning presented in sub-section 6.1.2 is also used here).

Table 6.8. Comparison of the coefficients of the ABG fitting and NLOS models

\begin{tabular}{|c|c|c|c|c|c|}
\hline \multirow[t]{2}{*}{ Experiments } & \multirow[b]{2}{*}{ Antennas } & \multicolumn{4}{|c|}{ ABG coefficients } \\
\hline & & $\alpha$ & $\beta$ & $\gamma$ & $\sigma_{S F}(\mathrm{~dB})$ \\
\hline \multirow[t]{2}{*}{$26 \mathrm{GHz}$} & Horn & 23.53 & 1.56 & 5.33 & 2.58 \\
\hline & Omni & 23.51 & 1.31 & 4.96 & 4.26 \\
\hline \multirow[t]{2}{*}{$39 \mathrm{GHz}$} & Horn & 16.37 & 1.24 & 5.85 & 2.39 \\
\hline & Omni & 16.31 & 1.15 & 5.00 & 4.72 \\
\hline \multicolumn{6}{|l|}{ Models } \\
\hline \multirow{2}{*}{\multicolumn{2}{|c|}{ 5GCM Office / 3GPP TR.38.901 }} & 17.30 & 3.83 & 2.49 & 8.03 \\
\hline & & 18.09 & 3.21 & 2.24 & 6.97 \\
\hline \multicolumn{2}{|c|}{ mmMAGIC } & 15.2 & 3.69 & 2.68 & 8.03 \\
\hline
\end{tabular}

The derived $\alpha$ values remain close to the model counterparts. More noticeable differences arise for $\beta$ and $\gamma$. Also, the $\sigma_{S F}$ 's predicted by the models are higher than the experimental ones.

The 5GCM NLOS model also provides a CIF-type expression for Office and ShoppingMall scenarios, but no comparison are done in these cases due to the lack of a higher number of frequencies more consecutively spaced (the CIF is a multi-frequency fitting) which does not allow a proper derivation of the $f_{0}$ parameter of (3.7) presented in sub-section 3.2.1. However, if the CIF fitting is used for the single frequencies, the result reverts to the CI model for each case.

Figure 6.14 shows the CI and FI /ABG NLOS fittings at 26 and $39 \mathrm{GHz}$ for the Horn and Omni configuration. The differences between the fitted curves are now more pronounced than for the LOS case. This behavior is produced by the FSPL-term in the CI fitting, which is much lower than the analogous FI term in NLOS condition. 


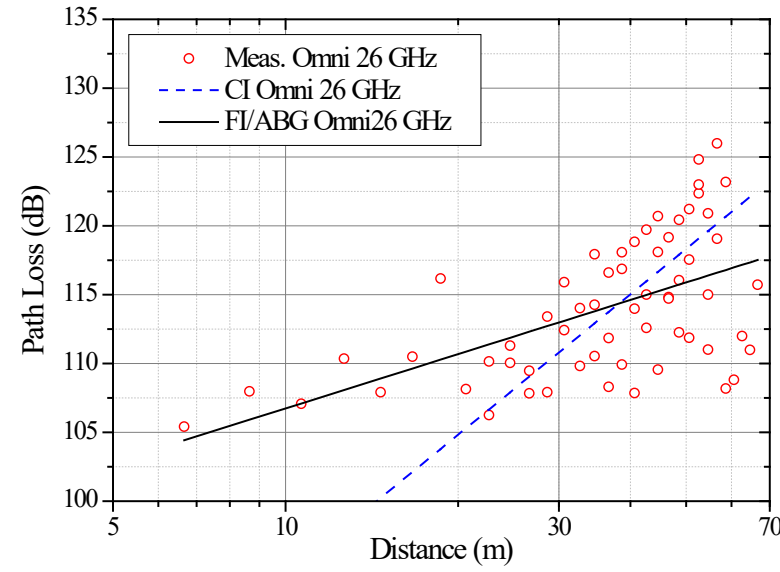

a)

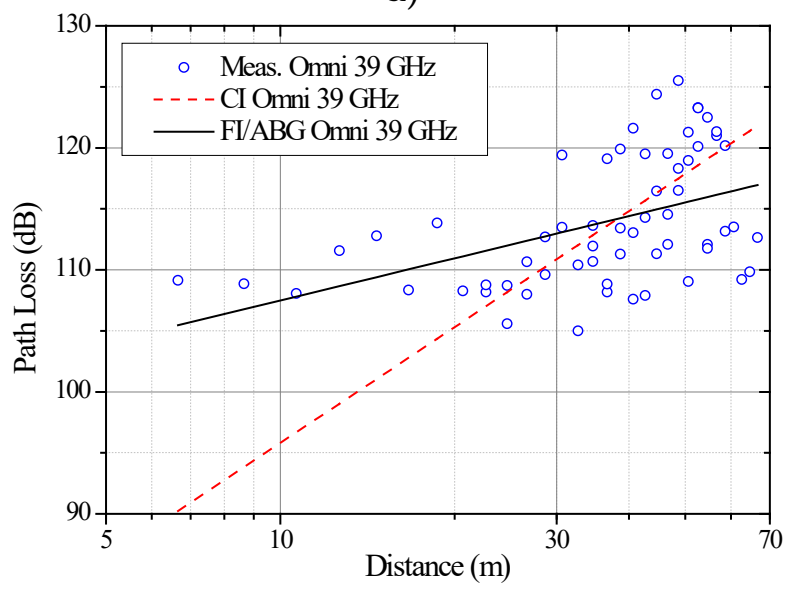

c)

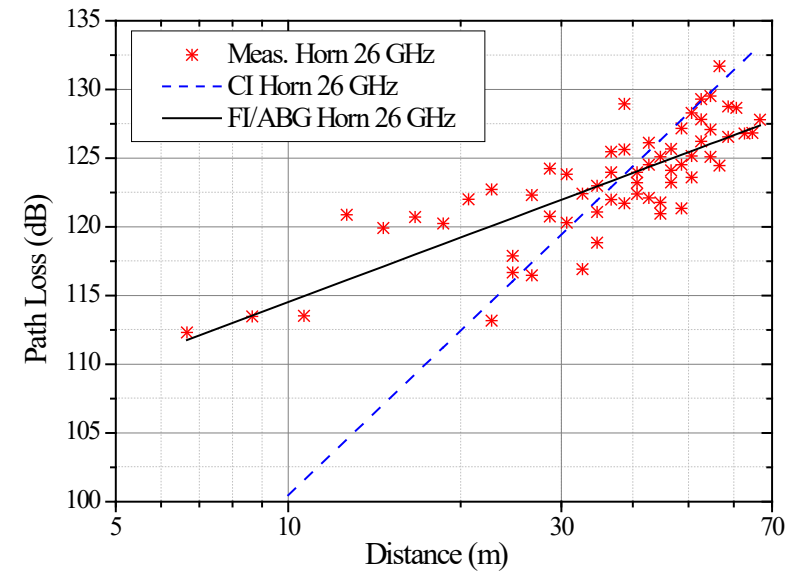

b)

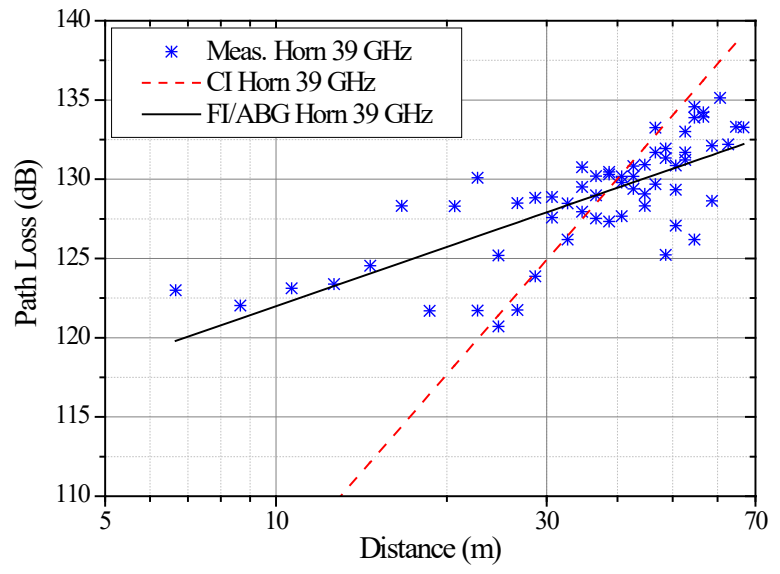

d)

Figure 6.14. Comparison between CI and FI/ABG NLOS fittings. $26 \mathrm{GHz}$ measurements a) Omni and b) Horn cases and $39 \mathrm{GHz}$ measurements c) Omni and d) Horn cases.

As was presented in section 4.2 and 4.3, some models use a dual-slope expression for modeling the NLOS results. Applying the dual-slope ABG fitting some improvements were obtained for the Horn case, although they are very small. The fittings can be seen graphically in Figure 6.15. As for the LOS condition, no improvements were obtained from using a dualslope expression for the Omni case at both frequencies.

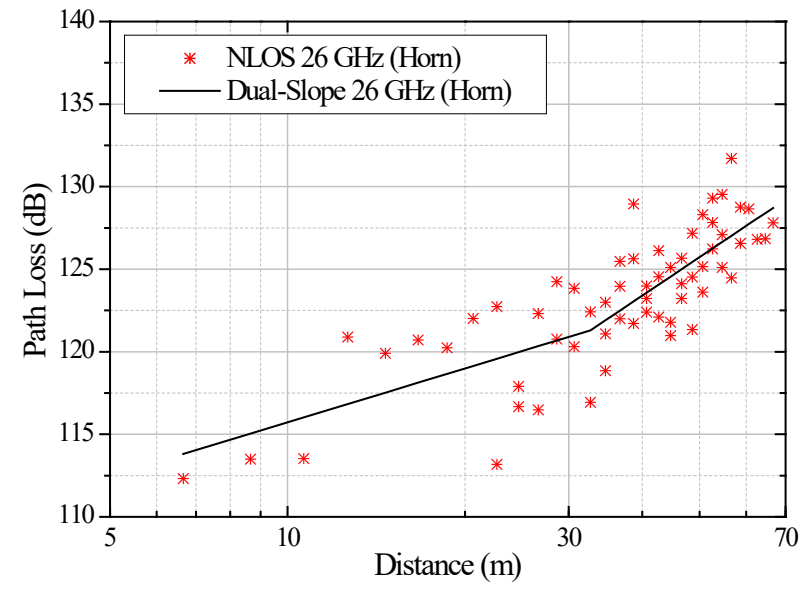

a)

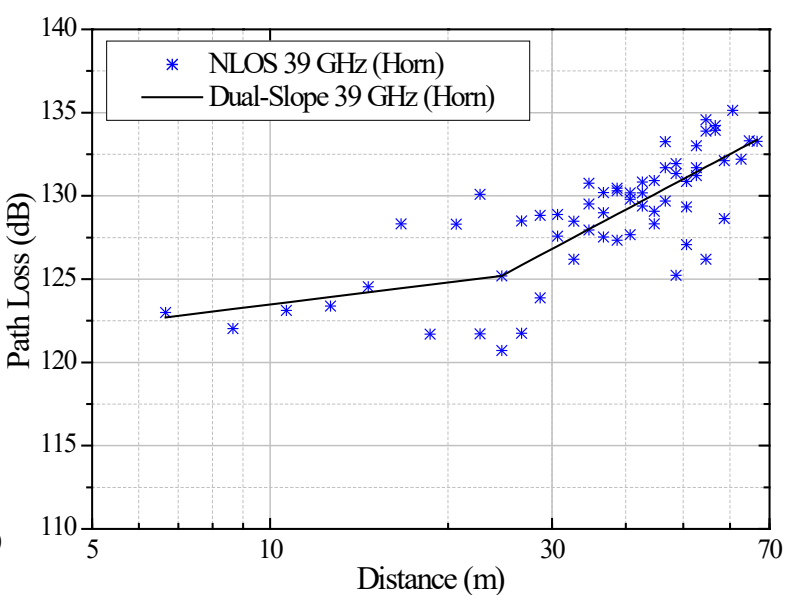

b)

Figure 6.15. ABG dual-slope fitting for the NLOS Horn case at, a) $26 \mathrm{GHz}$ and b) $39 \mathrm{GHz}$. 
From comparing the $\sigma_{S F}$ 's of the dual-slope ABG fitting of Table 6.9 with their counterparts of Table 6.8, only a small improvement in their favor is seen (e.g. $2.48 \mathrm{~dB}$ against $2.58 \mathrm{~dB}$ for the $26 \mathrm{GHz}$ case). Table 6.9 also shows the coefficients and standard deviations of the 5GCM NLOS models for comparison purpose.

Table 6.9. NLOS coefficients for the dual-slope ABG (Horn case) compared with the coefficients of the 5GCM

\begin{tabular}{l|cccccc}
\hline \hline Experiments & \multicolumn{7}{c}{ ABG dual-slope coefficients } \\
& $\boldsymbol{\alpha}$ & $\boldsymbol{\beta}_{\mathbf{1}}$ & $\gamma$ & $\boldsymbol{\beta}_{\mathbf{2}}$ & $\boldsymbol{d}_{\boldsymbol{B P}}$ & $\boldsymbol{\sigma}_{\boldsymbol{S F}}(\mathbf{d B})$ \\
\hline $\mathbf{2 6} \mathbf{~ G H z}$ & 22.42 & 1.08 & 5.82 & 2.39 & 32.65 & 2.48 \\
$\mathbf{3 9} \mathbf{~ G H z}$ & 22.41 & 0.44 & 6.07 & 1.89 & 24.65 & 2.26 \\
\hline Models & 33.00 & 1.70 & 2.49 & 4.17 & 6.90 & 7.78 \\
\hline 5GCM Office & 22.17 & 2.90 & 2.24 & 11.47 & 147.0 & 6.36 \\
\hline 5GCM Shopping-Mall &
\end{tabular}

The 5GCM Shopping-Mall has a break point distance much longer than the maximum measured distance. On the other hand, the 5GCM Office has a relatively short break point distance compared with the obtained ones. The fitting results give lower PLEs and $\sigma_{S F}$ 's compared with the models.

\section{Shadow fading modeling}

The NLOS shadow fading values extracted from using the FI and CI fittings are seen in Figures 6.16 and 6.17 for 26 and $39 \mathrm{GHz}$ respectively. Here, the FI and ABG fittings share the same shadow fading.

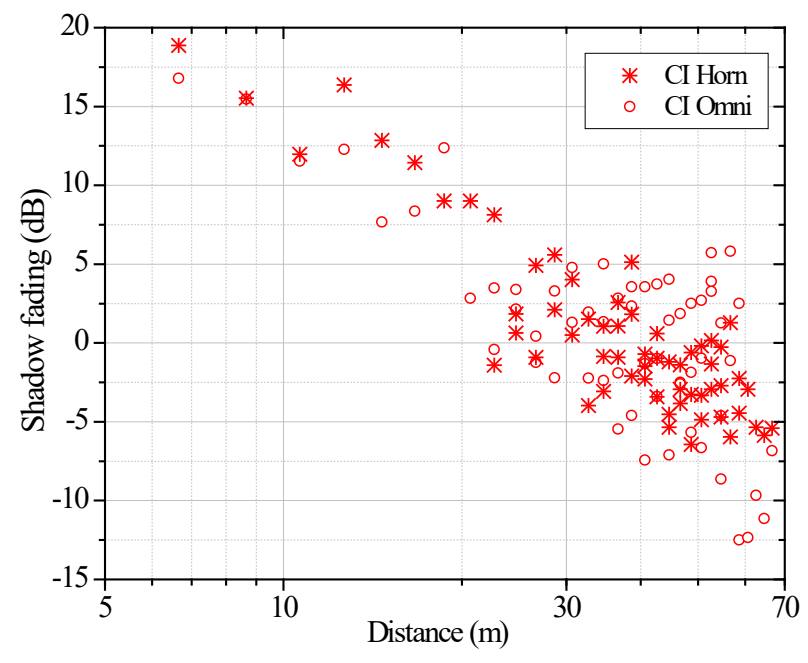

a)

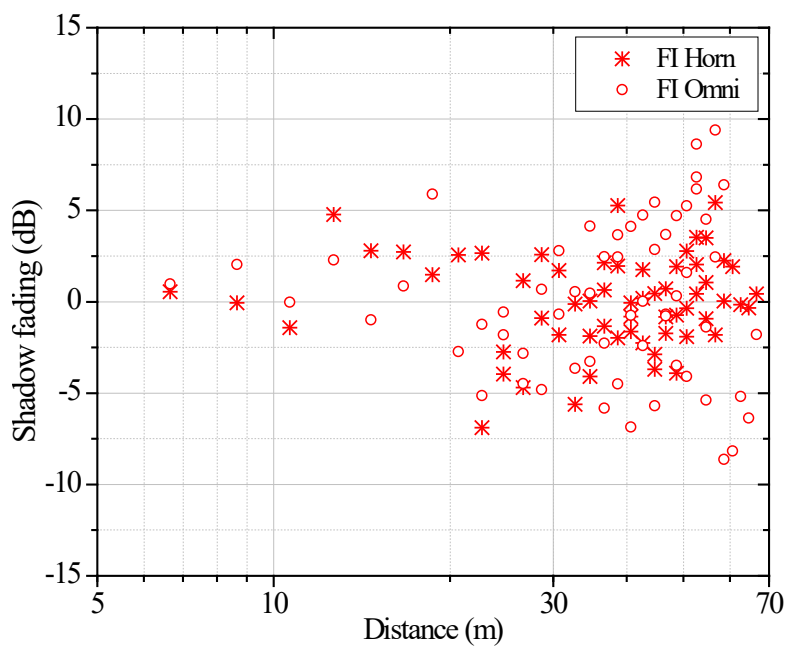

b)

Figure 6.16. NLOS shadow fading for $26 \mathrm{GHz}$, a) CI fitting and b) FI/ABG fitting. 


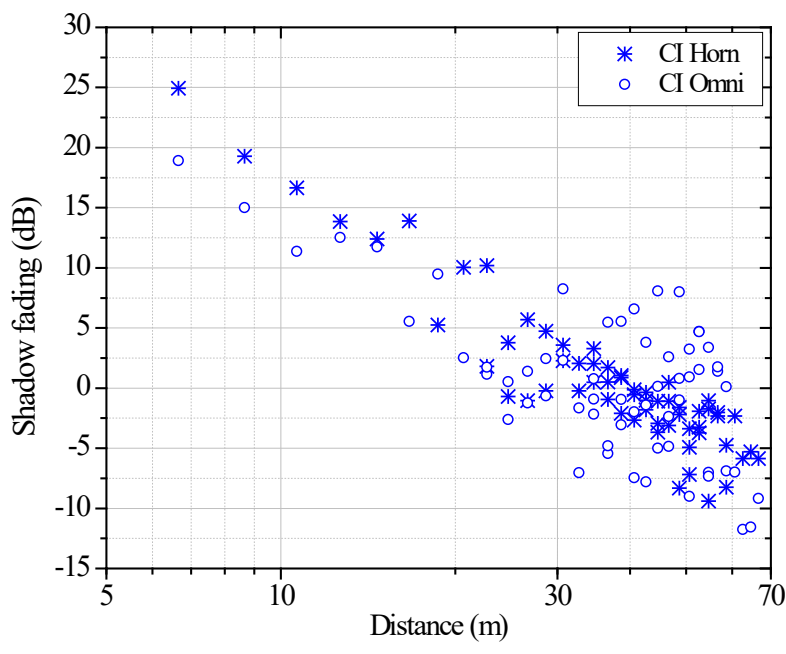

a)

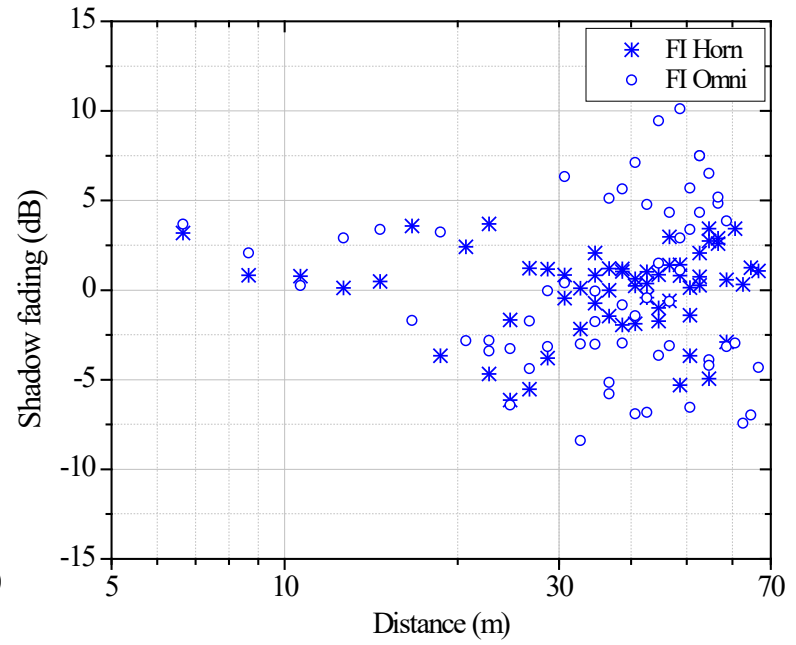

b)

Figure 6.17. NLOS shadow fading for $39 \mathrm{GHz}$, a) CI and b) FI/ABG fittings.

The highest deviation for shorter distances is seen again for the CI fitting in Figures 6.16 and 6.17 , being more prominent than for the LOS case.

The Gaussian distributions modeled with the resulting mean and standard deviation of the respective shadow fading are seen in Figure 6.18 for the $26 \mathrm{GHz}$ case. In this case, the Gaussian distribution fits well the results, although there is now a lower bin number compared with the LOS results due to the smaller amount of measurements. 


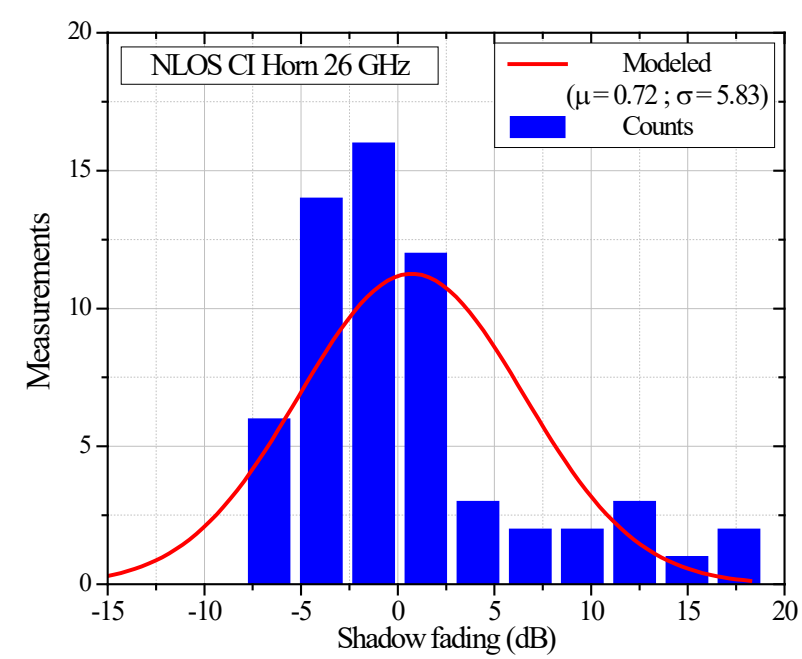

a)

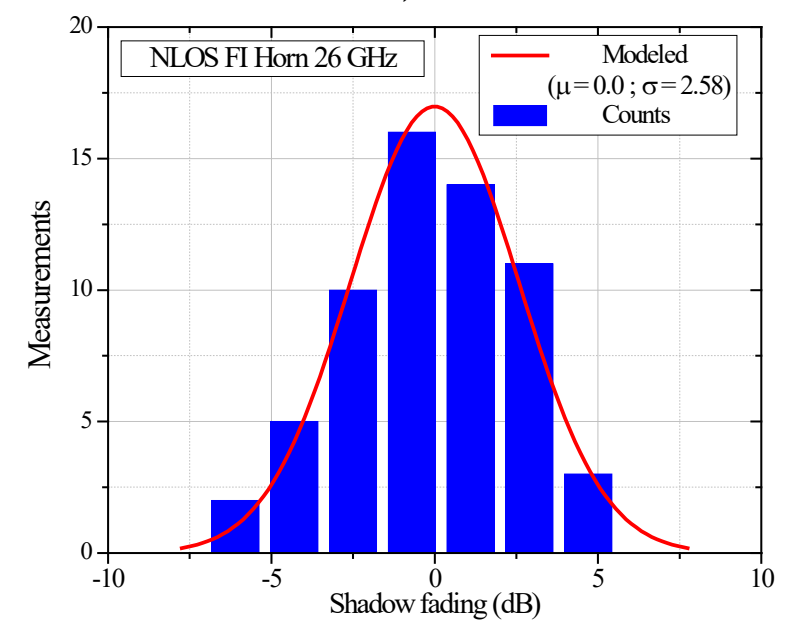

c)

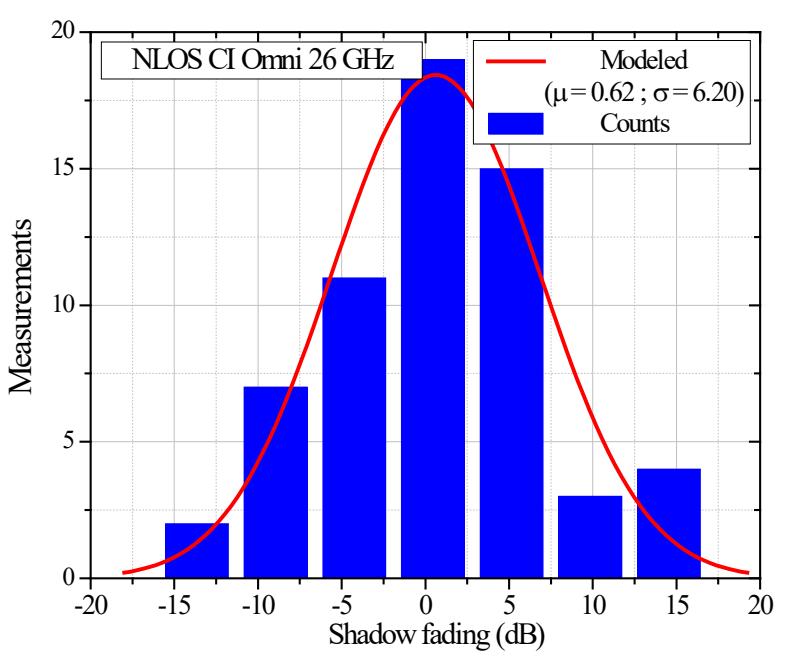

b)

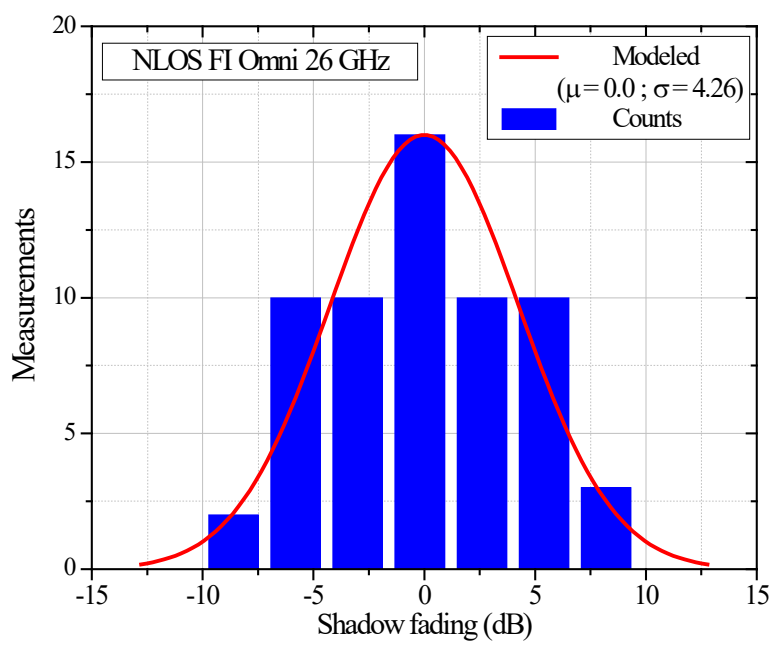

d)

Figure 6.18. $26 \mathrm{GHz}$ NLOS shadow fading histograms and modeled Gaussian distributions for fittings and configurations a) CI, Horn, b) CI, Omni, c) FI, Horn and d) FI, Omni.

The comparisons of the derived CDF Gaussian distributions and the experimental ones are shown in Figure 6.19, showing a reasonable agreement. 


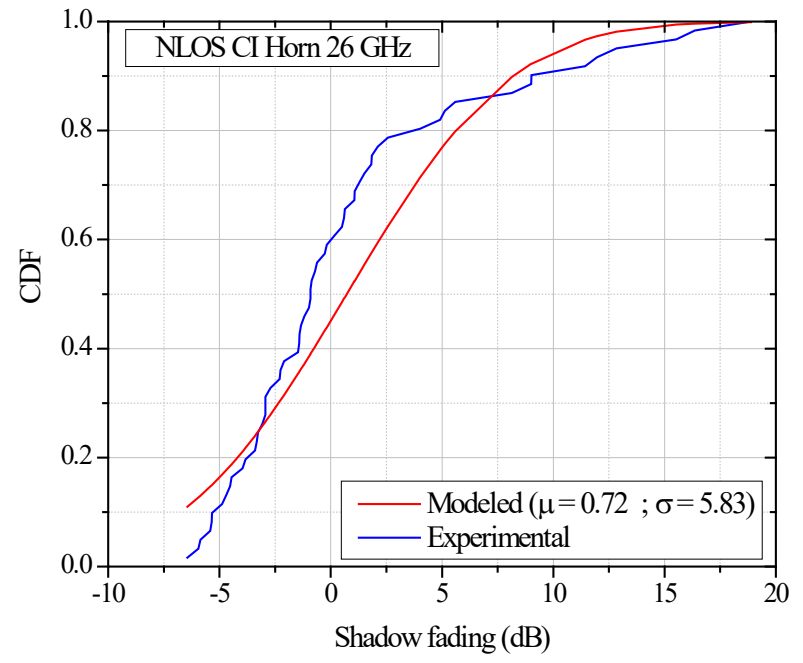

a)

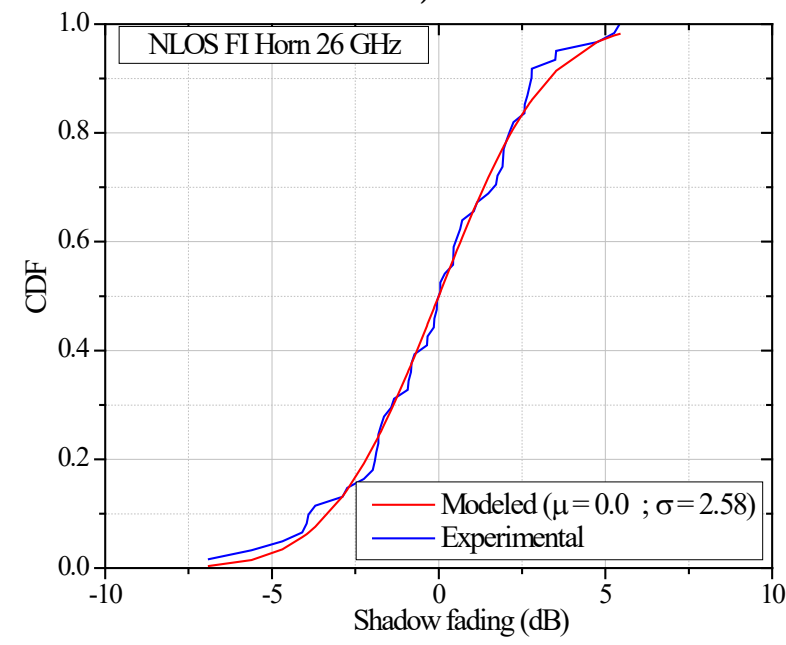

c)

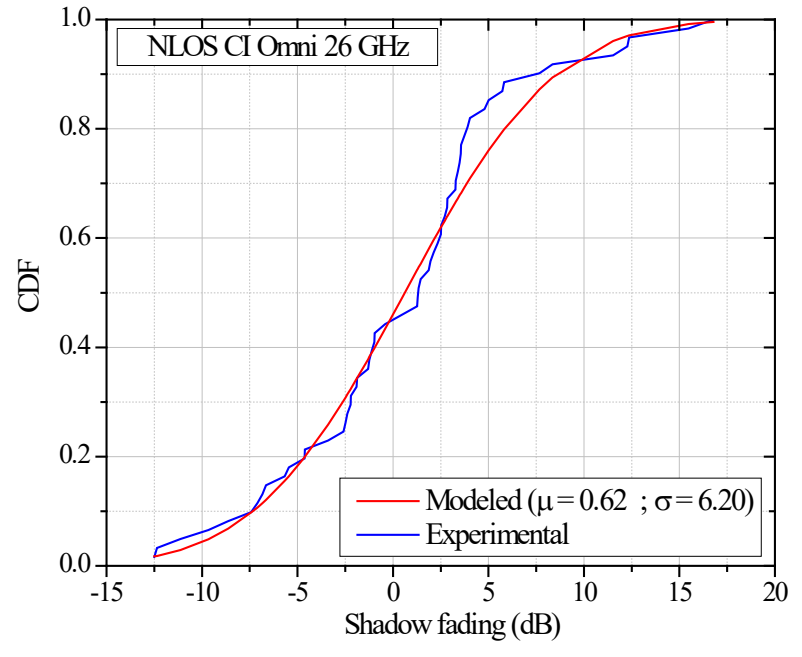

b)

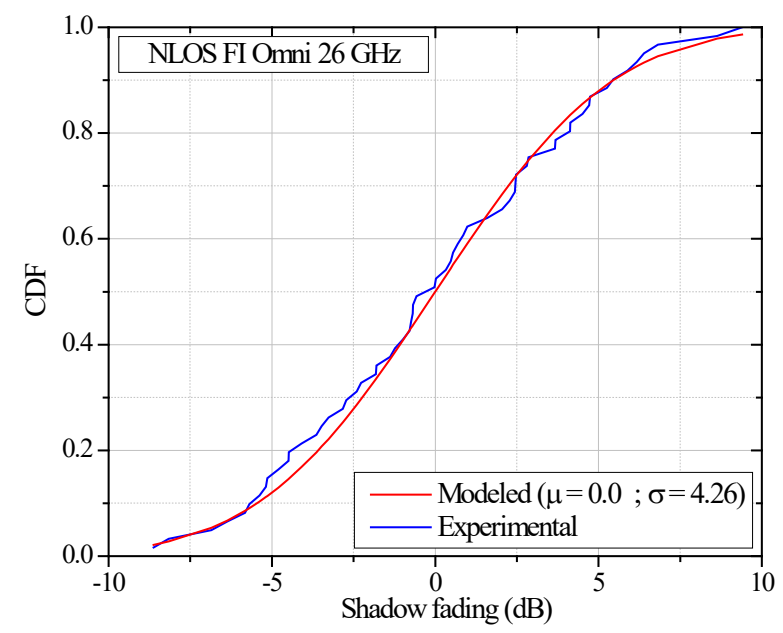

d)

Figure 6.19. $26 \mathrm{GHz}$ NLOS CDF distributions for fittings and configurations a) CI, Horn, b) CI, Omni, c) FI, Horn and d) FI, Omni.

Now for the $39 \mathrm{GHz}$ case, analogous results are derived and presented in Figures 6.20 and 6.21. For this case, the Gaussian distribution does not fit well the histogram in most of the cases; this is also seen in the differences between the experimental CDFs and the Gaussian ones. 


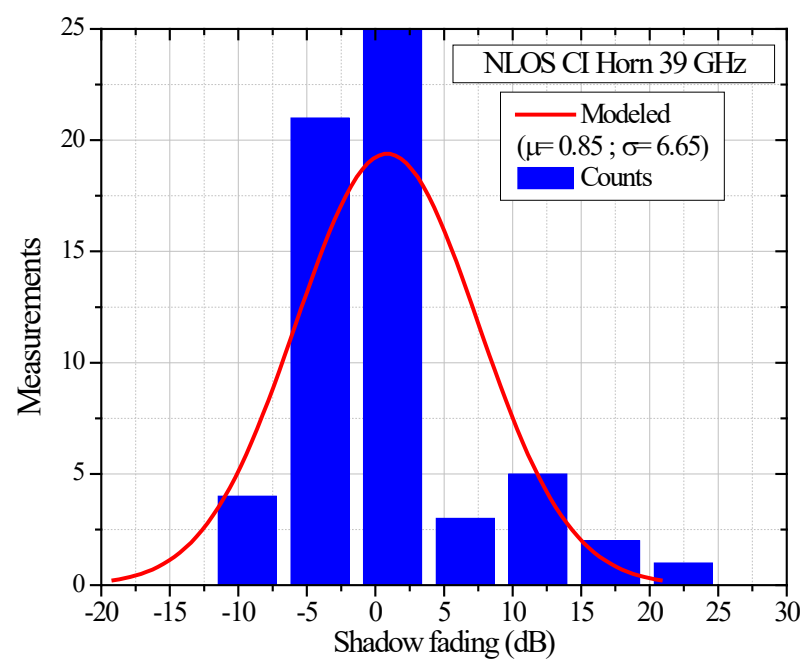

a)

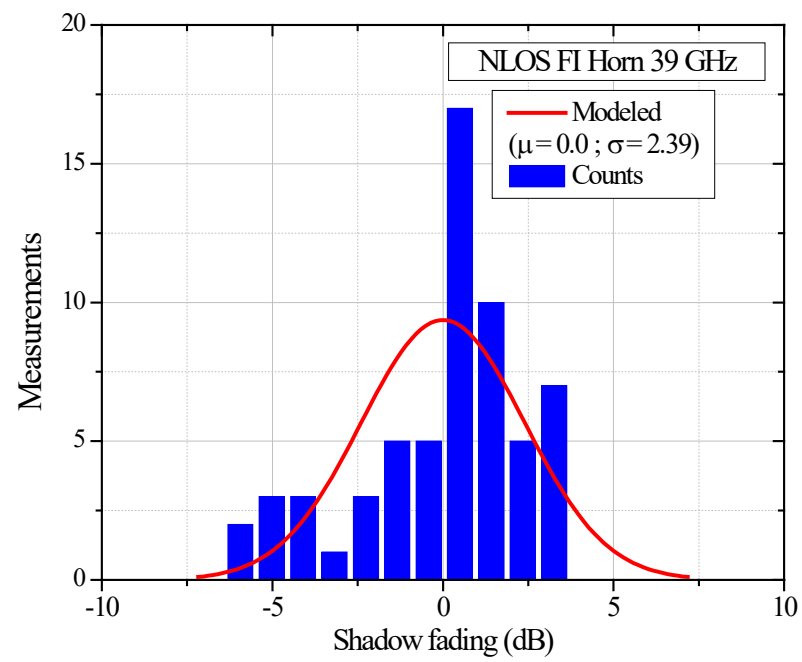

c)

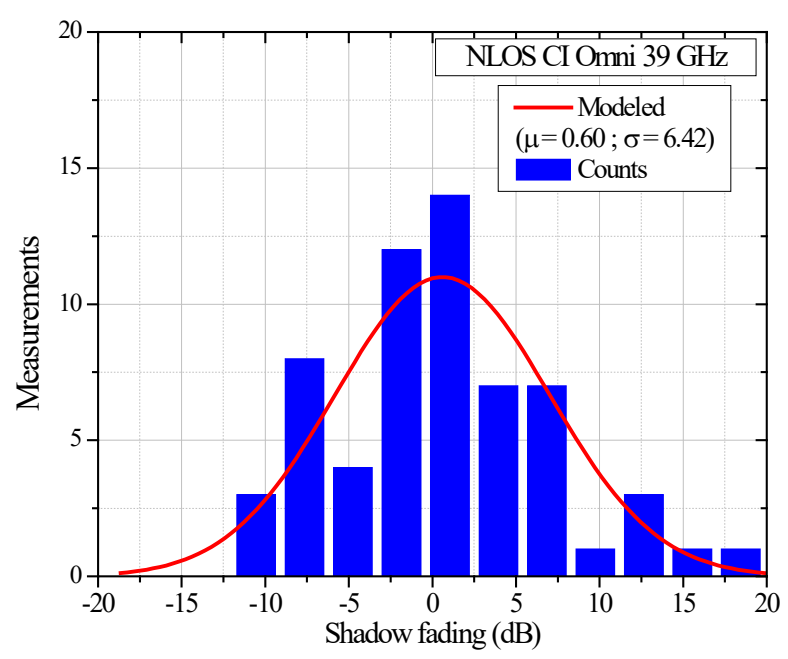

b)

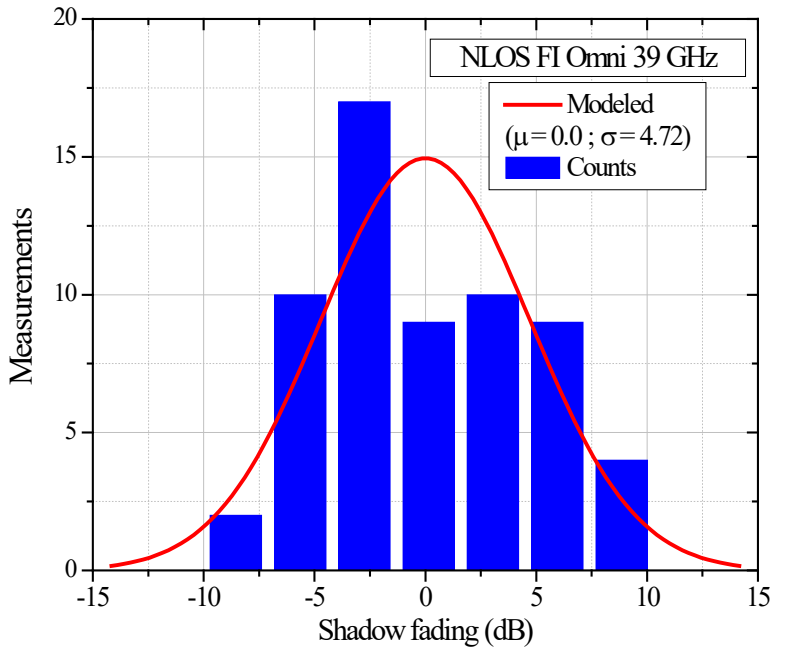

d)

Figure 6.20. $39 \mathrm{GHz}$ NLOS histograms and fitted Gaussian distributions for the fittings and configurations a) CI, Horn, b) CI, Omni, c) FI, Horn and d) FI, Omni. 


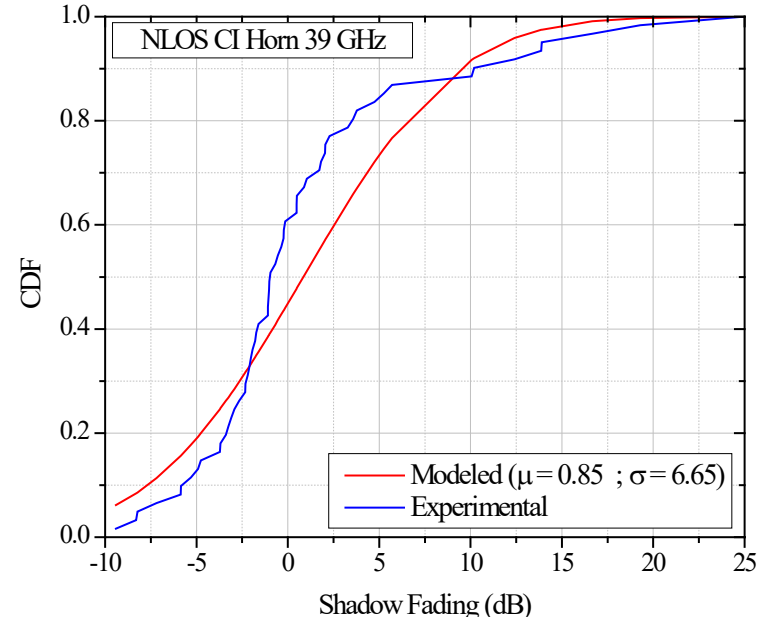

a)

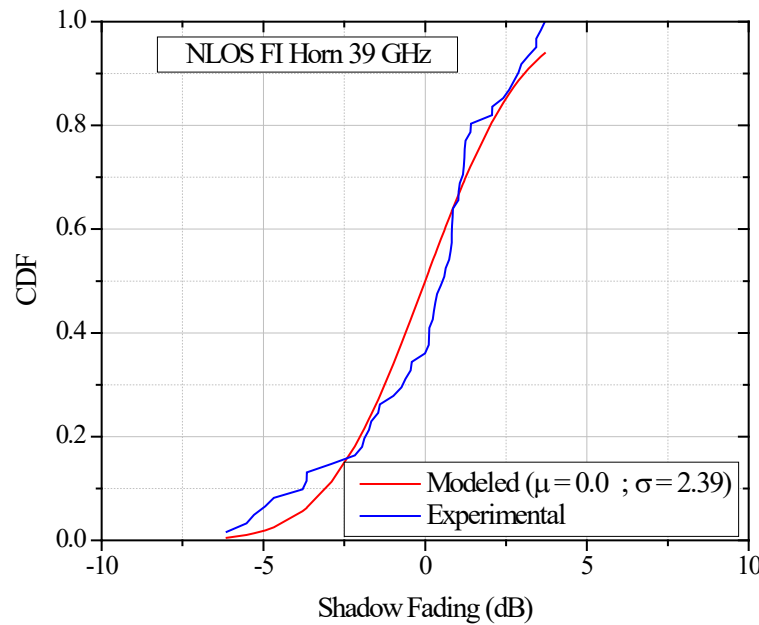

c)

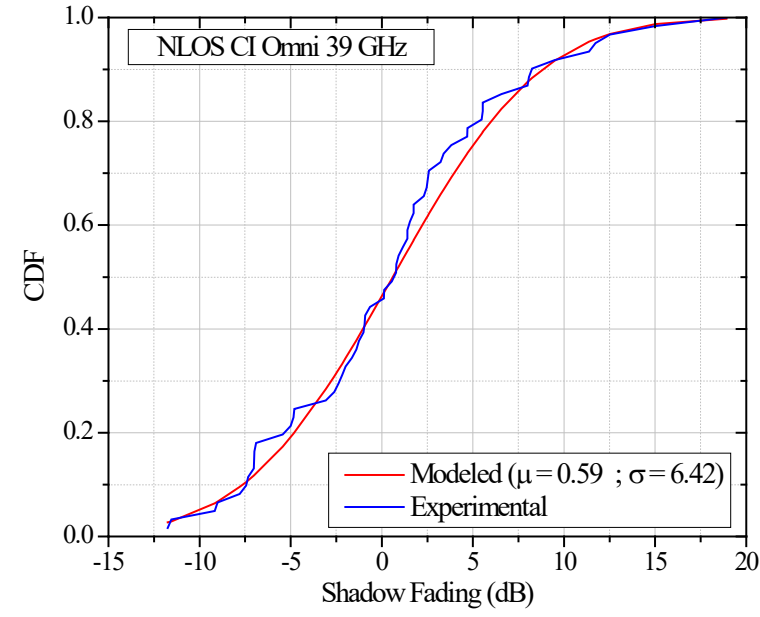

b)

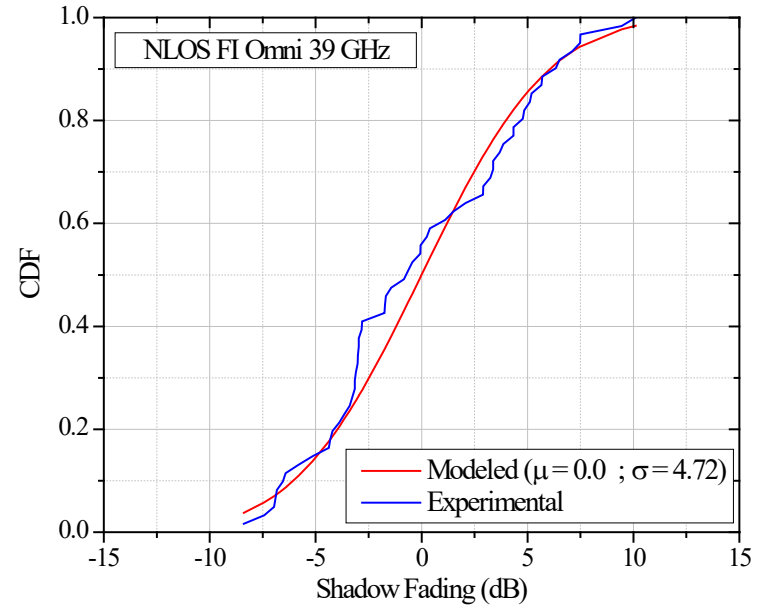

d)

Figure 6.21. $39 \mathrm{GHz}$ NLOS CDF distributions for the fittings and configurations a) CI, Horn, b) CI, Omni, c) FI, Horn and d) FI, Omni.

Table 6.10 collects the mean and standard deviation for the shadow fading obtained from each fitting.

Table 6.10. Statistical values of shadow fading for the InH NLOS measurements

\begin{tabular}{ccccc}
\hline \hline & & Fitting & Mean & Std. \\
\hline $\mathbf{2 6} \mathbf{G H z}$ & Horn & CI & 0.72 & 5.83 \\
& & FI/ABG & 0.0 & 2.58 \\
& \multirow{2}{*}{ Omni } & CI & 0.62 & 6.20 \\
& & FI/ABG & 0.0 & 4.26 \\
\hline $\mathbf{3 9} \mathbf{G H z}$ & \multirow{2}{*}{ Horn } & $\mathbf{C I}$ & 0.85 & 6.65 \\
& & FI/ABG & 0.0 & 2.39 \\
& \multirow{2}{*}{ Omni } & $\mathbf{C I}$ & 0.59 & 6.42 \\
& & FI/ABG & 0.0 & 4.72 \\
\hline \hline
\end{tabular}


Compared with the LOS case, the relatively small amount of measurements limits the histogram binning. Also, it seems that this quantity does not allow a better adjustment of the CI shadow fading to zero-mean Gaussian distributions. However, the $26 \mathrm{GHz}$ CI results are better than the $39 \mathrm{GHz}$ ones, as Table 6.10 shows (i.e. overall, lower means and standard deviations are obtained). From the FI fitting, the zero-mean Gaussian distributions are obtained in all the cases, with lower standard deviations for the Horn configuration; however, the Gaussian distribution does not fit well the experimental histograms and CDFs.

\subsection{MIMO results}

Measurements were gathered in a $2 \times 2$ configuration of omnidirectional antennas inside and in front of the Radiocommunication Laboratory of the ETSI Telecomunicación.

Figure 6.22 shows a zoom of the Lab-room area (limited by a green line) from the floor plan. In this room there are several metal shelves storing different laboratory equipment, computers parts, displays and wires, and wooden tables and metallic/wooden chairs in the three compartments. The two inside divisions (seen as gray lines in the Lab-room area) are made of metal plates that do not reach the ceiling. They divide the place in three compartments.

The receiver unit RX was placed in two alternative positions, which are represented with blue crosses and the transmitter unit TX is represented with a red one. The former is kept fixed at one of the two represented positions, and the latter is moved along the red dashed lines.

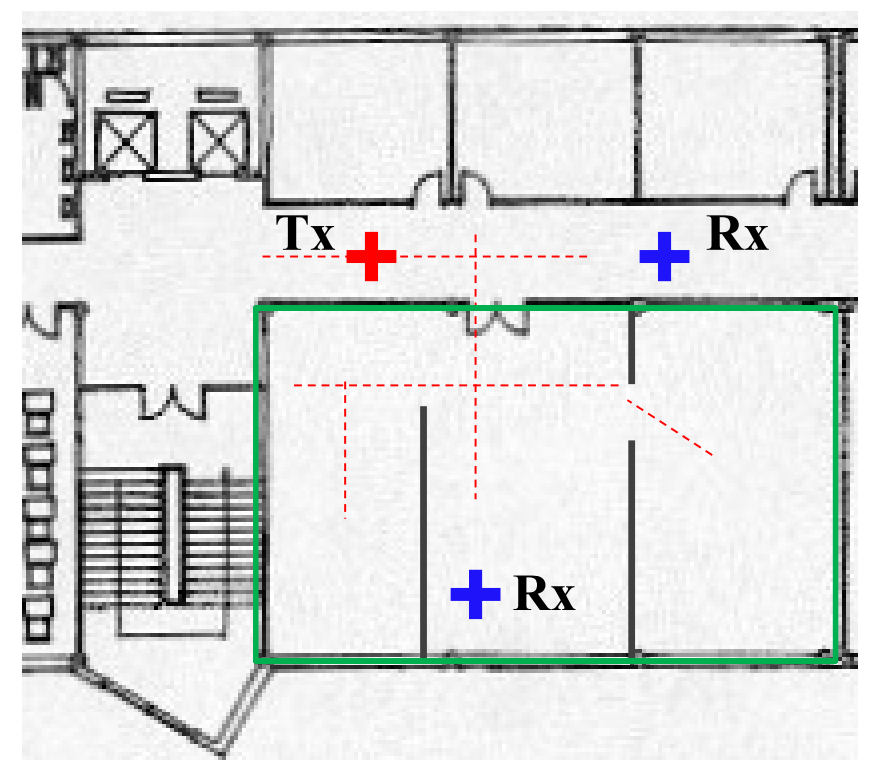

Figure 6.22. MIMO measurements scenario (the receiver RX possible positions are represented with blue crosses, the red cross represents one sample position of the transmitter TX, the red dashed lines represent the movements of TX and the green line delimits the Lab-room area).

The scenario is classified as in the corridor or as in the Lab-room depending on the positions of the receiver. The Lab-room doors were kept open for the in the corridor 
measurements. Later, the measurements gathered for the different TX positions are classified as LOS or NLOS. The amounts of measurements for each condition are given in Table 6.11.

Table 6.11. MIMO measurement classification

\begin{tabular}{ccc}
\hline \hline Scenario & LOS & NLOS \\
\hline In the corridor & 215 & 211 \\
In the Lab-room & 431 & 393 \\
\hline \hline
\end{tabular}

The $\mathbf{H}$ matrix is derived for each measurement of the four possible scenarios of Table 6.11, as was described in sub-section 4.2.5. From $\mathbf{H}$, the obtaining of the diagonal matrix $\boldsymbol{\Lambda}$ (which has the singular values $\lambda_{n}$ in its main diagonal) by means of a SVD is straightforward, as was presented in section 3.4. With the singular values $\lambda_{1}$ and $\lambda_{2}$, the condition number can be computed as:

$$
\text { Condition Number }=\frac{\max _{i} \lambda_{i}}{\min _{i} \lambda_{i}}=\frac{\lambda_{1}}{\lambda_{2}}
$$

The histograms of Figure 6.23 are derived for the condition number obtained from the LOS and NLOS MIMO measurements.

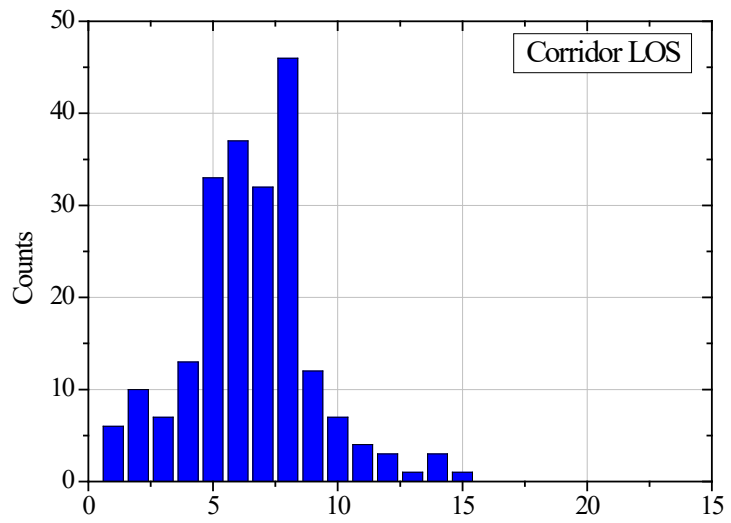

Condition number $(\mathrm{dB})$

a)

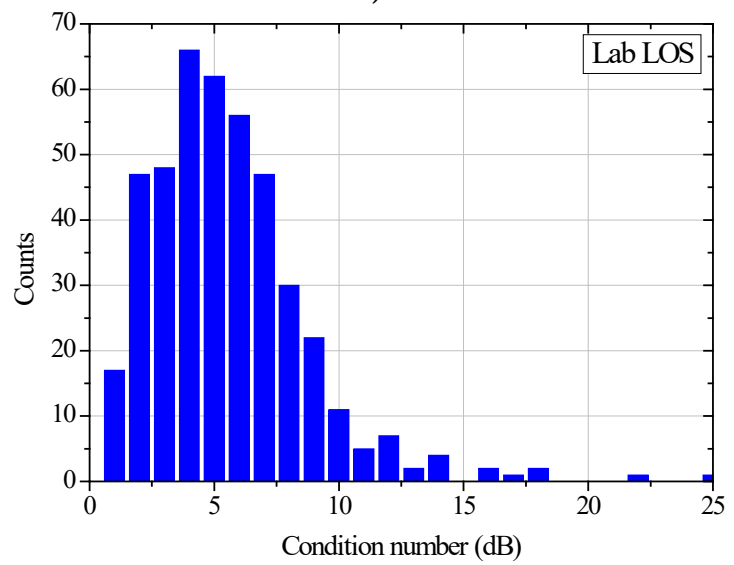

c)

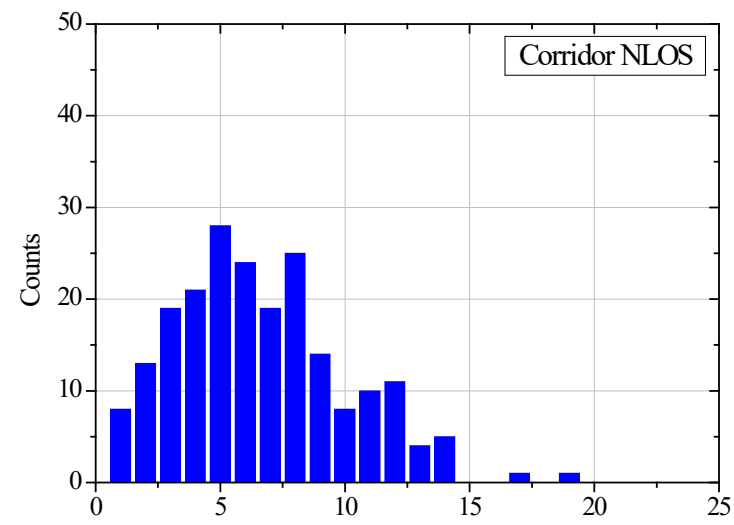

Condition number (dB)

b)

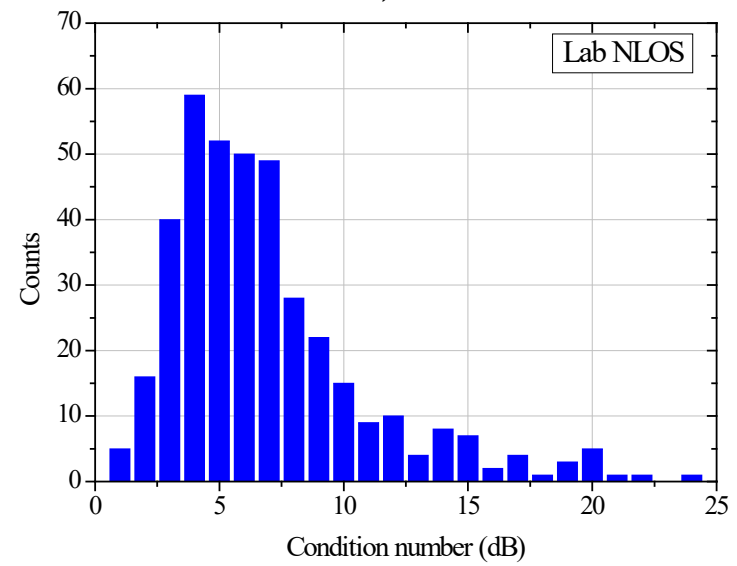

d)

Figure 6.23. Histogram of the conditional number for the different scenarios, a) corridor LOS, b) corridor NLOS, c) Lab LOS and d) Lab NLOS. 
It must be expected that the condition number tends to higher values in the LOS cases, since in such condition $\lambda_{1}$ may be much higher than $\lambda_{2}$ due to the existence of a direct line of visibility between TX and RX. This is partially fulfilled for the corridor measurements, but for the Lab-room scenario the histograms of the condition number for LOS and NLOS are very similar (although a higher amount of high condition numbers is obtained in the Labroom for NLOS). Low condition number values, occasioned by very similar singular values, occur with high frequency. This may allow an increasing in the channel capacity by means of spatial multiplexing.

\subsection{Conclusions}

\section{LOS results}

The amount of measurements for LOS conditions in a corridor at 26 (283 measurements) and $39 \mathrm{GHz}$ (182 measurements) for a 3 to $50 \mathrm{~m}$ distance range seems to be enough to capture the millimeter-wave channel behavior under these conditions.

The PLEs obtained with the Horn configuration (1.86 for $26 \mathrm{GHz}$ and 2.01 for $39 \mathrm{GHz}$ for CI) are very similar to the free space value (i.e. 2). The difference in standard deviations from using a CI or an FI fitting is not so relevant; then, the use of a fitting with more than one coefficient (such as the FI) seems to be un-necessary to model properly the LOS measurements.

Using the CI fitting without a 1-m anchor produces the same results as using an FI (the shadow fading standard deviation for this case is the same as for the FI), since two parameters are employed in both cases. The optimized reference distances are below $1 \mathrm{~m}$. Nevertheless, this value is widely accepted as reference for easy model comparison and a fast computation of the path loss.

The mmMAGIC model predicts a very low shadow fading standard deviation (1.18 dB), which has not been observed in the measurements (the standard deviation of shadow fading for LOS ranges from 3.77 to $5.08 \mathrm{~dB}$, values that can be considered moderate since they are not so different from the ones provided by other models). The model coefficients were modified to an FI-type equation and compared with the obtained ones for an FI fitting, obtaining a good approximation. The ABG-type model coefficients are quite similar to the obtained ones for the single-frequency ABG fitting. Here, the Levenberg-Marquadt algorithm was used for performing the least squares curve fitting with initial values equal to the ABGtype model coefficients.

The FI coefficients derived for the METIS distance range (from 1.5 to $13.4 \mathrm{~m}$ ) are very close to those provided by the METIS stochastic model, especially for $26 \mathrm{GHz}$. Then, the use of this model at these frequencies might be considered (METIS model is only proposed from 50 to $70 \mathrm{GHz})$.

The ITU-R Rec. P.1238-9 LOS model does not provide coefficients for the Corridor scenario at these frequencies. The coefficients obtained from the measurements at $26 \mathrm{GHz}$ can be compared with those provided by the model for an Office scenario ( $n$ of 1.95 and $\sigma_{S F}$ of $2.80 \mathrm{~dB}$ for the model against 1.70 and $4.09 \mathrm{~dB}$ respectively, for the Omni case). The 39 
GHz results may be compared with the $38 \mathrm{GHz}$ coefficients recommended by the model, since no coefficient is given at this frequency. In this case, the Office scenario predictions are close to the results obtained with the Horn configuration ( $n$ of 2.03 and $\sigma_{S F}$ of $4.60 \mathrm{~dB}$ for the model against 2.01 and $5.08 \mathrm{~dB}$ respectively).

The obtaining of PLE values lower than 2 in almost all the cases confirms a waveguidelike propagation effect (produced by multiple reflections from the walls, floor and ceiling) in LOS conditions. This behavior has been seen in several other studies done by other research groups and is taken into account in the 3GPP, ITU-R and 5GCM models. The strength of this effect is variable, but it seems that the type of antenna contributes to that trend: a lower PLE is obtained for omnidirectional antennas.

The use of a dual-slope ABG fitting is only justified for the Horn measurements for both frequencies. However, the improvement obtained with this fitting is too small to compensate the complexity in the modeling (i.e. the shadow fading standard deviation obtained for 26 $\mathrm{GHz}$ is $4.33 \mathrm{~dB}$ whereas for the $\mathrm{CI}$ is $4.62 \mathrm{~dB}$ and for the FI is $4.38 \mathrm{~dB}$ ). A lower standard deviation means a better fitting.

A Gaussian distribution with the same mean and standard deviation has been graphically compared to the respective shadow fading histogram. The shadow fading distribution obtained with the CI fitting deviates more from the zero-mean Gaussian function than the distribution obtained with the FI one. This trend is more pronounced for the Omni cases and at the lowest frequency. However, the Gaussian distribution seems to describe well the shadow fading behavior for both antenna configurations and frequencies.

\section{NLOS results}

Regarding NLOS results, now the difference in the point clouds between configurations is higher than in the LOS case; this may be conditioned to a higher amount of energy lost in the Horn case. All the obtained CI PLEs (ranging from 3.16 to 4.11) are higher than 2 (the one for FS), a behavior that is expected.

The differences between the CI and FI fittings are higher than in the LOS case. They are notable at short and middle distances (from 3 to $30 \mathrm{~m}$ ). The use of an FSPL anchor in the CI has to do with that, since it is clear that the propagation in NLOS largely deviates from FS conditions for the previously mentioned distances. Here, the more complex formulations of $\mathrm{CIF}$ and ABG fittings for single frequency give the same curves and $\sigma_{S F}$ 's as the CI and FI fittings, respectively.

The 3GPP model is the only one that gives a CI-type path loss equation, with a PLE of 3.19 and a shadow fading standard deviation much higher than in LOS cases (8.29 dB). This high $\sigma_{S F}$ is seen in all CI fittings, but with values lower than the provided by the model. Although the ITU-R model is CI-based, it does not provide PLEs for NLOS conditions at the frequencies and scenario in question, only a basic comparison can be done with coefficients given for near frequencies and different scenarios (Office and Commercial). It seems that the difference in scenario produces a noticeable difference in the coefficients (i.e. at $38 \mathrm{GHz}$ the PLE is 2.96 for Office and 2.59 for Commercial, whereas the obtained for $39 \mathrm{GHz}$ are 4.11 and 3.39). 
The comparison with the METIS model (FI-based) without taking into account the limits in distance and frequency gives moderate approximations for the floating-intercept coefficient and the shadow fading standard deviation, but deviates more for the distance-dependent coefficient.

The ABG coefficients for the 5GCM, 3GPP and mmMAGIC models are comparable to the derived ones for the single frequencies. Also, the $\sigma_{S F}$ is much higher for the models than for the ABG fittings. The derived coefficients were obtained using, as in the LOS case, the Levenberg-Marquadt algorithm and initial values close to the coefficients provided by the models. However, if the algorithm or even the initial values are changed, only the derived $\alpha$ and $\gamma$ coefficients change whereas $\beta$ (the PLE) remains unchanged (as occurs for LOS). This occurs since a multi-frequency ABG fitting is not performed (the case in which the two first terms could be determined univocally).

Again, the dual-slope ABG fitting can be applied to the Horn configuration at both frequencies; but the obtained improvement, compared with the single-slope FI/ABG fittings, is small. When comparing these results with the 5GCM dual-slope indoor models some remarkable differences arise regarding the break point distance. For these models, very different break distances of 6.9 and $147.0 \mathrm{~m}$ were set up for Office and Shopping-Mall scenarios (the framing of the used Corridor scenario in these classifications may be questioned, but the model does not provide an equation for this environment), and the obtained ones are not in such extreme values, rather in an intermediate distance value (around $30 \mathrm{~m}$ ). The difference in the scenario may impact also in the difference of the shadow fading standard deviations, which can be expected to be higher in offices and shopping-malls (as the models predict) than in corridors. Also, the PLEs are considerably small (1.08 for $26 \mathrm{GHz}$ and 0.44 for $39 \mathrm{GHz}$ ) compared with the model ones (1.70 for Office and 2.90 for Shopping-Mall).

The obtained shadow fading standard deviation for the FI fitting is higher for the Omni case than for the Horn at both frequencies. This may be related to the antenna radiation patterns. Omnidirectional antennas are designed to receive signal components coming from a wide angular range, produced by different propagation mechanisms such as reflections, diffractions and/or diffuse scattering. These components can be summed up constructively or destructively. On the other hand horn antennas have a narrow angular range, then the probability of receiving components more focused, which are traveling together instead of interfering one to each other, is higher. The possible interfering multi-paths in this case are just out of the receiving angular sector of the horn antenna.

The Gaussian distributions derived with the shadow fading means and standard deviations do not represent well the experimental histograms and CDFs. In the CI case, the zero-mean Gaussian is better approximated for $26 \mathrm{GHz}$ than for $39 \mathrm{GHz}$ in general. Nevertheless, the approximation in all cases is worse than in LOS. This may be due to the relatively small amount of measurements, and also to other causes.

\section{MIMO results}

The MIMO channel capacity was tested using the condition number. The available equipment limits a more thorough analysis on this topic. The measurements taken with the 
receiver in the corridor at $39 \mathrm{GHz}$ give results close to the expectation from the theory: the histogram of the condition number is more populated at higher values for LOS, produced by the existence of a line of sight between TX and RX which conditions that $\lambda_{1}$ is in theory much higher than $\lambda_{2}$ in most of the cases. This trend is not seen in the Laboratory-room histogram; then, it seems that the existence of a line of sight in this condition is not predominant and some reflections may compensate its lack for NLOS conditions.

The obtaining of a more equally distributed histogram (the one that can be considered as such is the NLOS in the Corridor) means that there are two paths with similar SNR; then the capacity of the channel may be enhanced. 


\section{Conclusions and future lines of work}

\section{Introduction}

After presenting the main results of the Thesis related to satellite and indoor propagation in Chapters 5 and 6, some general conclusions and the main open issues for further researches are given in this chapter. Also, the publications (in journals or conferences), the contributions to ITU-R SG3 Working Parties and the participation in research projects carried out along this research are presented.

\subsection{Conclusions}

\section{Satellite propagation}

Regarding satellite propagation, the core of the analyses are the attenuation results obtained from the Ka-band KA-SAT and the Q-band Alphasat experiment. The measurement database (five years of KA-SAT and four years of Alphasat) has a very high availability (higher than the $97.6 \%$ of the period for both cases), which increases its statistical importance since studies with such amount of years are not frequent. Both excess and total attenuation (only for the Q-band case) time series have been derived. This PhD Thesis is based on analyses carried out with the excess attenuation measurements.

Several ancillary instruments (i.e., pluviometer, disdrometer and rain radar) are also used to obtain the necessary information regarding rain, which produces the main attenuation 
contribution at these frequencies. If there were no rain information, the rainfall rate distributions could be derived with some prediction methods, three of which have been tested here: the Rec. ITU-R P.837-6, the Rec. ITU-R P.837-7 and MORSE models. It can be said that the recent Rec. ITU-R P.837-7 and the MORSE models do not give the best overall approximation to the experimental rainfall rate distributions, which is obtained with the Rec. ITU-R P.837-6 model. However, these models improve the prediction accuracy for the low probabilities, which are related with the occurrence of convective rain events.

Some of the main original contributions of this Thesis are related to fade and inter-fade duration modeling. Since the tested fade duration models (the ITU-R and CRC) do not give accurately enough predictions, a modeling effort was done with the objective of proposing and evaluating possible model improvements. With this aim, several types of fittings were tested by finding the best coefficients for several function combinations. From it, the two lognormal distribution combination gives the best approximation for the FD probability of occurrence either for the Ka- or the Q-band results. Although the CRC model uses such combination, its parameters could be improved: currently its predictions are not accurate for the Madrid case, especially for Q-band.

Another contribution is the procedure developed for estimating the fading time distribution from the two log-normal function parameters obtained from the probability of occurrence modeling. This gives results comparable to those of the fading time fitting with the advantage that it relies on the physical basis that the total fading time depends on the distribution of the number of fade events.

Regarding inter-fade duration, the author's major contribution is the finding that a two log-normal distribution combination is suitable for fitting the inter-fade probability of occurrence and inter-fading time distributions for durations shorter than $10^{6} \mathrm{~s}$. Taking this into account, it seems possible the development of an inter-fade duration model with the previously mentioned combination, valid for durations up to that limit. If longer durations are required, a three log-normal distribution combination seems to be a good option. Durations longer than $10^{6} \mathrm{~s}$ may have a low practical importance and the addition of another term to the model may not compensate the increase in the complexity.

The performance of time and orbital diversity has been analyzed using the measurements registered in these experiments. The possible time diversity gain is higher for the Q-band, in which a higher attenuation occurs, and for time percentages lower than $0.01 \%$ (it can reach up to $17 \mathrm{~dB}$ for a delay of $600 \mathrm{~s}$ and $0.01 \%$ percentage of time). For probabilities higher than $0.1 \%$, the gain is considerably small for both bands, barely reaching $1 \mathrm{~dB}$ for Ka-band and $4 \mathrm{~dB}$ for Q-band with the same $600 \mathrm{~s}$ delay. The rain conditions in Madrid, with a relatively low probability of rain $(2.22 \%$ for the 4 year period) and a relevant presence of stratiform events with long-durations, may produce this trend, since they can only be mitigated with very long delays.

The four analyzed time diversity models (Matricciani, ONERA, Greece and Joint Probability) give similarly good results when compared to the experimental distributions. Overall, the predictions are better for the Q-band than for the Ka-band, even though they were developed using data mainly gathered at lower frequencies. Also, most of the models 
give better approximations for smaller time delays and work worse for longer delays. The Matricciani TD model gives the best approximation in the Ka-band and the Greece and ONERA models give better ones for the Q-band.

The orbital diversity results were derived after a frequency scaling procedure developed in the course of this research by making use of the availability of concurrent measurements at the two frequency bands. The two orbital diversity models that were tested (Matricciani OD and NTUA) show similarly good results, despite the fact that they were developed and tested using data from experiments at lower frequencies.

The main original contribution regarding the aforementioned diversity techniques is the comparison between the models by itself, especially for the Q-band where results are scarce. The amount of available data allows such kind of analyzes.

From the comparison of time and orbital diversity, it seems that TD gain with a delay ranging from 3 to 5 minutes may emulate the OD gain. This allows the use of both techniques in a complementary manner, depending on the delay that the application can tolerate. Also, it may be anticipated that for a larger aperture angle between the links, the gain obtained with OD should be similar to the obtained by applying TD with a longer time delay.

A high monthly and yearly variability was observed for the analyzed periods, especially for first-order statistics (rainfall rate and rain attenuation). This Thesis has been focused on the fade dynamics variability. Here, a parameter $V$ has been used for assessing the FD and IFD variability at several attenuation thresholds. For both statistics, the parameter has a similar order of magnitude. Also, the normalized distributions (probability of occurrence and fraction of fading/inter-fading time) have less variability than the absolute distributions (number of fade/inter-fade events and total time).

\section{Millimeter-wave Indoor channel results}

LOS and NLOS measurements taken in a corridor scenario at 26 and $39 \mathrm{GHz}$ have been presented. The major work done by the author is focused in the design, setting-up, calibration, gathering of measurements and the data analysis.

The basis of the measurement process is the calibration, which was done using horn antennas at both ends and referencing the measurements to the free space condition. The measurements have been collected for two configurations: one with horn antennas at both ends (Horn) and the other with one horn antenna at the transmitter and one omnidirectional antenna at the receiver side (Omni).

A path loss analysis has been done by using several fittings (such as the CI, FI and ABG) to derive the coefficients that best describe the different conditions.

For LOS condition, a very similar error is found with the CI and FI fittings; then, using a second coefficient (as the FI does) or more to describe the measurements may not be necessary for the experiment conditions. Also, the waveguide-like propagation effect (produced by multiple reflections from walls, floor and ceiling) is seen in general, meaning that the propagation is better than in free space. 
The obtained LOS fitting coefficients were compared with those provided by the available millimeter-wave path loss models, giving good approximations. The major similarities were obtained between the 3GPP and 5GCM Office models and the CI Omni case at $26 \mathrm{GHz}$. Maybe the use of an omnidirectional antenna conditions this result. Also, the METIS model was extended to both frequencies (but keeping its recommended distance range) with very good results. Moreover, the ABG single-frequency fitting produces coefficients similar to those provided by the mmMAGIC model, which also gives a very low standard deviation not observed in the measurements.

From the NLOS measurements, the difference in the point clouds of the Omni and Horn configurations is relatively higher than in the LOS case, may be conditioned by a higher amount of energy lost in the latter due to the directivity of the antenna.

The expected increase in the PLE for CI fittings is seen in all cases. The difference between the CI and FI NLOS fittings is higher than in LOS, especially at short and middle distances (up to approximately $30 \mathrm{~m}$ ) and at $39 \mathrm{GHz}$. The use of a FSPL anchor in the CI is the main reason of that, since it is clear that the propagation in NLOS largely deviates from FS conditions, particularly for the previously mentioned distances.

The ABG-based models (i.e. 5GCM, 3GPP and mmMAGIC) give in all cases PLEs higher than the obtained from the measurements at each frequency. Maybe this is conditioned by the scenario (the models do not provide coefficient for Corridor). Also, all models provide higher shadow fading standard deviations than the obtained ones.

In the case of the ITU-R Rec. P.1238-9 model, no coefficients are provided for Corridor scenario at these frequencies. Then, in the author's opinion, the coefficients obtained for LOS and NLOS conditions may serve to fill that gap.

The dual-slope ABG fitting only returns consistent results for the Horn configuration at both frequencies for LOS and NLOS. Nevertheless, such complex formulation of path loss does not enhance significantly the modeling (the obtained reduction of the shadow fading is small in all cases, around $0.1 \mathrm{~dB})$.

For the LOS shadow fading analysis, the measured shadow fading is well described with a Gaussian distribution in all cases. Also, the shadow fading standard deviation is higher for the Horn than for the Omni case at each frequency.

Regarding NLOS shadow fading, the obtained one from the FI has a standard deviation higher for the Omni than for the Horn case. This may be produced by the antenna radiation patterns as it was previously explained. The zero-mean Gaussian distribution is only obtained with the fittings that use more than one coefficient (such as the FI/ABG). In the NLOS case, the graphical representation of the Gaussian distribution does not describe well the respective histogram. Maybe the limited amount of measurement conditions this behavior.

The MIMO channel capacity has been tested using the condition number to the available data collected at $39 \mathrm{GHz}$. The measurements taken with the receiver in the corridor gave a result close to the theoretical expectation: the condition number histogram is more populated at lower values in NLOS and at higher ones in LOS, produced by the existence of a line of sight between TX and RX. Also, the histogram of NLOS is more distributed in a wider range 
of bins. These trends are not seen in the Laboratory-room histograms, then, it seems that the existence of a line of sight in this condition is not predominant and some reflections may compensate the lack of this for NLOS conditions.

\section{2. $\quad$ Future works and open issues}

\section{Satellite propagation}

It is expected that the KA-SAT and Alphasat measurement campaigns will continue for some more years. With these, the presented results can be refined. Nevertheless, it is presumed that the main conclusions given here will hold. Other propagation effects, such as cloud attenuation and first-order statistics of rain attenuation, can be studied taking advantage of the availability of the larger number of experimental measurements.

After the fade and inter-fade duration modeling efforts carried out in this Thesis, it seems appropriate to expand them using data from other sites, elevation angles and frequencies. The correlation of the results with some meteorological parameters may lead to a model improvement for the fade duration case and to a model development for inter-fade duration.

The presented procedure for deriving the distribution of the total fraction of fading time from the two log-normal distribution function coefficients obtained for the probability of occurrence might be extended for modeling the fraction of inter-fading time. Some modifications must be applied to that end since the conditions are different, i.e. a two lognormal distribution function serves well to model inter-fade events up to $10^{6} \mathrm{~s}$ but is not good enough for longer events, less frequent but representing a high percentage of the total interfading time.

The time and orbital diversity modeling may give more accurate results at the time that more years of measurements were available. It is interesting to continue testing the models to be able to propose possible improvements.

Also, the variability analysis of several statistics (especially fade dynamics) may be refined if more years of measurements were available.

\section{Millimeter-wave Indoor channel results}

Although the amounts of collected path loss measurements are relatively high, a higher number could improve some conclusions, e.g. regarding a more accurate shadow fading standard deviation or the possible existence of a break point distance in the NLOS measurements. Also, the application of full multi-frequency fittings (such as the ABG and CIF) to the path loss measurements could be done if measurements at a higher number of frequencies are taken.

Due to the importance of the millimeter-wave bands in the near future, it is also necessary to expand the measurements to other frequencies of interest (such as the identified ones by the WRC-15 or the future WRC-19) and scenarios, such as outdoor and outdoor-toindoor (with the correspondent penetration loss study).

The study of the diffraction produced by objects and the penetration loss in the indoor scenario are research issues to have into consideration in the future. 
Regarding MIMO, a much higher amount of measurements may serve to confirm the obtained trends and carry out more elaborated analyses. Also, the channel capacity may be evaluated.

\subsection{Publications and scientific work}

Along the period of development of this Thesis, a number of papers and/or communications presenting partial results have been published. They are collected here together with a brief description of each one:

\section{Journal articles}

- J.M. Garcia-Rubia, J.M. Riera, P. Garcia-del-Pino, D. Pimienta-del-Valle, and G.A. Siles, "Fade and Inter-Fade Duration Characteristics in a Slant-Path Ka-Band Link," IEEE Transactions on Antennas and Propagation, vol. 65, no. 12, pp. 7198-7206, December 2017.

Seven-year results regarding fade and inter-fade duration with EUTELSAT Hot Bird $13 \mathrm{~A}$ data were presented. The author's main contribution to this article was the modeling of fade duration probability of occurrence and fraction of time of this experiment with the function combinations of sub-section 2.5.1. Some ideas treated here, such as the use of the $V$ parameter of sub-section 2.7 for assessing the fade duration variability, have been extended to this Thesis to both fade and inter-fade duration variability analysis for KA-SAT and Alphasat results.

- D. Pimienta-del-Valle, J.M. Riera, P. Garcia-del-Pino, and G.A. Siles, "Three-year fade and inter-fade duration statistics from the Q-band Alphasat propagation experiment in Madrid," International Journal of Satellite Communication and Networking, 2018; 1-17.

In this article, a thorough presentation about the Alphasat experiment in Madrid (as shown in sub-section 4.1.3) was done. Also, the first- and second-order statistical results were given for a three-year period using excess attenuation data. Fade duration results were compared with the ITU-R and CRC models and fitted with the function combinations of sub-section 2.5.1. Furthermore, the procedure of Annex 2 of this Thesis for deriving the fading time distribution was presented.

- D. Pimienta-del-Valle, J.M. Riera, and P. Garcia-del-Pino, "Ka- and Q/V-Band Inter-Fade Duration Modeling," IEEE Transactions on Antennas and Propagation, vol. 66, no. 12, pp. 7511-7514, December 2018.

Here, the modeling of the inter-fade duration probability of occurrence distribution was accomplished. Beacon data from previous experiments (one year of measurements of Italsat and seven years of measurements of EUTELSAT Hot Bird 13A) and KASAT (four years) and Alphasat (four years) experiments were used. For all experiments, it was proved that the experimental probability of occurrence distribution can be modeled with a two log-normal function up to durations of $10^{6} \mathrm{~s}$ 
and that for modeling longer durations a three log-normal function is a very good choice.

- D. Pimienta-del-Valle, J.M. Riera, and P. Garcia-del-Pino, "Time and Orbital Diversity assessment with Ka- and Q-Band slant-path propagation experiments in Madrid," IEEE Transactions on Antennas and Propagation, vol. 67, no.2, pp.11931201, February 2019.

This paper presents time and orbital diversity results for a three-year period, the first for the Ka- and Q-band and the second for the Q-band. Excess attenuation data for the KA-SAT and Alphasat beacons were used. The models of sub-sections 2.6.1 and 2.6.2 were applied for this article. Also, the procedure of scaling in frequency the Kaband results to the Q-band was carried out.

\section{Publications in international conferences}

- P. Garcia-del-Pino, D. Pimienta-del-Valle, A. Benarroch, and J.M. Riera, "Evaluation of recent prediction models using a long-term database of rainfall rate data," in 2017 11th European Conference on Antennas and Propagation (EUCAP), Paris, France 2017, pp. 20-24.

The performance of a rainfall rate model (presented in sub-section 2.3.3.3) and the inter-annual variability prediction method recommended by the ITU-R (addressed in sub-section 2.7) was carried out using rain gauge data of 16 years. The ITU-R variability model was also employed to assess monthly and seasonal variability.

- J. M. Riera; D. Pimienta-del-Valle; P. Garcia-del-Pino; G. A. Siles; A. Benarroch. "Alphasat propagation experiment in Madrid: Results on excess and total attenuation," in 2017 11th European Conference on Antennas and Propagation (EUCAP), Paris, France 2017, pp. 1-4.

Monthly and yearly results on excess and total attenuation for two years of measurement with the Q-band Alphasat beacon receiver were presented. From these, a high variability between the monthly attenuation distributions in a year and from one year to another was shown.

- D. Pimienta-del-Valle; A. Benarroch, J. M. Riera, P. Garcia-del-Pino. "First results of total attenuation distributions and fade dynamics from the Alphasat propagation experiment in Madrid," in XXXII URSI General Assembly $\&$ Scientific Symposium, Montreal, Canada 2017.

The first preliminary results on total attenuation and fade duration for two years of total attenuation data from the Q-band Alphasat experiment in Madrid were presented. These were also compared with the ITU-R and CRC fade duration models.

- D. Pimienta-del-Valle, J. M. Riera, P. Garcia-del-Pino, G. A. Siles. "Alphasat Experiment in Madrid: Modeling Considerations on Fade and Inter-Fade Durations," in 2018 12th European Conference on Antennas and Propagation (EUCAP), London, United Kingdom 2018, pp. 1-5. 
Preliminary results for fade and inter-fade duration modeling using excess attenuation data for three years of the Q-band Alphasat experiment in Madrid were presented. Also, the CRC and ITU-R fade duration models were tested.

- D. Pimienta-del-Valle, J. M. Riera, P. Garcia-del-Pino, G. A. Siles. "A Four-Year Variability Study for Ka- And Q-band Slant Path Propagation Experiments in Madrid," (Accepted) in 13th European Conference on Antennas and Propagation (EUCAP), Krakow, Poland 2019.

This communication assesses the variability of the number of fade and inter-fade events for a 4-year concurrent period between the Ka-band KA-SAT experiment and the Q-band Alphasat one. The $V$ parameter of sub-section 2.7 was used to fulfill that objective. Furthermore, rainfall rate and excess attenuation variability were evaluated.

- D. Pimienta-del-Valle, S. Hernández-Sáenz, P. J. Saiz Coronado, L. Mendo Tomas, P. Garcia-del-Pino, J. M. Riera. "Indoor Path Loss Measurements at the 5G MillimeterWave Bands of 26 and $39 \mathrm{GHz}, "$ (Accepted) in 13th European Conference on Antennas and Propagation (EUCAP), Krakow, Poland 2019.

The path loss measurement results in LOS condition for 26 and $39 \mathrm{GHz}$ are presented here. Measurements were fitted with the CI and FI models (addressed in sub-sections 3.2.1 and 3.2.2 respectively), and the obtained coefficients compared with the 3GPP TR 38.901, 5GCM and mmMAGIC models. Also, the main equipment characteristics and the calibration procedure (treated in sub-section 4.2) are given.

- J. Villegas Gutierrez, D. Pimienta-del-Valle, C. Oestges. "Physical Identification of Common Clusters for Outdoor Microcells," (Accepted) in 13th European Conference on Antennas and Propagation (EUCAP), Krakow, Poland 2019.

This work is a result of the colaboration with UCLouvain researchers, frammed in the doctoral stay conducted during the research period. A new approach for identifying physically interacting objects and defining common clusters are proposed.

Publications in national conferences

- D. Pimienta-del-Valle, J. M. Riera, P. Garcia-del-Pino, A. Benarroch. "Resultados del experimento de propagación con Alphasat en Madrid: atenuación total y dinámica de desvanecimientos," in XXXII Simposium Nacional de la Unión Científica Internacional de Radio (URSI), Cartagena, Spain 2017, pp. 1-4.

Total attenuation results for a two-year period with the Q-band Alphasat experiment were presented. Also, the fade duration results were compared with the ITU-R and CRC models and fade slope results with the ITU-R model (presented in sub-section 2.5.3). 
- D. Pimienta-del-Valle, J. M. Riera, P. Garcia-del-Pino, G. A. Siles. "Experimento Alphasat en Madrid: Resultados y modelado de la probabilidad de ocurrencia de los desvanecimientos," in XXXIII Simposium Nacional de la Unión Científica Internacional de Radio (URSI) Granada, Spain 2018, pp. 1-4.

The latest results at that time regarding Alphasat and the probability of occurrence modeling (either for fade or inter-fade durations) were presented. Excess attenuation data for a three-year period were used.

- P. J. Saiz Coronado, D. Pimienta-del-Valle, L. Mendo Tomas, P. Garcia-del-Pino, J. M. Riera. "Medidas de atenuación en entorno de interiores para ondas milimétricas: montaje experimental y primeros resultados," in XXXIII Simposium Nacional de la Unión Científica Internacional de Radio (URSI) Granada, Spain 2018, pp. 1-4.

The first results regarding LOS measurements in the corridor scenario at $39 \mathrm{GHz}$ were presented. The same Horn and Omni configurations of Chapter 6 were used. The experimental setup, data processing and calibration procedure were addressed thoroughly.

Contributions to ITU-R SG3 Working Parties

- Spain ITU-R contribution. "Updated rainfall rate statistics for DBSG3 Table IV-1". Document 3M/100E. Study Groups 3 - Radiowave propagation, June 2016.

- Spain ITU-R contribution to the Propagation Data Banks. "Rain rate and slant-path rain attenuation measurements in Madrid". Document 3J/98-E 3M/166-E, Study Groups 3 - Radiowave propagation, March 2017.

- Spain ITU-R contribution. "Evaluation of recent rainfall rate and variability prediction models using a long-term database of rainfall rate". Document 3J/99-E 3M/167-E, Study Groups 3 - Radiowave propagation, March 2017.

Participation in research projects

- MINECO project. "Experimental characterization of atmospheric propagation and channel modeling in millimeter-wave bands, oriented to their future use in fixed and mobile 5G communication networks," Project Reference TEC2014-57821-R, Spain

- ESA project. "Development of high order propagation models for multimedia satellite communication systems," Contract ESTEC 4000107135/12/NL/LVH, 2016-17.

- Comunidad de Madrid project. "Space debris radar- radar para detección de basura espacial," Project Reference S2013/ICE-3000, Spain. 



\section{Annex 1 ONERA Time diversity model}

With the parameters given in the sub-section 2.6.1.4, the ONERA time diversity model is implemented as follows [229]:

Step A1.1: First, the rain probability distributions conditioned to the probability of rain in the average year is calculated:

$$
P=\frac{P_{0}}{100} Q\left(\frac{\ln \left(A_{t h}\right)-m}{\sigma}\right)
$$

Step A1.2: The following expression is applied:

$$
c_{G}=e^{-\beta \delta t}
$$

if:

$$
c_{G} \leq 0.0001 \text { then } P_{\delta t}=100 P^{2}(\%)
$$

Otherwise, Step A1.3 to Step A1.6 are performed.

Step A1.3: Calculate:

$$
\alpha_{t h}=Q^{-1}(P)
$$

Step A1.4: Calculate:

$$
U=\frac{1-c_{G}^{2}}{c_{G}^{2}} \quad V=\frac{\alpha_{t h}}{c_{G}} \sqrt{2 U} \quad W=-\frac{\alpha_{t h}^{2}}{2 c_{G}^{2}} \quad K=\frac{1}{2} \sqrt{\frac{U}{\pi}}
$$


Step A1.5: Calculate:

$$
a=\frac{653}{720} \quad \omega_{1}=\frac{928}{1175} \quad b_{1}=\frac{4532}{6043} \quad \omega_{2}=\left(1-\omega_{1}\right) \quad b_{2}=\frac{\frac{2}{\sqrt{\pi}}-b_{1} \omega_{1}}{\omega_{2}}
$$

Step A1.6: Calculate:

$$
X_{M}=\frac{\alpha_{t h}}{\sqrt{2}} \sqrt{\frac{1-c_{G}}{1+c_{G}}}
$$

If $\mathrm{X}_{\mathrm{M}} \leq 0$, perform Step A1.6-1

If $\mathrm{X}_{\mathrm{M}}>0$, perform Step A1.6-2

Step A1.6-1: Calculate:

$$
\begin{aligned}
& A=K\left[2 \sqrt{\frac{\pi}{U}} e^{V^{2}+W} Q\left(\frac{V-2 U X_{M}}{\sqrt{2 U}}\right)-\omega_{1} \sqrt{\frac{\pi}{U_{1}}} e^{\frac{V_{1}{ }^{2}}{4 U_{1}}+W} Q\left(\frac{V_{1}-2 U_{1} X_{M}}{\sqrt{2 U_{1}}}\right)\right. \\
& \left.-\omega_{2} \sqrt{\frac{\pi}{U_{1}}} e^{\frac{V_{2}^{2}}{4 U_{1}}+W} Q\left(\frac{V_{2}-2 U_{1} X_{M}}{\sqrt{2 U_{1}}}\right)\right]
\end{aligned}
$$

with:

$$
U_{1}=U+a \quad V_{1}=V+b_{1} \quad V_{2}=V+b_{2}
$$

Then:

$$
P_{\delta t}=100 \cdot A(\%)
$$

Step A1.6-2: Calculate:

$$
\begin{gathered}
A=K\left[2 \sqrt{\frac{\pi}{U}} e^{\frac{V^{2}}{4 U^{+}} W} Q\left(\frac{V}{\sqrt{2 U}}\right)-\omega_{1} \sqrt{\frac{\pi}{U_{1}}} e^{\frac{V_{1}{ }^{2}}{4 U_{1}}+W} Q\left(\frac{V_{1}}{\sqrt{2 U_{1}}}\right)\right. \\
\left.-\omega_{2} \sqrt{\frac{\pi}{U_{1}}} e^{\frac{V_{2}^{2}}{4 U_{1}}+W} Q\left(\frac{V_{2}}{\sqrt{2 U_{1}}}\right)\right]
\end{gathered}
$$

and:

$$
\begin{aligned}
B=K\left[\omega_{1} \sqrt{\frac{\pi}{U_{1}}} e^{\frac{V_{1}^{2}}{4 U_{1}}+W}\left(Q\left(\frac{V_{1}-2 U_{1} X_{M}}{\sqrt{2 U_{1}}}\right)-Q\left(\frac{V_{1}}{\sqrt{2 U_{1}}}\right)\right)\right. \\
\left.+\omega_{2} \sqrt{\frac{\pi}{U_{1}}} e^{\frac{V_{2}^{2}}{4 U_{1}}+W}\left(Q\left(\frac{V_{2}-2 U_{1} X_{M}}{\sqrt{2 U_{1}}}\right)-Q\left(\frac{V_{2}}{\sqrt{2 U_{1}}}\right)\right)\right]
\end{aligned}
$$

now:

$$
V_{1}=V-b_{1} \quad V_{2}=V-b_{2}
$$

Then:

$$
P_{\delta t}=100(A+B) \quad(\%)
$$




\section{Annex \\ Procedure for obtaining the fading time distribution from a Log+Log probability of occurrence distribution}

As it was shown in sub-section 2.5.1, starting from (2.22) and for a given threshold $A_{t h}$, the probability of occurrence $P\left(D \mid D \geq D_{0}\right), P(D)$ in simplified notation, can be expressed as the combination of two log-normal functions $[\mathbf{2 2 3}]$ :

$$
P(D)=K_{1} \cdot \operatorname{erfc}\left(\frac{\ln D-\mu_{n, 1}}{\sigma_{n, 1} \sqrt{2}}\right)+K_{2} \cdot \operatorname{erfc}\left(\frac{\ln D-\mu_{n, 2}}{\sigma_{n, 2} \sqrt{2}}\right)
$$

with the constants $K_{1}$ and $K_{2}$ taking the expressions shown in (2.31) and (2.32) respectively. As a reminder, the complementary error function $(e r f c)$ is:

$$
\begin{gathered}
\operatorname{erfc}(x)=1-\operatorname{erf}(x) \\
\operatorname{erfc}(x)=1-\frac{2}{\sqrt{\pi}} \int_{0}^{x} e^{-z^{2}} d z
\end{gathered}
$$

Generalizing:

$$
P(D)=\sum_{\mathrm{i}=1}^{2} K_{i} \cdot \operatorname{erfc}\left(\frac{\ln D-\mu_{\mathrm{n}, \mathrm{i}}}{\sigma_{\mathrm{n}, \mathrm{i}} \sqrt{2}}\right)
$$


The total number of fades $N(D)$ of duration $d$ longer than $D$ can be calculated as $N(D)=$ $N_{\text {tot }} \cdot P(D)$, with $N_{\text {tot }}$ being the total number of fades for the attenuation threshold $A_{t h}$. In order to apply (2.25), in the first place $N(D)$ has to be differentiated with respect to $\ln D$ :

$$
\frac{d N(D)}{d(\ln D)}=\sum_{\mathrm{i}=1}^{2}-\frac{2 \cdot N_{t o t} \cdot K_{i}}{\sigma_{\mathrm{n}, \mathrm{i}} \sqrt{2 \pi}} \cdot \exp \left(-\left(\frac{\ln D-\mu_{\mathrm{n}, \mathrm{i}}}{\sigma_{\mathrm{n}, \mathrm{i}} \sqrt{2}}\right)^{2}\right)
$$

Taking into account that $D=\exp (\ln D)$ and applying $(2.25 \mathrm{~b}),(\mathrm{A} 2.3)$ is obtained:

$$
\frac{d T(D)}{d(\ln D)}=D \frac{d N(D)}{d(\ln D)}=\sum_{\mathrm{i}=1}^{2}-\frac{2 \cdot N_{t o t} \cdot K_{i}}{\sigma_{\mathrm{n}, \mathrm{i}} \sqrt{2 \pi}} \cdot \exp (\ln D) \cdot \exp \left(-\left(\frac{\ln D-\mu_{n, i}}{\sigma_{\mathrm{n}, \mathrm{i}} \sqrt{2}}\right)^{2}\right)
$$

By convenience, this expression can be re-written as:

$$
\frac{d T(D)}{d(\ln D)}=\sum_{\mathrm{i}=1}^{2}-\frac{2 \cdot N_{t o t} \cdot K_{i}}{\sigma_{\mathrm{n}, \mathrm{i}} \sqrt{2 \pi}} \cdot \exp \left(L_{i}\right) \cdot \exp \left(-\left(\frac{\ln D-\mu_{n, i}^{*}}{\sigma_{\mathrm{n}, \mathrm{i}} \sqrt{2}}\right)^{2}\right)
$$

Comparing (A2.3) and (A2.4), the arguments of the exponential functions must be equal:

$$
L_{i}-\left(\frac{\ln D-\mu_{n, i}^{*}}{\sigma_{\mathrm{n}, \mathrm{i}} \sqrt{2}}\right)^{2}=\ln D-\left(\frac{\ln D-\mu_{n, i}}{\sigma_{\mathrm{n}, \mathrm{i}} \sqrt{2}}\right)^{2}
$$

Working with (A2.5) and grouping terms conveniently, it is straightforward to arrive to the expressions for $L_{i}$ and $\mu_{n, i}^{*}$ :

$$
\begin{array}{ll}
\mu_{n, i}^{*}=\mu_{n, i}+\sigma_{n, i}^{2} & i=1,2 \\
L_{i}=\mu_{n, i}+\frac{\sigma_{n, i}^{2}}{2} & i=1,2
\end{array}
$$

Now $T(D)$, can be calculated by integration of (A2.4) and having into consideration that $\operatorname{erfc}(\infty)=0$ and $\operatorname{erfc}(x)=1-\operatorname{erf}(x)$ :

$$
T(D)=\int_{\ln D}^{\infty}\left[\sum_{\mathrm{i}=1}^{2}-\frac{2 \cdot N_{t o t} \cdot K_{i}}{\sigma_{\mathrm{n}, \mathrm{i}} \sqrt{2 \pi}} \cdot \exp \left(L_{i}\right) \cdot \exp \left(-\left(\frac{\ln D-\mu_{n, i}^{*}}{\sigma_{\mathrm{n}, \mathrm{i}} \sqrt{2}}\right)^{2}\right)\right] d \ln D
$$

This integer leads to (A2.7).

$$
T(D)=\int_{\ln D}^{\infty} \frac{d T(D)}{d(\ln D)}=\sum_{\mathrm{i}=1}^{2}-N_{t o t} \cdot K_{i} \cdot \exp \left(L_{i}\right) \cdot\left[\operatorname{erfc}(\infty)-\operatorname{erfc}\left(\frac{\ln D-\mu_{n, i}^{*}}{\sigma_{\mathrm{n}, \mathrm{i}} \sqrt{2}}\right)\right]
$$

which is the same equation as (A2.8) for the case of two distributions.

$$
T(D)=\mathrm{K}_{1} \cdot N_{\text {Tot }} \cdot e^{L_{1}} \cdot \operatorname{erfc}\left(\frac{\ln D-\mu_{n, 1}^{*}}{\sigma_{\mathrm{n}, 1} \sqrt{2}}\right)+\mathrm{K}_{2} \cdot N_{\text {Tot }} \cdot e^{L_{2}} \cdot \operatorname{erfc}\left(\frac{\ln D-\mu_{n, 2}^{*}}{\sigma_{\mathrm{n}, 2} \sqrt{2}}\right)
$$




\section{Annex}

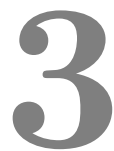

\section{Total Attenuation results}

\section{Total attenuation results}

When the gas attenuation is considered, the following distributions are obtained for the Q-band for each month and year:

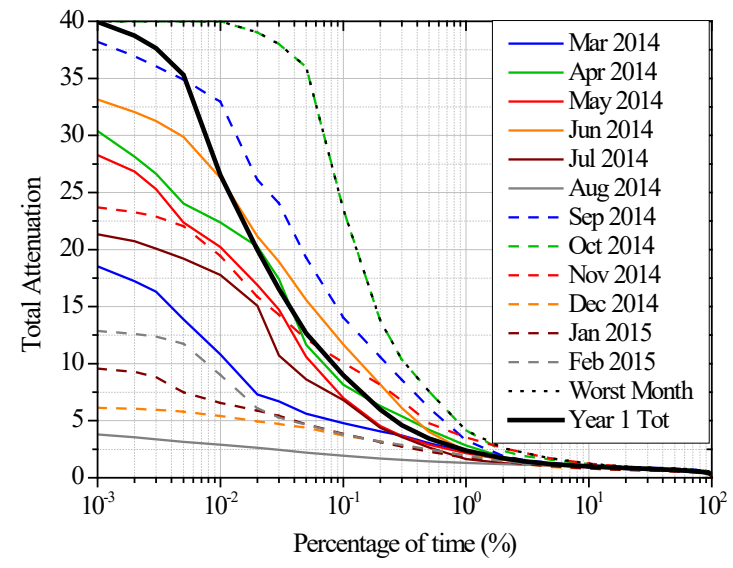

a)

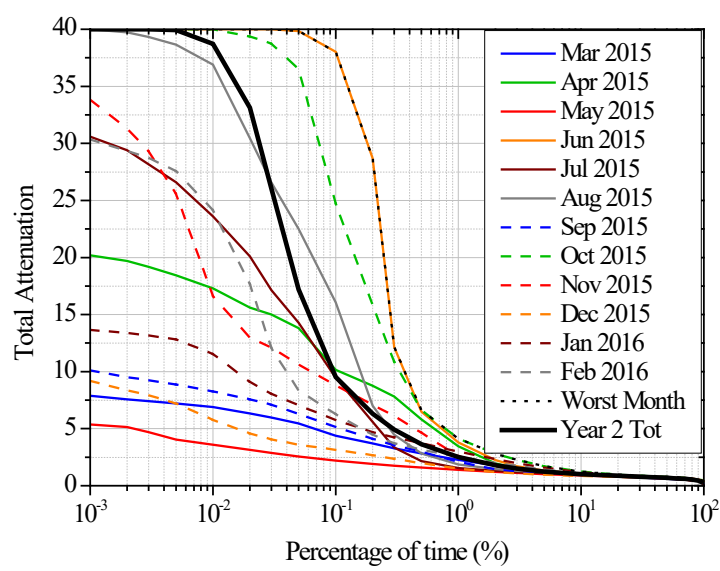

b)

Figure A3.1. Alphasat monthly (including the worst month) and annual Total Attenuation distributions, a) Year 1 and b) Year 2. 


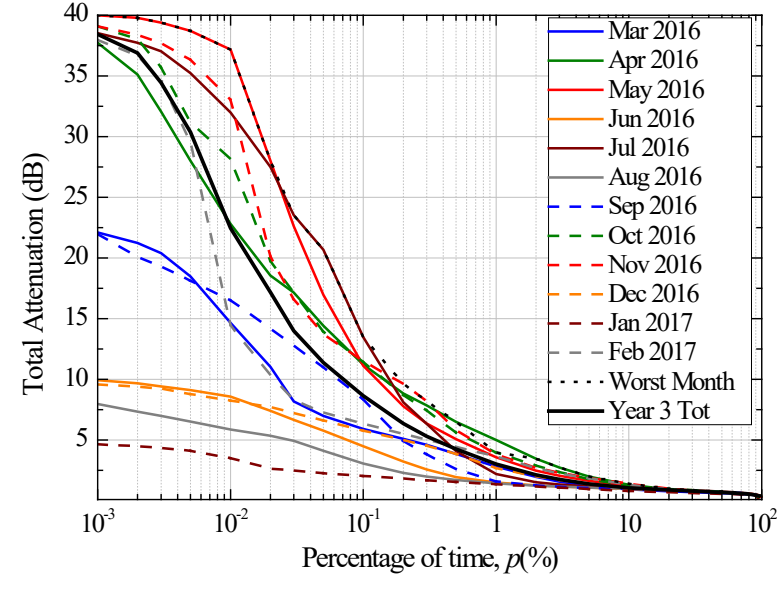

a)

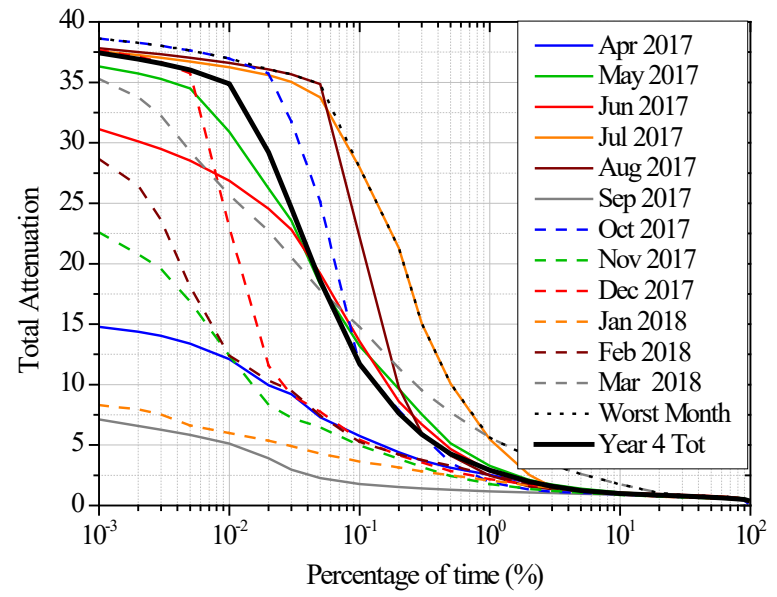

b)

Figure A3.1. Alphasat monthly (including the worst month) and annual Total Attenuation distributions, a) Year 3 and b) Year 4.

If the distributions of Figure A3.1 are compared with their respective counterparts of Figure 5.5, it can be observed that the differences are small and correspond to the gas attenuation contribution. 


\section{Bibliography}

[1] S. Rangan, T.S. Rappaport, and E. Erkip, "Millimeter-Wave Cellular Wireless Networks: Potentials and Challenges," Proceedings of the IEEE (Invited Paper), vol. 102, no. 3, pp. 366-385, March 2014.

[2] HISPASAT. (2018, Feb.) HISPASAT Group Coverage Map. [Online]. https://www.hispasat.com/en/satellite-fleet/coverage-map

[3] SES. (2018, July) SES. [Online]. https://www.ses.com/

[4] Eutelsat. (2018, June) [Online]. https://www.eutelsat.com/en/satellites/find-your-satellite.html

[5] 3GPP. (2018, November) Release 15. [Online]. http://www.3gpp.org/release-15

[6] 3GPP. (2018, November) Release 16. [Online]. http://www.3gpp.org/release-16

[7] P. Kyösti, J. Lehtomäki, J. Medbo, and M. Latva-aho, "Map-Based Channel Model for Evaluation of 5G Wireless Communication Systems," IEEE Transactions on Antennas and Propagation, vol. 65, no. 12, pp. 6491-6504, December 2017.

[8] ITU-R, "Technical feasibility of IMT in bands above 6 GHz," Recommendation ITU-R M.2376-0, 2015.

[9] ITU (WRC), Resolution 238 of the Final Acts of the World Radiocommunication Conference (WRC-15). Geneva, Switzerland, 2015.

[10] METIS, "Intermediate description of the spectrum needs and usage principles," ICT-317669-METIS/ Deliverable 5.1, 2013.

[11] mmMAGIC, "Use case characterization, KPIs and preferred suitable frequency ranges for future 5G systems between $6 \mathrm{GHz}$ and $100 \mathrm{GHz}, "$ ICT-671650-mmMAGIC/ Deliverable D1.1, 2015.

[12] 5G Americas, "White Paper on 5G Spectrum Recommendation," April, 2017.

[13] RSPG, "Strategic Roadmap towards 5G for Europe: Opinion on spectrum related aspects for nextgeneration wireless systems (5G)," Brussels, Belgium, RSPG16-32 FINAL, November, 2016.

[14] MINETAD, "Plan Nacional 5G 2018-2020," Ministerio de Energía, Turismo y Agenda Digital, 2016.

[15] INMARSAT. (2018, Feb) [Online]. https://www.inmarsat.com/press-release/successful-launch-forinmarsat-alphasat-satellite/

[16] ITU-R Rec. P.618-13, "Propagation data and prediction methods required for the design of Earth-space telecommunication systems," ITU Radiocommunication Bureau, Geneva, Switzerland 2017.

[17] J.E. Allnutt, Satellite to Ground Radiowave Propagation, 2nd ed.: The Institution of Engineering and 
Technology, 2011.

[18] P. García del Pino, "Caracterización experimental de la propagación a 50 GHz en trayecto inclinado," Universidad Politécnica de Madrid (UPM), Madrid, PhD Thesis 2003.

[19] José Miguel García Rubia, "Caracterización de la Atenuación por Lluvia en Ondas Milimétricas a partir de Distribuciones Experimentales de Gotal de lluvia," Universidad Politécnica de Madrid (UPM), Madrid, PhD Thesis 2011.

[20] ITU-R Rec. P.676-11, "Attenuation by atmospheric gases," ITU Radiocommunication Bureau, Geneva, Switzerland, 2016.

[21] H.J. Liebe, G.A. Hufford, and M.G. Cotton, "Propagation modeling of moist air and suspender water/ice particles at frequencies below $1000 \mathrm{GHz}, "$ AGARD, Atmospheric Propagation Effects through Natural and Man-made Obscurants for Visible to MM-Wave Radiation, p. 11.

[22] E. Salonen, S. Karhu, S. Uppala, and R. Hyvönen, "Study of improved propagation predictions," Helsinki Univ.Tchnol.Finnish Meteorolog.Inst., Final Rep. ESA/Estec Contract, vol. 9455 (91), pp. 83-87, 1994.

[23] P.W. Rosenkranz, "Water vapor microwave continuum absorption: A comparison of measurements and models," Radio Science, vol. 33, no. 4, pp. 919-928, 1998.

[24] E. Salonen, "Prediction method for oxigen attenuation on slant propagation path for 10-57 GHz," in Antennas and Propagation Society International Symposium, 1991. AP-S. Digest, vol. 3, London, Ontario, Canada, 1991, pp. 1804-1807.

[25] E.T. Salonen, S.M. Uppala, and H.I. Aulamo, "Prediction of water vapour attenuation on a slant path," in Proceedings of the 8th International Conference on Antennas and Propagation, Heriot-Watt University, Edinburgh, UK, Mar. 30 - Apr. 2, 1993, pp. 64-67.

[26] L. Luini and C. G. Riva, "A Simplified Model to Predict Oxygen Attenuation on Earth-Space Links," IEEE Transactions on Antennas and Propagation, vol. 65, no. 12, pp. 7217-7223, Dec. 2017.

[27] ITU-R Rec. P.836-6, "Water vapour: surface density and total columnar content," ITU Radiocommunication Bureau, Geneva, Switzerland, 2017.

[28] ITU-R Rec. P.1510-1, "Mean surface temperature," ITU Radiocommunication Bureau, Geneva, Switzerland, 2017.

[29] WMO, Guide to Meteorological Instrument and Methods of Observation WMO No. 8, 7th ed.: Word Meteorological Organization, 2008.

[30] G. Brussaard and P.A. Watson, Atmospheric Modelling and Millimetre Wave Propagation. London: Ed. Chapman \& Hall, 1995.

[31] R.R. Rogers and M.K. Yau, A short Course in Cloud Physics.: Elsevier, 1996.

[32] H. Pruppacher and J. Klentt, Microphysics of clouds and precipitation. The Netherlands: Atmospheric and Oceanographic Sciences Library Vol. 18 Kluwer Academic Publishers, 1997.

[33] Pao K. Wang, Physics and Dynamics of Clouds and Precipitation. United Kingdom: Cambridge University Press, 2013.

[34] R.A. Houze Jr., Cloud dynamics, Volume 104 of International Geophysics, 2nd ed.: Academic Press, 2014.

[35] V.I. Khvorostyanov and J.A. Curry, Thermodynamics, kinetics, and microphysics of clouds. USA: Cambridge University Press, 2014.

[36] National Oceanic and Atmospheric Administration. (2017, July) National Weather Service. [Online]. http://www.srh.noaa.gov/srh/jetstream/clouds/cloudwise/types.html

[37] M. Decker, E. Westwater, and F. Guiraud, "Experimental evaluation of ground-based microwave 
radiometric sensing of atmospheric temperature and water vapor profiles," Journal of Applied Meteorology, vol. 17, no. 12, pp. 1788-1795, 1975.

[38] E. Salonen and S. Uppala, "New prediction method of cloud attenuation," Electronics Letters, vol. 27, no. 12, pp. 1106-1108, 1991.

[39] V. Mattioli, P. Basili, S. Bonafoni, P. Ciotti, and E.R. Westwater, "Analysis and improvement of cloud models for propagation studies," Radio Science, vol. 44, 2009.

[40] E.E. Altshuler and R.A. Marr, "Cloud Attenuation at Millimeter Wavelengths," IEEE Transaction on Antennas and Propagation, vol. 37, no. 11, pp. 1473-1459, Nov. 1987.

[41] F. Dintelmann and G. Ortgies, "Semiempirical model for cloud attenuation prediction," Electronics Letters, vol. 25, no. 22, pp. 1487-1488, Oct. 1989.

[42] L. Luini and C. Capsoni, "Efficient Calculation of Coud Attenuation for Earth-Space Applications," IEEE Antennas and Wireless Propagation Letters, vol. 13, pp. 1136-1139, 2014.

[43] A. Dissanayake, J. Allnutt, and F. Haidara, "A prediction model that combines rain attenuation and other propagation impairments along Earth-space paths," IEEE Transactions on Antennas and Propagation, vol. 45, no. 10, pp. 1546-1558, Oct. 1997.

[44] A. Dissanayake, J. Allnut, and J. Haidara, "Cloud attenuation modelling for SHF and EHF applications," International Journal of Satellite Communications and Networking, vol. 19, pp. 335-345, 2001.

[45] ITU-R Rec. P.840-6, "Attenuation due to clouds and fog," ITU Radiocommunication Bureau, Geneva, Switzerland, 2013.

[46] C. Riva, "Spatial characteristics of propagation parameters: a review," in COST 2801 St International Workshop, Malvern, UK, 2002.

[47] C. Capsoni, C. Magistroni, A. Paraboni, A Pawlina, and F. Fedi, "Data and theory for a new model of the horizontal structure of rain cells for propagation applications," Radio Science, vol. 22, no. 3, pp. 395404, 1987.

[48] L. Féral, H. Sauvageot, L. Castanet, and J. Lemorton, "HYCELL- A new hybrid model of the rain horizontal distriburion for propagation studies: 1. Modeling of the rain cell," Radio Science, vol. 38, no. 3, p. 1056, 2003.

[49] L. Luini and C. Capsoni, "MultiEXCELL: a new rainfall model for the analysis of the millimetre wave propagation through the atmosphere," in 3th European Conference on Antenna and Propagation (EuCAP), Berlin, Germany, 2009, pp. 1946-1950.

[50] ITU-R Rec. P.839-4, "Rain height model for prediction methods," ITU Radiocommunication Bureou, Geneva, Switzerland, 2013.

[51] J.O Laws and D.A. Parsons, "The relation of raindrop size to intensity," Trans. Amer. Geophys. Union, pp. 452-460, 1943.

[52] J. Joss, J.C. Thams, and A. Waldvogel, "The variation of rain drop size distribution at Locarno," Issue 64 of Wissenschaftliche Mitteilungen / Eidgenössische Kommission zum Studium der Hagelbildung und der Hagelabwehr, pp. 369-373, 1970.

[53] J.S. Marshall and W.McK. Palmer, "The distribution of rain drops with size," Journal of Meteorology, vol. 5, pp. 165-166, 1948.

[54] D. Atlas and C.W. Ulbrich, "The physic basis for attenuation-rainfall relationships and the measurement of rain parameters by combined attenuation and radar methods," J. Research. Atmos., vol. 8, no. 1-2, pp. $275-298$.

[55] M. Sekine and G. Lind, "Rain attenuation of centimeter, millimeter and submillimeter radio waves," in 12th European Mocrowave Conference, Helsinki, 1982, pp. 584-589. 
[56] J.M. Garcia-Rubia, J.M. Riera, P. Garcia-de- Pino, and A. Benarroch, "Attenuation Measurements and Propagation Modeling in the W-Band," IEEE Transactions on Antennas and Propagation, vol. 61, no. 4, pp. 1860-1867, Apr. 2013.

[57] ITU-R Rec. P.837-6, "Characteristics of precipitation for propagation modelling," ITU Radiocommunication Bureau, Geneva, Switzerland, 2012.

[58] ITU-R Rec. P.837-7, "Characteristics of precipitation for propagation modelling," ITU Radiocommunication Bureau, Geneva, Switzerland, 2017.

[59] ITU-R Study Group 3, "Description of the method adopted for the update of Rec. ITU-R P. 837-5, Annex 3," SG3 Report Document 3J/TEMP/XX-E, 2011.

[60] ITU-R Rec. P.1144-7, "Guide to the application of the propagation methods of Radiocommunication Study Group 3," ITU Radiocommunication Bureau, Geneva, Switzerland, 2015.

[61] L. Luini and C. Capsoni, "A Unified Model for the Prediction of Spatial and Temporal Rainfall Rate Statistics," IEEE Transactions on Antennas and Propagation, vol. 61, no. 10, pp. 5249-5254, Oct. 2013.

[62] ITU-R Study Group 3, "Proposed revision of Recommendation ITU-R P.837-6," Input Document 3J/84E, March 2017.

[63] COST, "Radiowave Propagation Modelling for SatCOm Services at Ku-Band and Above," Cost Action 255 Final Report, ESA Publications Division, Noordwijk, The Netherlands, 2002.

[64] ITU-R Rec. P.838-3, "Specific attenuation model for rain for use in prediction methods," ITU Radiocommunication Bureau, Geneva, Switzerland, 2005.

[65] COST 255, "COST Action 255 Final Report: Radiowave Propagation for SatCom Services at Ku-Band," ESA, Final Report 2002.

[66] M.S. Assis and C.M. Einloft, "A simple method for estimating rain attenuation distribution," Annals of Telecommunications, vol. 32, no. 11, pp. 478-480, 1977.

[67] R.K. Flavin, "Satellite link rain attenuation in Brisbane and a proposed new model for Australia," Telstra Research Laboratories, Report N. 8375, 1996.

[68] CCIR Document 5C/52 (Brazil), "Slant-path Attenuation Prediction Method Based on the Complete Point Rainfall Rate Distribution," ITU, Geneva, Switzarland, 1992.

[69] G.H. Bryant, I. Adimula, C. Riva, and G. Brussaard, "Rain attenuation statistics from rain cell diameters and heigths," International Journal of Satellite Communications and Networking, vol. 19, no. 3, pp. 263-283, 2001.

[70] R.K. Crane, "Prediction of attenuation by rain," IEEE Transactions on Communications, vol. 28, no. 9, pp. 1717-1733, 1980 .

[71] R.K. Crane, "A two-component rain model for the prediction of attenuation statistics," Radio Science, vol. 17, pp. 1371-1387.

[72] J.A. Garcia-Lopez, J.M. Hernando, and J.M. Selga, "Simple rain attenuation prediction method for satellite radio links," IEEE Transactions on Antennas and Propagation, vol. 36, pp. 444-448, 1988.

[73] M. Yamada, Y. Karasawa, M. Yasunaga, and B. Arbesser-Rastburg, "An improved prediction method for rain attenuation in satellite communictions operating at 10-20 GHz," Radio Science, vol. 22, pp. 1053 $1062,1987$.

[74] M.J. Leitao and P.A. Watson, "Method for prediction of attenuation on earth-space links based on radar measurements of the physical structure of rainfall," IEE Proceedings F Communications, Radar and Signal Processing, vol. 133, no. 4, pp. 429-440, 1986.

[75] E. Matricciani, "Rain attenuation predicted with a two-layer rain model," Eur. Trans. Telecommun., vol. 
2, no. 6, pp. 715-727, 1991.

[76] A.D. Panagopoulos, D.M. Arapoglou, J.D. Kanellopoulos, and P.G. Cottis, "Long-term rain attenuation probability and site diversity gain prediction formulas," IEEE Transactions on Antennas and Propagation, vol. 53, no. 7, pp. 2307-2313, 2005.

[77] W.L. Stutzman and W.K. Dishman, "A simple model for the estimation of rain-induced attenuation along earth-space paths at milimeter wavelengths," Radio Science, vol. 17, no. 6, pp. 1465-1476, 1982.

[78] W.L. Stutzman and K.M. Yon, "A simple rain attenuation model for earth-space radio links operating at 10-35 GHz," Radio Science, vol. 21, pp. 65-72, 1986.

[79] L. Svjatogor, "Prostranstvennaia korelacia vypadenjija dozdjej vdol zemnoj poverchnostji," Interkosmos Symposium Theme 5 of the Established Telecommunication Working Group, 1985.

[80] Ch-S. Lu et al., "A New Rain Attenuation Prediction Model for the Earth-Space Links," IEEE Transactions on Antennas and Propagation, vol. 66, no. 10, pp. 5432-5442, 2018.

[81] E. Matricciani, "Physical-mathematical model of the dynamics of rain attenuation based on rain rate time series and a two-layer vertical structure of precipitation," Radio Science, vol. 31, no. 2, pp. 281-295, 1996.

[82] M.-S. Alouini and P.G. Steffes, "Impact of tropospheric scintillation on direct-sequence spread-spectrum satellite communication networks," in Proceedings of ISSE'95 - International Symposium on Signals, Systems and Electronics, San Francisco, USA, 1995, pp. 67-70.

[83] M.M.J.L. van de Kamp, C. Riva, J.K. Tervonen, and E.T. Salonen, "Frequency Dependence of Amplitude Scintillation," IEEE Transactions on Antennas and Propagation, vol. 47, no. 1, pp. 77-85, 1999.

[84] O.P. Banjo and E. Vilar, "The dependence of slant path amplitude scintillations on various meteorological parameters," in International Conference on Antennas and Propagation, 5th., York, England, 1987, pp. 277-280.

[85] J. Tervonen, M.M.J.L. van de Kamp, and E.T. Salonen, "Prediction Model for the Diurnal Behavior of the Tropospheric Scintillation Variance," IEEE Transactions on Antennas and Propagation, vol. 46, no. 9, pp. 1372-1377, Sep. 46.

[86] V.I. Tatarskii, Wave Propagation in a Turbulent Medium. New York, EEUU: McGraw Hill, 1961.

[87] T.J. Moulsley and E. Vilar, "Experimental and Theoretical Statistics of Microwave Amplitude Scintillations," IEEE Transactions on Antennas and Propagation, vol. 30, no. 6, pp. 1099-1106, 1982.

[88] H. Vasseur, "Prediction of Tropospheric Scintillation on Satellite links from Radiosonde Data," IEEE Transactions on Antennas and Propagation, vol. 47, no. 2, pp. 293-301, feb. 1999.

[89] R.K. Crane and D.W. Blood, "Handbook for the estimation of microwave propagation effects," NASA GSFC , Greenbelt, MA, NASA Contract NAS5-25341 Technical Report n. 1, Doc. 7376-TR1, 1979.

[90] J. Haddon and E. Vilar, "Scattering Induced Microwave Scintillation from Clear Air and Rain on EarthSpace Paths and the Influence of the Antenna Aperture," IEEE Transactions on Antennas and Propagation, vol. AP-34, no. 5, pp. 646-657, May 1986.

[91] Y. Karasawa, M. Yamada, and J. Allnutt, "A New Prediction Method for Tropospheric Scintillation on Earth-Space Paths," IEEE Transactions on Antennas and Propagation, vol. 36, no. 11, pp. 1608-1614, Nov. 1988.

[92] I.E. Otung, "Prediction of tropospheric amplitude scintillation on a satellitelink," IEEE Transactions on Antennas and Propagation, vol. 44, no. 12, pp. 1600-1608, dec. 1996.

[93] C.N. Kassianides and I.E. Otung, "Dynamic Model of tropospheric scintillation on Earth-space paths," IEE Proc.-Microw. Antennas Propag., vol. 150, no. 2, pp. 97-104, Apr. 2003. 
[94] I.E. Otung and A. Savvaris, "Estimating tropospheric scintillation intensity on earth-space propagation paths," Electronics Letters, vol. 42, no. 7, pp. 381-382, 2006.

[95] M.M.J.L. van de Kamp, J.K. Tervonen, E.T. Salonen, and P. Baptista, "Improved models for long-term prediction of tropospheric scintillation on slant paths," IEEE Transactions on Antennas and Propagation, vol. 47, no. 2, pp. 249-260, 1999.

[96] F.S. Marzano and G. d'Auria, "Model-based prediction of amplitude scintillation variance due to clearair tropospheric turbulence on Earth-satellite microwave links," IEEE Transactions on Antennas and Propagation, vol. 46, no. 10, pp. 1508-1518, Oct. 1998.

[97] G. Peeters, F.S. Marzano, G. d'Auria, C. Riva, and D. Vanhoenacker-Janvier, "Evaluation of Statistical Models for clear-air Scintillation Prediction Using Olympus Satellite Measurements," International Journal of Satellite Communications, vol. 15, pp. 73-88, 1997.

[98] G. Ortgies, "Prediction of slant-path amplitude scintillation on a satellite link," in Proceedings of International Symposium on Radio Propagation, Pekin, China, 1993, pp. 218-221.

[99] E. Matricciani and C. Riva, "18.7 GHz tropospheric scintillation and simultaneous rain attenuation measured at Spino d'Adda and Darmstadt with Italsat," Radio Science, vol. 43, 2008.

[100] P. Garcia-del-Pino, J.M. Riera, and A. Benarroch, "Tropospheric Scintillation With Concurrent Rain Attenuation at $50 \mathrm{GHz}$ in Madrid," IEEE Transaction on Antennas and Propagation, vol. 60, no. 3, pp. 1578-1583, Mar. 2012.

[101] ITU-R Rec. P.1623-1, "Prediction method of fade dynamics on Earth-space paths," ITU Radiocommunication Bureau, Geneva, Switzerland, 2005.

[102] COST 205, "Influence of the atmosphere on radiopropagation on satellite earth paths at frequencies above $10 \mathrm{GHz}$," Commission of the European Communities, Brussels, ISBN 92-825-5412-0, 1985.

[103] J.S. Mandeep, "Fade duration statistics for Ku-band satellite link," Advances in Space Research, vol. 52, pp. 445-450, 2014.

[104] M. Cheffena and C. Amaya, "Prediction Model of Fade Duration Statistics for Satellite Links Between 10-50 GHz," IEEE Antennas and Wireless Propagation Letters, vol. 7, pp. 260-263, 2008.

[105] C. Amaya and T. Nguyen, "Fade Duration and Fade Slope Statistics Derived from Long-Termn Anik-F2 Satellite Beacon Measurements in Ottawa-Canada," General Assembly and Scientific Symposium, 2011 XXXth URSI, Istanbul, pp. 1-4, 2011.

[106] P. Garcia-del-Pino, J.M. Riera, and A. Benarroch, "Fade and interfade duration statistics on an Earthspace link at $50 \mathrm{GHz}$, IET Microwave, Antennas $\&$ Propagation, vol. 5, no. 7, pp. 790-794, 2011.

[107] United States of America, "Working document toward a revision to the fade duration prediction method in Recommendation ITU-R P. 1623," ITU-R SG-3, Geneva, Switzerland, ITU Document 3M/36-E, 2003.

[108] A. Paraboni and C. Riva, "A new method for the prediction of fade duration statistics in satellite links beyond $10 \mathrm{GHz}, "$ International Journal of Satellite Communications, vol. 12, pp. 387-394, 1994.

[109] L.E. Bråten, C. Amaya, and D.V. Rogers, "Fade durations on Earth-Space links: dependence on path and climatic parameters," in Proc. of URSI Commission F Meeting on Climatic Parameters in Radiowave Propagation Prediction (CLIMPARA 2001), Budapest, Hungary, 2001, pp. 107-111.

[110] R. Lekkla, K. McCormick, and D. Rogers, "12-GHz fade duration statistics on Earth-space paths in South-East Asia," in Proc. URSI-CLIMPARA 1998, Ottawa, Canada, pp. 167-170.

[111] C. Amaya, "Analysis of fade duration along Earth-space paths in the tropics," in The Second European Conference on Antennas and Propagation, EuCAP 2007, Edinburgh, United Kingdom, 2007, pp. 1-8.

[112] ESA, "Characterisation and modeling of propagation effects in the 20-50 GHz band," Final Report of ESA/ESTEC Contract Nº17760/03/NL/JA, 2006. 
[113] United States of America, "Working document toward a revision of recommendation ITU-R P. 1623 including a new inter-fade interval prediction method," ITU-R SG-3, Geneva, Switzerland, Document 3J/44-E 3M/59-E, 2004.

[114] F. Jorge, C. Riva, and A. Rocha, "Interfade and Inter-Event Interval: a Time-Based Classification and Modelling," in EuCAP 2016, Davos, Switzerland, 2016.

[115] J.P.V. Poilares Baptista and P.G. Davies, Eds., OPEX; Volume 1: Reference Book on Attenuation Measurement and Prediction. Noordwijk, Netherlands, 1994.

[116] E. Matricciani, "Rate of change of signal attenuation from Sirio at $11.6 \mathrm{GHz}, "$ Electronics Letters, vol. 17, no. 3, pp. 139-141, Feb. 1981.

[117] F.F. Franklin, K. Fujisaki, and M. Tateiba, "Fade Dynamics on Earth-Space Paths at Ku-Band in Fukuoka, Japan Fade-Slope Evaluation, Comparison, and Model," IEEE Antennas and Wireless Propagation Letters, vol. 5, pp. 80-83, 2006.

[118] P. Garcia-del-Pino, J.M. Riera, and A. Benarroch, "Dynamic characteristics of fading on a $50 \mathrm{GHz}$ slant path," in First European Conference on Antennas and Propagation, EuCAP 2006, Nice, 2006, pp. 1-6.

[119] B. Nelson and W.L. Stutzman, "Fade slope on 10 to $30 \mathrm{GHz}$ earth-space communication links measurement and modelling," IEE Proc-Microw. Antennas Propag, vol. 14, no. 4, pp. 353-357, Aug. 1996.

[120] M.M.J.L. van de Kamp, "Climatic radiowave propagation models for the design of satellite communication systems," Technische Universiteit Eindhoven, Eindhoven, PhD dissertation 1999.

[121] M,M.J.L. van de Kamp, "Statistical analysis of rain fade slope," IEEE Transactions on Antennas and Propagation, vol. 51, no. 8, pp. 1750-1759, Aug. 2003.

[122] H. Dao, Md.R. Islam, and K.A.S. Al-Khateeb, "Rain Fade Slope Model in Satellite Path Based on Data Measured in Heavy Rain Zone," IEEE Antennas and Wireless Propagation Letters, vol. 12, pp. 50-53, January 2013.

[123] D. Das and A. Maitra, "Fade-Slope Model for Rain Attenuation Prediction in Tropical Region," IEEE Geoscience and Remote Sensing Letters, vol. 13, no. 6, pp. 777-781, June 2016.

[124] A. Panagopoulos, P-M.M. Arapoglou, and P.G. Cottis, "Satellite Communications at Ku, Ka and V bands: Propagation Impairments and Mitigation Techniques," IEEE Communications Surveys $\mathcal{G}$ Tutorial, vol. 6, no. 3, pp. 2-14, Third Quarter 2004.

[125] D.B. Hodges, "Path diversity for reception of satellite signals," Journal de Recherches Atmospheriques, vol. 8, pp. 443-449, June 1974.

[126] ITU-R Rec. P.1853-1, "Tropospheric attenuation time series synthesis," ITU Radiocommunication Bureau, Geneva, Switzerland 2012.

[127] E. Matricciani, "Probability distributions of rain attenuation obtainable with linear combining techniques in space-to-Earth links using time diversity," International Journal of Satellite Communications and Networking, 2017.

[128] V. Fabbro, L. Castanet, S. Croce, and C. Riva, "Characterization and modelling of time diversity statistics for satellite communications from 12 to $50 \mathrm{GHz}, "$ International Journal of Satellite Communications, vol. 27, no. 2, pp. 87-101, 2009.

[129] A.F. Ismail and P.A. Watson, "Characteristics of fading and fade countermeasures on a satellite-Earth link operating in an equatorial climate, with reference to broadcast applications," IEE Proc.-Microw. Antennas Propag., vol. 147, no. 5, 200.

[130] E. Matricciani, "Time diversity as a rain attenuation countermeasure in satellite links in the 10-100 GHz frequency bands," in EuCAP 2006, Nice, 2006, pp. 6-10. 
[131] V. Fabbro and L. Castanet, "Characterization and Modelling of Time Diversity in 20-50 GHz Band," in EuCAP 2006, Nice, France, 2006.

[132] ESA, "Characterisation and modelling of time diversity statistics for satellite comunications from 12 to $50 \mathrm{GHz}, "$ ITU-R WPs, Boulder, USA, input document 3J/48-3M/47, May 2008.

[133] A. Paraboni and F. Barbaliscia, "Multiple site attenuation prediction models based on the rainfall structures (meso- or synoptic-scales) for advanced TLC or broadcasting systems'," in XXVIIth URSI general asambly, Maastricht, Netherlands, 2002.

[134] M. Luglio, R. Mancini, C. Riva, A. Paraboni, and F. Barbaliscia, "Large-scale site diversity for satellite communication networks," International Journal of Satellite Communications and Networking, vol. 20, no. 4, pp. 251-260, Jul. 2002.

[135] C. Capsoni, M. Ferrari, A. Paraboni, and C. Riva, "Spatial-instantaneous versus point-long term cdf of rain rate," ESA workshop on Earth-space propagation, Noordwijk, Netherlands, 2005.

[136] P-D.M. Arapoglou, A.D. Panagopoulos, and P.G. Cottis, "An Analytical Prediction Model of Time Diversity Performance for Earth-Space Fade Mitigation," International Journal of Antennas and Propagation, 2008.

[137] Greece, "Information document on the prediction of time diversity in terrestrial LOS links operating above $10 \mathrm{GHz}, "$ NTUA, Greece, input document 3K/68-3M/72, May 2009.

[138] C. Kourogiorgas, A.D. Panagopoulos, S.N. Livieratos, and G.E. Chatzarakis, "On the Outage Probability Prediction of Time Diversity Scheme in Broadband Satellite Communication Systems," Progress In Electromagnetics Research C, vol. 44, pp. 175-184, 2013.

[139] T. Maseng and P.M. Bakken, "A Stochastic Dynamic Model of Rain Attenuation," IEEE Transactions on Communications, vol. 29, no. 5, pp. 660-669, May 1981.

[140] ITU-R Rec. P.1057-4, "Probability distributions relevant to radiowave propagation modelling," ITU Radiocommunication Bureau, Geneva, Switzerland, 2015.

[141] X. Boulanger, "Development of space-time correlated rain attenuation time series synthesizer and site diversity modelling," internal funding RT 1/25373 DEMR 2016.

[142] X. Boulanger et al., "A Rain Attenuation Time-Series Synthesizer Based on a Dirac and Lognormal Distribution," IEEE Transactions on Antennas and Propagation, vol. 61, no. 3, pp. 1396 - 1406, March 2013.

[143] J. Kanellopoulos and S. Ventouras, "Prediction of the outage performance of an unbalanced orbital diversity Earth-Space system," AFRICON '92 Proceedings., 3rd AFRICON Conference, 1992.

[144] E. Matricciani, "Orbital Diversity in Resource-Shared Satellite Communication Systems Above 10 GHz," IEEE Journal on Selected Areas in Communications, vol. SAC-5, no. 4, pp. 714-722, May 1987.

[145] C. Capsoni, E. Matricciani, and M. Mauri, "SIRIO-OTS 12 GHz orbital diversity experiment at Fucino," IEEE Transactions on Antennas and Propagation, vol. 38, no. 6, pp. 777-782, June 1990.

[146] E. Matricciani and M. Mauri, "Italsat-Olympus 20-GHz orbital diversity experiment at Spino d'Adda," IEEE Transactions on Antennas and Propagation, vol. 43, no. 1, pp. 105-108, January 1995.

[147] J. Flávio, F. Perez-Fontan, S. Mota, and A. Rocha, "Joint Results of the Aveiro and Vigo Alphasat Propagation Campaigns," in 11th European Conference on Antennas and Propagation(EUCAP), Paris, France, 2017, pp. 2381-2384.

[148] E. Matricciani, "An orbital diversity model for earth to space links under rain and comparisons with site diversity," Radio Science, vol. 18, no. 4, pp. 583-588, July-August 1983.

[149] J.D. Kanellopoulos and A. Reppas, "Prediction of the outage performance of an orbital diversity EarthSpace communication system," in Proceedings of the 6th Mediterranean Electrotechnical Conference, 
Lubljiana, Slonevia, 1991.

[150] J. Kanellopoulos and S. Ventouras, "Prediction of the Outage Performance of an Unbalanced Orbital Diversity Earth-Space System (Log-Normal Case)," Transactions on Emerging Telecommunications Technologies, vol. 4, no. 4, pp. 415-421, July 1993.

[151] J.D. Kanellopoulos, S. Ventouras, and S. Koukoulas, "Prediction of the Outage Performance of an Unbalanced Orbital Diversity Diversity Earth- Space System (GAMMA Case)," Journal of Electromagnetic Waves and Applications, vol. 7, no. 9, pp. 1257-1270, 1993.

[152] A.D. Panagopoulos and J.D. Kanellopoulos, "On the rain attenuation dynamics: spatial-temporal analysis of rainfall rate and fade duration statistics," International Journal of Satellite Communications and Networking, vol. 21, no. 6, pp. 595-611, July 2003.

[153] N. Jeannin, X. Boulanger, L. Féral, L. Castanet, and F. Lacoste, "Inter-annual variability, risk and confidence intervals associated with propagation statistics. Part I: theory of estimation," International Journal of Satellite Communication and Networking, vol. 32, pp. 407-421, 2014.

[154] ITU-R Rec. P.678-3, "Characterization of the variability of propagation phenomena and estimation of the risk associated with propagation margin," ITU Radiocommunication Bureau, Geneva, Switzerland, 2015.

[155] A. Burgueño, J. Austin, E. Vilar, and M. Puigcerver, "Analysis of Moderate and Intense Rainfall Rates Continuously Recorded Over Half a Century and Influence on Microwave Communications Planning and Rain-Rate Data Acquisition," IEEE Transactions on Communications, vol. COM-35, no. 4, pp. 382-395, April 1987.

[156] J.P.V. Poilares Baptista, Z.W. Zhang, and N.J. McEwan, "Stability of rain rate cumulative distribution," Electronics Letters, vol. 22, no. 7, pp. 350-352, March 1986.

[157] J. Goldhirsh, "Slant Path Fade and Rain-Rate Statistics Associated with the COMSTAR Beacon at 28,56 GHz for Wallops Island, Virginia over a Three-Year Period," IEEE Transactions on Antennas and Propagation, vol. 30, no. 2, pp. 191-198, March 1982.

[158] C. Riva, "Seasonal and diurnal variations of total attenuation measured with the ITALSAT satellite at Spino d'Adda at 18.7,39.6 and $49.5 \mathrm{GHz}$," International Journal of Satellite Communications and Networking, vol. 22, no. 4, pp. 449-476, July 2004.

[159] J.M. Garcia-Rubia, J.M. Riera, P. Garcia-del-Pino, G.A. Siles, and A. Benarroch, "Experimental assessment of slant-path rain attenuation variability in the Ka-band," International Journal of Satellite Communications and Networking, vol. 34, no. 2, pp. 155-170, March/April 2015.

[160] J.M. Garcia-Rubia, J.M. Riera, P. Garcia-del-Pino, G.A. Siles, and A. Benarroch, "Fade Dynamics Variability in a Long-Term Slant-Path Ka-Band Experiment," in 2015 9th European Conference on Antennas and Propagation (EuCAP), Lisbon, 2015, pp. 1-5.

[161] J.M. Garcia-Rubia, J.M. Riera, P. Garcia-del-Pino, D. Pimienta-del-Valle, and G.A. Siles, "Fade and Inter-Fade Duration Characteristics in a Slant-Path Ka-Band Link," IEEE Transactions on Antennas and Propagation, vol. 65, no. 12, pp. 7198-7206, December 2017.

[162] ITU-R. (2017) [Online]. http://www.itu.int/en/ITU-R/study-groups/rsg5/rwp5d/imt2020/Pages/default.aspx

[163] A. Aragón-Zabala, Indoor Wireless Communications: From Theory to Implementation.: John Wiley \& Sons Ltd., 2017.

[164] T. Rappaport, R.W. Hearh Jr., R.C. Daniels, and J.N. Murdock, Millimeter Wave Wireless Communications. Westford, Massachusetts, EEUU: Prentice Hall, 2014.

[165] ETSI, "5G; Study on Scenarios and Requirements for Next Genaration Access Technologies (3 GPP TR 38.913 version 14.2.0 Release 14)," Technical Report ETSI TR 138913 v14.2.0, 2017. 
[166] METIS. (2017, June) METIS 2020. [Online]. https://www.metis2020.com/

[167] 5G PPP. (2017, November) 5G PPP. [Online]. https://5g-ppp.eu/mmmagic/

[168] ETSI. (2017, November) ETSI. [Online]. http://www.etsi.org/news-events/news/866-2015-01-press-newetsi-group-on-millimetre-wave-transm

[169] NYU Tandon School of Engineering. (2017, November) NYU Wireless. [Online]. http://wireless.engineering.nyu.edu/

[170] NIST. (2017, November) NIST. [Online]. https://www.nist.gov/programs-projects/metrology-supportwireless-innovation

[171] 5GPPP. (2018, April) mmMAGIC. [Online]. https://5g-mmmagic.eu/results/

[172] METIS. (2018, April) [Online]. https://www.metis2020.com/documents/index.html

[173] 5GCM. (2017) 4th International Workshop on 5G/5G+ Communications in Higher Frequency Bands. [Online]. http://www.5gworkshops.com/5GCM.html

[174] T.S. Rappaport et al., "Overview of Millimeter Wave Communications for Fifth-Generation (5G) Wireless Networks - With a Focus on Propagation Models," IEEE Transactions on Antennas and Propagation, vol. 65, no. 12, pp. 6213-6230, December 2017.

[175] IEEE Antennas and Propagation Society, "Special Issue on Antennas and Propagation Aspects of 5G Communications," in IEEE Transactions on Antennas and Propagation Vol. 65 No. 12, D. Erricolo, Ed., December, 2017.

[176] L. Raschkowski, P. Kyösti, K. Kusume, and T. Jämsä (Ed.), "METIS Channel Models, deliverable 1.4 v3," METIS Proyect Technical Report ICT-317669. [Online]. Available: https://www.metis2020.com/, 2015 .

[177] P. Kyösti, J. Meinilä, L. Hentilä, X. Zhao, and et al., "WINNER II channel models," European Commission, Tech. Rep. D1.1.2, Sept. 2007.

[178] ITU-R Report M.2135-1, "Guidelines for the evaluation of radio interface technologies for IMTAdvanced," ITU Radiocommunication Bureau, Geneva, Switzerland, 2009.

[179] WP5: Propagation, Antennas and Multi-Antenna Technniques, "D5.1: Channel Modeling and Characterization," Millimetre-Wave Evolution for Backhaul and Access(MiWEBA) 2014.

[180] Aalto University et al., "5G Channel Model for bands up to $100 \mathrm{GHz}$," in 4th International Workshop on 5G/5G+ Communications in Higher Frequency Bands (5GCHFB), 2017.

[181] M. Peter, K. Sakaguchi, and et al., "Measurement Campaigns and Initial Channel Models for Preferred Suitable Frequency Ranges," 5GPPP, mmMAGIC Deliverable D2.1 ICT-671650, 2016.

[182] G. MacCartney Jr., T.S. Rappaport, S. Shu, and D. Sijla, "Indoor Office Wideband Millimeter-Wave Propagation Measurements and Channel Models at 28 and $73 \mathrm{GHz}$ for Ultra-Dense 5G Wireless Networks," Invited paper in IEEE Access, vol. 3, pp. 2388-2424, 2015.

[183] S. Sun et al., "Investigation of Prediction Accuracy, Sensitivity, and Parameter Stability of Large-Sacale Propagation Path Loss Models for 5 G Wireless Communications," IEEE Transactions on Vehicular Technology, vol. 65, no. 5, pp. 2843-2859, May 2016.

[184] S. Salous and Y. Gao, "Wideband measurements in indoor and outdoor environments in the $30 \mathrm{GHz}$ and $60 \mathrm{GHz}$ bands," in 2016 10th European Conference on Antennas and Propagation (EuCAP 2016), Paris, France, 2016.

[185] S. Piersanti, L.A. Annoni, and D. Cassioli, "Millimeter Waves Channel Measurements and Path Loss Models," in IEEE ICC 2012 - Wireless Communications Symposium, Otawa, Canada, 2012, pp. 45524556 . 
[186] ITU-R Rep. P.2406-0, "Studies for short-path propagation data and models for terrestrial radiocommunication systems in the frequency range $6 \mathrm{GHz}$ to $100 \mathrm{GHz}$," ITU Radiocommunication Bureau, Geneva, Switzerland, 2016.

[187] ITU-R Rep. P.2346-2, "Compilation of measurement data relating to building entry loss," ITU Radiocommunication Bureau, Geneva, Switzerland 2017.

[188] 3GPP. "Technical specification group radio access network, channel model for frequency spectrum above $6 \mathrm{GHz}, "$ TR 38.900 v 14.3.1, Jul. 2017. [Online]. https://portal.3gpp.org/desktopmodules/Specifications/SpecificationDetails.aspx?specificationId=2991

[189] 3GPP. "Study on channel model for frequencies from 0.5 to $100 \mathrm{GHz}, "$ TR 38.901 v14.3.0, Dec. 2017. [Online]. https://portal.3gpp.org/desktopmodules/Specifications/SpecificationDetails.aspx?specificationId=3173

[190] mmMAGIC. (May, 2017). "Measurement Results and Final mmMAGIC Channel Models," H2020-ICT671650-mmMAGIC/D2.2 v2.0. [Online]. https://5g-mmmagic.eu/results/

[191] D. Tse and P. Viswanath, Fundamentals of Wireless Communication. United Kingdom: Cambridge University Press, 2005.

[192] A.F. Molisch, Wireless Communications. West Sussex, England: John Wiley \& Sons Ltd, 2005.

[193] T. S. Rappaport, Wireless Communications: Principles and Practice.: Prentice-Hall, Inc., 2002.

[194] T.S. Rappaport, G.R. MacCartey, M.K. Samimi, and S. Sun, "Wideband millimeter-wave propagation measurements and channel models for feature wireless communication system design (invited paper)," IEEE Transactions on Communications, vol. 63, no. 9, pp. 3029-3056, Sep. 2015.

[195] S. Sun et al., "Path Loss, Shadow Fading, and Line-Of-Sight Probability Models for 5G Urban MacroCellular Scenarios," in 2015 IEEE Globecom Workshops, San Diego, CA, USA, 2015, pp. 1-7.

[196] ITU-R Rec. P.1238-9, "Propagation data and prediction methods for the planning of indoor radiocommunication systems and radio local area networks in the frequency range $300 \mathrm{MHz}$ to $100 \mathrm{GHz}$," ITU Radiocommunication Bureau, Geneva, Switzerland, 2017.

[197] 3GPP TR 36.873, "Study on 3D channel model for LTE," v12.4.0, March 2017.

[198] 3GPP. "Study on 3D channel model for LTE," TR 36.873 v12.7.0. Dec. 2017. [Online]. https://portal.3gpp.org/desktopmodules/Specifications/SpecificationDetails.aspx?specificationId $=2574$

[199] Fraunhofer Institute for Telecommunications. (2017, January) [Online]. quadriga-channel-model.de/

[200] E. Biglieri et al., MIMO Wireless Communication.: Cambridge University Press, 2007.

[201] J.R. Hampton, Introduction to MIMO Communications.: Cambridge University Press, 2014.

[202] T. Brown, E. De Carvalho, and P. Kyritsi, Practical Guide to MIMO Radio Channel: with MATLAB Examples.: Wiley, 2012.

[203] R. Singh Kshetrimayum, Fundamentals of MIMO Wireless Communications.: Cambridge University Press, 2017.

[204] Geónica. (2017, Feb) [Online]. http://english.geonica.com/docs/9735\%200012\%20DataRain-4000.pdf

[205] R. Gunn and D. Kinzer, "The Terminal Velocity of Fall for Water Droplets in Stagnant Air," Journal of Atmospheric Sciencies, vol. 6, pp. 243-248, August 1949.

[206] P. Garcia-del-Pino, J.M. Garcia-Rubia, J.M. Riera, and A. Benarroch, "Slant-Path Propagation Experiment at Ka-band in Madrid," in The Second European Conference on Antennas and Propagation EuCAP 200\%, Edinburgh, 2007, pp. 1-5.

[207] O. Koudelka, "Q/V-band communications and propagation experiments using ALPHASAT," Acta 
Astronautica, vol. 69, no. 11-12, pp. 1029-1037, 2011.

[208] M. Ruggieri, C. Riva, M. De Sanctis, and T. Rossi, "Alphasat TDP\#5 mission: Towards future EHF satellite communications," in 2012 IEEE First AESS European Conference on Satellite Telecommunications (ESTEL), Rome, Italy , 2012, pp. 1-6.

[209] J.M. Riera, G.A. Siles, P. Garcia-del-Pino, and A. Benarroch, "Alphasat Propagation Experiment in Madrid: Processing of the First Year of Measurements," in The 10th European Conference on Antennas and Propagation (EuCAP 2016), Davos, Switzerland, 2016.

[210] G.A. Siles, J.M Riera, and P. Garcia-del-Pino, "An Application of IGS Zenith Tropospheric Delay Data to Propagation Studies: Validation of Radiometric Atmospheric Attenuation," IEEE Transactions on Antennas and Propagation, vol. 64, no. 1, pp. 262-270, January 2016.

[211] N5173B ENG-X-513. Keysight Technologies. [Online]. http://literature.cdn.keysight.com/litweb/pdf/5991-3132EN.pdf?id=2408626

[212] MAX4M260400. Miteq. [Online]. https://nardamiteq.com/viewmodel.php?model=MAX4M260400

[213] QOM-SL-26-40-K-SG-R. Steatite. [Online]. http://www.steatite-antennas.co.uk/wpcontent/uploads/2017/09/QOM-SL-26-40-K-SG-R.pdf

[214] QSH-SL-26-40-K-20. Steatite. [Online]. http://www.steatite-antennas.co.uk/wpcontent/uploads/2017/09/QSH-SL-26.5-40-K-20.pdf

[215] USB U7227F-8F. Keysigth Technologies. [Online]. https://literature.cdn.keysight.com/litweb/pdf/5991$\underline{4246 \mathrm{EN} . p d f ? \mathrm{id}=2463640}$

[216] M9362AD01. Keysight Technologies. [Online]. https://www.keysight.com/en/pd-1917301-pn-M9362AD01/pxie-microwave-quad-downconverter-10-mhz-to-265-ghz?pm=PL\&nid=$33317.959349 \& \mathrm{cc}=\mathrm{US} \& \mathrm{lc}=\mathrm{eng}$

[217] M9018A. Keysight Technologies. [Online]. https://literature.cdn.keysight.com/litweb/pdf/5990$\underline{6583 \mathrm{EN} \cdot \mathrm{pdf} ? \mathrm{id}=2001178}$

[218] Fairvierw Microwave. (2018, October) [Online]. https://www.fairviewmicrowave.com/images/productPDF/SA40-15.pdf

[219] M9352A. Keysight Technologies. [Online]. https://literature.cdn.keysight.com/litweb/pdf/59909964EN.pdf?id=2164896

[220] DSOS104A. Keysight Technologies. [Online]. https://www.keysight.com/en/pdx-x202068-pnDSOS104A/high-definition-oscilloscope-1-ghz-4-analog-channels?nid=$\underline{32531.1150279 \& \mathrm{cc}=\mathrm{US} \& \mathrm{lc}=\mathrm{eng} \& \mathrm{pm}=\mathrm{OV}}$

[221] ITU-R Study Group 3, "On testing variables used for the selection of prediction methods," ITU-R, Fascicle Document 3M/FAS/1-E, July 2016.

[222] ITU-R Rec. P.581-12, "The concept of "worst month"," ITU Radiocommunication Bureau, Geneva, Switzerland, 1990.

[223] D. Pimienta-del-Valle, J.M. Riera, P. Garcia-del-Pino, and G.A. Siles, "Three-year fade and inter-fade duration statistics from the Q-band Alphasat propagation experiment in Madrid," International Journal of Satellite Communication and Networking, 2018; 1-17. https://doi.org/10.1002/sat.1271.

[224] D. Pimienta-del-Valle, J.M. Riera, and P. Garcia-del-Pino, "Ka- and Q/V-Band Inter-Fade Duration Modeling," IEEE Transactions on Antennas and Propagation, vol. 66, no. 12, pp. 7511-7514, 2018.

[225] R.A. Waltz, J.L. Morales, J. Nocedal, and D. Orban, "An interior algorithm for nonlinear optimization that combines line search and trust region steps," Mathematical Programming, vol. 7, no. 3, July 2006.

[226] D. Pimienta-del-Valle, J.M. Riera, and P. Garcia-del-Pino, "Time and Orbital Diversity assessment with Ka- and Q-Band slant-path propagation experiments in Madrid," IEEE Transactions on Antennas and 
Propagation, vol. 67, no. 2, pp. 1193-1201, 2019.

[227] C. Riva et al., "Development, implementation and testing of analysis tools," ESA study 16865/03/NL/EC, doc. Channel_TR2_analysis, December 2003.

[228] P. Garcia-del-Pino, D. Pimienta-del-Valle, A. Benarroch, and J.M. Riera, "Evaluation of recent prediction models using a long-term database of rainfall rate data," in 2017 11th European Conference on Antennas and Propagation (EUCAP), Paris, 2017, pp. 20-24.

[229] ESA, "Development of high order propagation models for multimedia satellite communications systems," Final Report of ESA/ ESTEC Contract N 40000107135/12/NL/LVH 2016. 